\title{
Ab initio molecular dynamics simulations of molecular scattering from metal(111) surfaces
}

\author{
Dissertation \\ zur Erlangung des mathematisch-naturwissenschaftlichen Doktorgrades \\ "Doktor rerum naturalium" \\ der Georg-August-Universität Göttingen \\ im Promotionsprogramm Chemie \\ der Georg-August University School of Science (GAUSS)
}

vorgelegt von

Jan Altschäffel

aus Göttingen

Göttingen, 2021 
Betreuungsausschuss

Prof. Dr. Alec M. Wodtke, Institut für Physikalische Chemie, Georg-August-Universität Göttingen

Prof. Dr. Dirk Schwarzer, Max-Planck-Institut für biophysikalische Chemie

Mitglieder der Prüfungskommission

Referent: Prof. Dr. Alec M. Wodtke, Institut für Physikalische Chemie, Georg-AugustUniversität Göttingen

Koreferent: Prof. Dr. Dirk Schwarzer, Max-Planck-Institut für biophysikalische Chemie

Weitere Mitglieder der Prüfungskommission

Prof. Dr. Jörg Behler, Institut für Physikalische Chemie, Georg-August-Universität Göttingen

Prof. Dr. Peter E. Blöchl, Institut für Theoretische Physik, Technische Universität Clausthal

Prof. Dr. Burkhard Geil, Institut für Physikalische Chemie, Georg-August-Universität Göttingen

Prof. Dr. Claus Ropers, IV. Physikalisches Institut, Georg-August-Universität Göttingen Tag der mündlichen Prüfung: 27.05.2021 

"Chemistry means the difference between poverty and starvation and the abundant life."

Robert Brent, The Golden Book of Chemistry [1]

"Eine neue wissenschaftliche Wahrheit pflegt sich nicht in der Weise durchzusetzen, daß ihre Gegner überzeugt werden und sich als belehrt erklären, sondern vielmehr dadurch, daß ihre Gegner allmählich aussterben und daß die heranwachsende Generation von vornherein mit der Wahrheit vertraut gemacht ist."

Max Planck, Wissenschaftliche Selbstbiographie [2]

"Ik ben nu totaal gefascineerd door alles wat er met die processen gebeurt en die investeringsagenda! Ik bedoel ik zou er zo kunnen solliciteren. Ja, ja, ja, oprecht ja. Ja, omdat het, omdat het, prachtig is! Maar dat moeten we natuurlijk, dat moeten we vasthouden en uitdragen."

Eric Wiebes over the Dutch tax office (belastingdienst) in a hearing at the Dutch Parliament (Tweede Kamer) on February 2, 2017. 


\begin{abstract}
In this thesis, I report on ab initio molecular dynamics (AIMD) simulations of scattering experiments of $\mathrm{CO}$ and $\mathrm{NO}$ from $\mathrm{Au}(111)$ and $\mathrm{Ag}(111)$ surfaces and provide minimum energy pathway (MEP) calculations of the dissociation reactions of hydrogen halides and NO on different metal(111) surfaces. Furthermore, I did calculations of the transition dipole moment to investigate the electronic interaction of $\mathrm{CO}$ on different surfaces and calculated the electronic ground state energy for CO at different surfaces and $\mathrm{NO} / \mathrm{Au}(111)$. Finally, I computed the phonon spectra of (Ag-covered) Au(111) and $\mathrm{Ag}(111)$ surfaces to look into their mechanical properties, because they can influence the scattering behavior.

A comparison between experimental results and the adiabatic simulations for CO in low vibrational states shows a nearly quantitative agreement, and thus non-adiabatic effects, like electron-hole pair (ehp) excitation, can be neglected. The disagreement between the adiabatic simulations and the experiment suggests a more dominant role of non-adiabatic effects in the scattering dynamics of highly vibrationally excited NO and CO from metal surfaces.

The computed phonon spectra for $\mathrm{Au}(111), \mathrm{Ag}(111)$ and Ag-covered $\mathrm{Au}(111)$ surfaces suggest a purely mechanical energy transfer for the translational energy of $\operatorname{CO}(v=2)$ to the surface phonons which is in agreement with the results of the computed AIMD simulations.

The transition state (TS) configurations for all dissociation reactions show an elongated molecular internuclear distance, and therefore we can assume that the dissociation reactions are promoted via vibrational excitation according to Polanyi's rules.

The obtained density functional theory (DFT) data for $\mathrm{NO} / \mathrm{Au}(111)$ can be fitted to obtain a diabatic potential with a neutral and an anionic state, where $\mathrm{NO}^{-}$is formed to enable molecular dynamics (MD) simulations in the framework of the independent electron surface hopping (IESH) approach.
\end{abstract}




\section{Acknowledgments}

First of all, I would like to thank Prof. Dr. Alec M. Wodtke for giving me the opportunity to work on a very interesting project and his advice over the last years. My deep gratitude goes to Dr. Alexander Kandratsenka for his help regarding all the problems I had especially over the last year, and his patient explanations of all questions I had. Moreover, I thank him for showing me the light at the end of the tunnel when I did not see it. I thank Prof. Dr. Dirk Schwarzer for his fruitful input during the meetings of my advisory committee.

I thank all the members of the Wodtke group for their warm welcome, the excellent atmosphere, and the (often funny) discussion after lunch.

For their never ending support of my efforts and their belief in me I am grateful to my whole family.

My special thanks go to Georgina A. Harrison, Inge Dreger, Dr. Theresa Schweizer, Alexander Kellmann, and Nils Hertl for their efforts in the proof-reading and their comments on the text.

Finally, I thank Nele Niesse for exercises to keep myself fit during the writing phase and David Nolte for his lessons in music and our encouraging talks. 


\section{Contents}

$\begin{array}{ll}\text { Acronyms } & \text { XV }\end{array}$

1 Introduction 1

2 Theoretical background 5

2.1 Born-Oppenheimer Approximation and potential energy surface $\quad 5$

$\begin{array}{ll}2.2 \text { Density functional theory } & 10\end{array}$

$\begin{array}{ll}\text { 2.2.1 Exchange and Correlation } & 13\end{array}$

2.2.2 Approaches to the exchange-correlation functional 15

2.3 Electrons in periodic systems and treatment of electronic occupation of $\begin{array}{ll}\text { metallic bands } & 17\end{array}$

$\begin{array}{ll}2.4 \text { Ab initio molecular dynamics } & 19\end{array}$

3 Methodology 22

3.1 Vienna ab initio simulation package 22

3.1.1 INCAR-file 22

3.1.2 KPOINTS-file 23

$\begin{array}{ll}\text { 3.1.3 POSCAR-file } & 24\end{array}$

3.1.4 POTCAR-file 25

3.2 Fritz Haber Institute ab initio molecular simulations 26

$\begin{array}{ll}3.2 .1 \text { geometry.in-file } & 27\end{array}$

$\begin{array}{ll}3.2 .2 \text { control.in-file } & 28\end{array}$

3.2.3 MEP calculations in FHI-aims $\quad 30$

$\begin{array}{ll}3.3 \text { Phonopy } & 30\end{array}$

$\begin{array}{ll}3.4 \text { Bader analysis } & 32\end{array}$

3.5 Finding minimum energy pathways 33

4 Build up the system and analyze the results 35

4.1 Building up the System 35

$\begin{array}{ll}\text { 4.1.1 fcc-lattice geometry } & 35\end{array}$ 
4.1.2 Preparing the simulation cell 38

4.1.2.1 Equilibrium lattice constant of the $f c c$ metal 38

$\begin{array}{ll}\text { 4.1.2.2 (111) surface } & 39\end{array}$

4.2 Procedure to prepare AIMD simulations 41

4.2.1 Preparation of the initial state of diatomic molecules 41

4.3 Analysis of the energy transfer from AIMD trajectories 45

4.4 Analysis of the translational, rotational and vibrational energy transfer $\begin{array}{ll}\text { from trajectories } & 47\end{array}$

4.5 Transition dipole moment of carbon monoxide on surfaces 52

5 Phonon spectra for $\mathrm{Au}(111), \mathbf{A g}(111)$ and $\mathrm{Ag}$-covered $\mathrm{Au}(111)$ surfaces 56

5.1 Introduction $\quad 56$

$\begin{array}{ll}5.2 \text { Results } & 58\end{array}$

$\begin{array}{ll}5.3 \text { Conclusions } & 66\end{array}$

6 MEPs for dissociation and oxidation of diatomics on metal surfaces 67

$\begin{array}{ll}6.1 \text { Introduction } & 67\end{array}$

6.2 Hydrogen halides on $\mathrm{Au}(111)$ and $\mathrm{Ag}(111) \quad 67$

6.3 NO on $\mathrm{Au}(111), \mathrm{Ag}(111)$ and $\mathrm{Cu}(111) \quad 75$

6.4 CO oxidation on $\mathrm{Pt}(111) \quad 80$

$\begin{array}{ll}6.5 \text { Conclusions } & 88\end{array}$

7 Interaction of diatomic molecules with (111) metal surfaces 90

$\begin{array}{ll}7.1 \text { Introduction } & 90\end{array}$

$\begin{array}{ll}7.2 \mathrm{CO} / \mathrm{Au}(111) & 90\end{array}$

7.3 $\mathrm{CO} / \mathrm{Ag}(111)$ and $\mathrm{CO}$ at Ag-covered $\mathrm{Au}(111) \quad 93$

$\begin{array}{ll}7.4 \mathrm{NO} \text { at } \mathrm{Au}(111) & 95\end{array}$

7.4.1 Convergence tests for $\mathrm{NO} / \mathrm{Au}(111) \quad 98$

7.4.2 Energy grid for $\mathrm{NO} / \mathrm{Au}(111) \quad 104$

$\begin{array}{ll}\text { 7.4.3 NO molecule in vacuum } & 108\end{array}$

7.4.4 Investigating the Bader analysis using different input files $\quad 109$

$\begin{array}{ll}7.5 \text { Conclusions } & 112\end{array}$

8 AIMD simulations for scattering of CO and NO from (111) metal surfaces 114

$\begin{array}{lll}8.1 \text { Introduction } & 114\end{array}$

$\begin{array}{ll}8.2 \mathrm{CO}(\nu=0) \text { from } \mathrm{Au}(111) & 115\end{array}$

8.3 $\mathrm{CO}(v=2)$ from $\mathrm{Au}(111), \mathrm{Ag}(111)$ and Ag-covered $\mathrm{Au}(111) \quad 119$

$8.4 \mathrm{CO}(v=17,22)$ from $\mathrm{Au}(111)$ and $\mathrm{Ag}(111) \quad 124$

$\begin{array}{ll}\text { 8.4.1 Simulations using VASP } & 124\end{array}$ 
8.4.2 Simulations with FHI-aims $\quad 127$

8.4.3 Analysis of the forces 136

$8.5 \mathrm{NO}(\nu=16)$ from $\mathrm{Au}(111)$ and $\mathrm{Ag}(111) \quad 138$

$\begin{array}{ll}8.6 \text { Conclusions } & 140\end{array}$

9 Transition dipole moments of carbon monoxide on different surfaces $\quad 143$

$\begin{array}{ll}9.1 \text { Introduction } & 143\end{array}$

$\begin{array}{ll}9.2 \mathrm{CO} \text { on } \mathrm{Au}(111) & 143\end{array}$

9.3 CO on Ag(111) and Ag-covered $\mathrm{Au}(111) \quad 147$

$\begin{array}{ll}9.4 \mathrm{CO} \text { on } \mathrm{NaCl}(100) & 151\end{array}$

$\begin{array}{ll}9.5 \text { Conclusions } & 153\end{array}$

10 Discussion 155

$\begin{array}{ll}\text { Bibliography } & \text { XVII }\end{array}$

A Appendix $\quad$ XXV

A.1 Example input files for VASP $\quad$ XXV

A.1.1 Input files for an AIMD simulation of $\mathrm{CO}(v=17)$ in vacuum $\quad$ XXV

A.1.2 Input files for an energy grid calculation for $\mathrm{NO} / \mathrm{Au}(111) \quad \mathrm{XXV}$

A.2 Example input files for FHI-aims $\quad$ XXX

A.2.1 Input files for an AIMD simulation of $\mathrm{CO}(\nu=17) / \operatorname{Ag}(111) \quad \mathrm{XXX}$

A.2.2 Input files for a MEP calculation XXX

A.3 geometry . in-files for $\mathrm{CO}$ on $\mathrm{NaCl}(100) \quad$ XXXVII 


\section{List of Figures}

Fig. 1 Exemplary INCAR-file of an Au bulk calculation. 23

Fig. 2 Exemplary KPOINTS-file for an Au bulk calculation. 24

Fig. 3 Exemplary POSCAR-file for an Au bulk calculation. 25

Fig. 4 Exemplary geometry . in-file for a trajectory of $\operatorname{CO}(v=17)$ in vacuum. 28

Fig. 5 Exemplary control. in-file for a trajectory of $\mathrm{CO}(\nu=17)$ in vacuum (without the basis set settings).

Fig. 6 A crystal in its $f c c$ metal structure 36

Fig. 7 Cohesive energy of an Au bulk vs. the lattice constant. 38

$\begin{array}{lll}\text { Fig. } 8 \text { A } \mathrm{p}(3 \times 3) \text { and simulation cell. } & 40\end{array}$

Fig. 9 Energy exchange along an exemplary trajectory. 48

$\begin{array}{lll}\text { Fig. } 10 \text { Contributions to the rotational energy. } & 49\end{array}$

Fig. 11 Potential energy of a diatomic molecule as function of the

$\begin{array}{ll}\text { internuclear distance. } & 50\end{array}$

Fig. 12 Experimental mean final translational energy vs. the Ag-film thickness. 57

Fig. 13 Phonon spectra for a chain and two bulk models. 59

$\begin{array}{lll}\text { Fig. } 14 & \text { Phonon spectra for two bulk models. } & 60\end{array}$

Fig. 15 Phonon spectra for different models of an Ag(111) surface. 60

Fig. 16 Phonon spectra for an Ag bulk and Ag surface models. 63

Fig. 17 Phonon spectra for different (111) surfaces. 64

Fig. 18 Highest frequency of the phonon spectrum vs. the number of silver layers.

Fig. 19 Initial and final states for the MEP calculations of $\mathrm{HCl}$ on $\mathrm{Au}(111)$

and $\mathrm{Ag}(111)$. 68

Fig. 20 MEP configurations for the hydrogen halides on $\mathrm{Au}(111)$ and $\mathrm{Ag}(111) .71$

Fig. 21 TS configurations for the hydrogen halides on Au and Ag(111). 73

Fig. 22 The MEP for the dissociation of $\mathrm{HCl}$ on $\mathrm{Au}(111)$. 74

Fig. 23 MEPs for the dissociation of $\mathrm{HCl}$ and $\mathrm{HF}$ on $\mathrm{Au}(111)$ and $\mathrm{Ag}(111) . \quad 74$

Fig. 24 Initial and final states for the MEPs of NO on noble metal(111) surfaces. 77 
$\begin{array}{lll}\text { Fig. } 25 & \text { TS configurations for NO on noble metal(111) surfaces. } & 78\end{array}$

Fig. 26 MEPs for NO dissociation on the noble metal surfaces. 79

Fig. 27 Initial and final configurations for the CO oxidation on $\operatorname{Pt}(111)$.

Fig. 28 TS configurations for CO oxidation on Pt(111). 83

Fig. 29 MEPs of the CO oxidation on Pt(111). 83

Fig. 30 MEPs of the O diffusion on Pt(111). 83

Fig. 31 Two configurations of the MEP of the CO oxidation in the $p(3 \times 3)$ cell. 85

Fig. 32 Final translational energy distribution of the formed $\mathrm{CO}_{2}$. 86

Fig. 33 Scheme of the MEP including the TS configuration. 87

Fig. $34 \mathrm{CO} / \mathrm{Au}(111)$ interaction energy as function of the CO COM distance to the surface. $\quad 92$

Fig. 35 Interaction energy of CO and different metal(111) surfaces. 94

Fig. $36 \mathrm{NO} / \mathrm{Au}(111)$ interaction energy as function of the NO COM distance to the surface. $\quad 96$

Fig. 37 Energy and computational time vs. the cut-off energy. 98

Fig. 38 Energy and computational time vs. the square root of $\boldsymbol{k}$-points. 99

Fig. $39 \mathrm{NO} / \mathrm{Au}(111)$ interaction energy for different $\boldsymbol{k}$-point grids. $\quad 101$

$\begin{array}{ll}\text { Fig. } 40 \text { Different energies vs. } \sigma \text { for } \mathrm{NO} / \mathrm{Au}(111) \text {. } & 102\end{array}$

Fig. 41 Magnetic moment and computational time vs. $\sigma . \quad 103$

Fig. 42 Energy vs. $\sigma$ for different configurations of $\mathrm{NO} / \mathrm{Au}(111) . \quad 105$

$\begin{array}{ll}\text { Fig. } 43 \text { DFT energies and Bader charges for } \mathrm{NO} / \mathrm{Au}(111) . & 107\end{array}$

Fig. 44 Translational energy distribution for $\mathrm{CO}(\nu=0)$ scattered from $\mathrm{Au}(111) .116$

Fig. 45 Coupling between final rotational and translational energies for

$\mathrm{CO}(\nu=0)$ scattered from $\mathrm{Au}(111)$.

Fig. 46 Final translational energy distribution of simulations of $\mathrm{CO}(\nu=0)$ scattered from $\mathrm{Au}(111)$ at $300 \mathrm{~K}$.

Fig. 47 Distribution of the energy transfer to the slab energy $\left(\Delta \mathrm{E}_{\text {slab }}\right)$

distribution of simulations of $\mathrm{CO}(\nu=0)$ scattered from $\mathrm{Au}(111)$ at $300 \mathrm{~K}$.

Fig. 48 Potential energy of the CO molecule as a function of the bond distance and corresponding Morse fit.

Fig. 49 Final translational energy distribution for $\mathrm{CO}(v=2)$ scattered from different surfaces.

Fig. 50 Coupling between different dofs of $\mathrm{CO}(v=2)$ scattered from different metal(111) surfaces.

Fig. 51 A trajectory of $\mathrm{CO}\left(T_{\mathrm{in}}=1 \mathrm{eV}, E_{\mathrm{vib}}=6 \mathrm{eV}\right)$ approaching an $\mathrm{Au}(111)$ surface. 
Fig. 52 A trajectory of $\mathrm{CO}\left(T_{\mathrm{in}}=1 \mathrm{eV}, E_{\mathrm{vib}}=6 \mathrm{eV}\right)$ in vacuum.

Fig. 53 Potential energy differences and final magnetic moments for different spin treatments.

Fig. 54 Trajectory of $\mathrm{CO}(=17)$ in vacuum calculated with FHI-aims.

Fig. 55 Trajectory of $\mathrm{CO}(\nu=17)$ scattered from $\mathrm{Au}(111)$ calculated with FHI-aims.

Fig. 56 Final translational energy distribution for $\mathrm{CO}(v=17 \rightarrow 17)$ scattered from $\mathrm{Au}(111)$ and $\operatorname{Ag}(111)$.

Fig. 57 Final vibrational state distribution for $\operatorname{CO}(v=17)$ scattered from $\mathrm{Au}(111)$ and $\mathrm{Ag}(111)$.

Fig. 58 Final vibrational state distributions for $\mathrm{CO}(\nu=17)$ for different initial orientation of the molecule.

Fig. 59 Final vibrational state distribution (probability density) for

$\mathrm{CO}\left(v_{\text {in }}=22\right)$ scattered from $\mathrm{Ag}(111)$ (top panel) and $\mathrm{Au}(111)$ (bottom).

Fig. 60 Analysis of the forces for a trajectory for $\mathrm{CO}(\nu=17) / \operatorname{Ag}(111)$ at $300 \mathrm{~K} . \quad 137$

Fig. 61 Final vibrational state distribution for $\mathrm{NO}(\nu=16)$ scattered $\mathrm{Au}(111)$ and $\mathrm{Ag}(111)$.

Fig. 62 The so-called Krüger plot for the investigated systems.

Fig. 63 Change in the dipole moment vs. the $\mathrm{CO}$ bond distance for $\mathrm{CO}$ at $\mathrm{Au}(111)$ and in vacuum.

Fig. 64 Change in the dipole moment vs. the $\mathrm{CO}$ bond distance for $\mathrm{CO}$ at metal(111) surfaces and in vacuum.

Fig. A.1 INCAR-file for an AIMD simulation for a CO molecule in vacuum.

Fig. A.2 KPOINTS-file for an AIMD simulation for a CO molecule in vacuum. XXVI

Fig. A.3 POSCAR-file for an AIMD simulation for a CO molecule in vacuum. $\quad$ XXVII

Fig. A.4 INCAR-file for a NO/Au(111) calculation. $\quad$ XXVII

Fig. A.5 KPOINTS-file for for a NO/Au(111) calculation. XXVIII

Fig. A.6 POSCAR-file for a $\mathrm{NO} / \mathrm{Au}(111)$ calculation. $\quad$ XXIX

Fig. A.7 control. in-file without basis set settings as used for exemplary AIMD simulations of $\mathrm{CO}(v=17) / \mathrm{Ag}(111)$.

Fig. A.8 Basis set settings (light) for the $\mathrm{C}$ atom. $\quad$ XXXII

Fig. A.9 Basis set settings (light) for the $\mathrm{O}$ atom. XXXIII

Fig. A.10 geometry. in-file of an exemplary trajectory for $\mathrm{CO}(\nu=17)$

scattered from $\operatorname{Ag}(111)$ XXXIV

Fig. A.11 chain. in-file as used in the MEP calculation of the CO oxidation. XXXV 
Fig. A.12 control . in-file without basis set settings as used in the MEP calculation of the $\mathrm{CO}$ oxidation.

XXXV

Fig. A.13 ini . in-file for the CO oxidation on a $\mathrm{p}(3 \times 3)$ slab with 4 layers.

XXXVI

Fig. A.14 $\mathrm{f}$ in . in-file for the CO oxidation on a $\mathrm{p}(3 \times 3)$ slab with 4 layers.

XXXVI

Fig. A.15 geometry. in-files of the used $\mathrm{c}(2 \times 1)$ cells for $\mathrm{NaCl}(100)$.

XXXVII 


\section{List of Tables}

Tab. 1 initial energies $E_{\text {in }}$, final energies $E_{\mathrm{f}}$ and energy loss $E_{\text {loss }}$ of the different dofs of the molecule, $E_{\mathrm{mol}}, E_{\mathrm{slab}}$ and $E_{\mathrm{tot}}$ of the trajectory from Fig. 9 are represented.

Tab. 2 Structural parameters for the MEP configurations of hydrogen halides on metal(111) surfaces.

Tab. 3 Structural parameters for the initial, final and transition states for the MEP calculations of NO on $\mathrm{Au}(111), \mathrm{Ag}(111)$ and $\mathrm{Cu}(111)$.

Tab. 4 Structural parameters for the MEP configurations of the CO oxidation on $\operatorname{Pt}(111)$.

Tab. 5 Computational details and results for NO in vacuum calculated with VASP and RPBE.

Tab. 6 Bader charges for different ways of analysis.

Tab. 7 Structural parameters of the geometry optimizations for CO on Au(111).144

Tab. 8 Fit parameters for the fit function to describe the dipole moment $\begin{array}{ll}\text { operator for } \mathrm{CO} \text { at } \mathrm{Au}(111) . & 146\end{array}$

Tab. 9 Matrix elements of the dipole moment for CO at Au(111). 147

Tab. 10 Structural parameters for the geometry optimizations for CO on metal(111) surfaces.

Tab. 11 Morse parameters and parameters of the function to describe the dipole moment operator for $\mathrm{CO}$ at Ag-covered $\mathrm{Au}$ (111) surfaces.

Tab. 12 Matrix elements of the dipole moment for CO at Ag-covered Au(111) surfaces.

Tab. 13 Morse parameters and parameters of the function to describe the dipole moment operator for $\mathrm{CO}$ at $\mathrm{NaCl}(100)$.

Tab. 14 Matrix elements of the dipole moment for $\mathrm{CO}$ at $\mathrm{NaCl}(100)$. 


\section{Acronyms}

$\begin{array}{ll}\text { AIMD } & \text { ab initio molecular dynamics } \\ \text { BOA } & \text { Born-Oppenheimer approximation } \\ \text { COM } & \text { center-of-mass } \\ \text { DFT } & \text { density functional theory } \\ \text { dof } & \text { degree(s) of freedom } \\ \text { DOS } & \text { density of states } \\ \text { EF } & \text { electronic friction } \\ \text { ehp } & \text { electron-hole pair } \\ \text { EwoS } & \text { energy without entropy } \\ \text { F } & \text { free energy } \\ \text { fcc } & \text { face-centered cubic } \\ \text { FHI-aims } & \text { Fritz-Haber-Institute } \text { ab initio } \text { molecular simulations } \\ \text { GGA } & \text { generalized-gradient approximation } \\ \text { hcp } & \text { hexagonal-closed packed } \\ \text { IBZ } & \text { inner Brillouin zone } \\ \text { IESH } & \text { independent electron surface hopping } \\ \text { LDA } & \text { local-density approximation } \\ \text { LSD } & \text { local-spin-density approximation } \\ \text { MD } & \text { molecular dynamics } \\ \text { MEP } & \text { minimum energy pathway } \\ \text { MP } & \text { Methfessel-Paxton } \\ \text { NAO } & \text { numeric atom-centered orbital } \\ \text { NEB } & \text { nudged elastic band } \\ \text { PAW } & \text { projector augmented-wave } \\ \text { pbc } & \text { periodic boundary conditions } \\ \text { PBE } & \text { Perdew Burke Ernzerhofer, a GGA functional } \\ \text { PBEsol } & \text { revised Perdew Burke Ernzerhofer for solids, a GGA } \\ \text { PDOS } & \text { functional } \\ \text { PES } & \text { projected density of states } \\ & \text { potential energy surface } \\ \text { ind }\end{array}$


PP pseudo potential

PW91 Perdew and Wang of 1991, a GGA functional

RPBE revised Perdew Burke Ernzerhofer, a GGA functional

scf self-consistent field

SE Schrödinger equation

TS transition state

ueg uniform electron gas

VASP Vienna $A b$ initio Simulation Package

vdW van-der-Waals

VEBE vertical electron binding energy

$\mathrm{XC}$ exchange-correlation 


\section{Introduction}

Reactions of diatomic molecules at metal surfaces play an important role in chemistry, especially in heterogeneous catalysis [3-6]. Since heterogeneous catalysis is very important for industry, a lot of research has been done in this field over the last decades. Research has been done to get on one hand a better understanding in the catalytic processes and on the other hand to find new and more efficient catalysts. A detailed understanding strongly improves the search for catalysts. Thus, if the mechanism of a catalytic reaction is completely known it is possible to modify the catalyst in a selective way so that the reaction can be improved. Decades ago most improvements in catalysts used to speed up a chemical reaction were found by trial and error approaches with different substances or by accident, for example in the case of the ZIEGLER-NATTA catalysts [7]. In the last three decades the understanding of heterogeneous catalysis has grown, entire groups of substances which seem to have a catalytic activity were computationally screened. This screening of potential catalytic candidates has made the search for catalysts more efficient $[8,9]$. However, predictions of new catalysts based on the knowledge of on-going processes are rarely exceptional [10] than are daily routine. Therefore, a more detailed understanding of the processes and mechanisms happening during heterogeneous catalytic reactions is needed.

A key factor within the route understanding molecule-surface reactions is the building of a picture of the energy transfer between the two subsystems. For this purpose molecular beam scattering experiments from surfaces were done over the last decades [11-14]. These quantum-state-resolved experiments show that the translational, rotational and vibrational degree(s) of freedom (dof)s of the molecule couple to the electronic motion of the surface. Especially, the system of NO/Au(111) has been investigated in detail [1524 ] to elucidate the different pathways of the energy transfer between the molecule and the surface. The coupling of the molecular vibration to the surface electrons was found by HUANG et al., in which they compared the scattering of highly vibrationally excited $\mathrm{NO}(v=16)$ from $\mathrm{Au}(111)$ to the scattering from an insulator $\mathrm{LiF}$ [15]. In the first case the molecule loses a lot of its vibrational energy when scattered back from the surface, i.e. the vibrational state is significantly lower than the initial one. In the second case the molecules remain in their initial vibrational state after they are scattered back from the 
insulating surface. Here, the band gap is too large so the possible vibrational energy loss is not large enough to excite electrons from the valence into the conduction band. Scattering experiments of $\mathrm{NO}(v=16)$ from a Cs-coated $\mathrm{Au}(111)$ surface even show the production of free electrons (exo-electrons) accompanied with a large vibrational energy loss of the molecule, due to the lowered work function of the surface compared to the pure gold surface $[23,24]$.

Other evidence for the coupling of molecular vibrational to electronic dof of a metal surface was seen in an investigation of the vibrational lifetime of $\mathrm{CO}$ on $\mathrm{Cu}(100)$ [25]; here the lifetime was nine orders of magnitude shorter than the one found on an insulator, e.g. $\mathrm{NaCl}(100)$ [26]. The coupling of nuclear translational dofs to electronic ones of a metal surface is observed when $\mathrm{H}$ atoms stick on an $\mathrm{Au}(111)$-surface because an adiabatic model does not predict enough energy loss that the atom can stick on the surface [27]. The clear evidence that molecular motion couples to electronic dofs is the observation of chemicurrents, which were first seen when $\mathrm{H}$ atoms adsorb on thin silver films which coats a silicon wafer [28]. Thus, a small current, i.e. electronic motion, is induced by the atomic adsorption. All these examples show how prominent coupling between the dofs of the adsorbate and the electrons and phonons of the surface is. So a model that hopes to describe the energy transfer dynamics correctly, must take these couplings into account. A very accurate model would easily make predictions concerning more efficient catalysts. But here is the problem, because the most important approximation in theoretical chemistry, the Born-Oppenheimer approximation (BOA) [29], does not include the coupling between the electrons of the surface and the nuclear motion of the adsorbate and so it cannot be used as basis for a model describing certain processes at surfaces. As the experimental studies investigating processes of molecules at surfaces have been more and more elaborated, theoretical efforts have been made as well to shed light on these processes.

These theoretical efforts are mostly based on DFT calculations, i.e. calculating the electronic ground state energy as functional of the electron density, which is described in section 2.2. Two codes to perform such calculations in practice are described in chapter 3 . From such calculations we can obtain a potential energy surface (PES) (see section 2.1) and that means an energy landscape depending on the coordinates of all atoms in the system. The valleys and heights of this landscape are the regions where the atoms show attraction and repulsion to each other. To produce a high-dimensional PES a fitting procedure to the calculated DFT data is necessary. One can use the PES to perform classical MD simulations to get insights into the dynamics of the system of interest [30]. A more straightforward way to do this is to do AIMD simulations, as described in section 2.4, which beforehand do not need a PES. These simulations are 
an excellent tool to describe the dynamics of atoms and molecules at metal surfaces. This was shown by NATTINO and co-workers for the dissociative adsorption of $\mathrm{D}_{2}$ on $\mathrm{Cu}(111)$ who found a good agreement between their simulations and the corresponding experimental results $[31,32]$. This is also true for the dissociation of methane on metal surfaces where AIMD simulations were able to describe important characteristics of scattering experiments [33, 34].

If one is interested in certain features of a reaction, like the barrier height, one can investigate only a special region on the PES. For this purpose, MEP calculations can be carried out, as described section 3.5. In this thesis, the reactions of diatomic molecules on different metal(111) surfaces were investigated by MEP calculations, which are presented in chapter 6. Since experimental [35] and theoretical studies [36] on the dissociative adsorption of $\mathrm{HCl}$ on $\mathrm{Au}(111)$ reveal differences in the reaction probability, calculations with a different exchange-correlation (XC) functional from the one used in [36] may lead to a better agreement between the experiment and theory. Furthermore, the MEP calculations can give insights into the question if vibrationally excited molecules can promote the dissociation reaction, which can be concluded from the configuration of the TS following Polanyi's rules [37]. The CO oxidation on Pt(111) was studied by MEP and AIMD calculations, which can be found in section 6.4 to support experimental observations on this system [38] and to back the assignment of these observations to elementary steps on the different regions on the surface, i.e. flat and stepped regions. The investigation of STEINSIEK et al. [39] shows strong difference in the translational inelasticity of scattered CO and NO from pure and Ag-coated Au(111) surfaces. As the reason for these observations was not clear different aspects which may influence them were investigated here. The investigations comprise the electronic interaction between $\mathrm{CO}$ and the metal surfaces as described in chapter 9, the energetics of the systems as shown in chapter 7 and the mechanical properties of the different surfaces as presented in chapter 5. Additionally, AIMD simulations were performed to shed light on the energy transfer between the impinging molecule and metal surface, which can be found in section 8.3.

More recently, scattering experiments for CO in low [39-41] and high [42, 43] vibrational states and different metal (111)-surfaces were carried out. These experiments reveal that the vibrational relaxation of $\mathrm{CO}(v=2)$ is quite different from that of $\mathrm{CO}(v=17)$. In the former case only $2 \%$ of the scattered molecules are in a lower vibrational state, whereas in the latter case about $30 \%$ of the molecules come back in a lower vibrational state. Thus, here the vibrational relaxation is not so prominent as in the case of $\mathrm{NO} / \mathrm{Au}(111)$. This difference seems to be due to a better stabilization of $\mathrm{NO}^{-}$at the $\mathrm{Au}(111)$ surface than that of $\mathrm{CO}^{-}$as the WAGNER et al. stated in [42]. This suggests a transient anion 
formation in front of the surface and the excitation of ehp during the scattering event. Hence, non-adiabatic effects play a role in these scattering events. To get further evidence for the occurrence of these effects, adiabatic simulations on a single PES, i.e. AIMD simulations, of the scattering experiments were performed in this thesis (see chapter 8). How these simulations are prepared and analyzed is described in chapter 4. By comparing the experimental and the simulated results one can see how strong the influence of non-adiabatic effects in the energy transfer during the scattering could be. If the agreement between the results is good for a system we can neglect the nonadiabatic effects here, because they cannot be described by the simulations, whereas large discrepancies between simulations and experimental observations would be a clear evidence for the involvement of non-adiabatic effects in the energy transfer of molecule and surface.

The system $\mathrm{NO} / \mathrm{Au}(111)$ has been investigated in detail in molecule-surface scattering experiments as written above. The molecule was prepared in low and high vibrational states, and vibrational excitation and relaxation was investigated [15, 19]. Not only the initial vibrational state of the molecule was varied, but also its initial orientation was controlled by electric fields and the Stark effect [21,44]. To understand the experimental observations of $\mathrm{NO} / \mathrm{Au}(111)$, the IESH approach $[45,46]$ was employed to simulate the experiments and was successful in describing some experimental results but fails to describe them completely [47]. It seems to be likely that the reason for this failure is due to a wrong diabatic potential on which the simulations are based [48]. More recently, YIN et al. constructed a new ground state PES using the neural network approach [49] and performed adiabatic simulations, which showed agreement with certain aspects of the experiments [50]. This supports the assumption that the failure of the diabatic potential is due to the fitting procedure used to obtain it and not to the input data based on DFT calculations. Furthermore, the NO/Au(111) system was also investigated via an approach based on electronic friction (EF) (this means that the energy transfer to the electronic dofs happens by friction between the nuclei with the electron density in the system) by MONTURET and SAALFRANK [51].

Therefore, a new potential has to be constructed and thereafter IESH simulations should be carried out, again, to see if a stronger agreement between the experiment and simulations could be reached. In this thesis calculations for the system $\mathrm{NO} / \mathrm{Au}(111)$ have be performed to produce DFT data, which is given in section 7.4, and these make the first step on the route to construct a new diabatic potential, which can then be used to perform simulations on this system. 


\section{Theoretical background}

This chapter covers some theoretical aspects which may be helpful to understand the proceedings in the following chapters. I will begin with the basics of the BOA and PES. The second section addresses how to calculate the electronic energy of a many-body system via DFT. After that I explain how electrons in periodic systems are represented and what practical consequences arise from that and how they are solved in DFT codes. In the last section I briefly explain the term MD simulation and its special variant AIMD. The latter describes the nuclear motion on a single PES obtained by solving the electronic Schrödinger equation (SE), whereas the former needs a PES obtained beforehand. The text in the first section is mainly based on the books by Marx and Hutter ([52]) and Baer ([53]). The DFT section is based on the text book by Jensen ([54]) and the one by Koch and Holthausen ([55]). For the AIMD section, I also used the book by Frenkel and Smit ([56]).

\subsection{Born-Oppenheimer Approximation and potential energy surface}

As the concept of the PES derives directly from the BOA, it is appropriate to explain these jointly.

To describe the time evolution of a nonrelativistic system which contains $N$ electrons and $M$ nuclei the time-dependent SE

$$
i \hbar \frac{\partial \Psi(\boldsymbol{r}, \boldsymbol{R}, t)}{\partial t}=\widehat{H} \Psi(\boldsymbol{r}, \boldsymbol{R}, t) .
$$

is used. Here, $\Psi(\boldsymbol{r}, \boldsymbol{R}, t)$ is the wave function depending on time $t$ and the set of all electronic $\boldsymbol{r}=\left(\boldsymbol{r}_{1}, \ldots, \boldsymbol{r}_{N}\right)$ and nuclear coordinates $\boldsymbol{R}=\left(\boldsymbol{R}_{1}, \ldots, \boldsymbol{R}_{M}\right)$. In principle all electronic coordinates also contain a spin component which is neglected here.

The Hamiltonian of (1) is explicitly given by

$$
\widehat{H}=\widehat{T}_{\mathrm{n}}+\widehat{T}_{\mathrm{e}}+\widehat{V}_{\mathrm{nn}}(\boldsymbol{R})+\widehat{V}_{\mathrm{ee}}(\boldsymbol{r})+\widehat{V}_{\mathrm{ne}}(\boldsymbol{r}, \boldsymbol{R}),
$$


where

$$
\widehat{T}_{\mathrm{n}}=-\sum_{\alpha=1}^{M} \frac{\hbar^{2}}{2 M_{\alpha}} \nabla_{\boldsymbol{R}}^{2}
$$

is the kinetic energy operator of the nuclei, and

$$
\widehat{T}_{\mathrm{e}}=-\frac{\hbar^{2}}{2 m_{e}} \sum_{i=1}^{N} \nabla_{\boldsymbol{r}}^{2}
$$

is its electronic counterpart.

$$
\widehat{V}_{\mathrm{nn}}(\boldsymbol{R})=\frac{1}{2} \sum_{\alpha=1}^{M} \sum_{\beta \neq \alpha}^{M} \frac{Z_{\alpha} Z_{\beta} e^{2}}{4 \pi \epsilon_{0}\left|\boldsymbol{R}_{\alpha}-\boldsymbol{R}_{\beta}\right|}
$$

represents the nuclear-nuclear repulsion,

$$
\widehat{V}_{\mathrm{ee}}(\boldsymbol{r})=\frac{1}{2} \sum_{i=1}^{N} \sum_{j \neq i}^{N} \frac{e^{2}}{4 \pi \epsilon_{0}\left|\boldsymbol{r}_{i}-\boldsymbol{r}_{j}\right|}
$$

is the electron-electron repulsion and

$$
\widehat{V}_{\mathrm{ne}}(\boldsymbol{r}, \boldsymbol{R})=-\sum_{i=1}^{N} \sum_{\alpha=1}^{M} \frac{Z_{\alpha} e^{2}}{4 \pi \epsilon_{0}\left|\boldsymbol{r}_{i}-\boldsymbol{R}_{\alpha}\right|}
$$

is the nuclear-electron attraction.

In the equations from (3) to (7) Greek indices run over the nuclei and Latin ones run over the electrons. So $M_{\alpha}$ denotes the mass of the nuclei $\alpha$ and $Z_{\alpha}$ its atomic number. $m_{\mathrm{e}}, e$ and $\epsilon_{0}$ are the electronic mass, the elementary charge and the vacuum permittivity.

Here, $\widehat{H}$ does not depend on the time $t$ explicitly and thus a product ansatz is valid to separate wave function in an time-dependent and time-independent part. This gives the time-independent SE

$$
\widehat{H} \varphi(\boldsymbol{r}, \boldsymbol{R})=E(\boldsymbol{r}, \boldsymbol{R}) \varphi(\boldsymbol{r}, \boldsymbol{R}),
$$

where $\varphi(\boldsymbol{r}, \boldsymbol{R})$ represents the time-independent part of the wave function and $E(\boldsymbol{r}, \boldsymbol{R})$ the total energy.

One can summarize all terms which depend on the electronic degrees of freedom in the electronic Hamiltonian $\widehat{H}_{\mathrm{e}}$ :

$$
\widehat{H}=\widehat{T}_{\mathrm{n}}+\widehat{H}_{\mathrm{e}}
$$

The electronic Hamiltonian $\widehat{H}_{\mathrm{e}}$ also depends on the nuclear coordinates due to the nuclear-electron $\widehat{V}_{\text {ne }}(\boldsymbol{r}, \boldsymbol{R})$ and the nuclear-nuclear $\widehat{V}_{\mathrm{nn}}(\boldsymbol{R})$ interactions. The latter is 
just a constant for each nuclear configuration $\boldsymbol{R}$ and is added to $\widehat{H}_{\mathrm{e}}$ for convenience. The electronic eigenfunctions $\psi_{\ell}(\boldsymbol{r} ; \boldsymbol{R})$, which parametrically depend on $\boldsymbol{R}$ indicated by the semicolon, and eigenvalues $E_{\ell}(\boldsymbol{R})$ are determined from the electronic SE:

$$
\widehat{H}_{\mathrm{e}} \psi_{\ell}(\boldsymbol{r}, \boldsymbol{R})=E_{\mathrm{e}, \ell}(\boldsymbol{R}) \psi_{\ell}(\boldsymbol{r} ; \boldsymbol{R}) .
$$

The eigenvalues as well as $\widehat{H}_{\mathrm{e}}$ can be seen as PES on which the nuclei are moving, and it changes with each change in the electronic configuration described by the $\psi_{\ell}(\boldsymbol{r} ; \boldsymbol{R}) \mathrm{s}$. The electronic wave functions are chosen to be orthonormal, i.e.,

$$
\left\langle\psi_{\ell} \mid \psi_{k}\right\rangle= \begin{cases}1, & \text { if } k=\ell \\ 0, & \text { if } k \neq \ell\end{cases}
$$

where the bra-ket notation is used to indicate integration over all electronic coordinates.

Now we have the tool set to describe the total energy of the system, via solving the time-independent SE, but in practice the wave function in Eq. (8) is far too complicated to solve. Hence, we need another ansatz for the wave function to decouple the two sets of variables $\boldsymbol{R}$ and $\boldsymbol{r}$.

One of the most popular ways to do this to express the wave function in terms of the electronic eigenstates:

$$
\varphi(\boldsymbol{r}, \boldsymbol{R})=\sum_{\ell} \chi_{\ell}(\boldsymbol{R}) \psi_{\ell}(\boldsymbol{r} ; \boldsymbol{R}),
$$

where $\chi_{\ell}(\boldsymbol{R})$ is the wave function for the nuclei in the electronic state $\ell$. The eigenfunctions $\psi_{\ell}(\boldsymbol{r} ; \boldsymbol{R})$ form a complete set. The first to do this expansion, which is an exact description and not an approximation, were Born and Huang [29, 57].

Inserting (12) as ansatz for the wave function into (8) and multiplying from the left with an electronic state $\left\langle\psi_{k}\right|$, where $k=1, \ldots, N$, one obtains

$$
\begin{aligned}
\left\langle\psi_{k}|\widehat{H}| \varphi\right\rangle & =\left\langle\psi_{k}\left|\left(\widehat{H}_{\mathrm{e}}+\widehat{T}_{\mathrm{n}}\right)\right| \varphi\right\rangle \\
& =\sum_{\ell}\left[E_{\mathrm{e}, \ell}(\boldsymbol{R})\left\langle\psi_{k} \mid \psi_{\ell}\right\rangle+\left\langle\psi_{k}\left|\widehat{T}_{\mathrm{n}}\right| \psi_{\ell}\right\rangle\right] \chi_{\ell}(\boldsymbol{R}) \\
& =\left(E_{\mathrm{e}, k}(\boldsymbol{R})+\widehat{T}_{\mathrm{n}}\right) \chi_{k}(\boldsymbol{R}) \\
& +\sum_{\alpha=1}^{M} \frac{\hbar^{2}}{2 M_{\alpha}} \sum_{\ell}\left[\left\langle\psi_{k}\left|\nabla_{\alpha}\right| \psi_{\ell}\right\rangle \nabla_{\alpha}+\left\langle\psi_{k}\left|\nabla_{\alpha}^{2}\right| \psi_{\ell}\right\rangle\right] \chi_{\ell}(\boldsymbol{R}) .
\end{aligned}
$$


Thus, the total energy of the system

$$
\begin{aligned}
E(\boldsymbol{R}) & =\sum_{k}\left[E_{\mathrm{ad}, k}(\boldsymbol{R})+\sum_{\alpha=1}^{M} \frac{\hbar^{2}}{2 M_{\alpha}} \sum_{\ell \neq k}\left(\boldsymbol{d}_{\alpha, k \ell} \nabla_{\alpha}+D_{\alpha, k \ell}\right)\right] \\
E_{\mathrm{ad}, k}(\boldsymbol{R}) & =E_{\mathrm{BO}, k}(\boldsymbol{R})+\sum_{\alpha=1}^{M} \frac{\hbar^{2}}{2 M_{\alpha}} D_{\alpha, k k}, \\
E_{\mathrm{BO}, k}(\boldsymbol{R}) & =E_{\mathrm{e}, k}(\boldsymbol{R})+T_{\mathrm{n}, k}
\end{aligned}
$$

is composed from the electronic energy $E_{\mathrm{e}, k}(\boldsymbol{R})$ which depends on the nuclear coordinates, the kinetic energy of the nuclei $T_{\mathrm{n}, k}$, and two non-adiabatic terms that are represented in terms of the first-order non-adiabatic coupling vector $\boldsymbol{d}_{\alpha, k l}(\boldsymbol{R})=\left\langle\psi_{k}\left|\nabla_{\alpha}\right| \psi_{l}\right\rangle$ and the second-order non-adiabatic coupling coefficient $D_{\alpha, k l}(\boldsymbol{R})=\left\langle\psi_{k}\left|\nabla_{\alpha}^{2}\right| \psi_{l}\right\rangle$. Due to the large difference between electronic and nuclear masses the coupling vectors are of the order of $\left(\frac{m_{\mathrm{e}}}{M_{\alpha}}\right)^{\frac{3}{4}}$ for $\boldsymbol{d}_{k \ell}$ and $\frac{m_{\mathrm{e}}}{M_{\alpha}}$ for $D_{k \ell}$. For example, for a hydrogen atom, i.e. the lightest nuclei, the second-order coupling is around $\frac{1}{1896} \approx 10^{-4}$ [58]. Furthermore, both coupling terms are only have significant contributions to the total energy when two PESs come close together that means at conical intersections. But they arise rarely. Hence, neglecting both terms does not strongly change the value of the total energy and just leads to small errors.

This is the foundation for BOA where the energy $E_{\mathrm{BO}, k}(\boldsymbol{R})$ of the system in the electronic state $k$ is defined by the respective electronic energy and the nuclear kinetic energy as shown in (16). Born and Oppenheimer gave the justification for such a treatment, via expanding energy terms for a molecule in a power series with the fourth root of ratio between electronic and nuclear mass as expanding parameter. This expansion agrees well with energy spacing between electronic, vibrational and rotational motion obtained from spectroscopic experiments [29], [58].

In contrast, the so-called adiabatic approximation leads to the energy $E_{\mathrm{ad}, k}$ as defined by (15) where non-adiabatic coupling terms with $\ell \neq k$ are neglected. Since the electronic wave functions are assumed to be orthonormal, the diagonal elements $\boldsymbol{d}_{\alpha, k k}$ give zeros, so only the diagonal elements of the second-order coupling contribute to $E_{\mathrm{ad}, k}$. Therefore, the adiabatic correction improves the Born-Oppenheimer value of $E_{\mathrm{BO}, k}(\boldsymbol{R})$ by a term of the order of $\leqslant 10^{-4}$. This value tends to decrease as the nuclear masses of the system are increase with respect to the Hydrogen atom.

Both approximations have in common that the motion of the nuclei occurs on a single electronic state PES, which means that there is no transition between electronic states. Most times this single state represents the electronic ground state i.e. the electronic configuration which has the lowest eigenvalue $E_{\mathrm{e}, \ell}(\boldsymbol{R})$. Furthermore, PESs are $3 M$ - 
dimensional functions, so they can be very complex objects when the number of nuclei increases. Therefore, it is very complicated to picture them even for systems where $M$ is a relatively small number. To overcome the complexity in representing PESs one cuts them along 1, 2 or 3 nuclear coordinates which are of interest in the observed process. For example, to look at the adsorption process of $\mathrm{CO}$ at a $\mathrm{Pt}(111)$-surface one cuts the corresponding PES along the center-of-mass (COM)-distance to the surface.

Apart from this, one can choose a different way to represent the wave function: the so-called diabatic representation. Here, the electronic wave function $\psi$ is taken at a fixed set of nuclear positions $\boldsymbol{R}_{0}$ :

$$
\varphi(\boldsymbol{R}, \boldsymbol{r})=\sum_{\ell} \tilde{\chi}_{\ell}(\boldsymbol{R}) \tilde{\psi}_{\ell}\left(\boldsymbol{r} ; \boldsymbol{R}_{0}\right)
$$

where $\tilde{\chi}(\boldsymbol{R})$ is the nuclear and $\varphi(\boldsymbol{r}, \boldsymbol{R})$ is the total wave function. With this ansatz the nuclear and electronic coordinates are really separated, as the electronic wave function $\tilde{\psi}_{\ell}$ does not change with $\boldsymbol{R}$. But $\tilde{\psi}_{\ell}$ is an electronic eigenstate with eigenvalue the $E_{\mathrm{e}, \ell}\left(\boldsymbol{R}_{0}\right)$ of the electronic Hamiltonian in (9) at $\boldsymbol{R}=\boldsymbol{R}_{0}$ :

$$
\widehat{H}_{\mathrm{e}}=\widehat{H}_{\mathrm{e}, \boldsymbol{R}_{0}}+\widehat{V}_{\mathrm{nn}}(\boldsymbol{R})-\widehat{V}_{\mathrm{nn}}\left(\boldsymbol{R}_{0}\right)+\widehat{V}_{\mathrm{ne}}(\boldsymbol{r}, \boldsymbol{R})-\widehat{V}_{\mathrm{ne}}\left(\boldsymbol{r}, \boldsymbol{R}_{0}\right)
$$

Inserting (17) into (8) and multiplying from the left with $\left\langle\tilde{\psi}_{k}\right|$ leads to the following equation:

$$
\begin{aligned}
\left\langle\tilde{\psi}_{k}|\widehat{H}| \varphi\right\rangle & =\widehat{T}_{\mathrm{n}} \tilde{\chi}_{k}(\boldsymbol{R})+\sum_{\ell}\left\langle\tilde{\psi}_{k}\left|\widehat{H}_{\mathrm{e}}\right| \tilde{\psi}_{\ell}\right\rangle \tilde{\chi}_{\ell}(\boldsymbol{R}) \\
& =\widehat{T}_{\mathrm{n}} \tilde{\chi}_{k}(\boldsymbol{R})+\sum_{\ell} V_{k \ell}\left(\boldsymbol{R}, \boldsymbol{R}_{0}\right) \tilde{\chi}_{\ell}(\boldsymbol{R}),
\end{aligned}
$$

where $T_{\mathrm{n}}$ is just applied to nuclear wave function of state $k$ and the relation (18) is used. The expectation value of $\widehat{H}_{\mathrm{e}}$ leads to the diabatic matrix element $V_{k \ell}\left(\boldsymbol{R}, \boldsymbol{R}_{0}\right)$ which depend on both sets of nuclear coordinates $\boldsymbol{R}_{0}$ and $\boldsymbol{R}$. So all information regarding nonadiabaticity is contained in the matrix element $V_{k l}(\boldsymbol{R})$, because it describes coupling to all other electronic states.

Adiabatic and diabatic representation can be seen as limit cases for considering the electronic motion. In the adiabatic representation the electrons are so fast that they immediately adjust their positions that the energy $E_{\ell}(\boldsymbol{R})$ of the electronic state $\psi_{\ell}(\boldsymbol{r} ; \boldsymbol{R})$ is minimized when the nuclei reach their positions $\boldsymbol{R}$. So from a nuclear point of view the electrons are always in the ground state.

Whereas in the diabatic representation the electrons move so slowly that they do not have enough time to adjust their positions, so the electrons are always in same con- 
figuration as described $\tilde{\psi}_{\ell}\left(\boldsymbol{r} ; \boldsymbol{R}_{0}\right)$ which corresponds to the set of nuclear coordinates $\boldsymbol{R}_{0}$. Thus changes in the in electronic energy due to the actual nuclear configuration $\boldsymbol{R}$ are introduced by the diabatic matrix element. It is possible to transform between these representations by transforming either the electronic or the nuclear wave function via the so called adiabatic to diabatic transformation [53]. Since the picture of the electronic and nuclear motion, on which the adiabatic representation is based, applies to most situations in chemistry, this representation is more often used than the diabatic one.

Further the concept of a single PES is often useful when calculating energies and derived properties of molecules like spectra and optimized structure but also to identify reaction characteristics such as transition states and subsequently reaction rates. But there are also examples where interactions between molecules or atoms cannot be explained within this picture that nuclei moving on a single PES provided by a fixed electron configuration. This is where the BOA breaks down and both or one of the non-adiabatic coupling terms deliver a substantial contribution to the total energy. The first-order nonadiabatic coupling vector is large when the electronic environment changes drastically with a small change in the nuclei coordinate. This is the case $e$.g. in a photo-reaction, where a molecule is electronically excited by radiation with an appropriate energy. The second non-adiabatic coupling coefficient arises when one electronic state is not enough to describe the wave function of the system. This happens for example at the dissociation of halides in gas phase, e.g. lithium fluoride (LiF). Since the PES describing the ionic state leads to a more stable configuration when the diatomic has its equilibrium distance, but when the molecule is dissociated then the neutral atoms are more stable than the ionic species, that the neutral PES leads to a lower energy. Hence two states are neccessary to describe the dissociation process. When both PESs come close to each other adiabatic approximation fails and the non-adiabatic coupling increases. This is in the region of the avoided crossing and the conical intersection [53, 59].

\subsection{Density functional theory}

In the previous section we have seen that the nuclear motion can be described on a single electronic PES $E_{\mathrm{e}, 0}(\boldsymbol{R})$, which is the energy of the electronic ground state. This section describes how we can obtain it. One approach was already mentioned before, namely solving Eq. (10) with an appropriate ansatz for the wave function. This approach is mostly used in theoretical chemistry for small molecules. Another approach, which has its roots in solid state physics, is DFT. This theory has the advantage that it can handle systems with large numbers of electrons and nuclei like metal surfaces or large 
biomolecules with 100s or 1000s of atoms. This is not possible with wave function based methods because a wave function depends on $3 N$ coordinates. DFT avoids using such a complex function, and instead uses the electronic density $\rho(\boldsymbol{r})$ to determine the energy of the considered system. Since the density depends on just three spatial coordinates it is less complex than a wave function. In practice, it is easier to determine the electron density at certain points in space than to look for the positions of all electrons in the system, especially when the number of electrons in the system is very large. Furthermore, the density is an observable which can be measured with X-ray techniques and expressed as square of the absolute value of the wave function [55]. In this theory the energy is a functional of the electron density. A functional maps a function onto a number, whereas a function maps a number onto another number. An example for the former is the definite integral of a function. So the electron density $\rho$ is a function of the spatial coordinates. But to obtain the electronic energy we need to integrate the density over the whole space. Thus it is a functional of $\rho$. I will use the common notation $F[f(x)]$ for a functional, where $f$ is the function depending on the variable $x$, and $F$ is the corresponding functional. But why can we connect energy and electron density in this way?

Both in classical and quantum mechanics, the Hamiltonian determines the system. The Hamiltonian depends on the positions and charges of all electrons and nuclei. An intuitive proof how the electron density delivers us all the necessary information was made by E. Bright Wilson jr. who said that the integral of the density is equal to the number of electrons, the density's cusps give the nuclear positions, and the amplitudes of these cusps deliver the charges of the nuclei $[54,60]$. Hence, the electronic density completely defines the system's Hamiltonian and so its energy. However, as we have to minimize the energy with respect to the density we have to ensure that the minimum energy is described by a unique density.

A proof that the ground state electronic density $\rho_{0}$ uniquely describes the ground state energy $E_{\mathrm{e}, 0}$ was first given by Hohenberg and Kohn [61]. They assumed that two different electron densities give the same ground state energy and showed via variation principle that this is wrong. This is a proof by reductio ad absurdum.

The energy functional of the electron density, in equivalence to the electronic Hamiltonian in (9), can be written as

$$
\begin{aligned}
E[\rho(\boldsymbol{r})] & =T[\rho(\boldsymbol{r})]+V_{\mathrm{ne}}[\rho(\boldsymbol{r})]+V_{\mathrm{ee}}[\rho(\boldsymbol{r})] \\
& =T[\rho(\boldsymbol{r})]+V_{\mathrm{ne}}[\rho(\boldsymbol{r})]+J[\rho(\boldsymbol{r})]+V_{\mathrm{X}}[\rho(\boldsymbol{r})],
\end{aligned}
$$


where $T[\rho(\boldsymbol{r})]$ is the kinetic energy functional, $V_{\text {ne }}[\rho(\boldsymbol{r})]$ is the functional for the nuclear-electron attraction and $V_{\mathrm{ee}}[\rho(\boldsymbol{r})]$ its counterpart for electron-electron repulsion. The last term can be divided in a Coulomb part $J[\rho(\boldsymbol{r})]$, which describes the classical electron-electron repulsion, and an exchange part $V_{\mathrm{X}}[\rho(\boldsymbol{r})]$. The term $V_{\text {ne }}[\rho(\boldsymbol{r})]$ can be described by the classical Coulomb expression where the nuclear attraction to the electron density $\rho(\boldsymbol{r})$ is integrated over the whole space. As we now have established a relation between the electron ground state density and the corresponding energy, we need to know the dependence of each contribution of Eq. (20) on the density. In, the case of the uniform electron gas (ueg) model it is possible to derive expressions for most of the terms in (20) by using statistical methods as described by Fermi and Dirac [62, 63]. They considered the probability distributions of particle positions and velocities, under the prerequisite that the particles follow the Pauli principle [64], to find the minimum energy of the particles as functional of their density.

In this model electrons form a gas which contains an infinite number of interacting particles in an infinite volume, so that the density as well as the energy per particle is finite. To make the overall system electronically neutral the electrons are surrounded by the same number of positive charges. This is important to suppress the long-ranged Coulomb interaction which leads to a divergence of the energy per particle [65]. This electronically neutral ueg is also called jellium. Further, two electrons with different spins can occupy a cell, like an orbital, with a volume of $h^{3}$ within the phase space, i.e. momentum and spatial space. When the electrons come to their potential energy minimum they occupy a spherical volume in momentum space. But as the electron description is based on statistics, their individual behavior is not captured [66]. Therefore, correlation - how the motion and the position of one individual particle influences the motion and position of a second one - is neglected.

The first attempt for a description of the energy functional in terms of the electron density was given by Thomas and Fermi [67, 68], who used the ueg model as described above. Later on the model was expanded to an exchange part by Bloch and a few years later by Dirac too, where both using two plane waves to describe the interaction between two electrons [69, 70]. The so obtained energy functional for the mean energy of an uniform electron gas can be written as

$$
\begin{aligned}
E_{\mathrm{TFD}}[\rho(\boldsymbol{r})] & =T_{\mathrm{TF}}[\rho(\boldsymbol{r})]+V_{\mathrm{X}, \mathrm{D}}[\rho(\boldsymbol{r})]+V_{\mathrm{ne}}[\rho(\boldsymbol{r})] \\
& =-\frac{3 h^{2}}{40 m_{\mathrm{e}}}\left(\frac{3 \rho(\boldsymbol{r})}{\pi}\right)^{\frac{2}{3}} \rho(\boldsymbol{r})-e^{2} \frac{3}{4}\left(\frac{3 \rho(\boldsymbol{r})}{\pi}\right)^{\frac{1}{3}} \rho(\boldsymbol{r})+V_{\mathrm{ne}}[\rho(\boldsymbol{r})],
\end{aligned}
$$


where $T_{\mathrm{TF}}[\rho(\boldsymbol{r})]$ is the kinetic energy functional found by Thomas and Fermi $[67,68]$ and $V_{\mathrm{X}, \mathrm{D}}[\rho(\boldsymbol{r})]$ is the exchange part derived by Dirac [70].

Despite the fact that DFT is in principle a wave function free theory it has been shown that the introduction of orbitals increases the description of $T[\rho(\boldsymbol{r})]$ and clearly provides better results for molecular systems. The idea of using orbitals in DFT was brought up by Kohn and Sham [71]. Within this framework, which is similar to the HartreeFock method, atoms can form stable molecules in DFT. Both, the orbital-free and the Kohn-Sham DFT have in common that they formulate the expression for the exact energy functional $E[\rho(\boldsymbol{r})]$ as the sum of energies of non-interacting electrons and their differences to the exact functional, or to be more precise terms which are exactly known and parts which are not known. The latter are summed up in the exchange-correlation energy functional $E_{\mathrm{XC}}[\rho(\boldsymbol{r})]$. So one can write the total energy functional $E[\rho(\boldsymbol{r})]$ as

$$
E[\rho(\boldsymbol{r})]=T_{\mathrm{S}}[\rho(\boldsymbol{r})]+V_{\mathrm{ne}}[\rho(\boldsymbol{r})]+J[\rho(\boldsymbol{r})]+E_{\mathrm{XC}}[\rho(\boldsymbol{r})]
$$

Here, $T_{\mathrm{S}}$ stands for the kinetic energy functional as calculated with non-interacting Kohn-Sham orbitals or the corresponding functional by Thomas and Fermi as given in (21). The last term

$$
E_{\mathrm{XC}}[\rho(\boldsymbol{r})]=T[\rho(\boldsymbol{r})]-T_{\mathrm{S}}[\rho(\boldsymbol{r})]+V_{\mathrm{ee}}[\rho(\boldsymbol{r})]-J[\rho(\boldsymbol{r})],
$$

contains both the kinetic correction of $T_{\mathrm{S}}$ to the real kinetic energy and the reduction of the Coulomb repulsion by the quantum mechanical effect of exchange and the electronelectron correlation, which is the real electron-electron interaction $V_{\mathrm{ee}}[\rho(\boldsymbol{r})]$ of the system. While the principle definition of $E_{\mathrm{XC}}[\rho(\boldsymbol{r})]$ is clear, its actual form has been under investigation over the last decades. The next section describes some attempts to approximate the effects of exchange and correlation within DFT. But before this I describe these quantum mechanical effects in more detail.

\subsubsection{Exchange and Correlation}

In this section I describe the origin of the quantum mechanical effects of exchange and correlation, as these effects give insights as to how we can define approximations in order to get to the right $E_{\mathrm{XC}}[\rho(\boldsymbol{r})]$. The exchange effect arises from the Pauli principle [64]. It is one of the pillars of quantum mechanics and states that the total wave function of fermions must be anti-symmetric, i.e. electrons with the same spin cannot occupy the same cell in phase space. So exchange of two electrons between different cells in phase space (orbitals) leads to a different sign in the total wave function, and, moreover, 
only electrons with the same spin contribute to exchange energy. This contribution is negative and can be seen as a classical correction to the Coulomb repulsion because electrons with the same spin try to avoid each other due to the Pauli principle. The electron-electron correlation is the direct interaction of two particles at positions $\boldsymbol{r}_{1}$ and $\boldsymbol{r}_{2}$. Due to the same electronic charge electrons want to avoid each other, and so the probability of finding an electron close to another one must decrease. This effect is known as Coulomb correlation. In contrast to the exchange correlation it is completely independent of the spin state of electrons. Since the electron density gives an answer to the question of how likely it is to find an electron in a particular volume element, one can use it to extend this concept and ask what is the probability of finding an electron pair in the two volume elements $d^{3} \boldsymbol{r}_{1}$ and $d^{3} \boldsymbol{r}_{2}$. This is expressed by the pair density of two particular electrons $\rho_{2}\left(\boldsymbol{r}_{1}, \boldsymbol{r}_{2}\right)$ which depends on the density in both coordinates, in the spins and contains all information about electron correlation, and hence it gives in principle all information necessary to find the exchange-correlation functional.

Similar to the procedure of separating the energy functional in a part which can be calculated exactly and a part which is unknown we can divide the probability of finding a second electron at a certain position, when we know the position of a first electron, in two parts. One part is the completely uncorrelated or independent probability which is the product of the probability of finding an electron at two different positions $\rho\left(\boldsymbol{r}_{1}\right) \rho\left(\boldsymbol{r}_{2}\right)$, and the other part is the conditional probability. So the pair density can be expressed as

$$
\rho_{2}\left(\boldsymbol{r}_{1}, \boldsymbol{r}_{2}\right)=\rho\left(\boldsymbol{r}_{1}\right) \rho\left(\boldsymbol{r}_{2}\right)+\rho\left(\boldsymbol{r}_{1}\right) h_{\mathrm{XC}}\left(\boldsymbol{r}_{1}, \boldsymbol{r}_{2}\right),
$$

where $h_{\mathrm{XC}}\left(\boldsymbol{r}_{1}, \boldsymbol{r}_{2}\right)$ is known as the exchange-correlation hole, and it gives the reduced probability of finding an electron at $\boldsymbol{r}_{2}$ when one electron is at $\boldsymbol{r}_{1} . h_{\mathrm{XC}}\left(\boldsymbol{r}_{1}, \boldsymbol{r}_{2}\right)$ is the difference between the pair density $\rho_{2}\left(\boldsymbol{r}_{1}, \boldsymbol{r}_{2}\right)$ dived one particle density $\rho\left(\boldsymbol{r}_{1}\right)$ and subtracted from one particle density $\rho\left(\boldsymbol{r}_{2}\right)$. The hole describes the position which cannot occupied by the second electron, because this the position is already occupied by the first electron. The hole term can be separated into an exchange part, the Fermi hole and into a correlation part, the Coulomb hole. The separation is justified because of the different origin of exchange and correlation. For example the exchange is between electrons with the same spin whereas correlation occurs between electrons with the same and opposite spin. But only the total hole has a physical meaning and it gives -1 when integrated over $\boldsymbol{r}_{2}$.

In the next subsection, I describe some approximations for the exchange-correlation functional. 


\subsubsection{Approaches to the exchange-correlation functional}

The first approximation to describe exchange-correlation energy is the local-density approximation (LDA). A central role in this approximation involves the model of the ueg, which was described previously. Real systems which come close to this model system are metals and insulators like sodium chloride in which at least the valence electrons are distributed over all atoms. For this model system it is possible to calculate the contribution of the exchange $V_{\mathrm{X}}[\rho(\boldsymbol{r})]$ to energy functional, as written in Eq. (21). Although this was derived by Bloch and Dirac in the late 1920s already [69, 70], it is commonly associated with Slater [72]. Even within the model of the ueg the correlation energy $E_{\mathrm{C}}[\rho(\boldsymbol{r})]$ could not be obtained analytically. It can however be calculated numerically, as was done by Wigner in the mid-1930s using the second-order perturbation theory with an accuracy of $20 \%$ [73]. Calculations to a very accurate level were done by Ceperley and Alder using quantum Monte-Carlo calculations [74]. But also other methods, as the random-phase approximation etc., were considered to calculate $E_{\mathrm{C}}[\rho(\boldsymbol{r})]$ for different densities. The calculated values of the correlation energy are fitted to different functions producing a lot of different XC-functionals [75, 76].

The consideration of the uniform electron gas can be easily extended to a gas which is built up by two spin densities $\rho_{\alpha}$ and $\rho_{\beta}$, which sum up to the total density $\rho$. This spin-polarized gas is characterized by the parameter $\zeta=\frac{\rho_{\alpha}-\rho_{\beta}}{\rho}$. The relative spinpolarization. $\zeta$ and the Wigner-Seitz radius $r_{s}=\left(\frac{3 \rho}{4 \pi}\right)^{\frac{1}{3}}$, i.e. the effective radius of an electron, are often used to describe the exchange-correlation energy of the particle. The exchange-correlation functional within the so-called local-spin-density approximation (LSD) is given by

$$
E_{\mathrm{XC}}^{\mathrm{LSD}}\left[\rho_{\alpha}(\boldsymbol{r}), \rho_{\beta}(\boldsymbol{r})\right]=\int \rho(\boldsymbol{r}) \epsilon_{\mathrm{XC}}^{\mathrm{ueg}}\left[\rho_{\alpha}(\boldsymbol{r}), \rho_{\beta}(\boldsymbol{r})\right] d^{3} \boldsymbol{r},
$$

where $\epsilon_{\mathrm{XC}}^{\mathrm{ueg}}\left[\rho_{\alpha}(\boldsymbol{r}), \rho_{\beta}(\boldsymbol{r})\right]$ is the exchange-correlation energy per particle of the ueg. Eq. (25) can be summarized in the following statement: Within the LDA or LSD one approximates firstly that the exchange-correlation energy at a certain point of the system just depends on the electron density at this point $\epsilon_{\mathrm{XC}}\left[\rho_{\alpha}(\boldsymbol{r})\right]$ and secondly that its value at position $\boldsymbol{r}$ is equal to the energy of the ueg with the same density. How good the approximations are depends on how close a real system comes to the model of the ueg. Since metals and insulator are well described by the ueg the LDA gives good results; sometimes even better than the wave function methods for those systems, which makes it very popular in solid-state physics. 
However, because atoms and molecules are not well described by the ueg model, the LDA gives very bad results for atomic energy or binding energies. A clear difference between an atom and the ueg is that the density in an atom changes over space, so the density decreases far away from the nucleus and increases close to it.

Therefore the next approximation (or step on Jacob's ladder as said by Perdew) to come closer to the real XC functional is the generalized-gradient approximation (GGA). In this framework one assumes that $\epsilon_{\mathrm{XC}}$ depends locally not only on the electron density at $\boldsymbol{r}$ but in addition on its gradient $\nabla \rho(\boldsymbol{r})$. So the GGA-XC functional for a spin-polarized system within this approach is

$$
E_{\mathrm{XC}}^{G G A}\left[\rho_{\alpha}(\boldsymbol{r}), \rho_{\beta}(\boldsymbol{r})\right]=\int \epsilon_{\mathrm{XC}}^{\mathrm{ueg}} F\left(\rho_{\alpha}(\boldsymbol{r}), \rho_{\beta}(\boldsymbol{r}), \nabla \rho_{\alpha}(\boldsymbol{r}), \nabla \rho_{\beta}(\boldsymbol{r})\right) d^{3} \boldsymbol{r},
$$

where $F$ is a function of $\alpha$ - and $\beta$-spin densities and their gradients. The gradient approach was used by Weizsäcker [77] for the kinetic energy functional to successfully describe processes in atomic nuclei. But that did not improve the description of molecules. In the same way as for the LDA-XC, the function $F$ has to be fitted to facilitate practical calculations. The choice of the fitting function is central in generating a XC-functional and therefore the quest of the Holy Grail within DFT.

In the last decades much effort was spent on finding a good parameterization for $F$ which led to a variety of different expressions and therefore to many functionals [78-82]. In principle there are two philosophies on the construction of these functionals. One is based on the foundation that the constructed functional as well as the parameterized function fulfill the conditions derived from physical properties for the XC-hole. For example, the total hole $h_{\mathrm{XC}}$ must yield -1 , thus the correlation hole vanishes when integrated over the whole space, as stated in the previous section (2.2.1); or when the density is varying slowly, $F$ should approach 1 to obtain the ueg result. Further conditions can be found in [83], though no developed functional fulfills them all. This approach can be seen as ab initio-like due to its physical foundation. The Perdew and Wang of 1991, a GGA functional (PW91) [76] and the Perdew Burke Ernzerhofer, a GGA functional (PBE)-functional [83] developed in the early 1990s follow this route. Also, the revised Perdew Burke Ernzerhofer, a GGA functional (RPBE)-functional [84] on the GGA-level relies on the first philosophy, but here the exchange part does not fulfill the sum rule for the total hole [54].

The second philosophy is a semi-empirical approach. Here one tries to parameterize the functions $F$ so that the obtained results match experimental data for a variety of properties e.g. lattice constants or molecular binding energies. 
In GGA the exchange and correlation energy contributions are often treated independently when constructing functionals. Therefore functionals are generated which contain the exchange part from an earlier developed functional and the correlation from another one and vice versa. This is true for one of the most popular GGA-functionals, the BLYP, which contains the exchange of Becke's functional from 1988 and the correlation of the functional by Lee, Yang and Parr [78, 85].

Since the GGA-functionals PW91 and RPBE are mostly used for calculations carried out in this thesis, they are described in qualitative way. PW91 has a relative complicated form and depends on many parameters compared to RPBE. In both cases exchange and correlation energy are treated separately. The construction of RPBE was motivated by the fact that PW91 and PBE give similar results for energies of atoms and molecules but deliver more deviating results for adsorption energies, so RPBE focus on the latter [84]. Therefore RPBE delivers adsorption energies of atoms and molecules on surfaces which are closer to experimental values. Whereas PW91 gives better results for other properties e.g. the obtained lattice constants are closer to the experimental values than those calculated with RPBE. The reason that PW91 and PBE give similar results for a lot of properties is that PBE is based on PW91 but has a simpler functional form i.e. PBE has less parameters than PW91. Hence, we can say that the PBE functional is more general than PW91.

Finally, there are higher steps on Jacob's ladder to the true XC-functional than GGA: as meta-GGA — like B95 [86] or M06-L [87] — or hybrid-functionals like the very popular B3LYP $[88,89]$. However, these functionals are computationally more demanding than GGA and for this reason they are not used in this thesis.

\subsection{Electrons in periodic systems and treatment of electronic occupation of metallic bands}

In case of periodic systems like solids and surfaces the potential energy is periodic and thus the Hamiltonian is too. So, to solve the electronic Schrödinger equation (10) appropriate eigenfunctions are needed which exhibit periodicity. The most common ansatz for these functions is associated with Bloch [90], who stated that the electronic wave function is given by

$$
\psi_{\boldsymbol{k}}(\boldsymbol{r})=u_{\boldsymbol{k}}(\boldsymbol{r}) \exp i \boldsymbol{k} \boldsymbol{r}
$$

where $u_{\boldsymbol{k}}(\boldsymbol{r})$ is a periodic function with the same periodic boundaries as the considered system and $\boldsymbol{k}$ is the momentum vector of the wave. As the eigenfunctions are periodic, this property also applies to their eigenvalues and thus the corresponding energy. As 
the wave functions and the energy are repeated periodically over the whole structure a picture of electronic bands is more appropriate than a picture of orbitals which describes electrons in an atom or a molecule. Therefore it is enough to consider the smallest building block, referred to as unit cell, which builds up the whole periodic system to solve the electronic structure of the total system. Furthermore, instead of describing the electronic structure in real space it is better to solve the Schrödinger equation in reciprocal space $\boldsymbol{k}$, where the unit cell is called inner Brillouin zone (IBZ). The change from one space to the other is done by (Fast-)Fourier-Transformation. So, to obtain the electronic ground state energy $E_{\mathrm{e}, 0}(\boldsymbol{k})$ one has to integrate one-electron and manybody terms over all electronic bands within the IBZ. For insulators and semi-conductors integration can be carried easily, because the function in these cases is many times differentiable as the occupied and unoccupied bands are separated by a large energy gap. But for metals this is not the case, and the function describing the electronic occupation is a step function, which plummets from 1 to 0 at the Fermi energy and is not easy to integrate. Moreover, the bands are just partially filled with electrons, and so the function is discontinuous. This leads to a very slow convergence when taking the integrals for metallic systems.

To obtain better convergence and faster integration one can replace the step function by a function which describes the electronic occupation not at $0 \mathrm{~K}$ but at a finite temperature $e$.g. the Fermi distribution which is characterized by a width $\sigma=k_{B} T$ [91]. A more sophisticated approach for the step function is the expansion of the Gaussian function, which was before used [92], in terms of Hermite-polynomials as proposed by Methfessel and Paxton [93]. For this approach the choice of the width $\sigma$ does not influence the resulting true energy value $E(\sigma \rightarrow 0)$ as in the case of a Fermi function. The choice of the smearing function describes the electronic occupation and so gives the expression for the electronic entropy $S$. With the introduction of temperature one has to minimize the free energy $F$ instead of the energy $E$ :

$$
E=F+T S=F+\sum_{\boldsymbol{k}, n} \sigma f(n, \epsilon(\boldsymbol{k}))
$$

where $S$ can be obtained from the electronic occupation $f(n, \epsilon(\boldsymbol{k}))$ by summing over all $\boldsymbol{k}$-points and bands $n$. Here $\epsilon(\boldsymbol{k})$ denotes the energy of the band. The following considerations are based on [94]. With $F$ and $E$ one can obtain an expression for the energy at $0 \mathrm{~K} E(\sigma \rightarrow 0)=E_{0}$. Using $S=-\frac{d F}{d \sigma}$ and that the deviation of $F$ from $E_{0}$ is of $N+2$ order in $\sigma$ for a Methfessel-Paxton (MP) smearing function of $N^{t h}$-order, one gets:

$$
F=E_{0}+\gamma \sigma^{2+N}
$$


Inserting (29) in (28) one obtains

$$
E_{0}=\frac{(N+1) F+E}{N+2},
$$

where $N$ is 0 in the case of the Gaussian smearing [92], when free energy $F$ is a quadratic function of $\sigma$.

\subsection{Ab initio molecular dynamics}

MD is a method to simulate the motion of molecules, which consists of two building blocks: (i) the calculation of energies and forces and (ii) equations governing the motion (a propagation scheme). For (i) a PES calculated beforehand is often used. Unfortunately, there is no PES - or only an incorrect one - available for the systems investigated in this thesis and the energy has to be determined. In the framework of AIMD the energies and forces are calculated by solving the electronic stationary Schrödinger equation at each time step during the propagation ("on the fly") by either DFT or wave-function methods. To tackle the building block (ii), the atoms are considered as point-shaped classical particles, and the laws of classical mechanics are applied. In most cases Newton's second law is used to describe the motion of the particles. But also other formulations of classical mechanics like that of Lagrange or Hamilton are applicable, depending on the coordinate system or other considerations. Hamilton mechanics, for example, has the advantage that the total energy $H$ is the central quantity whereas in the Lagrangian one it is the difference between kinetic and potential energy $L=T-V$ [95]. As Newton's second law states, the motion of a particle $\alpha$ is governed by the force acting on this particle

$$
\boldsymbol{F}_{\alpha}=M_{\alpha} \boldsymbol{a}_{\alpha}=M_{\alpha} \frac{\mathrm{d}^{2} \boldsymbol{R}_{\alpha}}{\mathrm{d} t^{2}}=-\frac{\partial V(\boldsymbol{R})}{\partial \boldsymbol{R}_{\alpha}},
$$

where $M_{\alpha}$ is the mass of the particle and $\boldsymbol{a}_{\alpha}$ is the acceleration of the particle. The last term of (31) defines how $\boldsymbol{F}_{\alpha}$ can be obtained from the potential energy of the system with respect to the position of the particle $\boldsymbol{R}_{\alpha}$. Eq. (31) is also obtained from the canonical Hamilton equations for general coordinates, whereas Newton's formalism remains in Cartesian coordinates. Here, the system is conservative meaning that the total energy of the system is conserved and $V(\boldsymbol{R})$ depends only on nuclei positions and not on their derivatives in time. How to get force is of most importance for MD and will be described below.

Eq. (31) infers that we must integrate the acceleration over time to get the first derivative of position with respect to time, i.e. the velocity $\boldsymbol{v}_{\alpha}$. A further integration in time leads 
to the position of the particle $\boldsymbol{R}_{\alpha}$ as a function of time. In the case of the system with many degrees of freedom, the analytical integration of Eq. (31) is not possible, so one has to choose a finite time step to do the numerical integration. For this purpose, a large number of algorithms is available.

The algorithm of choice should satisfy certain requirements which affect its stability and performance. The algorithm has to be [95]:

1. time-reversible, meaning that integration over a negative time step leads to the positions of the previous time step;

2. simplectic, meaning that the phase space must be constant;

3. conservative, meaning that the total energy of the system does not change with time $\left(\frac{d H}{d t}=0\right)$

The simplest approach to approximate the time-dependence of the position of a particle $\boldsymbol{R}_{\alpha}$ is to use a Taylor series. But this so called Euler-ansatz is not time-reversible and also performs badly on the energy conservation. An algorithm that has a good energy conservation and is time-reversible is the Verlet-algorithm [96]. A problem with the Verlet-algorithm is that it does not contain the velocity which is necessary to determine the kinetic energy. Furthermore, at the initial time step approximations are needed to estimate the previous positions. To overcome this issue it is possible to formulate the Verlet-scheme in a way that gives positions and velocities at the same time. This formulation looks similar to a Taylor-series expansion in the coordinates, however the velocities of the following time step are obtained differently to those in the Verletscheme. The velocity-Verlet algorithm updates positions and velocities as follows [56, 97]:

$$
\begin{gathered}
\boldsymbol{R}_{\alpha}(t+\Delta t)=\boldsymbol{R}_{\alpha}(t)+\boldsymbol{v}_{\alpha}(t) \Delta t+\frac{1}{2} \frac{\boldsymbol{F}_{\alpha}(t)}{m_{\alpha}} \Delta t^{2} \\
\boldsymbol{v}_{\alpha}(t+\Delta t)=\boldsymbol{v}_{\alpha}(t)+\frac{\boldsymbol{F}_{\alpha}(t)+\boldsymbol{F}_{\alpha}(t+\Delta t)}{2 m_{\alpha}} \Delta t
\end{gathered}
$$

where $\boldsymbol{R}_{\alpha}(t), \boldsymbol{v}_{\alpha}(t), \boldsymbol{F}_{\alpha}(t)$, and $\boldsymbol{R}_{\alpha}(t+\Delta t), \boldsymbol{v}_{\alpha}(t+\Delta t), \boldsymbol{F}_{\alpha}(t+\Delta t)$, are the position, velocity and force of nucleus $\alpha$ at the current $t$ and the next $t+\Delta t$ time step, respectively. This algorithm, which is used in Fritz-Haber-Institute ab initio molecular simulations (FHI-aims), has a very good stability with respect to the total energy, i.e. produces rather small drifts with moderately small time steps a over long simulation time. It can be shown that both velocity- and (position-)Verlet-algorithm are equivalent with respect to positions, so they lead to at least very similar trajectories but to different total energies, due to different velocities and therefore kinetic energies. Other algorithms 
which are based on higher orders of the derivatives of the particle coordinates, so called predictor-corrector methods like the Gear-algorithm $[98,99]$ used in Vienna Ab initio Simulation Package (VASP) [100], need more computational memory and have a better total energy stability within short times. But these algorithms perform worse than Verletlike algorithms on long-term energy stability and also on the two other requirements mentioned above [56, 101].

Both Verlet-like and predictor-corrector algorithms need the force acting on every particle, so the gradient of the interaction energy $V(\boldsymbol{R})$ for all particles is needed, to compute the positions and velocities of the next time step.

To obtain these quantities one has to find the eigenstates of the electronic Hamiltonian in (9). Therefore, in AIMD the electronic structure problem for the given nuclear configuration is solved within the BOA. So, the minimum energy with respect to the wave function is determined to find the ground state energy. From this the force acting on each nucleus is calculated by the numerical derivative of the energy with respect to the nuclear positions. The Hellman-Feynman theorem [102, 103] states

$$
\frac{\partial E_{\mathrm{e}}}{\partial \boldsymbol{R}}=\frac{\partial}{\partial \boldsymbol{R}}\left\langle\psi_{k}\left|\widehat{H}_{\mathrm{e}}\right| \psi_{k}\right\rangle=\left\langle\psi_{k}\left|\frac{\partial \widehat{H}_{\mathrm{e}}}{\partial \boldsymbol{R}}\right| \psi_{k}\right\rangle .
$$

But the theorem is true only in case of a complete (infinite) basis set for the wave function and a self-consistent Hamiltonian. Both conditions are never fulfilled in practice because on one hand the minimization of energy with respect to the wave function is aborted when a certain criterion is reached, and on the other hand the number of basis functions is finite. Still, using Eq. (34) the forces are approximately calculated to determine the positions and velocities for the next time step.

The determination of the electronic ground state energy can be done - depending on the system size - either with wave function methods or for larger systems ( $>100$ atoms) with DFT which basics are described in 2.2. This reveals also the biggest disadvantage of AIMD. Since at every time step the electronic energy has to be determined selfconsistently it is computationally very demanding. 


\section{Methodology}

In this Chapter I will introduce the methods I used within this thesis. First, the software packages VASP [104] and FHI-aims [105] used to perform DFT calculations and AIMD simulations will be described. In particular, I will focus on the difference between them so that the choice of the appropriate code in a certain situation later on can be better understood. After discussing these programs and some of their features which were the main working horses I will introduce two other programs which I used to a smaller extent. The first one is Phonopy $[106,107]$ which was used to calculate phonon-spectra of different metal crystals and surfaces. The second one is the Bader charge analysis by Henkelman [108], based on Bader's Quantum Theory of Atoms in Molecules [109], which I used for analyzing charges on atoms. Then in the last section I will describe the procedure to find a MEP.

\subsection{Vienna ab initio simulation package}

VASP is a computer program which performs electronic structure calculations and AIMD and was developed at the University of Vienna. To start a calculation in VASP one needs four input files. I will describe their structure and purpose in the following subsections.

\subsubsection{INCAR-file}

The first file is named INCAR, for which an example is shown in Fig 1, it contains all parameters which control the calculation in general, e.g. which kind of calculation should be done: AIMD simulation, structure optimization/relaxation or calculation of the energy for a fixed geometry. This is defined by a "tag" which has a certain value. For example is an AIMD simulation requested by writing IBRION=0. For the majority of these tags default values exist so that no adjustment by the user is required. An example for an INCAR file is given in Tab. With IBRION the nuclear motion is controlled, and a value of -1 keeps the structure fixed, whereas a value of 1 of 2 is set for a structure relaxation. Other tags define, for example, which XC-functional should be used, how the spin of the 
system is treated, the details concerning the optimization/relaxation of the electronic structure etc. Of particular importance are the tags influencing the minimization procedure for the electronic energy $E_{\mathrm{el}}$ and, therefore, extensive convergence tests have to be performed. So, one should always test the tags ISMEAR and SIGMA which control the treatment of the electronic occupation of orbitals or bands (see section 2.3) as well as ENCUT which gives the cut-off energy $E_{\text {cut }}$ of plane waves included in the basis set wave function, i.e. $E_{\text {cut }}$ defines the size of the basis set. This is the most parameter when doing calculations and it has to be checked very carefully. For the optimization of the electronic wave functions, there are the block-Davidson (DAV) $[110,111]$ and the RMM-DIIS-scheme [112] available. The former is more robust and is the default value for the ALGO-tag. In this thesis mostly a mix of the two schemes is used (ALGO=F), which is fairly robust choice. For systems where convergence of the electronic optimization is difficult to reach, tags to control initial (MAGMOM) and total (NUPDOWN) magnetic moments of the individual species and/or the total system may be varied as well as the tags to control the mixing of the old and the new electron density (AMIX) and the new and old spin density (AMIX_MAG) within the self-consistent-field cycle. Furthermore, in the INCAR the output of the program is defined, like the CHARGCAR and WAVECAR-files, containing the self-consistent valence electron density and wave function, respectively.

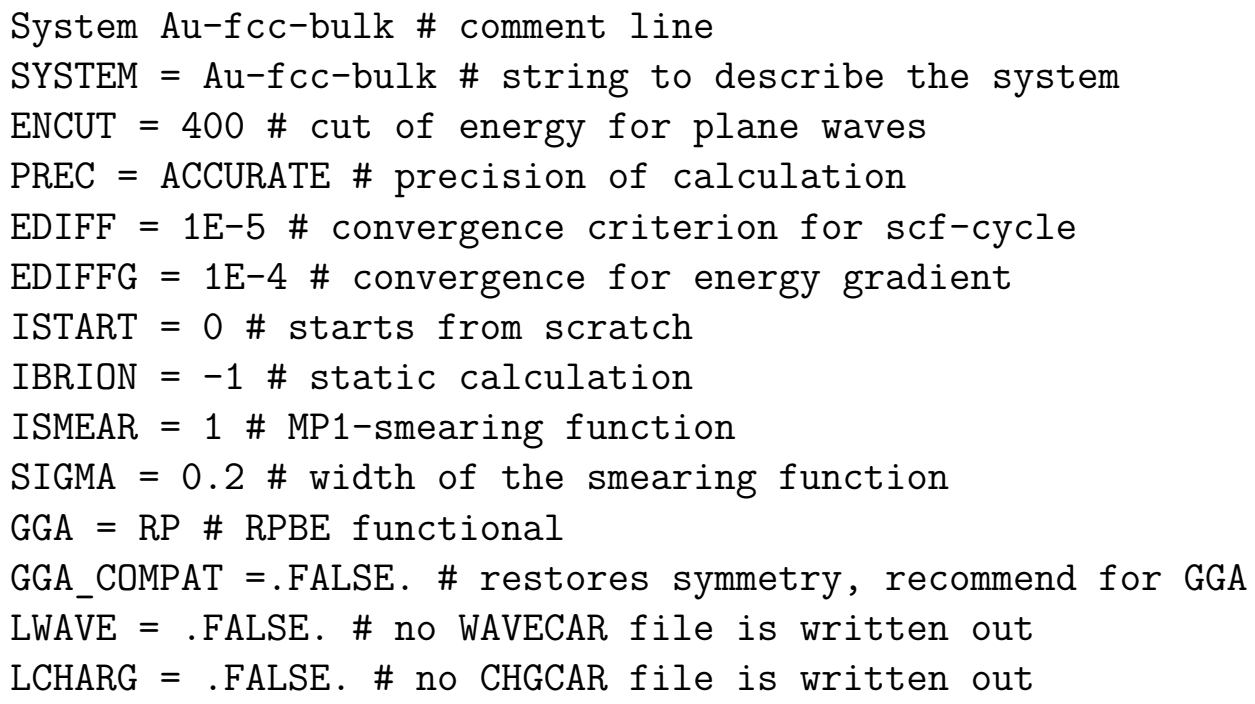

Fig. 1: Exemplary INCAR-file of an Au bulk calculation.

\subsubsection{KPOINTS-file}

The second file is the KPOINTS, for which an example is shown in Fig. 2. As the name implies it contains all information about the $\boldsymbol{k}$-point grid which is spanned over the 
reciprocal space, i.e. a sampling of the IBZ. This is very important because all quantities, e.g. energy and the electronic density, are computed at those points and summed up, which is computationally less demanding than integration over the whole zone. The IBZ is the reciprocal counterpart of the unit cell, i.e. the smallest unit which build up the whole crystal by translations along the lattice vectors. The mesh of points can either be built up by hand, i.e. writing each point and its weight in the file, or automatically by using schemes to construct sets of special $\boldsymbol{k}$-points like the one developed by Monkhorst and Pack [113]. When using an automatically generated grid one has to define the number of points in $x$-, $y$ - and $z$-direction and the origin of the grid, which can be either the center of the IBZ, or the $\Gamma$-point, the orgin of the IBZ. The number of $\boldsymbol{k}$-points strongly influences the computed energy and computational effectiveness, so it is important to find the optimal value for it from convergence tests. So, as a large lattice vector in real space leads to a small reciprocal vector, large simulation cells need a smaller number of $\boldsymbol{k}$-points to reach convergence. Therefore, when calculating the energy of a system like surfaces, it is sufficient to use a single $\boldsymbol{k}$-point in the direction perpendicular to the surface plane because of the large distance between the images of the simulation cell in this direction.

K-Points \# comment line

0 \# number of $\mathrm{K}$-Points if 0 automatic generation of $\mathrm{k}$-points

Gamma \# Generation including the Gamma point

202020 \# k-points in $\mathrm{x}^{-}, \mathrm{y}^{-}$, and $\mathrm{z}_{\text {-directions }}$

000 no shift in all three directions

Fig. 2: Exemplary KPOINTS-file for an Au bulk calculation.

\subsubsection{POSCAR-file}

The third file necessary to start a calculation is the POSCAR for which an example is shown in Fig. 3. This file contains all geometry information of the considered system, starting with a scaling factor, e.g. the lattice constant of the system, which is followed by the three lattice vectors defining the simulation cell. After that the elements and their number are defined. Next one can define in which coordinates the atomic positions are given. Here, one can choose between Cartesian or Direct coordinates, the latter being the internal coordinates based on the cell vectors. The next optional key word "Selective Dynamics" controls if coordinates of atoms are moved or kept fixed in a relaxation or simulation. After this the position of each atom is written line after line, whereby the triplet of numbers defining the vector is followed by a triplet of logical or Boolean flags 
"T" or "F" when "Selective Dynamics" is used. In case of AIMD simulations the initial velocity of each atom should be defined in $\AA \mathrm{ps}^{-1}$. In case of the CONTCAR-file, a file to continue a previous job, which has the same format as the POSCAR under the velocities the predictor-corrector coordinates of every atom is written.

$\mathrm{Au}(\mathrm{fcc})$ bulk \#comment line

4.20 \# Scaling-Factor(=lattice constant for metals)

0.00 .50 .5 \# 1. lattice vector

$0.50 .00 .5 \# 2$. lattice vector

$0.50 .50 .0 \# 3$. lattice vector

1 \# number of atoms in the system

Selective dynamics use of selective dynamics

Direct \# Direct (fractional) or Cartesian coordinates

$0.00 .00 .0 \mathrm{FFF} \#$ position of atoms with fixed coordinates

Fig. 3: Exemplary POSCAR-file for an Au bulk calculation.

\subsubsection{POTCAR-file}

The last file needed to start a VASP calculation is the POTCAR which contains the ultrasoft pseudo potential (PP) [114] or projector augmented-wave (PAW) [115] basis sets for each species used in the computation in the same order as written in the corresponding POSCAR-file. At the beginning of the POTCAR-file some parameters for the species are defined, e.g. the number of valence electrons (ZVAL) or their atomic mass (POMASS), but also the value for $E_{\text {cut }}$ (ENMAX) for the species which is used if this tag is not specified in INCAR. After this control section the actual PPs or PAWs and their "all-electron" part, as well as the charge deficit (augmented charges), are defined. I address PPs and PAWs shortly in the following.

Both methods overcome the following problem: plane waves do not describe the oneelectron wave function well when it comes close to the nucleus. In this case the wave function changes its shape drastically, i.e. due to orthogonality. With plane waves this change can be described by expanding the basis set and therefore increase the computational effort. Both methods make use of the frozen-core approximation. This means that all electrons within the inner shells are kept in their minimum configuration and their corresponding energy is included in the constructed potentials. So just valence electrons are considered in calculations using either PPs or PAWs. Within the PPapproach a pseudo-wave function is used which has the same shape as the one-electron wave function outside a certain cut-off radius but differs from the true wave function when it comes to the nucleus. The PP gives the same energy when applied to the pseudo 
wave function as when the true Hamiltonian is applied to the corresponding wave function. Especially efficient in calculations are the ultra-soft PPs which were developed by Vanderbilt [114]. The ultra-soft PPs relax the norm-conserving condition of the earlier developed norm-conserving PPs [116], by introducing charge deficits. This has allowed the construction of PPs for the first and second row elements for the first time. Instead of defining a PP, the PAW-method defines a plane wave over the whole space. To account for the differences between the plane wave and the correct wave function close to the nuclei, the plane wave is augmented by projector functions in the regions around the nuclei. As the wave function is defined over the whole space the PAW method can be used to calculate energy and other properties as the electron-density within "allelectron" accuracy. But the latter term is misleading because it refers to the one-electron orbital which is used to solve the radial Schrödinger equation. Due to the representation of the one-center expansions on radial grids the PAW-method is computationally more efficient than even ultra-soft PPs. The first one can be seen as a generalization of the second one and builds a bridge between the augmented plane waves methods [117, 118]. As shown by Joubert and Kresse [119] both methods described above can be transformed into each other. Therefore, both methods should lead to very similar results, but as the PAW-method is more efficient, I used the corresponding POTCAR-files within VASP for all calculations in this thesis.

\subsection{Fritz Haber Institute ab initio molecular simulations}

The FHI-aims code is developed at the Fritz-Haber institute in Berlin. This program can solve the electronic structure problem from first-principles within DFT based on LDA up to hybrid functionals, Hartree-Fock or perturbation theory, like second-order MøllerPlesset theory. The solution of the structure problem can then be used to calculate gradients of the total energy, i.e. the forces on every atom or the second derivative of the energy with respect to positions to get vibrational frequencies in the harmonic approximation. The computation of the forces enables geometry optimization and AIMD simulations as well, but it can also be used to look for the TS by searching for the saddle points on the PES. In contrast to VASP, FHI-aims utilizes numeric atomcentered orbital (NAO) basis functions to describe the wave function of each electron, which allows a true all-electron treatment of the considered system. The functions $\psi_{i}(\boldsymbol{r})$ consist of two parts: a complex spherical harmonic $Y_{l m}(\Omega)$ depending on angular momentum $\Omega$, hence the shape and the orientation of the function given by $l$ and $m$; as well as a real-valued term $\frac{u_{i}(r)}{r}$ which takes care of the radial shape of the orbital. To ensure efficient calculations and full flexibility the numeric values of the latter are 
tabulated, e.g. on a logarithmic grid, which give the orbitals their name. NAOs have two features which distinguish them from other basis functions used in all-electron DFT. Firstly, using the radial functions of occupied free-atom orbitals in the basis, the allelectron orbital shape and nodes close to the nuclei, i.e. under the nuclear $\frac{Z}{r}$-potential, are almost exact in bonded structure as well. Secondly, each radial function $u_{i}(r)$ can be strictly localized inside a given radius by setting $u_{i}(r)$ to zero outside the radius. The latter is particularly useful because different spatial regions of large systems are strictly separated from each other, i.e. basis functions from these regions do not overlap with each other. This enables a very efficient (nearly $\mathrm{O}(\mathrm{N})$ ) scaling with the system size $\mathrm{N}$ of the necessary grid-based computations. In addition the localized nature of NAOs allows us to impose constraints on the effective occupation numbers of certain regions in space and/or on different spin channels, e.g. allowing approximate enforcement of a certain spin state of a molecule in a given environment. This last point was at first the most important reason to choose FHI-aims as a second DFT code to do calculations within the framework of this thesis. Since all electrons are treated explicitly within FHI-aims one needs to take into account relativistic effects at least for heavier elements with an atomic number above 19. For these elements the Coulomb repulsion near the nucleus experienced by the electrons is so strong that their velocity comes in the range of that of the speed of light. Due to this the SE needs to be replaced by the Dirac equation [120] to describe the situation. Unfortunately using the Dirac equation is computationally very demanding. To avoid this and getting a manageable kinetic energy operator two general approaches exist. One separates core and valence states, e.g. as done in the PP $[114,121]$ or muffin-tin based [117] methods, and the other is to approximate the scalar-relativistic Hamiltonian derived from the Dirac equation to correct the obtained approximated energy in a perturbative way. The second variant is used in FHI-aims in the zeroth order regular approximation as introduced by VAN LENTHE and co-workers [122].

There are only two files needed to start a FHI-aims calculation: the control . in and the geometry . in file. The former takes into account all information about the calculation, that means what to do and how to do the actual computation, the latter contains all structural information about the considered system.

\subsection{1 geometry.in-file}

The geometry. in, as shown in Fig. 4, contains the atomic positions and if required the shape of the simulation cell in periodic calculations. This shape is defined by defining the corresponding lattice vectors with the keyword (lattice_vector). An atom position is defined by using keyword at om before the coordinate triple of numbers and followed 
by the element symbol of the species. In case of a spin-polarized calculation one must also define the initial spin of at least one species with the initial_magmom keyword under the corresponding atom in this file. Relaxation constraints on an atom can be imposed by writing relaxation_constraint and the desired coordinate or ".true. ", for all coordinates, under the atom. At this site the velocity of the atom is written with velocity and a triple of numbers in the case of a molecular dynamics calculation. In contrast to VASP the order of the species in the geometry . in file must not respect the order of basis sets setting in the control. in.

$\begin{array}{lcccc}\text { \# Ag(111) vaccum }=20.0 \text { Ang } & \text { layer: } 4 & \text { latconstant: } 4.200 \\ \text { lattice_vector } & 8.90954590 & 0.0000 & 0.0000 \\ \text { lattice_vector } & -4.45477295 & 7.71589279 & 0.0000 \\ \text { lattice_vector } \quad 0.00000000 & 0.0000 & 27.2746143 \\ \text { \# velocities in Ang/ps } & & & \\ \text { atom } & -2.269799574 & 3.271372199 & 5.540365509 \mathrm{C} \\ \text { velocity } & 3.81364044 & 52.74274071 & -23.62475973 \\ \text { atom } & -2.195576650 & 4.297877043 & 5.469725868 & 0 \\ \text { velocity } & -2.86023033 & -39.55705553 & -17.27308535\end{array}$

Fig. 4: Exemplary geometry . in-file for a trajectory of $\operatorname{CO}(v=17)$ in vacuum.

\subsection{2 control. in-file}

control . in, as presented in Fig. 5, contains all keywords regarding the physical description of the system: from the XC functional (xc) over the treatment of relativistic effects and the spin, to the convergence criteria for the electronic (sc_convergence_etot, sc_accuracy_eev, sc_accuracy_forces) and geometry optimization (relax_geometry). The electronic occupation can be controlled by smearing functions like Gaussian or those from MethfESSEL and PAXTON with an appropriate $\sigma$. Further the mixing of the new and old electron densities, i.e. squared wave functions as well as their spin counterpart in the self-consistent field (scf)-cycle can be adjusted. Therefore, different types of mixers are available like Broyden [123], Pulay [112] and Kerker [124], and the latter is recommended for periodic systems. In case of AIMD the desired ensemble, $N V E$ or $N V T$, the length and time step can be defined as well as an option to write out a restart file to continue a simulation. Periodic systems need in addition to lattice vectors in geometry. in the definition of a $\boldsymbol{k}$-point grid in this file. Further keywords can be set to request the output of, e.g. electron or spin density in the cube format.

The control. in has to contain the basis set functions of each species, for which three different basis sets were defined in advance. A small basis set containing just a minimal 


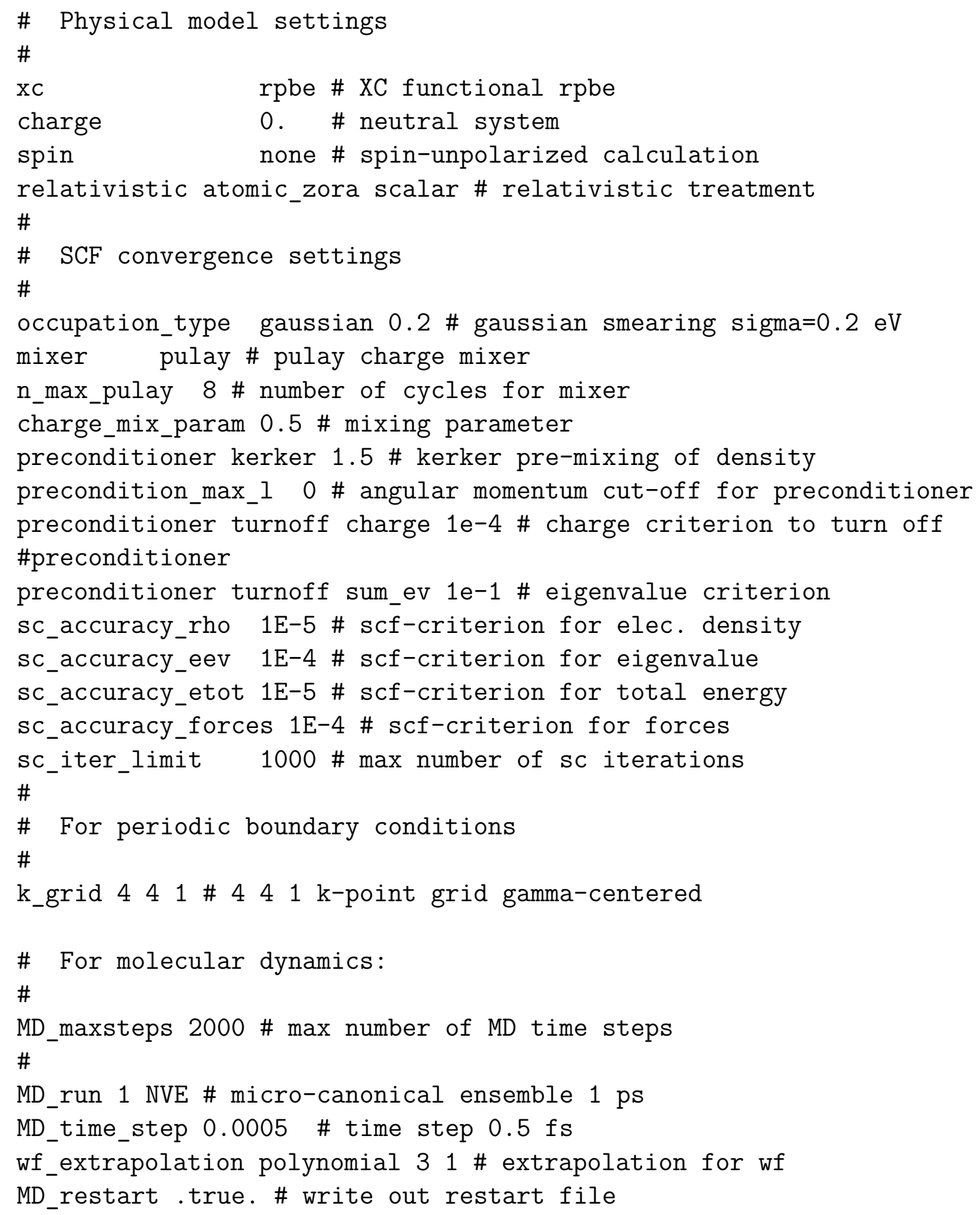

Fig. 5: Exemplary control . in-file for a trajectory of $\mathrm{CO}(\nu=17)$ in vacuum (without the basis set settings). 
basis, i.e. just the occupied orbitals of the species, and few additional orbitals is called a light setting mostly for testing purposes. Basis sets of this type give not so well converged results with respect to absolute energies but give similar results to larger basis sets with respect to energy differences. The second pre-defined basis set settings, called tight, give converged results within the meV-range with respect to absolute energies. These sets should be used to check results obtained using the light settings. As third default there are really-tight basis sets containing the same number of basis sets as the tight ones but other keywords, like basis_dep_cutoff , are set in that way to approach the converged basis size limit. Therefore, really-tight-settings should only used for special tests to verify results obtained with tight, as the former settings are strongly overconverged.

\subsubsection{MEP calculations in FHI-aims}

For calculation of MEPs, see section 3.5, with FHI-aims it is recommended to use the aimsChain utility [105]. This utility, written in Python, controls the search for a TS by using a chain. in file, additionally to the control. in. In the former the method used for the search, the number of images and the convergence criterion for the forces acting on the whole path as well as on the climbing image should defined. With the two files containing the initial and final configurations one can start the search by starting the python tool. The control. in must contain the keyword to evaluate the forces on every atom. It carries out a separate FHI-aims calculation for each image and moves then the images in such a way that the forces acting on every image are minimized until the convergence criterion is reached.

\subsection{Phonopy}

Phonopy is an open-source software package to calculate phonon spectra using the finite differences approach in the (quasi-)harmonic approximation. It is written in python and has been developed by Atsushi Togo. The following description is strongly based on [106]. The program does not just enable the calculation of density of states (DOS)s or their projection on single bands or orbitals projected density of states (PDOS) by analyzing the band structure of the phonons, i.e. vibrations in solid states but also calculate thermal properties as heat capacity, free energy and entropy. Other quantities as mean square displacements and reducible representations of the normal modes and Grüneisen parameters can be calculated too. Interfaces for a variety of different DFT codes are available, e.g. VASP, FHI-aims but also WIEN2k, ABINT, Quantum ESPRESSO or TURBOMOLE. 
To start a computation with Phonopy to obtain the DOS of a desired structure one has to define the following input files: a setting file, that means a control file, for Phonopy where all settings are defined; a structure file which contains the configuration in the appropriate format of the DFT program used to calculate the forces, and finally a FORCE_CONSTANTS file where the force constants from each coordinate displacement and the corresponding super cell are written to. The following tags need to be defined in the setting file: first all things regarding the super cell (DIM-tag), the displacement of the individual atoms and the kind of the species and, most important, the file contains the unit cell. The former can be defined in two ways either by writing three integers after the DIM-tag, thus creating these numbers of original unit cells in each direction of space, or writing nine integers to define the supercell matrix which is multiplied with the unit cell to create the supercell. With this Phonopy can generate the files, which contain the structure with one displaced atom. Those structures can be used to calculate the forces with an appropriate DFT code before doing post-processing with Phonopy.

For the force constants one has to specify if they should be read from the FORCE_CONSTANTS input file or written to it by calculating them from the FORCE_SET file which comprises the forces acting on every atom in a supercell. These are obtained from the output file of the respective force calculator, i.e. the used DFT code. In case of VASP the forces of every displaced super cell are read from the vasprun.xml file. Therefore, one has to do a calculation of every displaced super cell configuration, maybe generated via the displacement settings by Phonopy, with an appropriate DFT program in advance. The force constants are obtained by using the finite displacement method. Thus a fitting procedure is used to fit all force constant elements between a primitive cell and the supercell to symmetry expanded forces of atoms in the supercell [107]. Of course there are also settings to request and control the Phonopy output, e.g. a DOS needs the specification of a mesh (MESH), similar to a $\boldsymbol{k}$-point grid but finer, and its range (DOS_RANGE) must be defined by a number of points and a proper spacing between those points; in addition for the PDOS one needs to define the bands on which the states should be projected. Phonopy comes with a plotting utility, thus uses matlibplot to plot the results, as well as generating files to animate resulting normal modes in different formats.

Also for the calculation of the vibrational frequencies an external tool exists which is based on the Phonopy code described in the next section. For such calculations FHI-aims is only used as a tool to calculate the forces acting on every atom. The tool does all the necessary steps to calculate the vibrational frequencies within the finite distances approach, thus in the harmonic approximation. So it generates all required geometry . in files by respectively displacing all atoms twice in $x$-, $y$ - and $z$-direction 
of a provided structure. After all calculations are done the utility uses the forces and displacements to compute all frequencies and normal modes, thus the eigenvalues and eigenvectors of the system.

\subsection{Bader analysis}

The Bader charge analysis program, developed by the Henkelman group [125], analyzes electron density files written either in the cube format or in the CHGCAR-format of VASP. The program, based on Bader Quantum Theory of Atoms in Molecules [109], looks for maxima within the electron density because in most cases these maxima are close to the nuclei as nuclei attract the electron density. From a maximum it looks for regions where the density gradient goes asymptotically to zero [54]. Such regions are represented by so called zero-flux surfaces which form a polyhedron around a certain maximum of the electron density. Now the maximum is assigned to the closest atom and all density within the polyhedron than is allocated to this atom. Integrating the density over the volume of the polyhedron then gives the number of electrons and so the electric charge on this atom. In both file formats the density is written on a uniform grid. This grid needs to be very dense to get accurate and reliable results for the total number of electrons $N$, especially in case of cube-file which contains an all-electron density. The reason for this is that the densities of the electrons in the inner shells (the non-valence shells) of the atoms are in a smaller volume than the sparser density of the valence electrons. In particular, elements with a high atomic number such as noble metals have a very high electron density in the region of the inner shells. Therefore, this region has to be sampled with many grid points so that all existing density is taken into account in the Bader analysis.

For CHGCAR files generated by VASP the situation is different. Since VASP is based on PP the CHGCAR file just contains the valence electron density. Finding the correct maxima in this electron density can be problematic for the Bader analysis program. Hence, the partitioning of the density to the polyhedrons is incorrect. To get more reliable results from the analysis it is possible to use a reference electron density which is compared to the actual analyzed density. In that way it is possible to get a quasi all-electron density from a VASP calculation. Therefore, the LAECHG-tag has to be set to ". TRUE. " in the INCAR file. Thus, VASP reconstructs the electron density of the core states or region with its nodal features as well as the proto-atomic valence density from the PPs or PAWs. The latter density is the overlapping atomic charge density which is not necessary for the reference file. At the end of the calculation, hence when self-consistency is reached, the corresponding valence density is written out. The sum of valence and core density 
yields the reference density usable for the Bader analysis. This sum is not equivalent to an all-electron density obtained from a FHI-aims calculations, because it is based on the PP wave function which differs from the all-electron wave function within the region of the core electrons.

In subsection 7.4.4, the Bader program is tested with different cube and CHGCAR-files and investigated the difference between a PP and an all-electron code. For VASP I also investigated the influence of a reference file on the results of the charge analysis.

\subsection{Finding minimum energy pathways}

Finding MEPs and TSs is of great interest for a variety of systems and hence is a very active field of research over the last years and decades. Knowing the configuration and energy of the TS is important to predict the progress of a reaction, e.g. which kind of motion promotes a reaction according to Polanyi's rule. With the energy of TS one can determine the reaction rate which is essential for the kinetic modeling of chemical processes. Therefore, methods to obtain such pathways have been developed [126-130]. In principle, there are two different approaches to find a TS depending on the knowledge of the PES. On one hand one can use an approach using only one configuration. On the other hand an interpolation method is possible where two configurations are used to determine the pathway. The first one needs a good estimation of the TS configuration, and then the intrinsic reaction coordinate must be followed to determine the MEP and the configuration of the reactant and the product. Methods which are based on this idea like coordinate driving, linear and quadratic synchronous transit, and sphere optimization are not further discussed because they were not used in the following.

When using two configurations to find the MEP the obvious choices for them are the minimum structures of the reactants and the product. Hence, the TS of the reaction must be somewhere between both configurations. For this method there exist generally two approaches to tackle the problem: one approach uses one or two trial structures which are moved between the two initial structures to find the TS. The second approach samples the configuration space between reactant and product with multiple numbers of trial configurations, also called images, to find the TS and MEP, hence the whole pathway, as well. This set of configurations has then to be optimized with respect to a minimized total energy. Methods using this approach are often called chain-of-states methods. Two frequently used methods are the nudged elastic band (NEB) and string method [127, 129]. Since both methods are used in this work their principles and differences will be described in the following. 
NEB and string method have in common that all images along the pathway are connected via a spring constant. So the energy expression which has to be minimized consists not only of the sum of the energies of all images $M$ between initial and final state $\boldsymbol{R}$ and $\boldsymbol{P}$ but additionally of a term which connects two adjacent configurations via the spring constant $k$, ensuring an equal distribution along the path:

$$
E_{\mathrm{NEB}}\left(\boldsymbol{R}, \boldsymbol{x}_{1}, \boldsymbol{x}_{2}, \ldots, P\right)=\sum_{i=1}^{M} E\left(\boldsymbol{x}_{i}\right)+\sum_{i=1}^{M-1} \frac{1}{2} k\left(\boldsymbol{x}_{i+1}-\boldsymbol{x}_{i}\right)^{2} .
$$

The resulting minimization depends strongly on the spring constant. If it is too large then the pathway tends to cut corners, whereas a smaller force constant leads to a sliding of the images towards the initial (minima) configurations. This can be avoided by using more images to sample the pathway. This leads to problems in the optimization procedure, e.g. more computational effort due to more images or convergence of the calculations. Instead of this a more feasible solution is to nudge the elastic band, that is using only the component of the spring force parallel to the tangent of the path and the component of the force, due to the potential energy of the configuration, perpendicular to the path when doing the optimization of $E_{\mathrm{NEB}}$. The MEP obtained in that way does not include the saddle point, but it can be found with the Climbing Image extension of the NEB. Here one of the images, usually that with the highest energy, is allowed to move along the elastic band so that the correct saddle point of the PES is obtained.

With the NEB method the images along the pathway are redistributed after each optimization cycle based on projected (perpendicular) force [131]. The string method is an alternative to that, where the optimization is improved by using all components of the force instead of the force projections [129]. It leads to a numerically more stable implementation, because to calculate the force projection on the tangent along the path is computationally more expensive.

Another difference between NEB and string method is that the former uses artificial spring constants to prevent the images falling towards the minima, and in the latter it is done by enforcing a special parametrization which can be described as interpolation by filling up the space created along the path due to the potential force. Alternatively, the string method can be viewed as an in-extensible limit of the NEB method, where $k \rightarrow \infty$, which explains the name of this method. 


\section{Build up the system and analyze the results}

In this chapter I will describe first how I construct the slab representing the metal surface in the DFT calculations. Secondly, I will cover the issue of the preparation of the system used in the AIMD simulations. This addresses slab preparation as well as the determination of quantities describing the molecule at the start of a simulation. Finally, I will cover the procedure how I analyzed the outcome of the computed trajectories and how I got to the results shown in the next chapter.

\subsection{Building up the System}

To do simulations for scattering experiments of $\mathrm{CO}$ and NO from metal surfaces a model to represent the surface is needed. A real metal surface as used in the scattering experiments is a small polished crystal which has a size of a few mms and consists of a very large number $\left(\sim 10^{18}\right)$ of atoms. But the atoms are all in periodical order; modeling such a crystal is possible knowing the form of the unit cell, i.e. the smallest building block of the crystal.

\subsection{1 fcc-lattice geometry}

Since all metals used in this work crystallize in the face-centered cubic $(f c c)$ structure the unit cell should be described briefly. As the name implies the framework of this structure is a cube, with an edge length of $a$, where on each corner as well as at the center of each of the cubic faces sits an atom. From this picture, as shown in Fig. 6, one can look for a smaller building block which can form the whole crystal, thus the unit cell. 


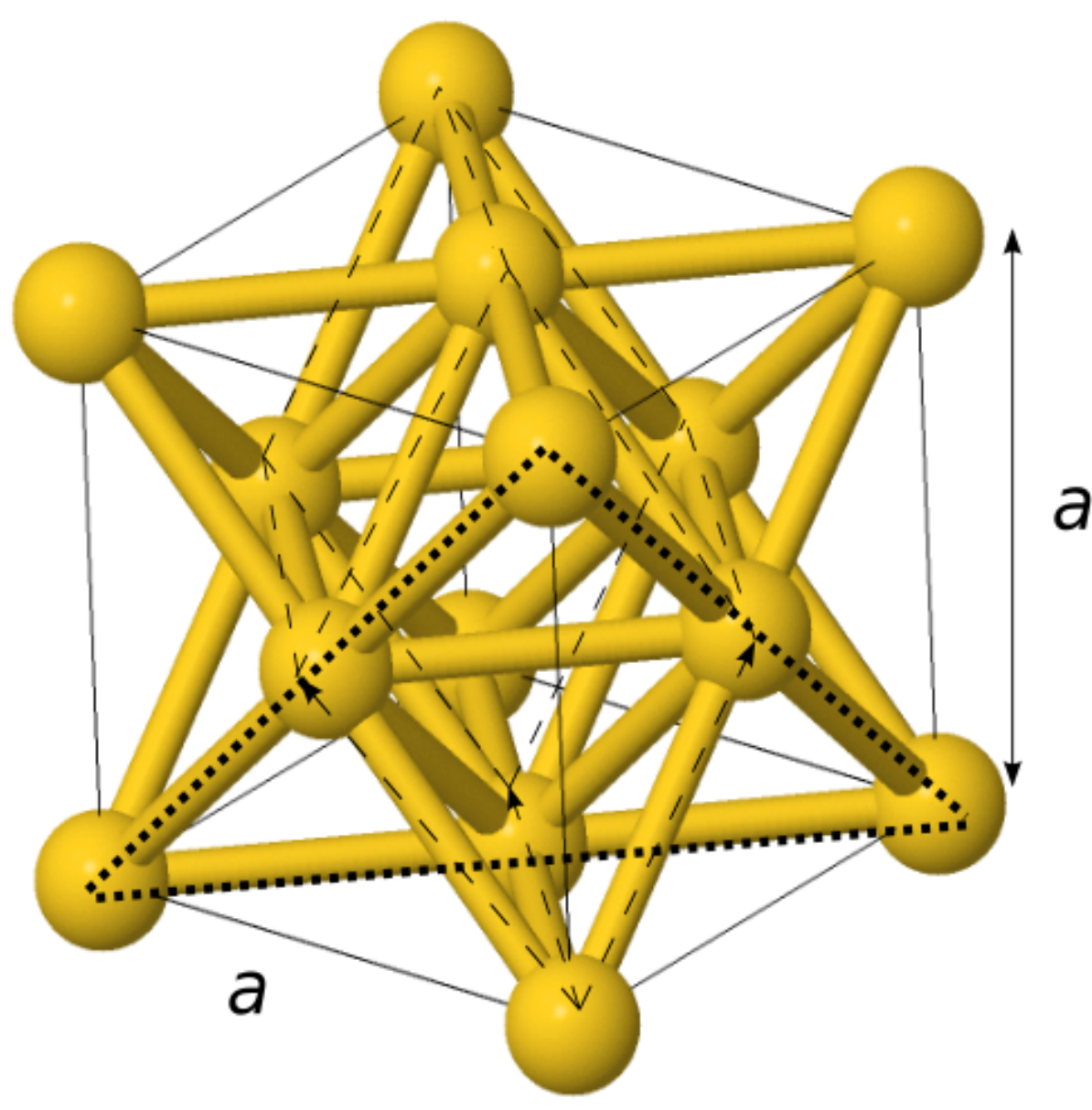

Fig. 6: A crystal in its $f c c$ metal structure. The cube is indicated by the thin black solid lines which have a length $a$. The unit cell and its lattice vectors are indicated by thin dashed lines and arrows, respectively. The triangle shown by thick dotted lines represents one possible cut to create a (111)surface.

The $f c c$ cell is described by the following lattice vectors:

$$
\begin{aligned}
& \boldsymbol{a}_{\mathrm{fcc}}=(0.5,0.5,0.0) a, \\
& \boldsymbol{b}_{\mathrm{fcc}}=(0.0,0.5,0.5) a, \\
& \boldsymbol{c}_{\mathrm{fcc}}=(0.5,0.0,0.5) a .
\end{aligned}
$$

A possible unit cell within the $f c c$ structure is indicated by the thin dashed black lines in Fig. 6.

The surface is produced via cutting the crystal in a certain way, so that the normal of the (111) surface is along the space diagonal of the $f c c$-cube. The cut forming a triangle is 
represented by thick dotted lines in Fig. 6 . This surface plane and the distance between planes are described by the following vectors:

$$
\begin{aligned}
& \boldsymbol{a}_{111}=\left(\frac{1}{\sqrt{2}}, 0,0\right) a, \\
& \boldsymbol{b}_{111}=\left(\frac{-1}{2 \sqrt{2}}, \sqrt{\frac{3}{8}}, 0\right) a, \\
& \boldsymbol{c}_{111}=\left(0,0, \frac{1}{\sqrt{3}}\right) a .
\end{aligned}
$$

The last vector $\boldsymbol{c}_{111}$ is not a lattice translation vector but just its $z$-component marking the direction in which the periodicity of the crystal is broken.

These vectors can be derived from the $f c c$-bulk structure by considering the following: The triple of numbers (111) are Miller indices, in general $(h k l)$, specify how the bulk is cut and the reciprocal lattice. The (111)-surface is presented in Fig. 6 by the thick dotted lines. Since each side of this equilateral triangle is along a diagonal of the face of the cube one can easily find two lattice vectors describing the (111)-plane. The first vector needs to translate an atom along one of the marked diagonals to another one, whereas the second vector has to move the atom on the another diagonal. With this the first vector points just from one to a neighboring atom on the same diagonal as defined by $\boldsymbol{a}_{111}$, and the components of $\boldsymbol{b}_{111}$ can be found by using trigonometrical arguments and the angle between the two vectors, which is $120^{\circ}$.

The third vector $\boldsymbol{c}_{111}$ is perpendicular to the plane spanned by the two other vectors and so its length gives the distance to the next (111)-plane. Since the (111)-plane is perpendicular to the space diagonal of the cube with a length of $\sqrt{3} a$, and there are 3 (111)-planes crossing this diagonal in a $f c c$ cube (see Fig. 6), the distance between two adjacent layers in this direction is a third of the length of the diagonal of the cube, $\frac{1}{\sqrt{3}} a$. Since the (111)-surface is generated from the $f c c$ structure it also inherits the $A B C A \ldots$ structure for its layers. It means that the $x, y$-positions of layer origin in the $1^{\text {st }}, 2^{\text {nd }}$ and $3^{\text {rd }}$ layers are shifted relative to each other, while they are the same in every $4^{\text {th }}$ layer. With geometrical arguments it is possible to derive the $x$ - and $y$-coordinates of the $2^{\text {nd }}$ and $3^{\text {rd }}$ layers with respect to both coordinates in the first one. Given the origin of the first layer has the $x$-, $y$ - and $z$-coordinates equal to zero, the $2^{\text {nd }}$ layer has a shift equal to $\left(\frac{1}{3}, \frac{2}{3}, 1\right)$ in fractional coordinates, and the $3^{\text {rd }}$ layer is shifted to $\left(\frac{2}{3}, \frac{1}{3}, 2\right)$. To get Cartesian coordinates from these fractional ones, one has to multiply the first value of the triplet with the vector $\boldsymbol{a}_{111}$, the second with $\boldsymbol{b}_{111}$ and the third with $\boldsymbol{c}_{111}$ as defined above. 


\subsubsection{Preparing the simulation cell}

Since the fcc unit cell and the cut of the (111)-surface have been described I will now shortly describe how the equilibrium lattice constant is determined which is needed to build up the simulation cell. It is used to model the surface in vacuum and eventually a molecule above it.

\subsubsection{Equilibrium lattice constant of the $f c c$ metal}

The equilibrium lattice constant $a$ is determined via calculating the cohesive energy of the bulk for different values of lattice constant and taking the one which corresponds to the minimum energy. The convergence of results was checked against $\boldsymbol{k}$-point grid size and smearing function and its width $\sigma$ (see 2.3). The result of such a calculation is presented in Fig. 7 and the input files (except the POTCAR) are shown in Figs. 1 - 3. To simulate the bulk (crystal) structure of the metal just one atom was used which is repeated along the lattice vectors of the fcc unit cell, as given in (36), by employing periodic boundary conditions (pbc).

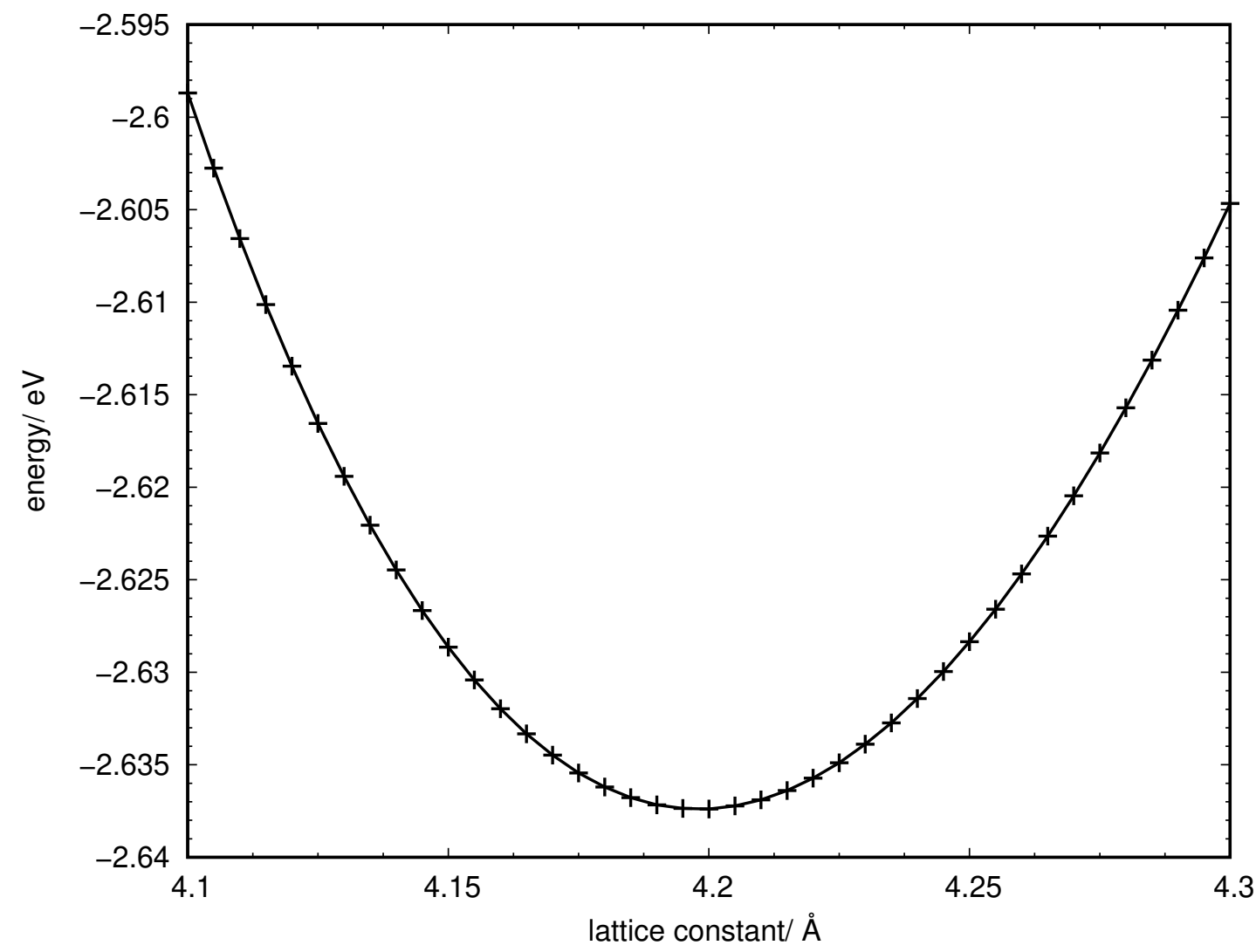

Fig. 7: Cohesive energy of a Au bulk vs. the lattice constant. Calculated with VASP using the RPBE functional and a $20 \times 20 \times 20 k$-point grid. 


\subsubsection{2 (111) surface}

To build up different layers with a (111)-facet we can directly use the lattice vectors from Eq. (37) and the (shifted) origin, of each layer, of which the $x$-and $y$-positions can also be found in section 4.1.1. This means every layer is constructed by translating the atom at the origin along $\boldsymbol{a}_{111}$ and $\boldsymbol{b}_{111}$ as well as adding both vectors together; thus the two vectors describe the primitive cell of the surface. Its size is given by integers $n$ and $m$ by which every vector is translated; therefore the cell is characterized as $p(n \times m)$. The $z$-direction of every layer is given by moving along $\boldsymbol{c}_{111}$ which can then be described as $\mathrm{p}(\mathrm{n} \times \mathrm{m})$ slab with $i$ layers.

To build up the simulation cell - also referred to as super cell - in which the simulations take place three lattice vectors need to be defined. The cell is repeated by these vectors in all directions in space according to pbc. So the in-plane vectors are $n \boldsymbol{a}_{111}$ and $m \boldsymbol{b}_{111}$. The size of the cell in the $z$-direction is defined by the number of layers and the width of the vacuum layer, which is necessary to avoid the so-called image interaction, which is the interaction between atoms in different cells. This is illustrated in Fig. 8b where a super cell of a $\mathrm{p}(3 \times 3)$ slab with 4 layers and its images in the $x-, y$ - and $z$-directions are shown. In practice the number of layers and the width of the vacuum layer should be checked with respect to a converged energy. For this the numbers of layers should be at least three in the case of $f c c$-structure to account for the $A B C$ series of the layers.

To differ between the three layers one refers to the uppermost layer (A) as top, the layer beneath (B) as hexagonal-closed packed ( $h c p$ ) and the third one (C) as fcc. This comes from the fact that the hexagonal close packed structure has just two different layers whereas the cubic close-packed structure, the $f c c$ structure comes along with three distinguishable layers. So the layers as well as atoms within the corresponding layers are named after these abbreviations. They are also used to refer to adsorption sites on the surface. In case of these sites top refers to the situation when the molecule sits above an atom in the top layer, bri(dge) is situated between atoms in the top layer, so bridging two atoms, whereas $f c c$ and $h c p$ refer to sites above atoms within those layers. In Fig. 8a the different layers of the (111) surface in the $f c c$ structure as well as the mentioned adsorption sites are shown.

The two in-plane cell vectors have to be large enough so that lateral image interactions between a molecule and its periodically repeated images are minimized. But with longer vectors the number of atoms in the simulation cell increases and therefore the computational effort for the calculations increases. So this limits the choice of $n, m$ for the $p(n \times m)$-slab to small integers.

The super cell shown in Fig. $8 \mathrm{~b}$ is mostly used for the calculations and simulations to represent a model for the (111)-transition metal surfaces of Au, Ag, Cu and Pt. Since this 


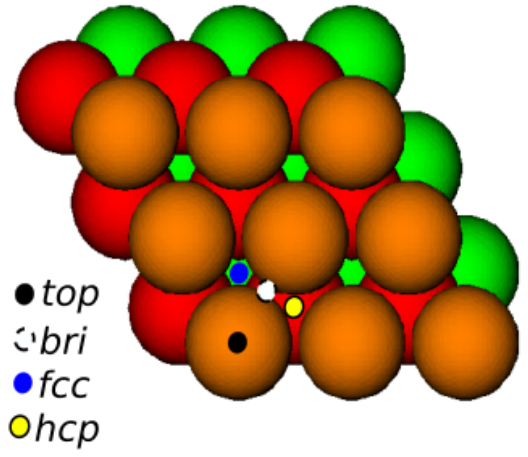

(a) A p (3×3) slab of a (111) surface from top view. The atoms of the three different layers are represented by orange, red and green balls for the top, hcp, and fcc layer, respectively. Further labeled are 4 adsorption sites on the surface.
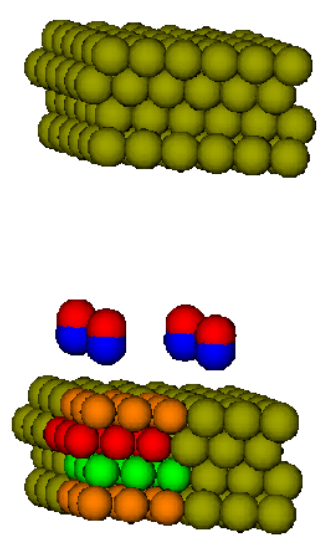

(b) A simulation or super cell of an $\mathrm{Au}(111) \mathrm{p}(3 \times 3)$ slab with 4 layers and a NO molecule (blue and red spheres) from side view. Shown is the original cell with orange, red and green spheres. Images of the surface in the $x$-, $y$ - and $z$-directions represented by olive spheres and the molecule in $x$-, $y$-directions are shown. The white space in $z$-direction is the vacuum distance.

Fig. 8: A p (3×3) cell of a (111) surface from top view and a simulation cell using the former with 4 layers and a NO molecule above. The periodicity is indicated with adjacent images shown in olive.

choice of the super cell minimizes lateral interactions with the periodic images by using a minimum number of atoms to keep the computational effort feasible. 


\subsection{Procedure to prepare AIMD simulations}

To perform AIMD simulations corresponding to the desired surface temperature it is necessary to define the initial positions and velocities of all atoms in agreement with the respective thermal distributions. For this purpose an AIMD simulation with the slab representing the surface is carried out which is referred to as slab equilibration. Here, the atoms are set to their equilibrium positions as determined by the corresponding lattice constant $a$ and the atom velocities are initialized by a Maxwell-Boltzmann distribution, which is characterized by the temperature. To avoid a drift of the slab through space its bottom layer is kept fixed, i.e. the atoms are at their equilibrium positions during the simulation. Thus the atoms in this layer are not taking part in the equilibration. The temperature is related to the averaged kinetic energy of the system as

$$
\left\langle E_{\text {kin }}\right\rangle=\frac{3}{2} N_{\mathrm{p}} \mathrm{k}_{\mathrm{B}} T
$$

where $N_{\mathrm{p}}$ is the number of moving particles in the system, $k_{\mathrm{B}}$ is the Boltzmann constant and $T$ is the temperature. The virial theorem of an harmonic lattice states that $\left\langle E_{\text {kin }}\right\rangle$ is equal to $\langle V\rangle$, the averaged potential energy, but this is at its minimum, because all atoms initially are at their equilibrium positions. In the equilibration the kinetic energy is redistributed into potential energy because the atoms are moving away from their equilibrium positions and so the averaged kinetic energy decreases as given by (38). Therefore, the value of the temperature has to be two times higher than the desired value. So to have the system in equilibrium at the desired temperature, the above mentioned AIMD simulations are computed as long as the system temperature is only fluctuating around the desired value, i.e. $300 \mathrm{~K}$. When this state is reached the system is supposed to be in equilibrium at a certain temperature. From that the calculated positions and velocities of the atoms are used as initial configurations in phase space to represent a surface at the desired temperature.

\subsubsection{Preparation of the initial state of diatomic molecules}

After the description of the procedure preparing the slab I will move on to the molecule consisting of atoms A and B. To define its initial position I used the following two vectors $\boldsymbol{r}_{\mathrm{cm}}$, the COM position of the molecule and the bond vector between the two atoms 
$\boldsymbol{r}=\boldsymbol{r}_{\mathrm{B}}-\boldsymbol{r}_{\mathrm{A}}$. The former is defined by the Cartesian coordinates $x_{\mathrm{cm}}, y_{\mathrm{cm}}, z_{\mathrm{cm}}$ and the bond vector is given by

$$
\boldsymbol{r}=\left(\begin{array}{c}
r_{x} \\
r_{y} \\
r_{z}
\end{array}\right)=\left(\begin{array}{c}
r \cos (\phi) \sin (\theta) \\
r \sin (\phi) \cos (\theta) \\
r \cos (\theta)
\end{array}\right)
$$

where $r_{x}, r_{y}$ and $r_{z}$ are the $x$-, $y$ - and $z$-coordinates of $\boldsymbol{r}, r$ its length, $\theta$ is the angle between $\boldsymbol{r}$ and the $z$-axis and $\phi$ is the angle between $\boldsymbol{r}$ and the $x$-axis. With the molecular orientation and COM the positions of the atoms can be obtained to

$$
\boldsymbol{r}_{\mathrm{A}}=\boldsymbol{r}_{\mathrm{cm}}-\frac{m_{\mathrm{B}}}{M} \boldsymbol{r} \quad \boldsymbol{r}_{\mathrm{B}}=\boldsymbol{r}_{\mathrm{cm}}-\frac{m_{\mathrm{A}}}{M} \boldsymbol{r},
$$

where $m_{\mathrm{A}}$ and $m_{\mathrm{B}}$ are masses of atoms $\mathrm{A}$ and $\mathrm{B}$, respectively, and $M=m_{\mathrm{A}}+m_{\mathrm{B}}$ the total mass of the molecule. Therefore, the atomic positions can be described by the six parameters: $x_{\mathrm{cm}}, y_{\mathrm{cm}}, z_{\mathrm{cm}}, r, \phi$ and $\theta$.

However, not only the atomic positions $\boldsymbol{r}_{\mathrm{A}}$ and $\boldsymbol{r}_{\mathrm{B}}$ need to be determined but the velocities $\boldsymbol{v}_{\mathrm{A}}$ and $\boldsymbol{v}_{\mathrm{B}}$, too, have to be set initially. The latter can be defined from the initial energetic state in which the molecule is. This is given by the initial experimental conditions, i.e. initial translational energy, rotational and vibrational state, which should be simulated. For this reason, I explain in the following how the molecular energy is distributed over the different dof and how it is related to the atomic velocities.

The energy of the diatomic molecule is given [132]

$$
E_{\mathrm{mol}}=T_{\mathrm{mol}}+R+T_{\mathrm{v}}+U
$$

where $T_{\mathrm{mol}}, R, T_{\mathrm{v}}$ and $U$ are the translational, rotational, vibrational kinetic and potential energy of the molecule, respectively, determined by the following equations

$$
T_{\mathrm{mol}}=\frac{1}{2} M \boldsymbol{v}_{\mathrm{cm}}^{2}, \quad R=\frac{1}{2} \boldsymbol{\omega} \cdot \boldsymbol{I} \cdot \boldsymbol{\omega}=\frac{1}{2} \mu \boldsymbol{v}_{\mathrm{rot}}^{2} \quad \text { and } \quad T_{\mathrm{v}}=\frac{1}{2} \mu \boldsymbol{v}_{\mathrm{vib}}^{2} .
$$

Here $\mu=\frac{m_{\mathrm{A}} m_{\mathrm{B}}}{M}$ is the reduced mass and

$$
\boldsymbol{v}_{\mathrm{cm}}=\frac{m_{A} \boldsymbol{v}_{A}+m_{B} \boldsymbol{v}_{B}}{M}, \quad \boldsymbol{v}_{\mathrm{rot}}=\boldsymbol{\omega} \times \boldsymbol{r} \quad \text { and } \quad \boldsymbol{v}_{\mathrm{vib}}=\boldsymbol{v} \frac{\boldsymbol{r}}{|\boldsymbol{r}|}
$$

are the translational, rotational, and vibrational velocity of the molecule, respectively;

$$
\omega=\frac{\boldsymbol{v} \times \boldsymbol{r}}{\boldsymbol{r}^{2}}
$$


is the angular velocity,

$$
\underline{\underline{I}}=\mu[(\boldsymbol{r} \cdot \boldsymbol{r}) \underline{\underline{E}}-\boldsymbol{r} \otimes \boldsymbol{r}]
$$

is the inertia tensor, where $\underline{E}$ is the unity matrix and $\otimes$ denotes the outer product. Finally, $\boldsymbol{v}=\boldsymbol{v}_{B}-\boldsymbol{v}_{A}$ is the relative velocity of the atoms in the molecule. The potential energy $U$ depends on the bond length $r$, and we need an expression to describe it. To characterize the potential energy I used the Morse potential [133] as given by

$$
U(r)=D_{\mathrm{e}}\left[1-\exp \left(-\alpha_{\mathrm{M}}\left(r-r_{\mathrm{eq}}\right)\right)\right]^{2}
$$

where $D_{\mathrm{e}}, \alpha_{\mathrm{M}}$ and $r_{\mathrm{eq}}$ are the dissociation energy, the stiffness and the equilibrium bond distance, respectively. The choice of this function is based on two considerations: first it is known to characterize diatomic molecules well, thus it gives comparable results to experimentally determined values including dissociation, and second it can be related to the vibrational state $v$. The energy of the Morse oscillator for a vibrational state $v$ is given as

$$
E_{\text {Morse }}(v)=h v_{0}\left(v+\frac{1}{2}\right)-\frac{\left(h v_{0}\left(v+\frac{1}{2}\right)\right)^{2}}{D_{\mathrm{e}}},
$$

with Planck's constant $h$, and $v_{0}=\frac{\alpha_{\mathrm{M}}}{2 \pi} \sqrt{\frac{2 D_{\mathrm{e}}}{\mu}}$. $E_{\text {Morse }}$ gives the total vibrational energy of the molecule; thus it is the sum of $T_{v}$ and $U$. The rotational energy $R$ contribution to $E_{\mathrm{mol}}$ can be described by a model, the rigid rotor, to assign it to corresponding states. But as in experiment the molecules are rotationally cold, i.e. they are in low states. The rotational contribution was set to zero for all simulations carried out in this work. Thus the rotational dofs were not excited in the prepared molecule.

Finally, I will address the issue how we come from these energetic considerations to velocities of both atoms. The total velocity of both atoms are given by

$$
\boldsymbol{v}_{\mathrm{X}}=\boldsymbol{v}_{\mathrm{cm}}+\boldsymbol{v}_{\mathrm{rot}, \mathrm{X}}+\boldsymbol{v}_{\mathrm{vib}, \mathrm{X}}
$$

where $\boldsymbol{v}_{\mathrm{rot}, X}$ and $\boldsymbol{\nu}_{\mathrm{vib}, X}$ are the rotational and vibrational velocities of atom X, which can be either A or B. Both velocities can be calculated with the rotational and vibrational velocity $\boldsymbol{v}_{\text {rot }}$ and $\boldsymbol{v}_{\text {vib }}$ of the molecule (given by (43)) as

$$
\boldsymbol{v}_{\text {rot }, \mathrm{A}}=-\frac{\mu}{m_{\mathrm{A}}} \boldsymbol{v}_{\mathrm{rot}} \quad \boldsymbol{v}_{\mathrm{rot}, \mathrm{B}}=\frac{\mu}{m_{\mathrm{B}}} \boldsymbol{v}_{\mathrm{rot}}
$$

and

$$
\boldsymbol{v}_{\mathrm{vib}, \mathrm{A}}=-\frac{\mu}{m_{\mathrm{A}}} \boldsymbol{v}_{\mathrm{vib}} \quad \boldsymbol{v}_{\mathrm{vib}, \mathrm{B}}=\frac{\mu}{m_{\mathrm{B}}} \boldsymbol{v}_{\mathrm{vib}} .
$$


After the description of the parameters necessary to characterize the initial state of the molecule I will now describe how I determined these parameters.

The choice of $z_{\mathrm{cm}}$, which describes the distance to the slab, is determined by the following considerations: on one hand it must be large enough so that the interaction energy $V_{\text {int }}$ between slab and the projectile is close to zero; on the other hand the molecule has to be as close as possible to the slab, so that the computational effort is minimized. To find a good compromise between both considerations, $V_{\text {int }}$ was determined as a function of $z_{\mathrm{cm}}$ (see section 7.2) and from that a value for $z_{\mathrm{cm}}$ was chosen as $5.5 \AA$.

The parameters $x_{\mathrm{cm}}, y_{\mathrm{cm}}, \theta$ and $\phi$ were determined by using a random number generator which was later on discarded because it does not lead to randomly distributed initial positions and orientations. Instead of random numbers I used a uniform grid to distribute the molecule over the whole area of the simulation cell. This method was then also used for both orientation angles of the molecule.

The last parameter to set is $\boldsymbol{r}$, for which only its length $r$ needs to be determined as its orientation is given by $\theta$ and $\phi . r$ was determined via the Morse function (46) which was defined by the corresponding parameters. They were obtained by calculating the potential energy of the molecule for different bond distances via DFT and fitting these data with the Morse function. In the case of a simulation with CO in the vibrational ground state $v=0, r$ was set to be the equilibrium bond length $r_{\mathrm{eq}}$. For the molecule set in a higher vibrational state I divided the vibrational energy corresponding to it into the kinetic and the potential fractions. For this decomposition a random number was used. From the first part $T_{\mathrm{v}}$ the velocities are determined, and from the second part $U$ the bond length $r$ is obtained. The relation between $T_{\mathrm{v}}$ and the velocities is determined by (42) and (43). Since potential energy can lead to two values for the bond distance a random number generator is used to make the decision of which one is actually chosen. The initial vibrational energy is linked to the corresponding vibrational state by (47). Finally, the COM velocity $\boldsymbol{v}_{\mathrm{cm}}$ vector is described by its length and its orientation. The former is obtained from the experimentally used incidence energy of the molecule which equals to the initial translational energy $T_{\mathrm{mol}}$, and the orientation is given by the angle between the molecular beam and the surface normal $\theta_{\text {in }}$. This was set to $0^{\circ}$ for all simulations which means that $\boldsymbol{v}_{\mathrm{cm}}$ was directly set towards the slab.

With this, the whole framework to initialize AIMD simulations with diatomic molecules in the vibrational ground state as well as in a vibrationally excited state carried out in this thesis is described. 


\subsection{Analysis of the energy transfer from AIMD trajectories}

To understand the energy transfer between a diatomic molecule and the surface it is important to know how the energy is redistributed between the molecular and surface dof. For this purpose, I describe the separation of the total energy of the system into translational, rotational and vibrational contributions.

In order to separate the energy contributions of our system we define the total energy of this system as

$$
E_{\mathrm{tot}}=T_{\mathrm{tot}}+V=E_{\mathrm{mol}}+E_{\mathrm{slab}}+V_{\mathrm{int}},
$$

where $T_{\text {tot }}$ is the kinetic and $V$ the potential energy of the system. Since the system consists of a diatomic molecule and a slab representing the surface, we can express the energy of the system as a sum of the molecular $E_{\mathrm{mol}}$ and slab contributions $E_{\text {slab }}$ as well as the potential interaction energy between projectile and surface $V_{\text {int }}$. Due to energy conservation the total energy $E$ should be constant along a trajectory. The errors in the numerical integration lead to the existence of a small drift and fluctuations in $E$ which provides an accuracy limit for the values extracted from simulations. When the molecule is far away from the surface, $E_{\mathrm{mol}}$ and $E_{\mathrm{slab}}$ are constant, because $V_{\mathrm{int}}=0$. As we want to clearly separate the former two energies from each other it is necessary to know the distance between the COM of the molecule and the surface where they do not interact.

The total potential energy consists of three contributions:

$$
V=U+V_{\text {slab }}+V_{\text {int }}
$$

where $U$ is the vibrational energy of the molecule, and $V_{\text {slab }}$ is the potential energy of the slab.

The slab energy is defined by

$$
E_{\text {slab }}=T_{\text {slab }}+V_{\text {slab }}=\frac{1}{2} \sum_{\alpha=1}^{N_{\text {slab }}} m_{\alpha} \boldsymbol{v}_{\alpha}^{2}+V_{\text {slab }}
$$

where $T_{\text {slab }}$ is the kinetic energy of the slab, $V_{\text {slab }}$ is its vibrational energy, $\boldsymbol{v}_{i}$ and $m_{i}$ are the velocity and the mass of slab atom $\alpha$, respectively, and $N_{\text {slab }}$ is the number of slab atoms.

The energy of the molecule $E_{\mathrm{mol}}$ is given by (41), and its decomposition in the different components and how they can be calculated from the atomic positions and velocities are 
shown in the previous section (4.2.1). But for preparing the molecule in its initial state it was not necessary to consider the coupling between the rotational and vibrational dof. However, this coupling plays a role after the projectile is scattered from the slab and it begins to rotate. The coupling is due to the dependence of $R$ and $U$ on $r$. The dependence of the rotational energy on the bond distance can be analyzed in terms of the expansion of the angular velocity $\boldsymbol{\omega}$ and the inertia tensor $\underline{\underline{I}}$ in terms of the molecular equilibrium configuration characterized by $\underline{\boldsymbol{I}_{0}}$, which rotates with angular velocity $\boldsymbol{\omega}_{0}$, such as:

$$
\underline{\underline{I}}=\underline{\underline{I_{0}}}+\Delta \underline{\underline{I}}, \quad \omega=\omega_{0}+\Delta \omega
$$

yielding

$$
\begin{aligned}
& R=\frac{1}{2} \omega \cdot \underline{\underline{\boldsymbol{I}}} \cdot \omega=\frac{1}{2}\left(\omega_{0}+\Delta \omega\right) \cdot\left(\underline{\underline{I_{0}}}+\Delta \underline{\underline{I}}\right) \cdot\left(\omega_{0}+\Delta \omega\right)= \\
& \frac{1}{2}\left[\omega_{0} \cdot \underline{\underline{I_{0}}} \cdot \omega_{0}+2 \omega_{0} \cdot \underline{\underline{I_{0}}} \cdot \Delta \omega+2 \omega_{0} \cdot \Delta \underline{\underline{I}} \cdot \Delta \omega+\Delta \omega \cdot \underline{\underline{I_{0}}} \cdot \Delta \omega+2 \omega_{0} \cdot \Delta \underline{\underline{I}} \cdot \Delta \omega+\Delta \omega \cdot \Delta \underline{\underline{I}} \cdot \Delta \omega\right]
\end{aligned}
$$

So only the first term the second line of depends entirely on the equilibrium configuration and does not vary with $\boldsymbol{r}$ and thus is constant in time and is the pure rotational energy $R_{0}$. All other terms are also depending on the actual bond distance $\boldsymbol{r}$ and will therefore vary in time. They can be summarized in an energy contribution $E_{\mathrm{rv}}$ which is exchanged between the rotational and vibrational dof.

To assign the vibrational energy to the upper energy limit of a $v$ state is then calculated via

$$
E_{\text {Morse }}(v)+\frac{\left(E_{\text {Morse }}(v+1)+E_{\text {Morse }}(v)\right)}{2}
$$

and the lower limit as

$$
E_{\text {Morse }}(\nu-1)+\frac{\left(E_{\text {Morse }}(v)+E_{\text {Morse }}(\nu-1)\right)}{2},
$$

where the bottom limit for $v=0$ is 0 . In principle one could also use a model — rigid rotor - to assign the rotational energy to certain rotational state but as the number of trajectories for every set of initial conditions is very small the resolution of individual rotational states does not lead to a good comparison to experimental results. Thus the rotational energy is not assigned to the corresponding quantum states. 


\subsection{Analysis of the translational, rotational and vibrational energy transfer from trajectories}

The output from the simulations delivers the positions, the velocities and the forces acting on atoms in the simulation box. To analyze the energy transfer pathways during collisions between projectile and surface it is necessary to be able to assign the energy content to the degrees of freedom of interest. The most important dofs in this context are translational, rotational and vibrational dof of the incident diatomic molecule. Here I derive the formulas allowing this to be done and use an AIMD trajectory representing the scattering of a highly vibrationally excited $\mathrm{CO}(\nu=22)$ molecule from $\mathrm{Au}(111)$ surface.

Fig. 9 shows the time-dependence of the kinetic and potential energy contributions (see previous section) along a trajectory representing the scattering of a highly vibrationally excited $\mathrm{CO}(v=22)$ molecule from the $\mathrm{Au}(111)$ surface. The simulation was done with FHI-aims, and the incidence conditions were: incidence energy $E_{\text {in }}=0.58 \mathrm{eV}$, incidence angle $\theta_{\text {in }}=0^{\circ}$, initial rotational energy $R_{\text {in }}=0$, and initial vibrational energy $E_{\text {vib,in }}=$ $5.170 \mathrm{eV}$ corresponding to $v=22$ (see the maximum value of $T_{\mathrm{v}}$ in Fig. 9 before collision). Due to the collision with the surface, the energy initially deposited in the translational and vibrational dofs of the molecule is transferred into the rotational (blue solid curve in Fig. 9), slab (red solid line) and translational (black solid line) dofs. The total energy is conserved along the trajectory (dark gray curve) with an accuracy of about $100 \mathrm{meV}$.

To obtain a quantitative description of the energy transfer, the initial and final values of translational, rotational, and vibrational energies of the molecule as well as the energy of a slab are necessary. I denote the initial values by the subscript 'in' and the final values by "f". For example, the translational energy loss $T_{\text {mol,loss }}$ is

$$
T_{\mathrm{mol}, \mathrm{loss}}=T_{\mathrm{mol}, \mathrm{in}}-T_{\mathrm{mol}, \mathrm{f}}
$$

where $T_{\mathrm{mol}, \text { in }}$ and $T_{\mathrm{mol}, \mathrm{f}}$ are the initial and the final (after scattering) translational energies of the molecule, respectively.

There is no problem in determining the translational energy via the first equation of (42) and the first equation of (43), since the quantities $\boldsymbol{v}_{A}$ and $\boldsymbol{v}_{B}$ are provided by the simulation output. This delivers the value of $1.110 \mathrm{eV}$ for the final translational energy $T_{f}$, which gives

$$
\Delta T=T_{f}-E_{\text {in }}
$$

as shown in Fig. 9. 


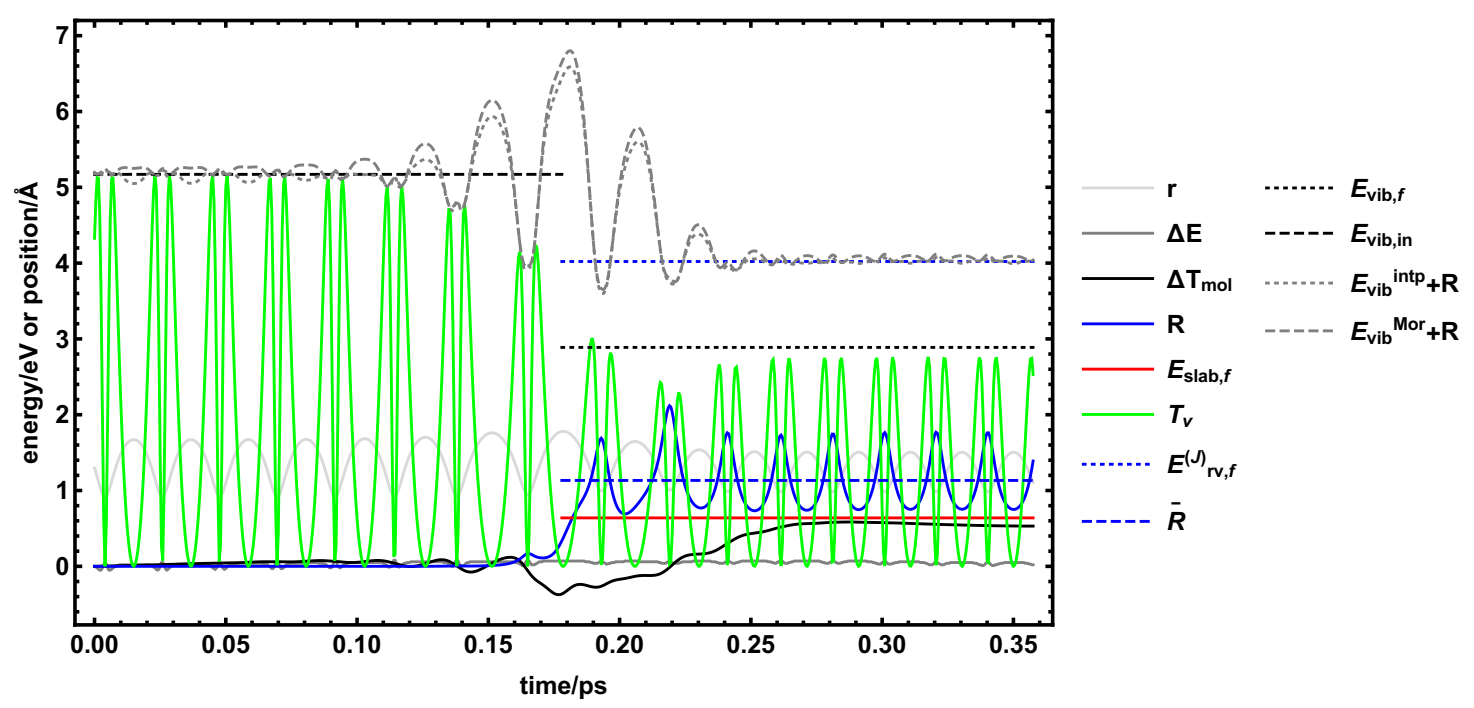

Fig. 9: Energy exchange along a trajectory simulating $\operatorname{CO}(\nu=22)$ scattering from $\mathrm{Au}(111)$ at incidence energy $E_{\mathrm{in}}=0.58 \mathrm{eV}$ and normal incidence. Shown are: change of the total energy $\Delta E_{\text {tot }}=E_{\text {tot }}-E_{\text {tot,in }}$ (dark gray solid line), change of translational energy $\Delta T=T_{\text {mol }}-E_{\text {in }}$ (black solid line), rotational energy $R$ (blue solid line), kinetic vibrational energy $T_{\mathrm{v}}$ (green solid line), initial vibrational energy $E_{\mathbf{v i b}}$, in (black dashed line), final vibrational-rotational energy $E_{\mathbf{r v , f}}^{(J)}=\bar{R}_{\mathbf{f}}+E_{\mathbf{v i b}, \mathbf{f}}$ (blue dotted line), which consists of the average final rotational energy $\bar{R}_{\mathrm{f}}$ and the final vibrational energy $E_{\text {vib,f }}\left(\right.$ black dashed line), final slab energy $E_{\text {slab,f }}($ red solid line); the inter nuclear distance $|r|$ (light gray solid line).

The rotational energy can be determined via the second equation of (42) and (44). As the rotational energy depends on the bond distance $r=|\boldsymbol{r}|$ it oscillates out of phase with the bond distance (see Fig. 9): $R$ (blue solid line) has a minimum when $r$ (light gray solid line) has a maximum and vice versa.

Fig. 10 shows the contributions into $R$ for the trajectory shown in Fig. 9. Before the collision with the surface all contributions are zero. Only the rotational energy of the equilibrium configuration $\boldsymbol{\omega}_{0} \cdot \boldsymbol{I}_{0} \cdot \boldsymbol{\omega}_{0}$ is constant. The strongest oscillating contributions are due to the terms, which depend on $\Delta \boldsymbol{I}$ (green solid, green dotted and green dashed lines), but they partially cancel each other out.

Thus, the total rotational energy of the molecule is oscillating (black solid line), and the final rotational energy $\bar{R}_{\mathrm{f}}$ is defined as the value averaged over the vibrational period (the distance between neighboring maxima of the black solid line in Fig. 10)

The vibrational energy $E_{\mathrm{vib}}$ of the molecule consists of the kinetic $T_{\mathrm{v}}$ and potential $U$ energy contributions (see (41)). $T_{\mathrm{V}}$ can be easily calculated using the third Eqs. of (42) and (43). Unfortunately, the DFT calculations provide only the total potential energy of the system, and one has to apply additional considerations to extract the values of the vibrational potential. 


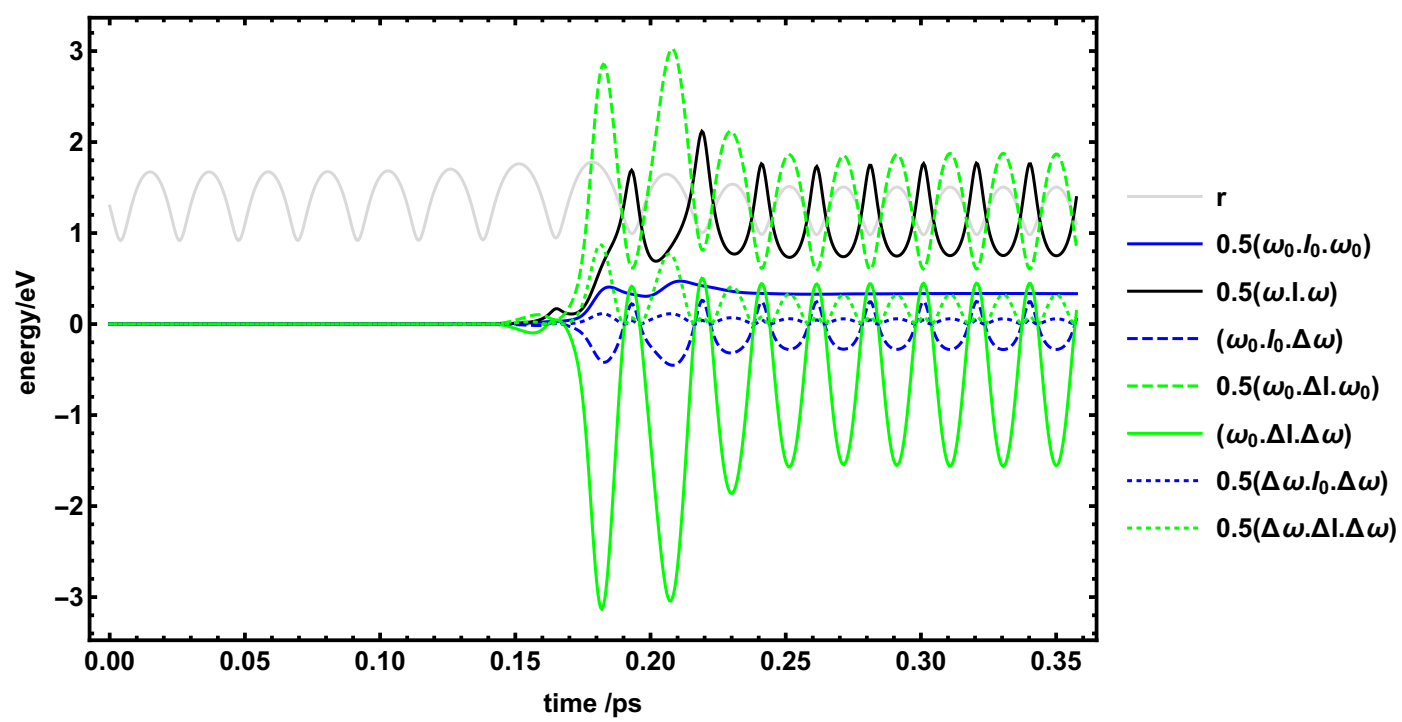

Fig. 10: Different contributions to the rotational energy, of the trajectory displayed in Fig. 9 are represented. Shown are: rotational energy of the equilibrium configuration $0.5 \omega_{0} \cdot I_{0} \cdot \omega_{0}$ (blue solid line), $\omega_{0} \cdot I_{0} \cdot \Delta \omega$ (blue dashed line), $0.5 \Delta \omega \cdot I_{0} \cdot \Delta \omega$ (blue dotted line), $\omega_{0} \cdot \Delta I \cdot \Delta \omega$ (green solid line), $\omega_{0} \cdot \Delta I \cdot \Delta \omega_{0}$ (green dashed line), $\Delta \omega \cdot \Delta I \cdot \Delta \omega$ (green dotted line), the sum of all these contributions (black solid line) and the bond distance $r$ as light gray line.

Consider a molecule far away from the surface, when the molecule-slab interaction $V_{\text {int }}=0$ and then its internal (ro-vibrational) energy

$$
E_{\mathrm{rv}}^{(J)}=R(r)+T_{\mathrm{v}}^{(J)}(r)+U(r)
$$

does not depend on the vibrational coordinate $r$. Here, the upper index $J$ indicates the dependence on the rotational state.

Fig. 11 shows the contributions into the ro-vibrational energy for two cases: a nonrotating molecule, $J=0$ (black curve), and $J>0$ (red curve). In the former case, the rotational energy is zero and the ro-vibrational energy is equal to the vibrational one. Applying this to (60), we get

$$
E_{\mathrm{rv}}^{(0)}=E_{\mathrm{vib}}=T_{\mathrm{v}}^{(0)}(r)+U(r) .
$$

As follows from the above definitions, the difference between two curves in Fig. 11 gives the rotational energy which is larger at the inner turning point due to its dependence on the vibrational coordinate $r$. This behavior can also be observed in Fig. 9 (compare blue and light gray solid lines).

In the absence of rotation, the vibrational motion occurs on the potential $U$ (black line in Fig. 11). The total vibrational energy of the molecule is constant and is defined by the 


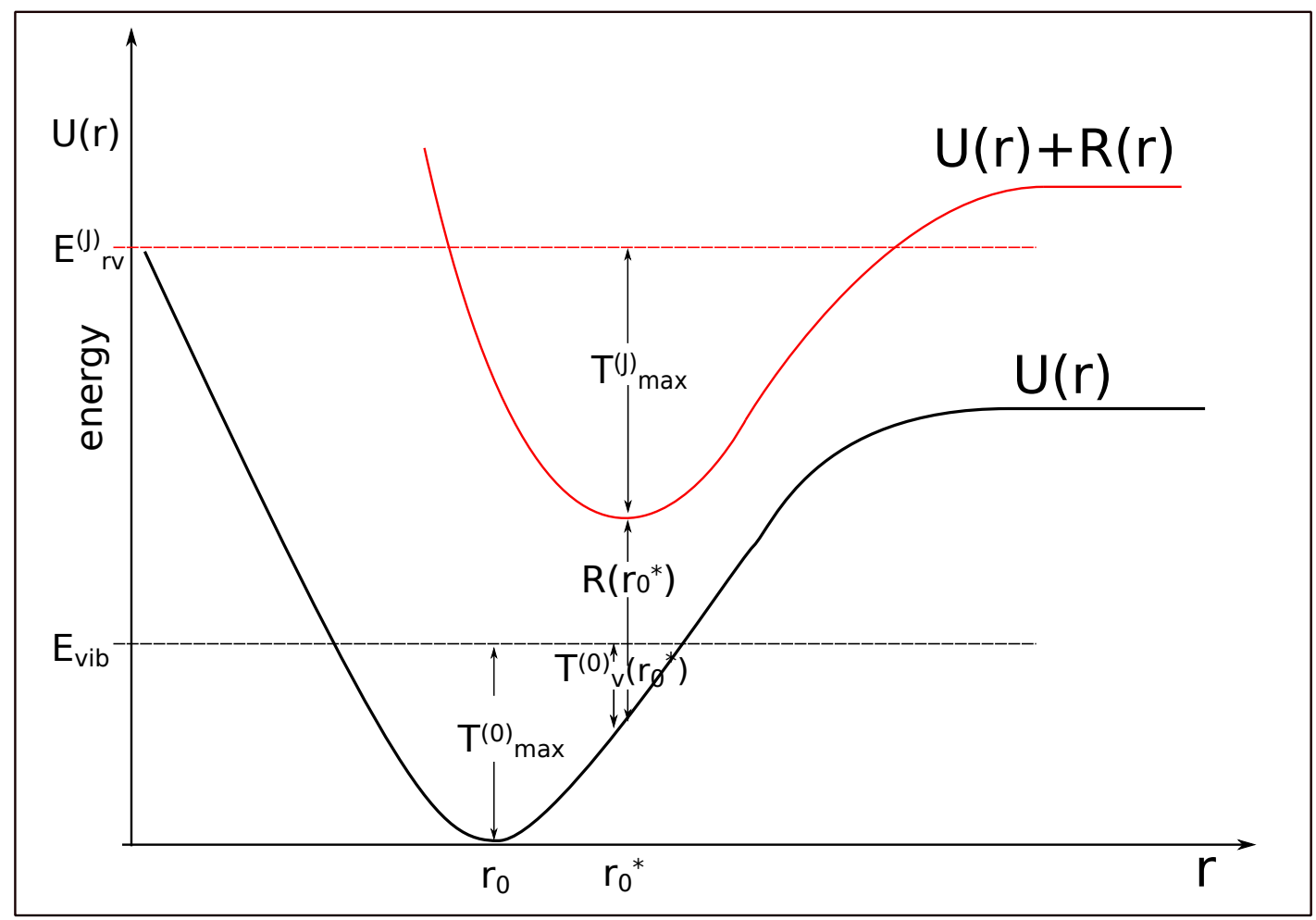

Fig. 11: Potential energy $U(r)$ of a diatomic molecule as function on the internuclear distance. The black curve shows the rotational ground state and the red curve shows a rotational excited state. The arrows and the vertical dotted lines indicate different energy contributions to the vibrational $E_{\mathrm{vib}}$ and the vibrational-rotational energy $E_{\mathrm{rv}}^{(J)}$ at different bond distances.

maximum value of the vibrational kinetic energy $T_{\mathrm{v}}^{(0)}\left(r_{0}\right)=T_{\max }^{(0)}$, which is reached at the potential minimum at $r_{0}$. Then Eq. (61) becomes the following:

$$
E_{\mathrm{vib}}=T_{\mathrm{v}}^{(0)}\left(r_{0}\right)=T_{\text {max }}^{(0)}
$$

which allows us to determine the potential energy curve by substituting it into Eq. ((61)):

$$
U(r)=E_{\mathrm{vib}}-T_{\mathrm{v}}^{(0)}(r)=K_{\max }^{(0)}-T_{\mathrm{v}}^{(0)}(r)
$$

For the rotating molecule the situation is more complicated due to the rotationalvibrational coupling, as it is discussed when considering Fig. 10. Now the system moves on the effective potential $U(r)+R(r)$ (red curve in Fig. 11) with new equilibrium geometry $r_{0}^{*}$. Note, that vibrational kinetic energy has a maximum at this position and its value can be extracted from the trajectory data (see green solid line in Fig. 9). Then, calculating the potential energy contribution for this new geometry from (63)

$$
U\left(r_{0}^{*}\right)=T_{\max }^{(0)}-T_{\mathrm{v}}^{(0)}\left(r_{0}^{*}\right),
$$


we arrive to the ro-vibrational energy of the molecule

$$
E_{\mathrm{rv}}^{(J)}=R\left(r_{0}^{*}\right)+T_{\mathrm{v}}^{(J)}\left(r_{0}^{*}\right)+U\left(r_{0}^{*}\right)
$$

which is independent on the vibrational coordinate $r$.

The procedure described above allows us to determine the final vibrational energy of the scattered molecule using the averaged rotational energy $\bar{R}$ as

$$
E_{\mathrm{vib}, \mathrm{f}}=E_{\mathrm{rv}}^{(J)}-\bar{R}
$$

As the total energy of the molecule is determined, we can finally calculate the slab energy substituting (65) in (51):

$$
E_{\text {slab }}=E_{\text {tot }}-T-E_{\mathrm{rv}}^{(J)} \text {. }
$$

So we have derived a procedure to obtain the initial and final energies for all dofs of the molecule and the slab. Hence, we are able to construct the final energy distributions and shed light on the energy transfer between relevant dofs.

Tab. 1: initial energies $E_{\mathrm{in}}$, final energies $E_{\mathrm{f}}$ and energy loss $E_{\text {loss }}$ of the different dofs of the molecule, $E_{\mathbf{m o l}}, E_{\mathbf{s l a b}}$ and $E_{\mathbf{t o t}}$ of the trajectory from Fig. 9 are represented.

\begin{tabular}{cccc}
\hline & $E_{\text {in }} / \mathrm{eV}$ & $E_{\mathrm{f}} / \mathrm{eV}$ & $E_{\text {loss }} / \mathrm{eV}$ \\
\hline$T$ & 0.580 & 1.110 & -0.530 \\
$R$ & 0 & 1.215 & -1.215 \\
$E_{\text {vib }}$ & 5.170 & 2.806 & 2.364 \\
$E_{\text {mol }}$ & 5.750 & 5.131 & 0.620 \\
$E_{\text {slab }}$ & $-1.9287163680 \times 10^{7}$ & $-1.9287163041 \times 10^{7}$ & -0.639 \\
$E_{\text {tot }}$ & $-1.9287157930 \times 10^{7}$ & $-1.9287157910 \times 10^{7}$ & -0.02 \\
\hline
\end{tabular}

Tab. 1 shows the initial, final (scattered) energy and energy loss for the different degrees of freedom of the system. The $4^{\text {th }}$ column shows that the vibrational energy lost in the collision is mostly distributed to the other dofs of the molecule and a fraction of $28 \%$ is transfered into the phonons of the slab.

Since the determination of the vibrational energy of the molecule is the most critical point in the analysis of the energies, I am going to explain why I have chosen the procedure described above. To determine $U$ one has to calculate the molecular energy as function of $r$. Then there are two possibilities to proceed: first one can fit the obtained DFT data with Morse function and second one can interpolate those data. Fig. 9 shows the sum of rotational and vibrational energy $R$ and $E_{\text {vib }}$ for both approaches as dotted gray $\left(E_{\mathrm{vib}}^{\text {Morse }}+R\right)$ and dashed gray lines $\left(E_{\mathrm{vib}}^{\mathrm{intp}}+R\right)$, respectively. The representation of 
$E_{\mathrm{vib}}$ by the Morse fit shows oscillations at the beginning and at the end of the trajectory at about $200 \mathrm{meV}$. The Interpolation of the DFT data performs better than the Morse fit, but it shows oscillations at the beginning of the trajectory too. So the above described procedure, taking the maximum of $T_{\mathrm{v}}$, seems to be a good approach to handle this issue.

\subsection{Transition dipole moment of carbon monoxide on surfaces}

Here I will describe a method to calculate the transition dipole moment on surfaces. But beforehand I give the general definition of this quantity and how it is calculated in molecules. This is followed by a description of problems which occur when the transition dipole moment is calculated in periodic systems, such as surfaces, and which solutions are available to tackle these problems.

The transition dipole moment is a very important quantity when describing interaction between electromagnetic radiation and matter [134]. It gives the possibility of a transition between two molecular states, e.g. electronic or vibrational states and is related to the life time of a molecule in its excited state. The latter is relatively easily accessible from spectroscopic experiments. In quantum mechanics the transition dipole moment between two states $m$ and $n$ is defined as

$$
\boldsymbol{\mu}_{n m}(\boldsymbol{R})=\int_{-\infty}^{\infty} \Psi_{m}^{*}(\boldsymbol{R}) \hat{\boldsymbol{\mu}}(\boldsymbol{R}) \Psi_{n}(\boldsymbol{R}) d \boldsymbol{R}
$$

where $\Psi_{m}$ and $\Psi_{n}$ represent the wave function of $n$ and $m$, respectively and $\hat{\boldsymbol{\mu}}$ the dipole moment operator. All of them depend on the coordinate $\boldsymbol{R}$ which describes the major changes in a coordinate during the transition. For example $\boldsymbol{R}$ can be a vibrational coordinate or a molecular bond distance. $\hat{\boldsymbol{\mu}}$ is the quantum mechanical analogue to the classical dipole moment

$$
\boldsymbol{\mu}=\sum_{i} \mathrm{q}_{i} \boldsymbol{R}_{i}
$$

Here, $\mathrm{q}_{i}$ and $R_{i}$ are the charge and the position vector of the $i^{\text {th }}$ particle.

Having defined all these quantities the path to get the transition dipole moment of a molecule on metal surfaces lies straight before us. With respect to (68) we need expressions for the wave functions for the molecule in the relevant states as well as an expression for the dipole moment operator for the system under consideration.

Although the solution of (69) in connection with the particle charge seems to be very simple in classics it is one of the most difficult problems in quantum mechanics. It concerns the question how we can assign an electron to a certain atom? Since, the 
position of an electron cannot be determined due to the HEISENBERG uncertainty principle [135].

Two approaches were developed to handle this problem. The first one is based on population analysis of orbitals or basis functions and was developed by MULLIKEN [136], whereas the second frequently used approach is based on the analysis of the electron density or more precisely its Laplacian. This method was introduced by Bader and is known as Theory of Atoms in Molecules [109]. As the latter approach is also used in another context within this thesis and is explained in section 3.4.

So the population analysis of Mulliken uses the dot product of the overlap $\underline{\underline{S}}$ with $S_{i j}=$ $\left\langle\phi_{i} \mid \phi_{j}\right\rangle$ and the density matrix $\underline{\underline{D}}$ with $D_{i j}=c_{i}^{*} c_{j}$ of all occupied molecular orbitals which are expanded in basis functions $\phi_{j}$ giving the numbers of electrons $\mathrm{N}_{\text {elec }}$. Because every basis function sits on a certain atom the trace of $\underline{\underline{D}} \cdot \underline{\underline{S}}$ gives the $\mathrm{N}_{\text {elec }}$ belonging to an atom, and the off-diagonal elements give the electrons shared by two atoms. The simplest scheme to divide these shared electrons is an equal distribution between the two atoms, which is used by Mulliken's approach. Having distributed all electrons to their atoms one can calculate the charge of each atom.

The next point in the computation of the dipole moment concerns the position of the particle, in particular the origin to which the position refers. For a neutral system or molecule the origin is arbitrary, as can be easily shown using (69), because summing up of all charges leads to zero. So there is no problem in calculating $\boldsymbol{\mu}$ for the neutral CO molecule, but when the molecule approaches the surface a charge transfer between them will occur. So the molecule is not neutral anymore and we have to tackle the mentioned problem. One solution is to calculate the moment for a fixed reference point, e.g. the COM of the molecule or of the total system. A second approach is just to calculate $\boldsymbol{\mu}$ for the whole system, i.e. molecule and surface, which has a net charge of zero again.

All said considerations of calculations of dipole moments were with regards to molecules or clusters and not periodic systems like surface which we wish to calculate. Charges in periodic systems are difficult to describe. Because the number of atoms in a bulk or surface is infinite the dipole moment cannot simply be computed via (69). To overcome this, one defines a dipole moment per unit volume or area. This quantity is the electric polarization with this we are able to calculate the total dipole moment for our moleculesurface system, and then by using an appropriate wave function we can compute the transition moment of molecule at the metal surface.

But using polarization does not solve the following problem which applies to periodic structures. This can be described by the concept of multi-valuedness of bulk polarization [137]. It describes the problem that the value of the polarization depends of 
the choice of the origin and the number of unit cells which are considered. This last problem can be avoided by calculating the change in the polarization, instead of the polarization itself, between two configurations where the atoms are slightly shifted to one another. This change is independent of the origin of the unit cell and serves as a reference configuration to compute a molecule on a surface which is easily defined, e.g. the molecule in its adsorption minimum.

To compute the transition dipole moment we have to know the wave function of the system and the transition dipole moment operator, see (68). I consider an approximation where wave functions describing the surface are supposed to be unchanged and just the molecular contribution to the total wave function will be affected. So it seems to be justified to only consider the latter. Of course this approximation needs to be checked by comparing the obtained with corresponding experimental data. For diatomic molecules which can be described as Morse oscillator one can gain analytic expression for wave functions as a function of the vibrational state [138, 139] as

$$
\psi_{\text {Morse }, v}(q)=N_{\text {Morse }, v} q^{\lambda-\nu-\frac{1}{2}} \exp \left(q-\frac{1}{2}\right) L_{v}^{2 \lambda-2 v-1}(q)
$$

where $v$ denotes the eigenstates given by $v=0,1, \ldots, \lambda-\frac{1}{2}$ and $q=2 \lambda \exp \left(-\alpha_{\mathrm{M}}\left(r-r_{\mathrm{eq}}\right)\right)$, where the parameter $\lambda=\frac{\sqrt{\mu \mathrm{D}_{\mathrm{e}}}}{\alpha_{\mathrm{M}} \hbar}$, where $\mu$ is the reduced mass of the diatomic molecule. The normalization factor can be calculated as

$$
N_{\text {Morse }, v}=\sqrt{\frac{v !(2 \lambda-2 v-1)}{\Gamma(2 \lambda-n)}} .
$$

$L_{v}^{2 \lambda-2 v-1}(q)$ is a generalized Laguerre polynomial. Thus, the eigenfunction is completely described by analytically known functions and the Morse parameters $\alpha_{\mathrm{M}}, \mathrm{D}_{\mathrm{e}}$ and $r_{\mathrm{eq}}$ for a chosen eigenstate $v$.

The dipole moment operator in Eq. (68) is a vector and thus the polarization and its change, too. In all following considerations the change in polarization is projected onto the $\mathrm{CO}$ bond vector. To obtain an expression for the dipole moment operator the change in polarization is computed for different $\mathrm{CO}$ bond lengths. These data are then fitted to a cubic function as

$$
\widehat{\mu}(r):=\Delta \widehat{\mu}(x)=a x^{3}+b \Delta x^{2}+c x+d,
$$

where $\widehat{\mu}(x)$ is change in polarization (dipole moment per unit area) of the total system with respect to the minimum energy configuration, $x=r-r_{\mathrm{eq}}$, and $a, b, c$, and $d$ are the fit parameters, respectively. With the dipole moment operator and the wave functions one can compute the transition dipole moment. 
For example, to calculate the transition dipole moment of $\mathrm{CO}$ at a surface I use the following procedure: First, find the relaxed structure of $\mathrm{CO}$ adsorbed at the surface. Second, calculate the energy and charges on atoms for different molecular bond distances. Third, fit the data obtained to analytical functions (46) and (72) defining Morse-parameters and hence the wave functions - and transition dipole moment operator. And finally, calculate the transition dipole matrix elements for different transitions from $n$ to $m$ using (68). 


\section{Phonon spectra for $\mathrm{Au}(111), \operatorname{Ag}(111)$ and Ag-covered Au(111) surfaces}

\subsection{Introduction}

The investigation of phonons, vibrations of atoms in a solid, is of interest when looking into energy transfer of molecules to surfaces, because phonons provide an energy reservoir with which the molecule can exchange energy during the encounter with the surface. This coupling between molecular motion and vibration of the atoms in the solid is purely mechanical and no non-adiabatic effects need to be considered. In the case of the scattering of $\mathrm{CO}(\nu=2)$ and $\mathrm{NO}(v=2)$ from $\mathrm{Au}(111)$ and Ag-covered $\mathrm{Au}(111)$ the experimentally observed final translational energy distributions show a stronger loss in energy for Ag-covered surfaces than for a pure Au(111) surface as can be seen in Fig. 12. Here the mean final translational energy of the scattered molecules is shown as function of the thickness of the Ag-layers on the Au(111) surface [39]. The mean final energy does not change when the layer thickness is larger than $4 \mathrm{ML}$. This observation seems to be due to differences in the properties of the different surfaces as both molecules show this behavior. To find an explanation for this observation a closer look on the properties of the different surfaces is necessary but also the interactions between molecule and the surfaces can provide an explanation. This is a motion to study the properties of the phonon spectra with ab initio methods. 

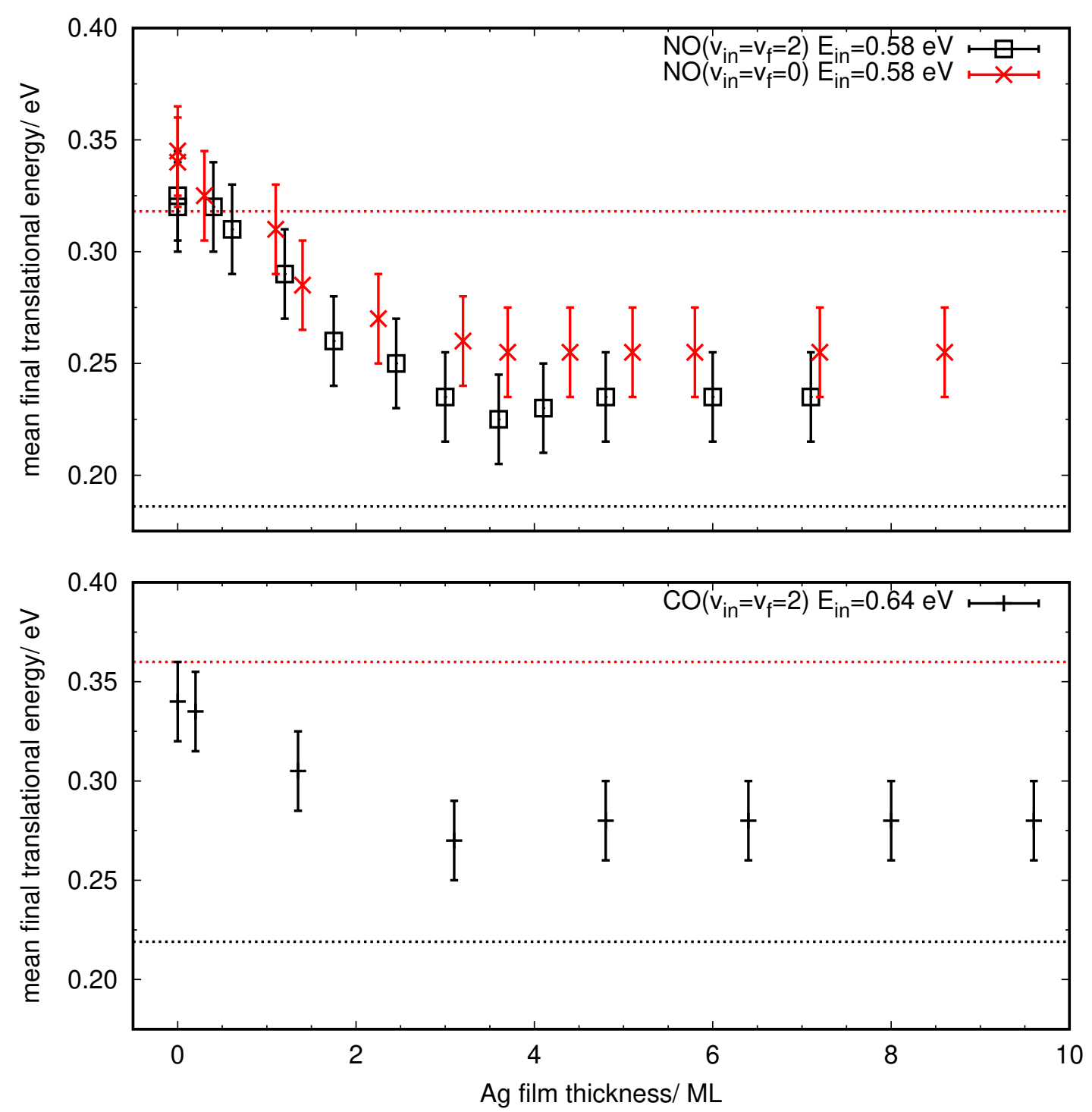

Fig. 12: Experimentally determined mean final translational energy vs. the Ag-film thickness for scattered $\operatorname{NO}(v=2 \rightarrow 2)$ and $\operatorname{CO}(v=2 \rightarrow 2)$ in the vibrationally elastic and $\operatorname{NO}(v=2 \rightarrow 0)$ in vibrationally inelastic case. The red and black dotted lines indicate the Baule limit for $\mathrm{Au}(111)$ and Ag(11), respectively. 


\subsection{Results}

To obtain the phonon spectra for the different surfaces the Phonopy-code [106] was used. As described in section 3.3, the phonons are calculated via the finite-difference approach. Phonopy was used to create the displaced input configurations as well as to calculate all vibrations from the forces of the displaced configurations. The force calculations were actually done with VASP. The DOS of the spectra are given as the number of vibrations per unit cell and per frequency. I calculated phonon spectra for the (111)-surfaces as well as for bulk gold and silver.

For the DFT calculations I used the GGA functionals RPBE and as comparison the revised Perdew Burke Ernzerhofer for solids, a GGA functional (PBEsol) [140] because it is especially designed to reproduce properties of solids. The basis set to the wave function includes plane waves up to an $E_{\text {cut }}$ of $400 \mathrm{eV}$ and the electron-core interaction was represented by PAWs. The fractional electronic occupation was described by an MP1 function with a width of $0.2 \mathrm{eV}$. Since sampling of the reciprocal space influences strongly the calculation of the phonon spectra the results were tested against variation of the $\boldsymbol{k}$-point grid size. Hence the grid was varied from $4 \times 4 \times 4$ to $32 \times 32 \times 32$. In contrast to the determination of the equilibrium lattice constant $a$ where the simulation cell consists of a single atom, this is not enough in the case of phonon spectra because displacement between different atoms is needed for the phonons. So I used a simulation cell containing 8 atoms, 27 atoms and a simple 1-D model with two atoms to represent the $f c c$-bulk. The atoms in simulation cell were translated along the $f c c$ lattice vectors, as given in Eq. (36). The 1-D chain model is not sufficient to describe the gold crystal, because the experimental Debye frequency of the crystal is not well reproduced. This is seen in Fig. 13a, where the Debye frequency is clearly higher than the highest frequencies of the calculated spectra. Further calculations with different $\boldsymbol{k}$-point grids show that the grid size has no strong influence on the DOS. A denser grid only shifts the first peak in the spectrum to slightly smaller frequencies and to higher numbers of states.

The DOS of the 3-D model crystals is different to the chain model as seen in Fig. 13b. Here the highest peak for the number of states is not around $1 \mathrm{THz}$ but around $3.5 \mathrm{THz}$. Furthermore, the DOS of bulk model is broader than the one of the chain, thus states with a higher frequency as $3.2 \mathrm{THz}$ are occupied. Increasing the number of atoms in the model from 8 to 27 leads to a slight shift of the whole DOS spectrum to lower frequencies. The size of $\boldsymbol{k}$-grid used for the force calculation does not change the shape of the spectrum significantly. The DOS spectra for the $\mathrm{p}(2 \times 2 \times 2)$ and the $\mathrm{p}(3 \times 3 \times 3)$ bulk show that the states with the highest energy have a frequency which is slightly higher 


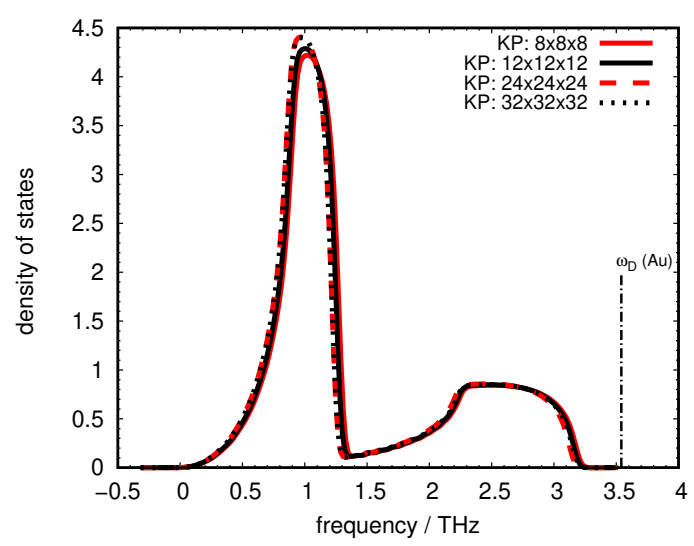

(a) Density of states of a simple 1-D chain consists of two atoms for different dense $k$-point grids. The vertical black dashed line indicates the experimental Debye frequency of a gold.

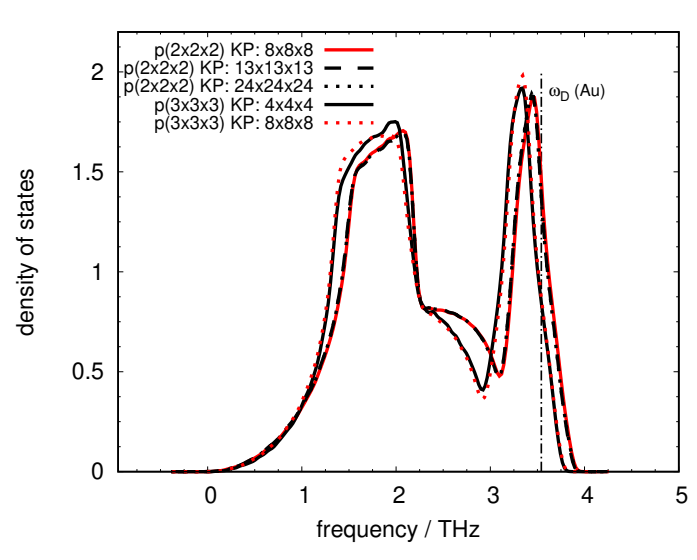

(b) Density of states of Au-bulk models consist of 8 and 27 atoms respectively, sampled with a different number of $k$-points. The vertical black dashed line again represents the Debye frequency of gold.

Fig. 13: Phonon spectra for a chain model (a) and two simulation cells (b) representing the $f c c$ bulk for different $k$-point grid sizes, calculated with RPBE.

than the Debye frequency of gold, which is $3.51 \mathrm{THz}$ [141], whereby the frequency of the larger bulk cell comes closer to that value.

Using the same bulk models for silver delivers a DOS, see Fig. 14, which has a comparable shape to that of gold, but its fastest phonons have a frequency smaller than the Debye frequency for that metal, which is $4.47 \mathrm{THz}$ [141]. But determining the DOS using the PBEsol functional - which is constructed to describe the properties of solids - shifts the complete spectrum to a higher frequency with maximal frequency of $5.5 \mathrm{THz}$, and therefore the fastest phonons have a frequency much higher than the Debye frequency. Thus, the maximum of the phonon spectrum calculated with RPBE is closer to the Debye frequency than that value obtained with PBEsol. Perhaps this reflects that the Debye model, on which the frequency is based, has some weaknesses to describe the phonon spectrum. Since PBEsol should in principle give good results for solid properties like phonon spectra.

Further the differences in the shape of the DOS between the $\mathrm{p}(2 \times 2 \times 2)$ (Fig. 14a) and the $\mathrm{p}(3 \times 3 \times 3)$ cells (Fig. $14 \mathrm{~b})$ are small compared to the shifts due to the change of the functional.

To calculate the phonon DOS of the different surfaces I started with a $\mathrm{p}(1 \times 1)$ cell with 4 layers and a vacuum distance of $20 \AA$ to build up the simulation cell. I used the input parameters as described above for the force calculations of this system. The $\boldsymbol{k}$-point sampling was tested for the $\mathrm{Ag}(111)$ case, and the outcome of those tests are presented in Fig. 15a.

The total appearance of the phonon DOS does not strongly change with increasing $\boldsymbol{k}$ point grid for the actual force calculation; the spectrum looks smoother and occurring 


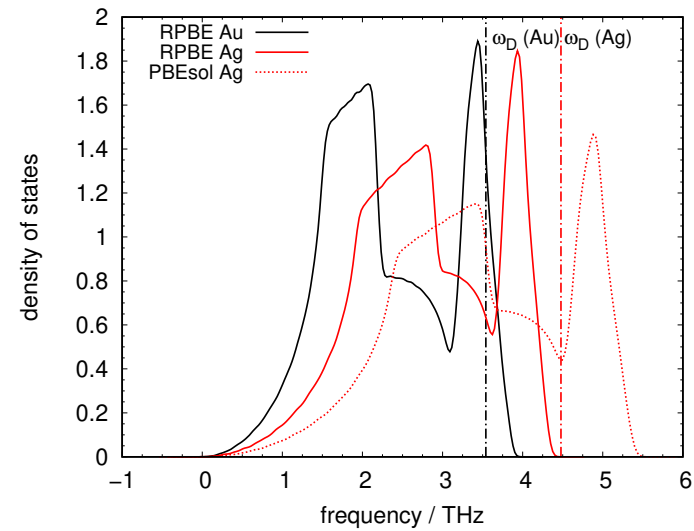

(a) Phonon DOS or a $\mathrm{p}(2 \times 2 \times 2)$ cell including 8 atoms using $k$-point grid of $24 \times 24 \times 24$.

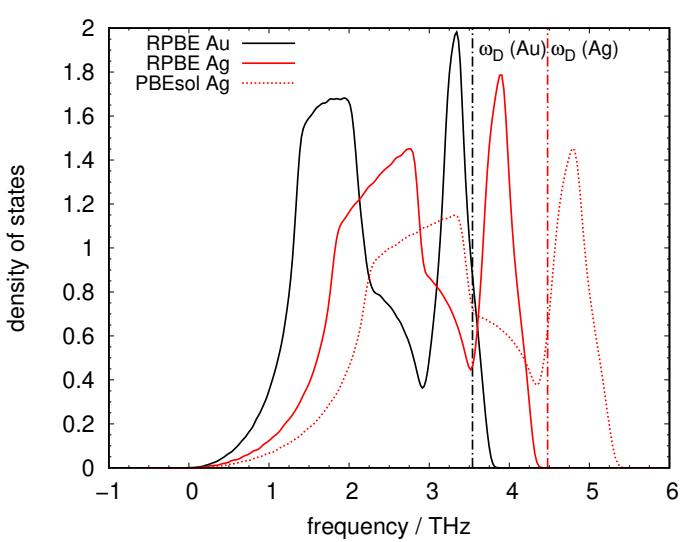

(b) Phonon DOS or a p $(3 \times 3 \times 3)$ cell including 27 atoms using a $k$-point grid of $8 \times 8 \times 8$.

Fig. 14: Phonon spectra for a bulk model including 8 (a) and 27 atoms (b) for gold and silver for RPBE represented by a black solid and a red solid line and for PBEsol by a red dotted line, respectively. The vertical black and the red dashed-dotted lines indicate the Debye frequencies of gold and silver, respectively.

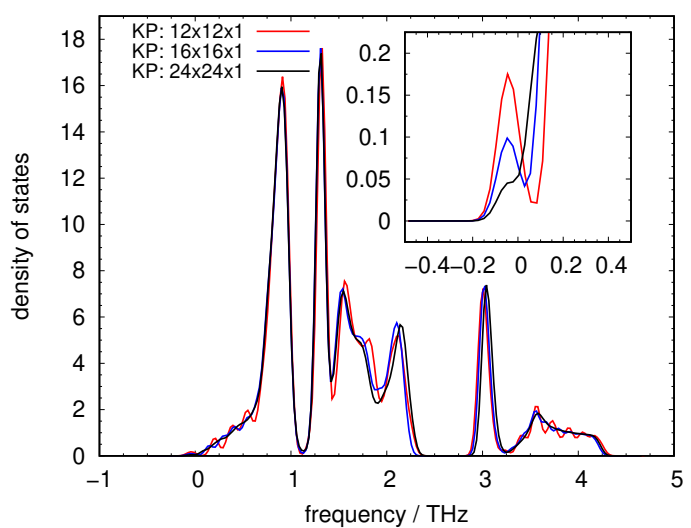

(a) Phonon DOS of a silver surface modeled by a p $(1 \times 1 \times 4)$ slab for different $k$-point grids, represented by red $(12 \times$ $12 \times 1)$, blue $(16 \times 16 \times 1)$ and black lines $(24 \times 24 \times 1)$. The in shows the DOS in a frequency range of -0.5 to $0.5 \mathrm{THz}$, to emphasize the differences of the spectra.

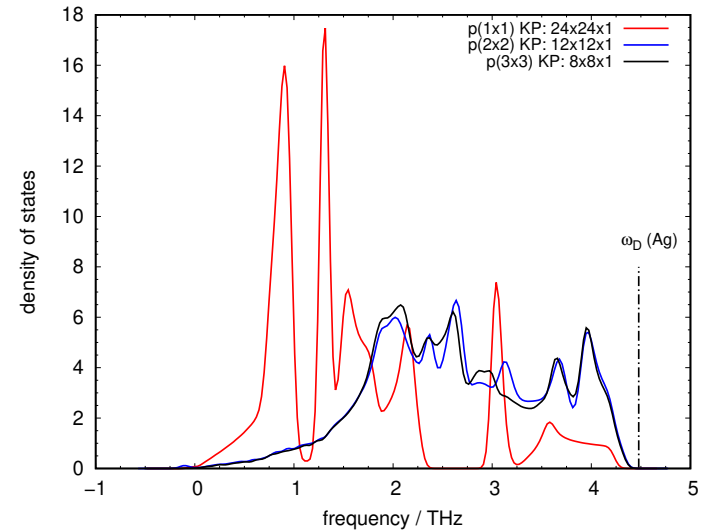

(b) Phonon DOS of silver surface modeled by a $\mathrm{p}(1 \times 1)$ (red line), a $\mathrm{p}(2 \times 2)$ (blue line) and a $\mathrm{p}(3 \times 3)$ slab (black line) with 4 layers, respectively. The vertical black dashed line indicates the Debye frequency of silver.

Fig. 15: Phonon DOS of a silver surface modeled by $\mathrm{p}(1 \times 1)$ slab with 4 layers for different $k$-point grids (a) and (b) modeled by a p $(1 \times 1), \mathbf{p}(2 \times 2)$, and $\mathbf{p}(3 \times 3)$ slab with 4 layers, respectively. 
oscillations are averaged out. But the inset of Fig. 15a shows that a denser grid reduces a small peak which occurs in the negative frequency range. Since negative frequencies are non-physical these are attributed to a not well-enough sampled reciprocal space leading to an incorrect force calculation. So although the $\boldsymbol{k}$-point sampling does not have a strong influence on the total resulting spectrum a $24 \times 24 \times 1$-grid was chosen for further force calculations to avoid the unreasonable artifacts.

Making the cell bigger in lateral, $x$ - and $y$-direction changes the overall appearance more strongly than the change of the $\boldsymbol{k}$-point grid. In the case of the $\mathrm{p}(1 \times 1)$ cell, states with frequencies about $1 \mathrm{THz}$ are much stronger populated than in that of the $\mathrm{p}(2 \times 2)$ where the population of the vibrational states does not show high peaks at certain frequencies and is much more distributed over a frequency range from $1.5 \mathrm{THz}$ to $4.2 \mathrm{THz}$, as shown in Fig. 15b. Around this latter frequency the population of states plummets to zero for the cells mentioned before as well as for the bigger $\mathrm{p}(3 \times 3)$ cell depicted as the black line in the same figure. The spectrum for the largest cell is very similar to that of the $\mathrm{p}(2 \times 2)$ cell. In case of the $\mathrm{p}(1 \times 1)$ cell no vibrations occur within a frequency range between 2 and $3 \mathrm{THz}$, so that the vibrations of the larger cells occurring in this range are along the $x$ - and $y$-direction. Despite the apparent differences between the $\mathrm{p}(1 \times 1)$ and the two other cells, the maximum frequencies which inherit solid vibrations are similar to each other and only slightly smaller than those of the $\mathrm{p}(2 \times 2)$ and $\mathrm{p}(3 \times 3)$ cell. So this feature of the spectrum does not depend on the size of the cell. After I checked the influence of the lateral size of the surface model on the phonon DOS I tested the influence of the variation of the number of layers. To do this I used a $\mathrm{p}(1 \times 1)$ cell and a $\boldsymbol{k}$-point grid of $24 \times 24 \times 1$ for the VASP calculations. I did calculations for five to seven layers and one with 11 layers. The latter calculations were done because here the atoms of the last layer are under that of the first layer and to see if a further increase of layers changes the appearance of the spectrum strongly. The resulting phonon spectra and the spectrum of a bulk model consisting of 27 atoms are shown in Fig. 16. Comparing the bulk model and the slabs clearly shows that the slab models inherit a higher number of states and the shape of the spectrum changes strongly, thus some frequencies between 2 and $3 \mathrm{THz}$ are occupied in the bulk but not or only slightly occupied in slab models. The obvious difference is between the spectra of the slabs with four, five and six layers because in the two latter cases phonons occur which inherit much higher frequency than those of the first case. So the five-layer slab shows vibrations with frequencies up to $8 \mathrm{THz}$ and the six-layer slab shows frequencies up to $6 \mathrm{THz}$. These changes in the appearance of the DOS are due to the fact that atoms at different positions are sitting in the two layers nearest to the vacuum (top and bottom layer), and therefore different and much faster vibrations are possible when the slab has an $A B C A B$ or $A B C A B C$ structure. The 
regular distance between the maximum occupied frequencies of the spectra may be due to the regular structure of the atoms, so the atom of the $5^{\text {th }}$ layer is $\frac{1}{3}$ closer to the atom of the top layer than that one of the $6^{\text {th }}$ layer. The models which have an $A B C A$ structure, as those with 4, 7 and 11 layers, have no phonons with a frequency higher than $4.3 \mathrm{THz}$, and the spectra go to zero at very similar frequencies. The width of the phonon spectra changes only slightly when the structure of the surface layers is the same. However, as the number of atoms increases when the number of layers goes up the DOS shows a higher number too.

Having tested the influence of the $\boldsymbol{k}$-point grid as well as the size of the surface cell in the lateral direction and number of surface layers on phonon DOS spectra of the surface, I chose a $\boldsymbol{k}$-point grid of $24 \times 24 \times 1$ and $\mathrm{p}(1 \times 1)$ cell with 4 layers to model the pure gold and Ag-covered surfaces. For this the latter were created by successively exchanging $\mathrm{Au}$ atoms with silver ones, starting with the top layer. The so obtained phonon DOS spectra of the different surfaces are shown in Fig. 17 . The spectra are shifted to higher frequencies when the Au atoms are replaced by Ag atoms. This is not just the case for each complete individual spectrum but in particular for the highest frequencies at which vibrations occur. These frequencies are very similar for the pure gold case (marked as 0 layer(s) Ag in Fig. 17) and the slab with 1 silver layer. For the slab with $2 \mathrm{Ag}$ layers this frequency is shifted more to the right, whereas the slab, consisting of $3 \mathrm{Ag}$ and $1 \mathrm{Au}$ layer, is shifted to a even higher frequency which is very close to that one of the pure silver slab. The Debye frequency $\omega_{\mathrm{D}}$ of gold is a bit lower than the maximum frequency of the pure gold surface whereas in case of silver $\omega_{\mathrm{D}}$ occurs at higher frequencies than the maximum frequencies of the phonons in the silver surface model.

In Fig. 18 the highest frequency of the phonon spectra, marked by a black arrow in Fig. 17, is plotted against the number of silver layers which the slab contains. The frequency shows a shift from the pure gold slab (0 Ag layers) to the slab containing 3 Ag layers, and here the frequency reaches a maximum and only changes slightly when the number of silver layers is further increased. This is similar to the trend in the experimentally determined mean final translational energy for scattered NO and CO which shows a change when the Ag-coating is increased from 0 to 4 ML, as seen in Fig. 12. 


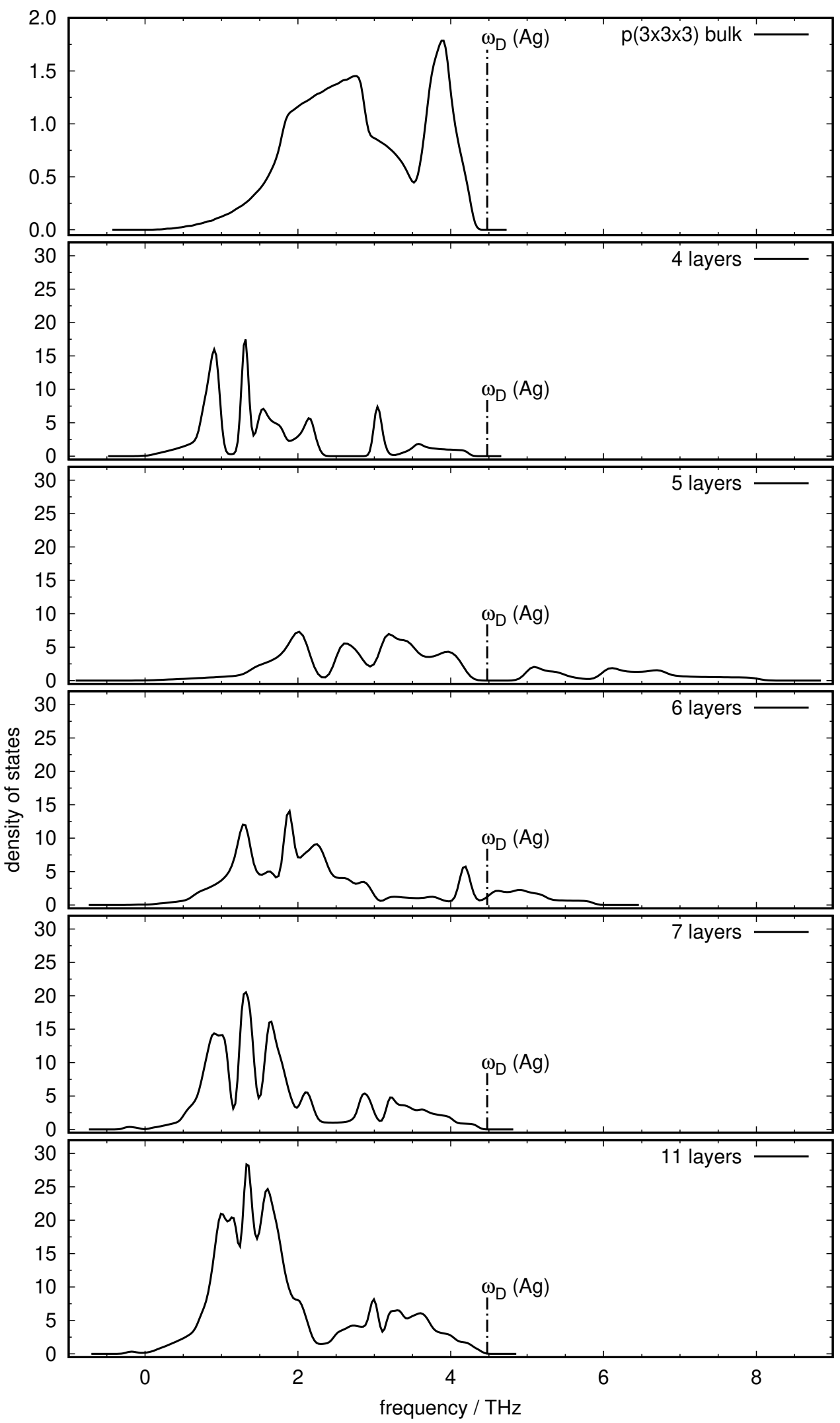

Fig. 16: Phonon DOS for an Ag su modeled as $p(3 \times 3 \times 3)$ cell (top) and $p(1 \times 1)$ slabs of Ag with 4 to 11 layers ( $2^{\text {nd }}$ to bottom panel). The vertical dashed-dotted line indicates the Debye frequency of $\mathrm{Ag}$. 


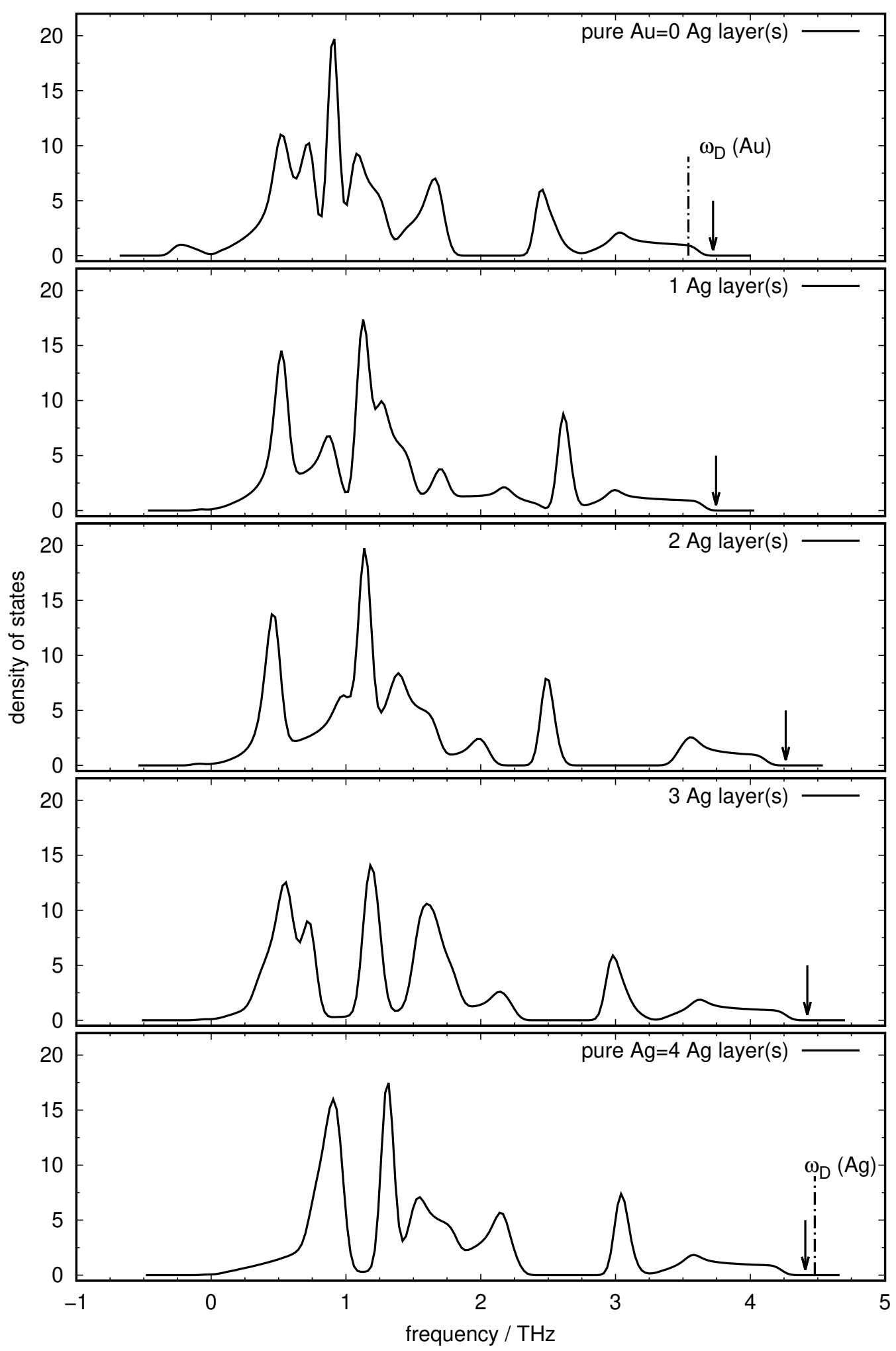

Fig. 17: Phonon DOS for a $p(1 \times 1)$ slab with 4 layers of different numbers of silver layers from 0 (upper panel) to 4 (bottom panel). The intermediate cases are represented in $2^{\text {nd }}, 3^{\text {rd }}$ and $4^{\text {th }}$ panels from top, respectively. The black dotted-dashed lines in the top and bottom panels are representing the Debye frequencies $\left(\omega_{D}\right)$ of gold and silver, and the arrows indicate the highest frequency of the corresponding phonon DOS. 


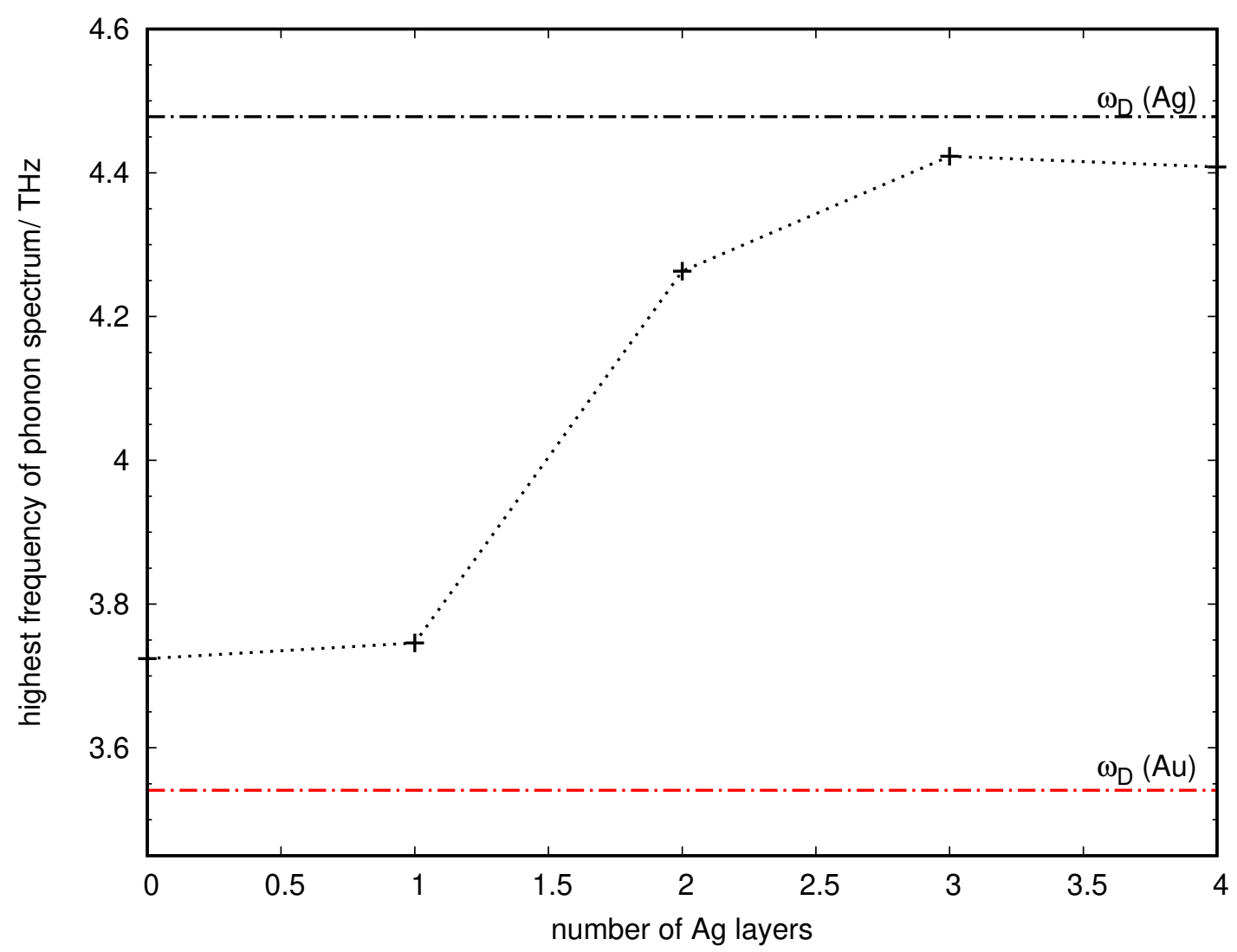

Fig. 18: Highest frequency of the phonon spectrum vs. the number of silver layers representing the slab. The dotted line is a guide line to the eyes. The red and black dashed-dotted lines representing the Debye frequencies of $\mathrm{Au}$ and Ag, respectively. 


\subsection{Conclusions}

The mechanical properties of the Au surfaces with the various numbers of Ag layers on top are systematically changing with the number of silver layers due to the different coupling between the phonons and the translational dof of an impinging molecule. So the translational inelasticity dependence of scattered CO and NO molecules on the Ag film thickness is due to the change of the phonon spectra from $\mathrm{Au}$ to Ag.

It seems to be unlikely that the differences in the final translational energy distribution of different coated surfaces are due to differences in the adsorption energy as the adsorption well depths of the Ag(111) and Ag-coated (111)-slabs are almost the same (see section 7.3). Furthermore, the translational energy distribution of AIMD simulations for scattering $\mathrm{CO}$ from these surfaces are quite similar to those obtained in the experiment (see section 8.3). Therefore, a purely mechanical picture seems to be a reasonable explanation for the differences in the translational energy transfer between the molecule and the different surfaces.

Thus the differences in the phonon motion of the surfaces lead to different couplings to the translational dof of the molecule. The results reported in this chapter were mostly published in [39]. 


\section{Minimum energy pathways for dissociation and oxidation of diatomics on metal surfaces}

\subsection{Introduction}

In order to investigate the influence of vibrational energy in a dissociation reaction of a diatomic molecule it is crucial to know the configuration of the TS. Since, if one knows the configuration of the TS, one can make predictions on how vibrational energy promotes the dissociation according to Polanyi's rules [37]. The rules state that a reaction will be promoted via vibrational excitation when the transition state looks more like the reaction products (late barrier). In contrast, if the transition state looks more like the reactants (early barrier), a higher translational energy will promote the reaction. Another aspect in doing MEP calculations is to get the energetics of these dissociation processes, so that one can calculate the reaction rate from transition state theory.

\subsection{Hydrogen halides on $\mathrm{Au}(111)$ and $\mathrm{Ag}(111)$}

I investigated the MEP for dissociation of $\mathrm{HF}$ and $\mathrm{HCl}$ on $\mathrm{Au}(111)$ and $\mathrm{Ag}(111)$. I also tested the influence of surface atom motion in the case of $\mathrm{HCl}$ on $\mathrm{Au}(111)$. Before I did the calculation to find the TS, I had to find the initial and the final states, i.e. the minimum energy structures for the molecule and the separated atoms adsorbed on the surface. These structures were obtained via geometry optimization carried out with FHI-aims. These calculations were done in the spin-unpolarized approach of the GGA using the RPBE-functional. Taking this approach seems to be reasonable because although the dissociation leads to unpaired electrons it happens on a metal slab, where all electrons are delocalized over the slab. This approach also needs a smaller amount of computational time. The reciprocal space was sampled by a $4 \times 4 \times 1 \boldsymbol{k}$-point grid and the occupation of the bands was modeled by a Gaussian function and width $\sigma$ of $0.2 \mathrm{eV}$. 


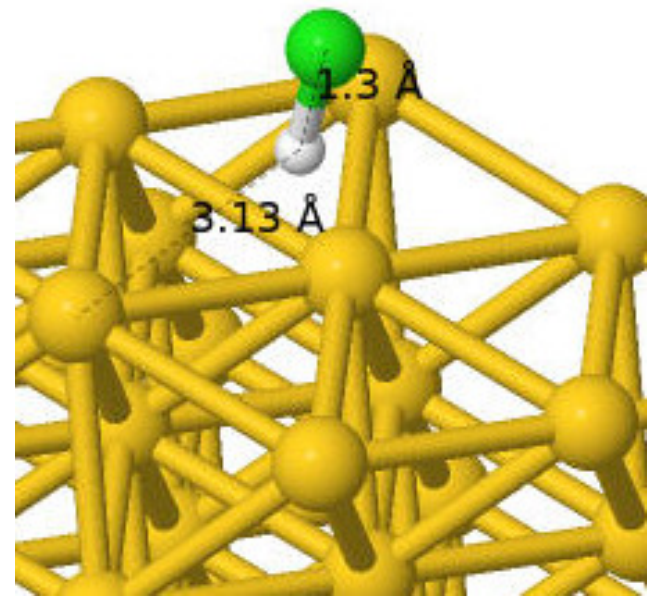

(a) Initial state for $\mathrm{HCl}$ on the $f c c$ site of the rigid $\mathrm{Au}(111)$ slab.

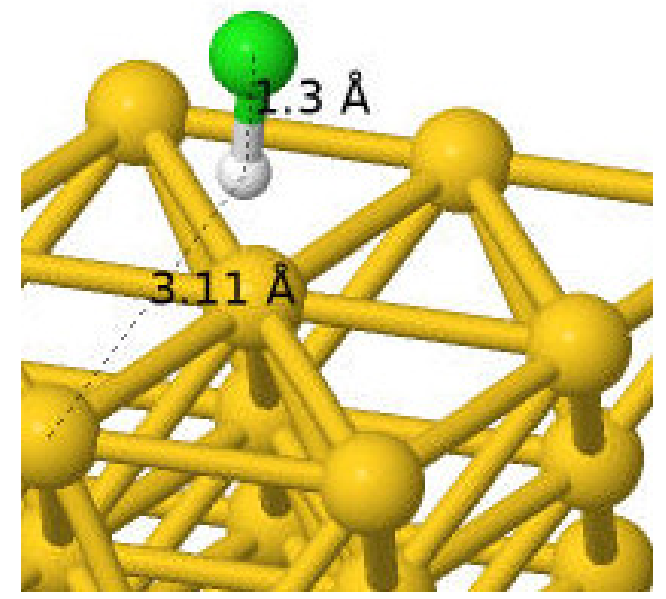

(c) Initial state for $\mathrm{HCl}$ on the $f c c$ site of the movable Au(111) slab.

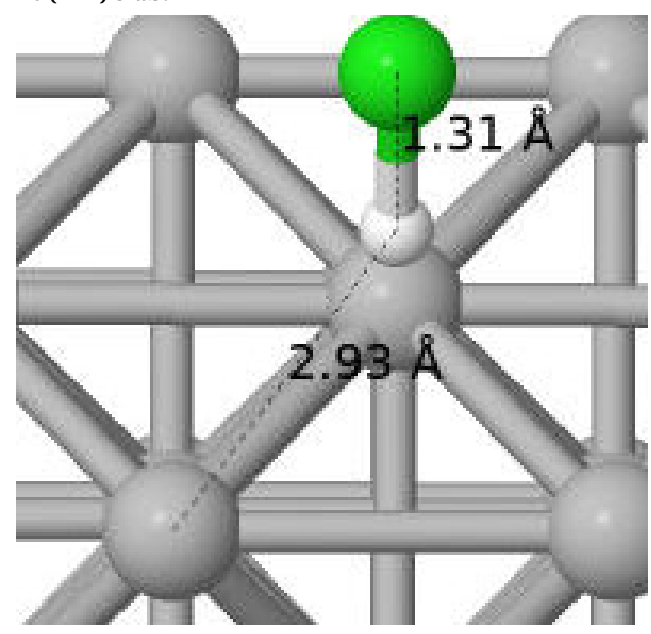

(e) Initial state for $\mathrm{HCl}$ on the hcp of the rigid $\mathrm{Ag}(111)$ slab.

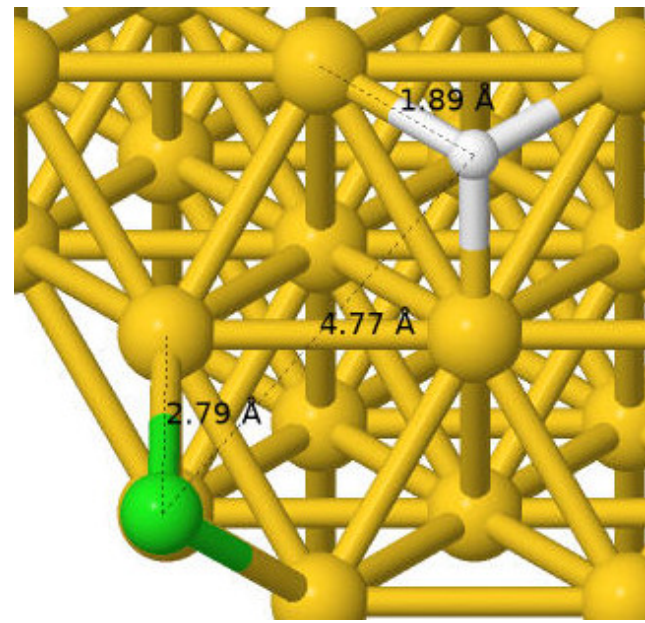

(b) Final state for $\mathrm{HCl}$ on the rigid $\mathrm{Au}(111)$ slab. $\mathrm{H}$ sits on $f c c$ and $\mathrm{Cl}$ sits on hcp site.

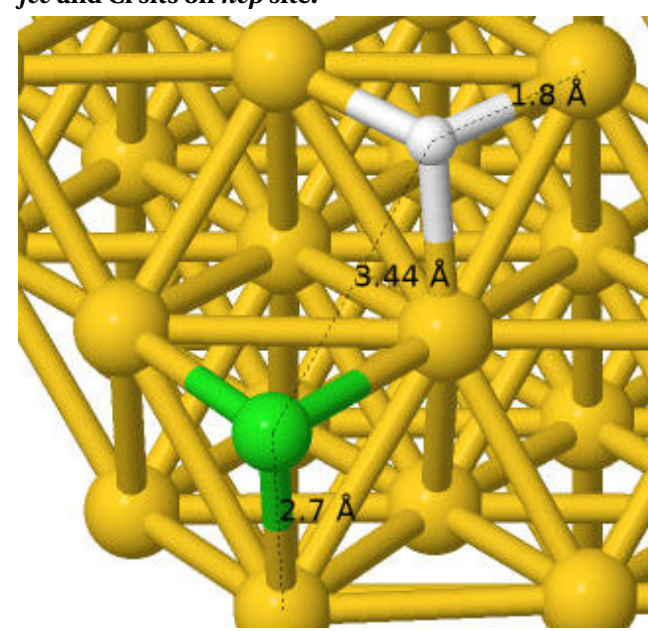

(d) Final state for $\mathrm{HCl}$ on the movable Au(111) slab. $\mathrm{H}$ and $\mathrm{Cl}$ sit both on $f c c$ site.

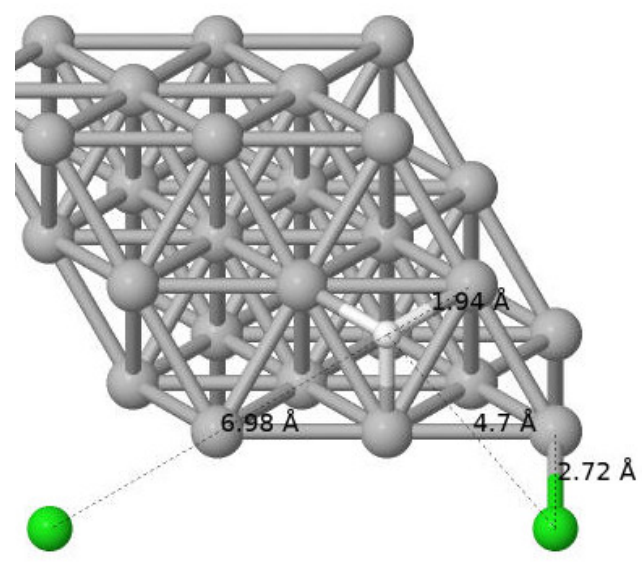

(f) Final state for $\mathrm{HCl}$ on the $\mathrm{Ag}(111)$ slab. $\mathrm{H}$ and $\mathrm{Cl}$ sit on the $f c c$ site.

Fig. 19: Initial (a, c, e) and final states (b, d, f) for $\mathrm{HCl} / \mathrm{Au}(111)$ (rigid slab, top), HCl/Au(111) (movable slab, center) and $\mathrm{HCl} / \mathrm{Ag}(111)$ (rigid slab, bottom). The hydrogen, chlorine, gold and silver atoms are represented by white, dark green, dark yellow and gray spheres, respectively. The numbers indicate the distances between certain atoms. 
The predefined light-setting was used for the basis sets of all species. The simulation cell consisted of a $\mathrm{p}(3 \times 3)$ slab with 4 layers of the corresponding metals and a length of the vacuum layer of $20 \AA$. The cells were built up by an optimized lattice constant of $4.20 \AA$ for $\mathrm{Au}$ and Ag. During the geometry optimization the atoms of three uppermost layers were allowed to move and the optimization was stopped when the forces acting on all atoms were $<0.01 \mathrm{eV}^{-1}$.

For the separated atoms I tried different initial configurations, so I set both atoms on different adsorption sites, to make sure the structure is obtained which corresponds to the minimum energy. The energy minimum structures were confirmed via calculations with tight-settings for the basis sets. The MEP was sampled with ten images and was calculated by the string-method as implemented in the aimsChain utility. The stringmethod should be preferred to the NEB-approach [105]. For the total path a convergence criterion of $<0.2 \mathrm{eVA}^{-1}$ and to determine the transition state more accurately a criterion for the climbing image of $<0.05 \mathrm{eV}^{-1}$ was used. For these calculations the same input parameters as for the geometry optimizations were used.

In the minimum energy configuration $\mathrm{HF}$ and $\mathrm{HCl}$ on $\mathrm{Au}(111)$ and $\mathrm{Ag}(111)$ are oriented with $\mathrm{H}$ towards the surface. In case of $\mathrm{HCl}$ on $\mathrm{Au}(111)$ and $\mathrm{Ag}(111)$ the molecule is bound either on the $h c p$ or on the $f c c$ site (see Fig. 19), whereas HF sits on both surfaces on the hcp site, which can be seen in Fig. 20.

In all cases the molecule is perpendicular to the surface and more than $2 \AA$ away from the surface. HF comes closer to the surface than $\mathrm{HCl}$, and in case of $\mathrm{HF} / \mathrm{Ag}(111)$ it has the distance of $2.3 \AA$. In the cases of the separated atoms both atoms sit either on the $f c c$ or the $h c p$ site where the distance between atoms is always more than $4.7 \AA$. Hence the molecule is clearly dissociated, and in the case of $\mathrm{HCl} / \mathrm{Au}(111)$ with movable slab atoms the distance is $3.44 \AA$. Furthermore, in Tab. 2 several structural quantities for the initial, final and TS configurations are presented.

The TS configurations for the different systems are shown in Fig. 21. The configurations for $\mathrm{HCl}$ on $\mathrm{Au}(111)$ in the case of the movable (Fig. 21a) and the rigid slab atoms (Fig. 21b) look quite similar. Thus, the chlorine atom bridges to surface atoms and the hydrogen sits slightly shifted on the top site. The distance between $\mathrm{H}$ and $\mathrm{Cl} d_{\mathrm{HCl}}$ is $2.07 \AA$ (rigid slab atoms) and $2.0 \AA$ (movable slab atoms), respectively. For the TS configuration of the HF dissociation on $\mathrm{Au}(111)$, see Fig. 21c, the hydrogen atom sits on the bridge and the fluorine sits shifted on the top site. Here the distance between the two atoms is $d_{\mathrm{HF}}=1.84 \AA$ and thus strongly elongated compared to the bond distance in the initial state, which is $0.94 \AA$. The TS configurations of the reaction of $\mathrm{HCl}$ (Fig. 21d) and $\mathrm{HF}$ (Fig. 21e) on $\mathrm{Ag}(111)$ are different. Thus, in the former case the hydrogen bridges two slab atoms and chlorine sits a bit shifted on the top site, whereas in the case of HF 
Tab. 2: Structural parameters for the initial (upper section), TS (middle section), and final (bottom section) configurations and the corresponding energies for the hydrogen halides on $\mathrm{Au}(111)$ and $\operatorname{Ag}(111) . \theta_{\mathrm{HX}}$ is the angle between the bond distance and the surface normal. $d_{\mathrm{H}-\mathrm{M}}$ or $d_{\mathrm{X}-\mathrm{M}}$ are the distances between hydrogen and the halide atom to the next surface atom. $z_{\mathrm{H}}$ and $z_{\mathrm{X}}$ are the $z$ coordinates of hydrogen and the halide atoms, respectively. The energy is referenced to the one of the initial state, which value is given below.

\begin{tabular}{|c|c|c|c|c|c|c|c|}
\hline System & $d_{\mathrm{HX}} / \AA$ & $\theta_{\mathrm{HX}} /^{\circ}$ & $z_{\mathrm{H}} / \AA$ & $z_{X} / \AA$ & $d_{\mathrm{H}-\mathrm{M}} / \AA$ & $d_{\mathrm{X}-\mathrm{M}} / \AA$ & energy/ eV \\
\hline $\mathrm{HCl} / \mathrm{Au}(111)^{\dagger}$ & 1.3 & 0 & 2.62 & 2.92 & 3.11 & 4.26 & $0^{\mathrm{a}}$ \\
\hline $\mathrm{HCl} / \mathrm{Au}(111)$ & 1.3 & 0 & 2.62 & 2.92 & 3.13 & 4.27 & $0^{\mathrm{b}}$ \\
\hline $\mathrm{HCl} / \mathrm{Ag}(111)$ & 1.31 & 0 & 2.47 & 3.77 & 2.93 & 4.10 & $0^{\mathrm{c}}$ \\
\hline $\mathrm{HF} / \mathrm{Au}(111)$ & 0.94 & 0 & 2.38 & 3.23 & 2.93 & 3.73 & $0^{\mathrm{d}}$ \\
\hline $\mathrm{HF} / \mathrm{Ag}(111)$ & 0.94 & 3 & 2.34 & 3.28 & 2.71 & 3.55 & $0^{\mathrm{e}}$ \\
\hline $\mathrm{HCl} / \mathrm{Au}(111)^{\dagger}$ & 2.0 & 60 & 1.42 & 2.36 & 1.65 & 2.73 & 0.90 \\
\hline $\mathrm{HCl} / \mathrm{Au}(111)$ & 2.07 & 115 & 1.54 & 2.42 & 1.63 & 2.73 & 0.93 \\
\hline $\mathrm{HCl} / \mathrm{Ag}(111)$ & 1.88 & 134 & 1.17 & 2.47 & 1.93 & 2.72 & 0.81 \\
\hline $\mathrm{HF} / \mathrm{Au}(111)$ & 1.84 & 123 & 1.06 & 2.06 & 1.83 & 2.22 & 1.83 \\
\hline $\mathrm{HF} / \mathrm{Ag}(111)$ & 1.59 & 121 & 1.88 & 2.34 & 1.94 & 2.55 & 1.53 \\
\hline $\mathrm{HCl} / \mathrm{Au}(111)^{\dagger}$ & 3.44 & 104 & 0.91 & 2.09 & 1.80 & 2.70 & 0.50 \\
\hline $\mathrm{HCl} / \mathrm{Au}(111)$ & 4.77 & 107 & 0.84 & 2.19 & 1.89 & 2.79 & 0.56 \\
\hline $\mathrm{HCl} / \mathrm{Ag}(111)$ & 4.70 & 105 & 0.86 & 2.06 & 1.94 & 2.72 & -0.14 \\
\hline $\mathrm{HF} / \mathrm{Au}(111)$ & 4.63 & 102 & 0.84 & 1.79 & 1.91 & 2.48 & 1.59 \\
\hline $\mathrm{HF} / \mathrm{Ag}(111)$ & 5.21 & 98 & 0.86 & 1.60 & 1.95 & 2.39 & 0.73 \\
\hline \multicolumn{8}{|c|}{${ }^{\dagger}:$ movable slab atoms } \\
\hline \multicolumn{2}{|c|}{$\begin{array}{l}\text { reference energy/ eV: } \\
c:-5.2828627643 \times 10^{6}\end{array}$} & \multicolumn{3}{|c|}{$\begin{array}{l}\text { a }:-1.92966775470 \times 10^{7} \\
\text { d }:-1.928681590 \times 10^{7}\end{array}$} & \multicolumn{3}{|c|}{$\begin{array}{l}\text { b }:-1.92966775487 \times 10^{7} \\
\text { e }:-5.2730011203 \times 10^{6}\end{array}$} \\
\hline
\end{tabular}




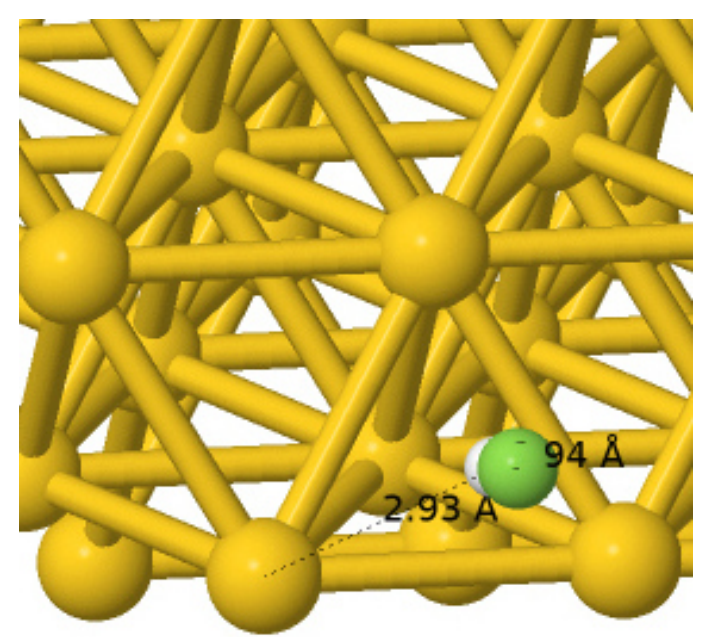

(a) Initial state for HF on the hcp site of Au(111).

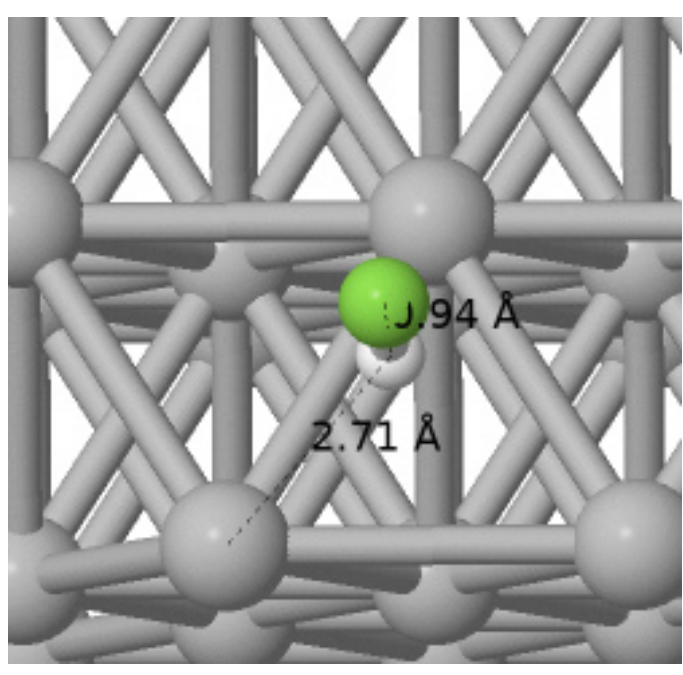

(c) Initial state for HF on the hcp site of $\mathrm{Ag}(111)$.

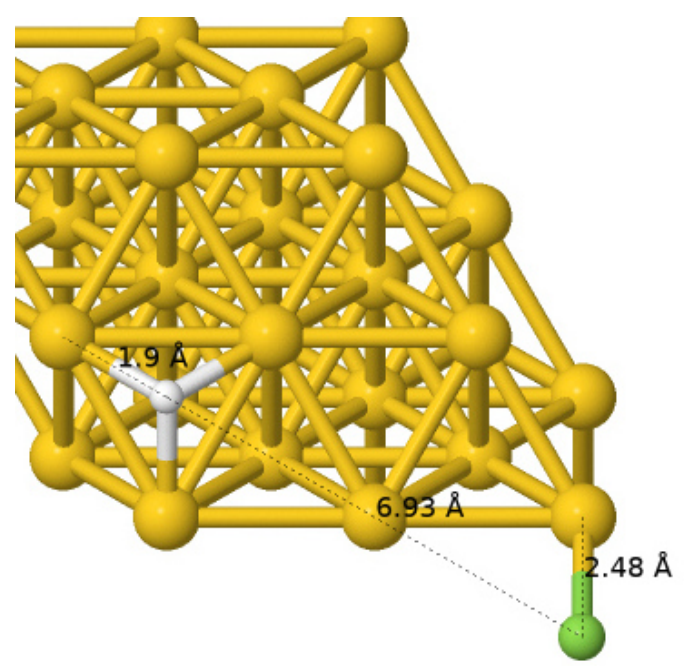

(b) Final state for HF on Au(111). H sits on the $f c c$ and F sits on the hcp site.

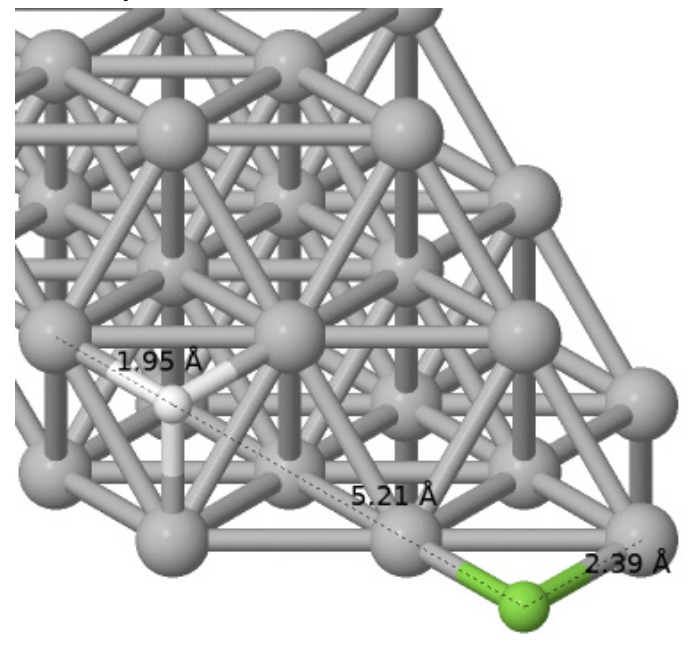

(d) Final state for HF on $\mathrm{Ag}(111) . \mathrm{H}$ and F sit both on $f c c$ sites.

Fig. 20: Initial ((a),(c)) and final states ((b),(d)) for HF on Au(111) (top) and HF on Ag(111) (bottom panel). The hydrogen, fluorine, gold and silver atoms are represented by white, light green, dark yellow and gray spheres, respectively. The numbers indicate distances between certain atoms. 
both atoms sit on a hallow site. Compared to the dissociation on the $\mathrm{Au}(111)$ surfaces the intermolecular distances in the case of $\mathrm{Ag}(111)$ are shorter, namely $d_{\mathrm{HCl}}=1.88 \AA$ and $d_{\mathrm{HF}}=1.59 \AA$. In each TS configuration the distance between both atoms is strongly elongated with respect to the distance in the initial configuration, and the configurations look more like the reaction products. According to Polanyi's rules this would suggest a late transition state and so the promotion of reaction via vibrational excitation. The smaller $\mathrm{F}$ atom comes closer to the surface than the larger $\mathrm{Cl}$ atom.

In Fig. 22 the MEP for the dissociation of $\mathrm{HCl}$ on $\mathrm{Au}(111)$ is shown for both a rigid slab and for one where the atoms of the upper three layers can move. For the rigid slab the barrier height is $927 \mathrm{meV}$ and so it is slightly higher than the barrier for the slab with the movable atoms ( $904 \mathrm{meV})$. The separated atoms on the surfaces also show also only small differences in the energies, that means $562 \mathrm{meV}$ in the case of the rigid slab and $497 \mathrm{meV}$ in the other case. Thus, the final states are higher in energy than the initial states, which is set as reference, hence $\mathrm{HCl}$ on the slab is more stable than the separated atoms. Since the structure of the moving surface did not change much in the MEP calculations and the resulting barriers and geometries are very similar to the frozen surface, only a rigid slab has been used for the calculations involving either HF or $\operatorname{Ag}(111)$.

In Fig. 23 the MEPs of the dissociation of $\mathrm{HCl}$ and $\mathrm{HF}$ on $\mathrm{Au}(111)$ (left) and $\mathrm{Ag}(111)$ (right) are shown. In the case of HF the reaction barrier is two times higher than that for $\mathrm{HCl}$ on both surfaces. So the barrier height is $1828 \mathrm{meV}$ for $\mathrm{HF}$ and only $907 \mathrm{meV}$ on $\mathrm{Au}(111)$, whereas the heights on the $\mathrm{Ag}(111)$ slab are $1530 \mathrm{meV}$ and $804 \mathrm{meV}$ for HF and $\mathrm{HCl}$, respectively. The separated atoms are more stable on $\mathrm{Ag}(111)$ than on $\mathrm{Au}(111)$. On the latter surface hydrogen and fluorine are around $1600 \mathrm{meV}$ higher in energy than the molecule on the surface, whereas the atoms on $\mathrm{Ag}(111)$ are only $500 \mathrm{meV}$ higher in energy than the molecule. The separated $\mathrm{H}$ and $\mathrm{Cl}$ atoms on $\mathrm{Au}(111)$ have an energy of $560 \mathrm{meV}$ with respect to the initial state, and so they are less stable than the two atoms on the silver surface, where the final configuration is about $140 \mathrm{meV}$ lower than the initial one. Hence this dissociation seems to be exothermic, whereas all other dissociation reactions are endothermic and the products are less stable than the adsorbed molecule. The barrier height of $0.91 \mathrm{eV}$ in the case of $\mathrm{HCl}$ on $\mathrm{Au}(111)$ was also found by KOLB et $a l$. using the NEB-method, as implemented in VASP, and using the RPBE-functional as well to characterize the dissociation reaction [142]. LIU and co-workers obtained a dissociation barrier of $0.61 \mathrm{eV}$ [36] using PW91 and more recently a value of $1.1 \mathrm{eV}$ employing RPBE [143]. As the latter functional is known to deliver higher reaction barriers the discrepancy seems to be due to the XC-functional. Therefore, we can say 


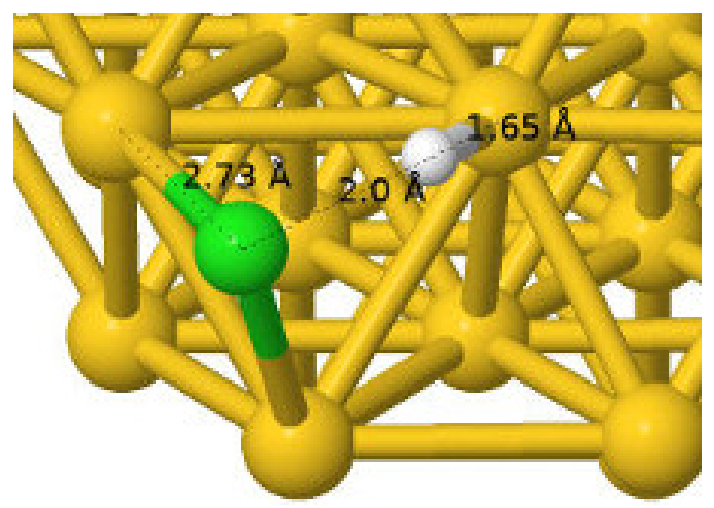

(a) HCl on Au(111) slab with movable atoms.

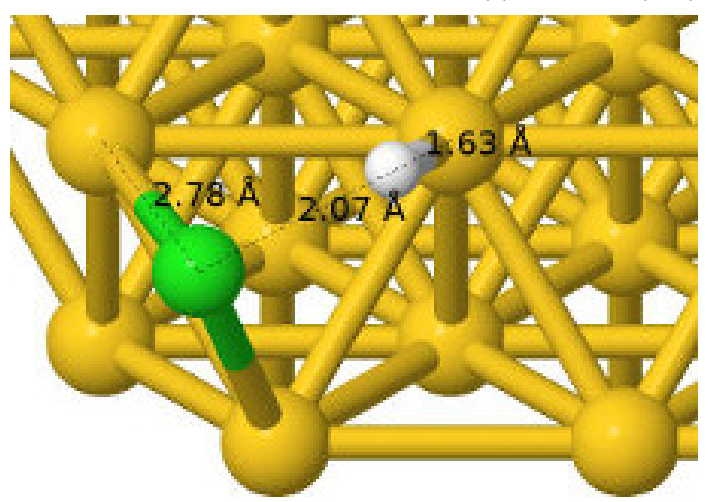

(b) HCl on the rigid Au(111) slab.

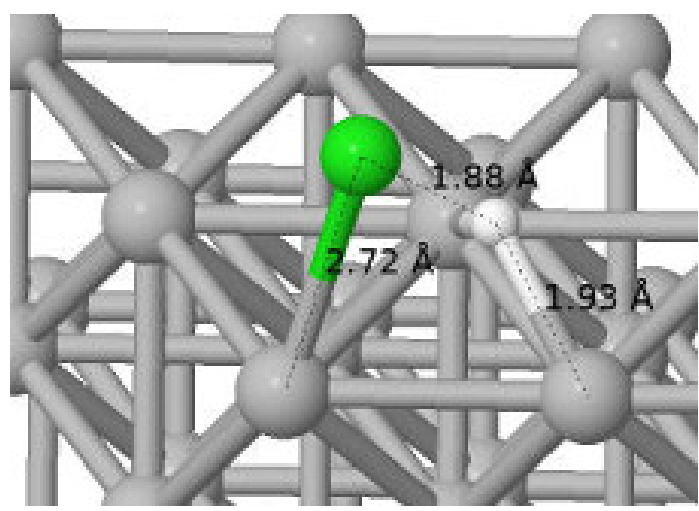

(d) $\mathrm{HCl}$ on the rigid $\mathrm{Ag}(111)$ slab.

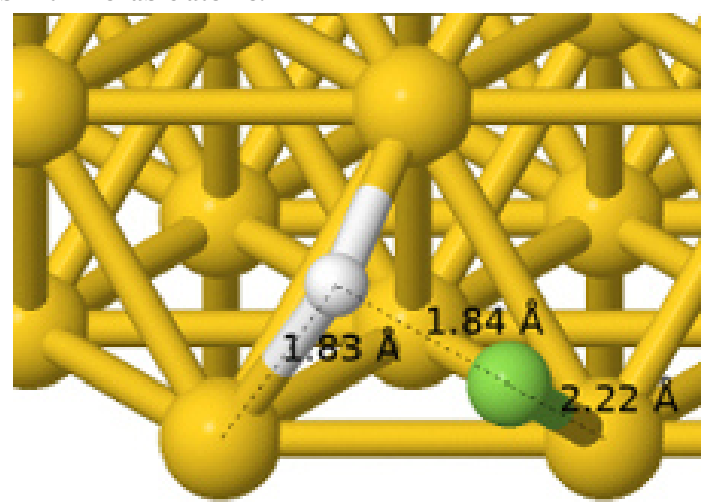

(c) HF on the rigid Au(111) slab.

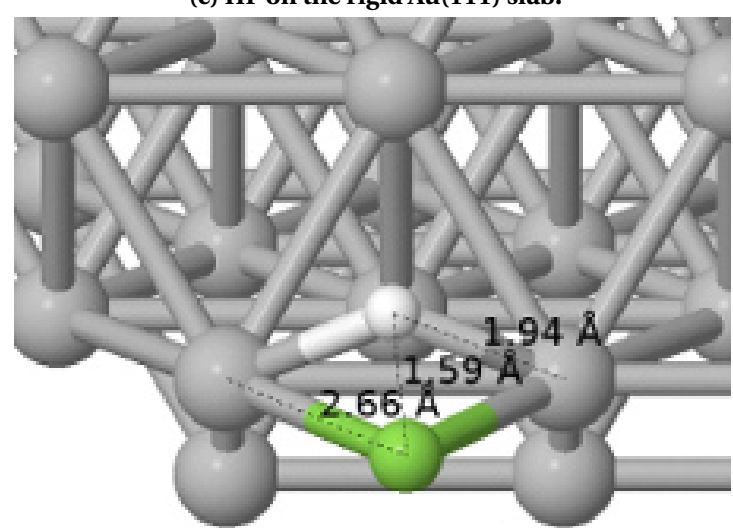

(e) HF on the rigid $\mathrm{Ag}(111)$ slab.

Fig. 21: TS configurations for the dissociation of: $\mathrm{HCl}$ on $\mathrm{Au}(111)$ with movable slab atoms (a), HCl on rigid Au(111) slab (b), HF on $\mathrm{Au(111)}$ (c), HCl on $\operatorname{Ag(111)}$ (d) and HF on $\operatorname{Ag(111).~The~hydrogen,~}$ chlorine, fluorine, gold and silver atoms are represented by white, dark and light green, dark yellow and gray spheres, respectively. The numbers indicate distances between different atoms. 


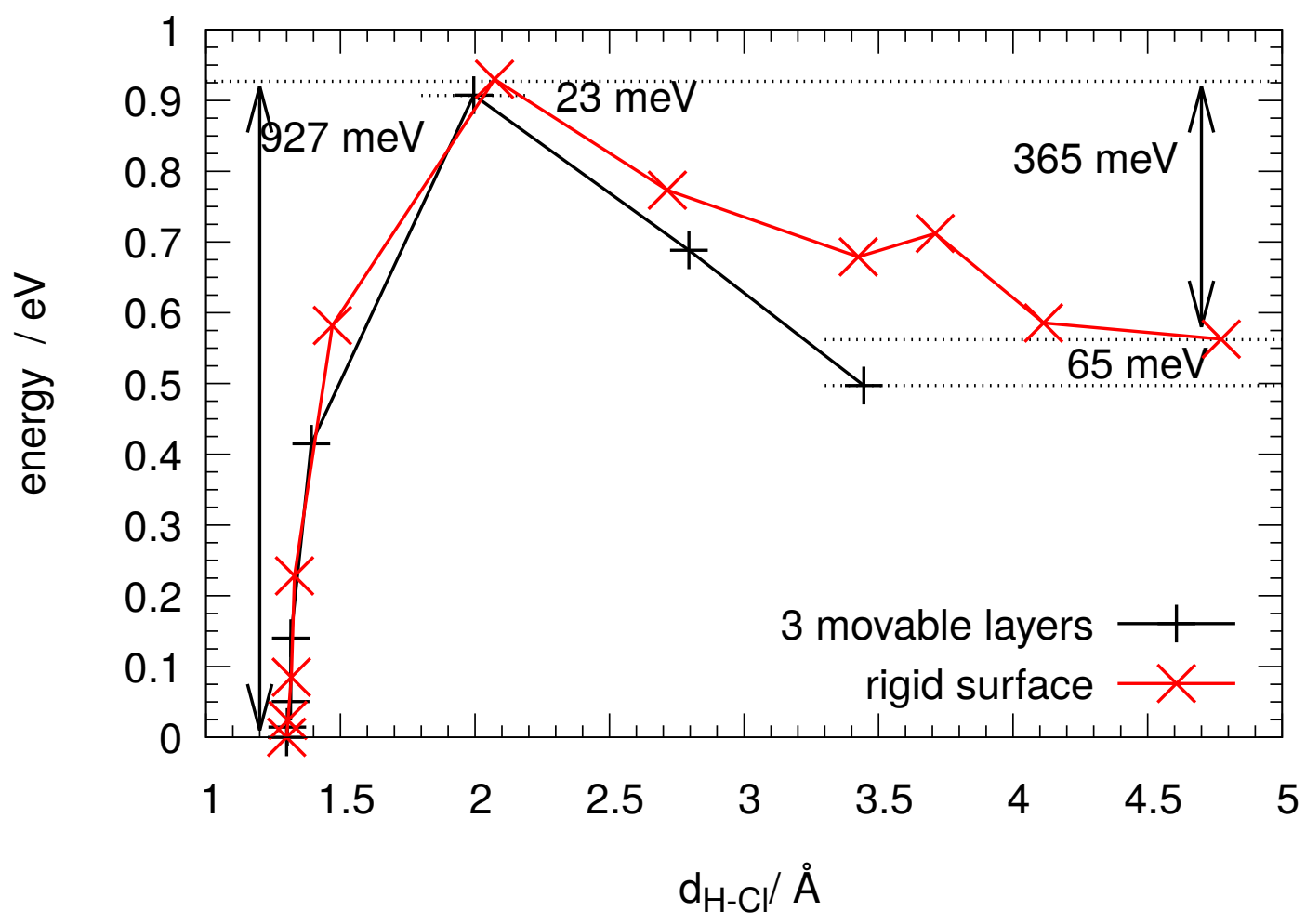

Fig. 22: MEP for the dissociation of $\mathrm{HCl}$ on $\mathrm{Au}(111)$ on a rigid slab (red) and on a slab with movable atoms (black), the reaction coordinate is the $\mathrm{H}-\mathrm{Cl}$ distance $d_{\mathrm{HCl}}$. The arrows indicate the energy differences as given by the numbers.
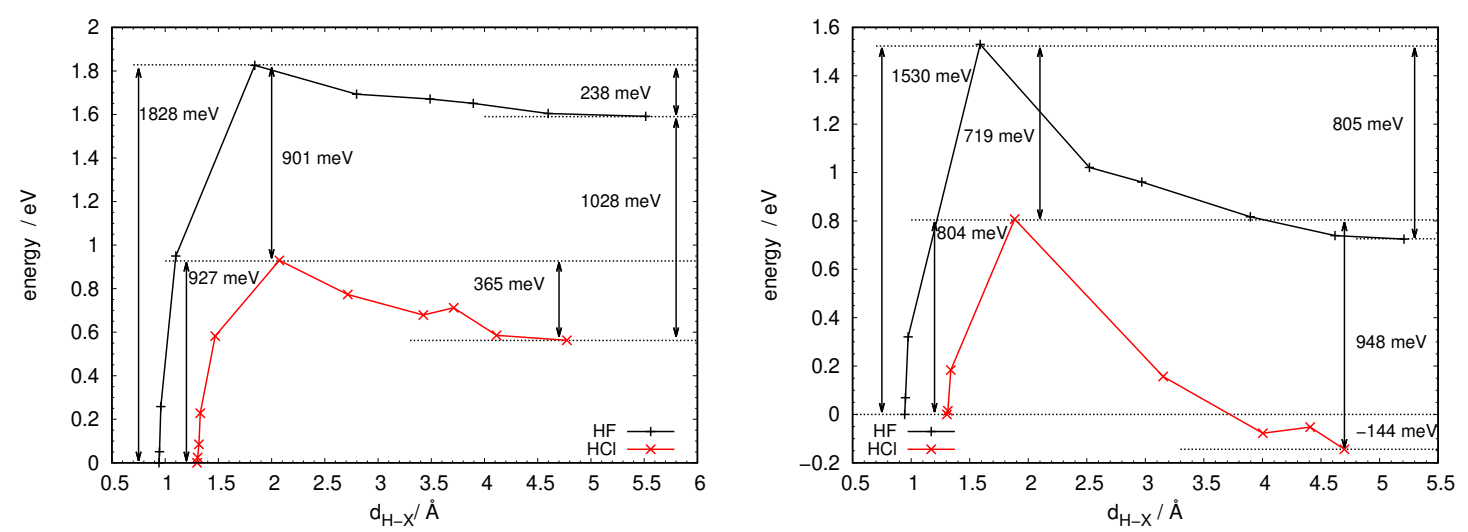

Fig. 23: MEPs for the dissociation of $\mathrm{HCl}$ (red) and HF (black) on Au(111) (left) and Ag(111) (right). The arrows indicate the energy differences given by the numbers.

that the string-method and the FHI-aims code give comparable results to the NEB approach and VASP.

Lately, the group of ZHANG calculated the dissociation barrier for $\mathrm{HCl} / \mathrm{Ag}(111)$ to $0.87 \mathrm{eV}$ (RPBE), $0.61 \mathrm{eV}$ (PW91) and $0.63 \mathrm{eV}$ (PBE) using VASP, respectively. The value for RPBE is $0.07 \mathrm{eV}$ higher than that calculated here $(0.8 \mathrm{eV})$. Furthermore, the dissociation product 
is less stable $(0.2 \mathrm{eV})$ than the reactant, whereas the product is about $0.14 \mathrm{eV}$ more stable than the reactant in the case of calculation done here (see Fig. 23). But the reported configuration of the TS is similar.

However, all DFT calculations show a higher barrier for $\mathrm{HCl} / \mathrm{Au}(111)$ than for $\mathrm{HCl} / \mathrm{Ag}(111)$ and dissociation in the latter case seems to be more likely. But the dissociation barrier in the former case is much lower than it seems to be in experiment [144] and more recent experiments for $\mathrm{HCl} / \mathrm{Ag}(111)$ done by GEWEKE et al. [145] suggests that here dissociative adsorption of the molecule seems to be more likely on the $\mathrm{Ag}(111)$, which shows at least the same trend as the DFT calculations. However, as the discrepancies between experiment and the adiabatic calculations are obvious non-adiabatic effects seems to play an important role in these reactions.

The dissociation barriers for the two molecules on the two surfaces are clearly much lower than the energy necessary to dissociate the molecules in vacuum, which is around $4.47 \mathrm{eV}$ for $\mathrm{HCl}$ and $5.91 \mathrm{eV}$ for $\mathrm{HF}$ [146]. The strong stabilization of the final states in case of $\mathrm{Ag}(111)$ seems to be due to the stronger adsorption of chlorine and hydrogen on this surface. Since the binding of hydrogen on $\mathrm{Au}(111)$ is only $100 \mathrm{meV}$ stronger than its binding to $\operatorname{Ag}(111)[147,148]$, this has to be due to a difference in the chlorine bonding to the surfaces. According to DE LEEEUW et al. the adsorption energy of Cl on Ag(111) is about $1.63 \mathrm{eV}$ [149], and GAO and co-workers reported a value of $0.91 \mathrm{eV} \mathrm{[150]} \mathrm{for} \mathrm{the}$ atom on $\mathrm{Au}(111)$. Hence the $\mathrm{Ag}-\mathrm{Cl}$ bond is about $0.7 \mathrm{eV}$ stronger than the $\mathrm{Cl}$ bond to $\mathrm{Au}$. The adsorption of $\mathrm{F}$ on $\mathrm{Au}(111)$ is about $0.5 \mathrm{eV}$ stronger than that of $\mathrm{Cl}$ according to DFT calculations with the RPBE-functional [147].

\subsection{NO on $\mathrm{Au}(111), \mathrm{Ag}(111)$ and $\mathrm{Cu}(111)$}

For NO, I investigated the MEP for the dissociation on $\mathrm{Au}(111), \operatorname{Ag}(111)$ and $\mathrm{Cu}(111)$. I did these calculations with a $\mathrm{p}(3 \times 3)$ cell with four layers to model the surfaces. The simulation cells were built up by an optimized lattice constant of $4.20 \AA$ for $\mathrm{Au}$ and $\mathrm{Ag}$, and $3.65 \AA$ for $\mathrm{Cu}$ and a width of the vacuum layer of $20 \AA$. Within this model the slab atoms of the three uppermost layers were allowed to move. To find the initial and final states geometry optimizations were done with the adsorbed molecule as well as with both atoms separated from each other. These calculations were again carried out with FHI-aims using the RPBE functional within the spin-polarized GGA. The reciprocal space was sampled with a $4 \times 4 \times 1 \boldsymbol{k}$-point grid as before, and the electronic occupation was described by a Gaussian with a $\sigma$ of $0.2 \mathrm{eV}$. The geometry optimizations were stopped when the forces on all atoms were $<0.01 \mathrm{eV}^{-1}$. The minimum energy structures obtained with light basis sets were confirmed using the tight ones for the 
corresponding species. The following MEP calculations were done with the light settings as well as with the spin-unpolarized approach because the unpaired electron is smeared over the metal surface when the dissociation precedes. All pathways were sampled at least with a minimum of ten images. Again, the string-method within the aimsChain utility was used. The whole pathway is assumed to be optimized when the residual forces in the system are below $0.2 \mathrm{eV}^{-1}$, whereupon the climbing-image-approach was used to optimize the TS configuration so that the forces were smaller than $0.05 \mathrm{eV}^{-1}$.

Tab. 3: Structural parameters for the initial (upper section), TS (middle section) and final (bottom section) configurations and the corresponding energies for $\mathrm{NO}$ on $\mathrm{Au}(111), \operatorname{Ag}(111)$ and $\mathrm{Cu}(111)$. $\theta_{\mathrm{NO}}$ is the angle between the bond distance and the surface normal. $d_{\mathrm{N}-\mathrm{M}}$ and $d_{\mathrm{O}-\mathrm{M}}$ are the distances between nitrogen or the oxygen atoms to the next surface atom. $z_{\mathrm{N}}$ and $z_{\mathbf{O}}$ are the $z$ coordinates of nitrogen and the oxygen atom, respectively. The energy is referenced to the one of the initial state, which value is given below.

\begin{tabular}{lccccccc}
\hline System & $d_{\mathrm{NO}} / \AA$ & $\theta_{\mathrm{NO}} /{ }^{\circ}$ & $z_{\mathrm{N}} / \AA$ & $z_{\mathrm{O}} / \AA$ & $d_{\mathrm{N}-\mathrm{M}} / \AA$ & $d_{\mathrm{O}-\mathrm{M}} / \AA$ & energy/eV \\
\hline $\mathrm{NO} / \mathrm{Au}(111)$ & 1.17 & 52 & 2.54 & 3.25 & 2.37 & 3.14 & $0^{\mathrm{a}}$ \\
$\mathrm{NO} / \mathrm{Ag}(111)$ & 1.18 & 48 & 2.55 & 3.35 & 2.50 & 3.35 & $0^{\mathrm{b}}$ \\
$\mathrm{NO} / \mathrm{Cu}(111)$ & 1.22 & 2 & 1.35 & 2.57 & 2.04 & 2.98 & $0^{\mathrm{c}}$ \\
\hline $\mathrm{NO} / \mathrm{Au}(111)$ & 2.05 & 80 & 1.16 & 1.52 & 2.06 & 2.13 & 3.42 \\
$\mathrm{NO} / \mathrm{Ag}(111)$ & 2.13 & 93 & 1.22 & 1.34 & 2.14 & 2.21 & 3.29 \\
$\mathrm{NO} / \mathrm{Cu}(111)$ & 1.87 & 84 & 1.12 & 1.31 & 1.88 & 1.95 & 1.76 \\
\hline $\mathrm{NO} / \mathrm{Au}(111)$ & 5.14 & 89 & 1.10 & 1.19 & 2.08 & 2.17 & 2.33 \\
$\mathrm{NO} / \mathrm{Ag}(111)$ & 5.15 & 91 & 1.09 & 1.16 & 2.11 & 2.17 & 2.19 \\
$\mathrm{NO} / \mathrm{Cu}(111)$ & 3.97 & 88 & 0.97 & 1.12 & 1.85 & 1.91 & 0.25 \\
\hline $\mathrm{reference}$ energy/ eV: & $\mathrm{a}:-1.9287617662 \times 10^{7}$ & $\mathrm{~b}:-5.273802707 \times 10^{6}$ & \\
$\mathrm{c}:-1.632584769301 \times 10^{6}$ & \multicolumn{7}{c}{} \\
\hline
\end{tabular}

In the case of the geometry optimizations different configurations were optimized to be sure that the true minimum energy structure is found. The initial and final configurations for the three MEP calculations are shown in Fig. 24. For Au(111) and Ag(111) (see Fig. 24a and 24c) the molecule sits on the top site, and the bond axis is tilted to the surface normal between $50-60^{\circ}$. For $\mathrm{Cu}(111)$, however, the initial state looks different; here NO sits parallel to the surface normal on the $f c c$ site (see Fig. 24e), and here the NO bond length is slightly longer than when the molecule is on $\mathrm{Au}(111)$ or $\operatorname{Ag}(111)$. For the final configurations (Fig. 24b,24d,24f) it turns out that the most stable configurations are with both atoms either siting on the $f c c$ or the $h c p$ sites. In case of $\mathrm{Cu}(111)$ both atoms come closer to the surface than in the case of the other metals. An overview about several structural parameters for each initial and final state configuration as well as for the configuration of the TS are given in Tab. 3. 


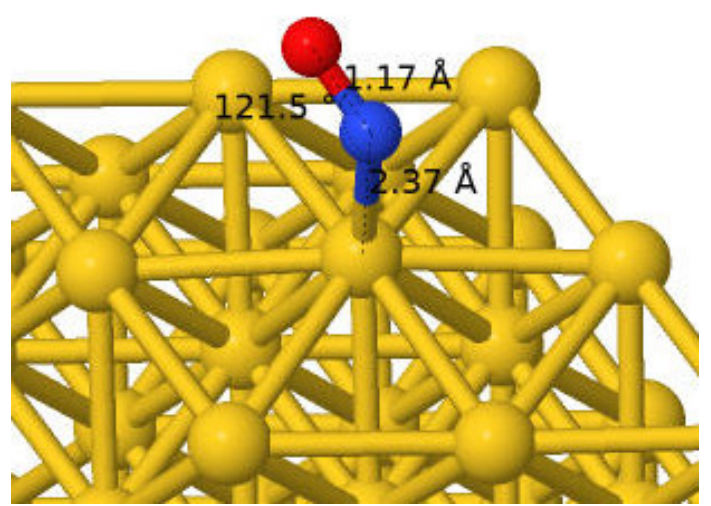

(a) Initial state for NO on Au(111). NO sits on top site.

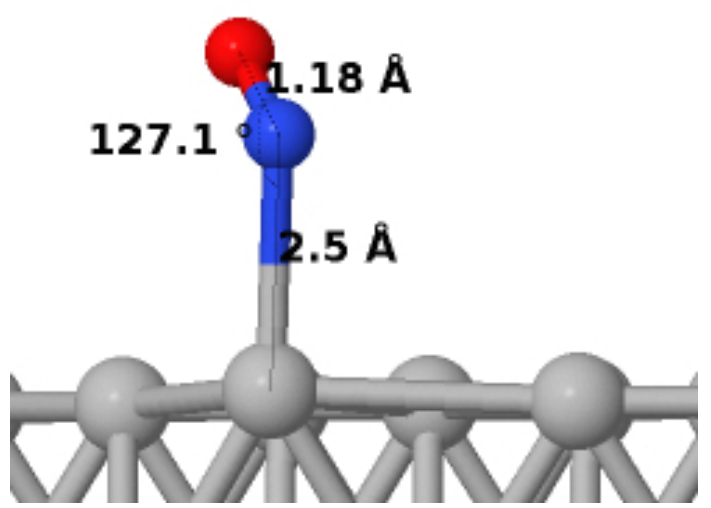

(c) Initial state for NO on $\mathrm{Ag}(111)$. NO is on top site.

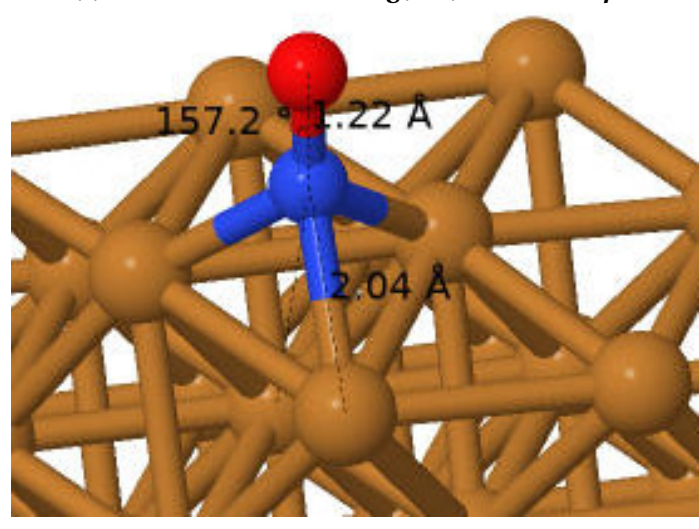

(e) Initial state for NO on $\mathrm{Cu}(111)$. NO sits on $f c c$ site.

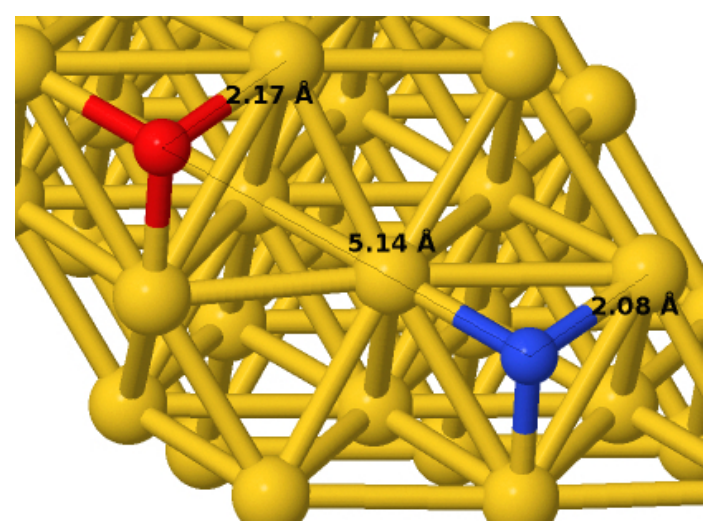

(b) Final state for NO on $\mathrm{Au}(111) . \mathrm{N}$ and $\mathrm{O}$ sit both on $f c c$ sites.

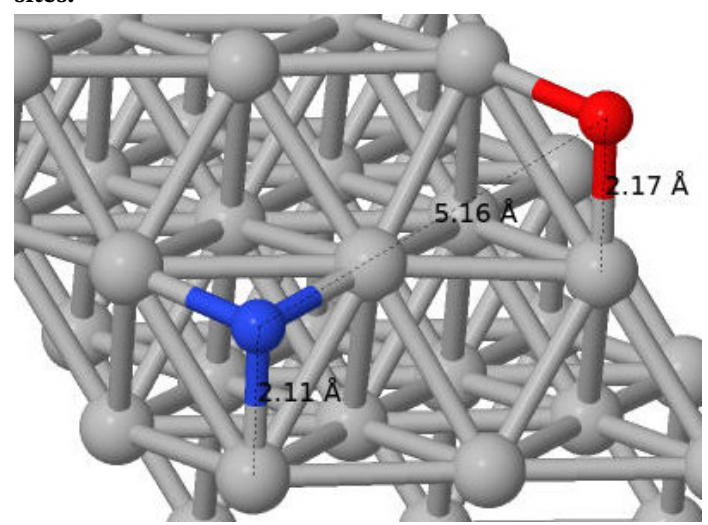

(d) Final state for NO on $\mathrm{Ag}(111)$. $\mathrm{N}$ and $\mathrm{O}$ sit both on $f c c$ sites.

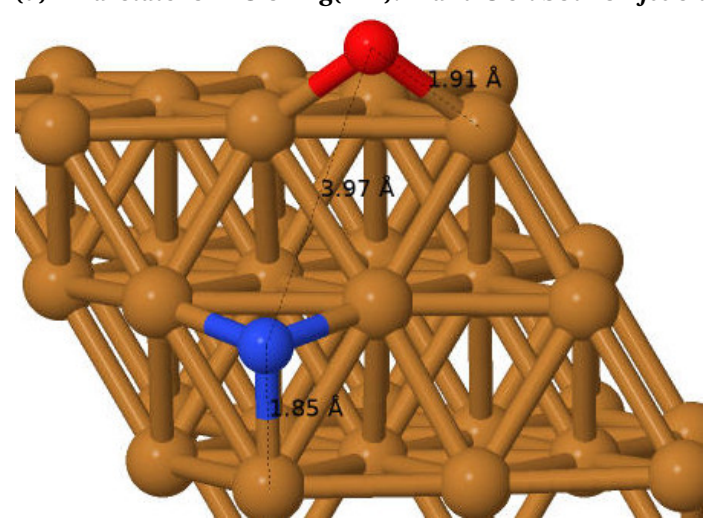

(f) Final state for NO on $\mathrm{Cu}(111) . \mathrm{N}$ sits on the $f c c$ and $\mathrm{O}$ sits on the hcp site.

Fig. 24: Initial (a, c, e) and final states (b, d, f) for the dissociation of NO on $\mathrm{Au}(111), \operatorname{Ag}(111)$ and $\mathrm{Cu}(111)$. N, O, Au, Ag and $\mathrm{Cu}$ atoms are represented by blue, red, dark yellow, gray and brown spheres, respectively. The numbers and lines are indicating distances and angles between corresponding atoms. 


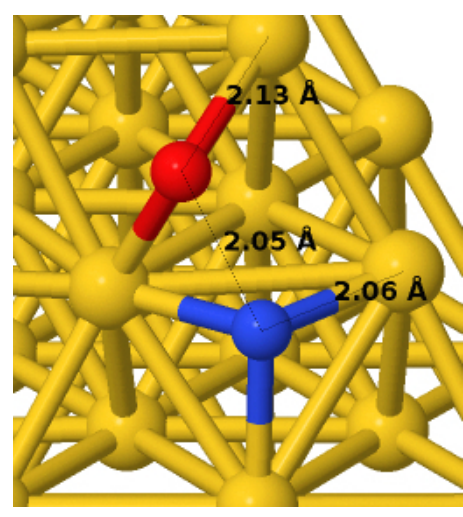

(a) TS configuration for NO on Au(111), where dark yellow spheres represent the gold atoms.

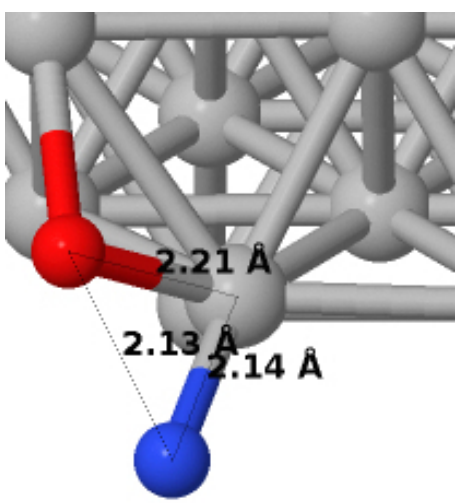

(b) TS configuration for NO on $\mathrm{Ag}(111)$, where gray spheres represent the silver atoms.

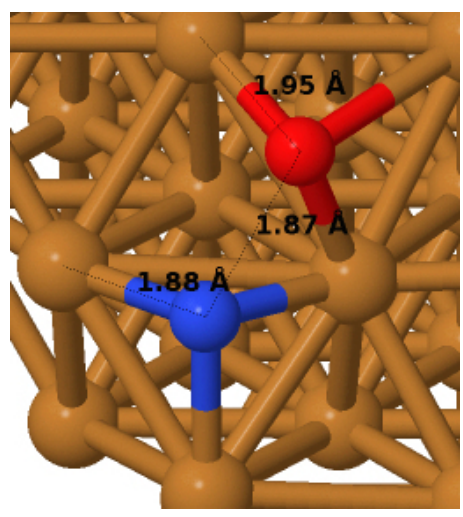

(c) TS configuration for NO on $\mathrm{Cu}(111)$, where brown spheres represent the copper atoms.

Fig. 25: Configurations of the transition states for the dissociation of NO on $\operatorname{Au}(111)(a), \operatorname{Ag}(111)$ (b) and $\mathrm{Cu}(111)$ (c), respectively. The $\mathrm{N}$ and $\mathrm{O}$ atoms are represented by blue and red spheres, respectively. Dashed lines and numbers indicate atom distances.

The transition state configurations (see Fig. 25) for the dissociation do not show strong differences for the three surfaces. $\mathrm{N}$ sits on a hallow site and $\mathrm{O}$ bridges two top atoms in each case. For $\mathrm{NO}$ on $\mathrm{Cu}(111)$ the distance between $\mathrm{N}$ and $\mathrm{O}$ is around $30 \%$ shorter than on the more noble surfaces. Further the $\mathrm{N}$ atom as well as $\mathrm{O}$ comes closer to the surface in case of $\mathrm{Cu}(111)$. All three TS configurations look more like the product configurations and so Polanyi's rules suggest a higher probability of product formation via vibrational excitation for this kind of TS configuration.

The MEPs for all three dissociation reactions, as shown in Fig. 26, reveal that the energy barriers for the reaction on $\mathrm{Au}(111)$ and $\mathrm{Ag}(111)$ are similar to each other, that means $3.42 \mathrm{eV}$ and $3.27 \mathrm{eV}$, respectively. To break the NO bond on $\mathrm{Cu}(111)$ much less energy $(1.76 \mathrm{eV})$ is needed than on the surfaces of higher row elements. So the shorter NO bond distance $d_{\mathrm{NO}}$ of $1.87 \mathrm{eV}$ in the case of $\mathrm{Cu}(111)$ comes along with a drastic reduction of the energy barrier, thus the dissociation of the diatomic is clearly more favorable on this surface, which is more reactive, than on $\mathrm{Au}(111)$ and $\mathrm{Ag}(111)$. The reactions on the different metals are all endothermic so the product configurations are energetically higher and so less stable than those of the initial ones. Also, in this aspect is the reaction on $\mathrm{Cu}(111)$ more likely because the energy difference between the two states is just about $0.25 \mathrm{eV}$ which is much smaller than the difference of $\sim 1.40 \mathrm{eV}$ on $\mathrm{Au}(111)$ and $\mathrm{Ag}(111)$. However, these results show that the dissociation reaction on all investigated surfaces is thermodynamically not favorable when the entropic part is neglected as the temperature is zero. The TS configurations look similar to those found by GADJOŠ et al. calculated with VASP in the NEB approach and with the PW91 functional [151]. The energy barriers for dissociation are also in qualitative agreement with those calculated with which is known to give lower barriers than the RPBE functional used here. 


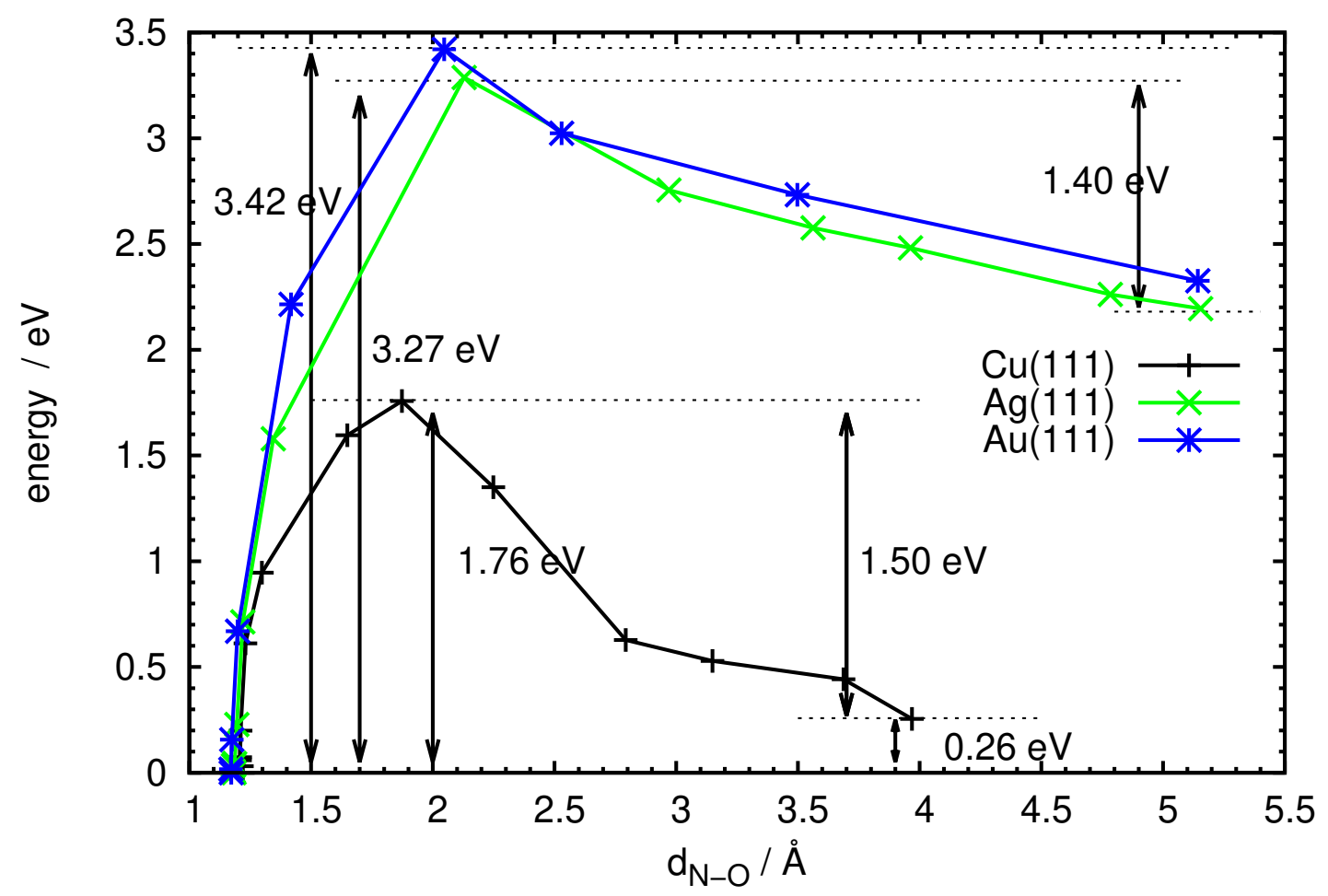

Fig. 26: MEPs for NO dissociation on $\mathrm{Cu}(111)$ (black crosses), $\mathrm{Ag}(111)$ (green crosses) and $\mathrm{Au}(111)$ (blue stars). As reaction coordinate the $\mathrm{N}-\mathrm{O}$ distance $d_{\mathrm{NO}}$ is used. The numbers give the energy differences between different states of the reaction process, which are indicated by black dotted lines and black arrows. 


\subsection{CO oxidation on $\operatorname{Pt}(111)$}

The oxidation of $\mathrm{CO}$ on platinum is one of the most investigated reactions in surface science due to its important role in the combustion of fossil fuels in car engines. The MEP calculations shown in this section are among other things done to theoretically support new experiments by NEUGEBOHREN et al. on this reaction [38]. These experiments used the ion or velocity map imaging-technique to shed light on this oxidation on this catalytic surface. The experiments show that defects, like kinks and steps on the surface, enhance the catalytic activity and hence the rate of the oxidation. The influence of defects on this process was not clear and the whole mechanism of the oxidation is under debate, too. However, the surface scattering experiments on $\mathrm{Pt}(111)$ and $\mathrm{Pt}(332)$ applying the velocity map imaging reveal that $\mathrm{CO}_{2}$ formation is faster on the (332)-surface which has a larger step density, thus more defects than the (111)surface. The experiment reveals two reaction channels for CO-oxidation; one leads to a hyper-thermal $\mathrm{CO}_{2}$ and one to a thermal product. The first channel is more dominant on the flat (111)-surface, whereas the second channel dominates on $\operatorname{Pt}(332)$ and the hyper-thermal channel vanishes.

The MEP calculations done here are just describing the situation on the flat (111)surface only. They comprise the simulation of a high ( $0.25 \mathrm{ML})$ and a low (0.11 ML) coverage of $\mathrm{CO}$ and $\mathrm{O}$ by employing a $\mathrm{p}(2 \times 2)$ and a $\mathrm{p}(3 \times 3)$ cell with 4 layers. In the $z$-direction a vacuum distance of $20.0 \AA$ was used. The slab atoms were kept fixed at their equilibrium positions with an optimized lattice constant of $4.00 \AA$. For the search of the TS FHI-aims with the aimsChain utility was used. To find the initial and final configurations of the MEP geometry optimizations were done. The latter were carried out with a $\mathrm{CO}_{2}$ molecule about $4 \AA$ above the surface, for the former the situation was a bit more complicated. Here different configurations of $\mathrm{CO}$ and $\mathrm{O}$ on the surface are possible, especially for the larger $\mathrm{p}(3 \times 3)$ cell. In this case several geometry optimizations were made for which either $\mathrm{CO}$ or $\mathrm{O}$ were initially placed on different sites of the surface. From these calculations the one having the smallest energy was used as the initial configuration. For all these geometry optimizations the following parameters were used:

(i) the RPBE functional including van-der-Waals (vdW) corrections (TKATCHENKO and SCHEFFLER [152]) within the spin-unpolarized approach was used.

(ii) the reciprocal space was sampled by a $4 \times 4 \times 1 \boldsymbol{k}$-point grid.

(iii) the electronic occupation was modeled by a Gaussian function with a width $\sigma=$ $0.2 \mathrm{eV}$. 
(iv) the wave function basis sets were described by light-settings.

(v) the geometry optimizations were stopped when the forces acting on an atom were smaller than $0.01 \mathrm{eV} \AA^{-1}$.

The geometry optimized structures are presented in Fig. 27. The minimum energy structures of both cells for the separated $\mathrm{CO}$ and $\mathrm{O}$ are similar, that means the CO sits perpendicular on the top site and the $\mathrm{O}$ atom is placed on the fcc site, see Figs. 27a and 27c. In the case of the formed $\mathrm{CO}_{2}$ the energetic structure minimization shows that the molecule is about $4 \AA$ away from the surface perpendicular to its normal. The molecule has a linear configuration, and both $\mathrm{O}$ atoms have a distance of $1.17 \AA$ to the $\mathrm{C}$ atom.

Tab. 4: Structural parameters for the initial (upper section), TS (middle section) and final (bottom section) configurations and the corresponding energies for the $\mathrm{CO}$ oxidation for in different sized cells, representing different coverage. $\theta_{\mathrm{CO}}$ is the angle between the bond distance and the surface normal. $d_{\mathrm{C}-\mathrm{M}}$ and $d_{\mathrm{O}_{\text {ads }} \mathrm{M}}$ are the distances between the $\mathrm{C}$ and the adsorbed oxygen atom to the next surface atom, respectively. $z_{\mathrm{C}}$ and $z_{\mathrm{O}_{\text {ads }}}$ are the $z$ coordinates of $\mathrm{C}$ and the adsorbed $\mathrm{O}$ atom, respectively. The energy is referenced to the one of the initial state, which value is given below.

\begin{tabular}{lccccccc}
\hline System & $d_{\mathrm{C}-\mathrm{O}_{\mathrm{ads}}} / \AA$ & $\theta_{\mathrm{CO}} /{ }^{\circ}$ & $z_{\mathrm{C}} / \AA$ & $z_{\mathrm{O}_{\mathrm{ads}}} / \AA$ & $d_{\mathrm{C}-\mathrm{M}} / \AA$ & $d_{\mathrm{O}_{\mathrm{ads}} \mathrm{M}} / \AA$ & energy/ eV \\
\hline $\mathrm{p}(3 \times 3)$ & 4.36 & 0 & 1.84 & 1.24 & 1.86 & 2.05 & $0^{\mathrm{a}}$ \\
$\mathrm{p}(2 \times 2)$ & 3.32 & 0 & 1.88 & 1.26 & 1.88 & 2.06 & $0^{\mathrm{b}}$ \\
$\mathrm{p}(4 \times 4)$ & 3.32 & 4 & 1.87 & 1.26 & 1.87 & 2.06 & $0^{\mathrm{c}}$ \\
\hline $\mathrm{p}(3 \times 3)$ & 2.03 & 75 & 1.87 & 3.02 & 1.93 & 3.05 & 0.775 \\
$\mathrm{p}(2 \times 2)$ & 1.97 & 93 & 1.87 & 1.52 & 1.94 & 2.08 & 0.881 \\
$\mathrm{p}(4 \times 4)$ & 1.9 & 12 & 1.86 & 3.01 & 1.88 & 2.09 & 0.843 \\
\hline $\mathrm{p}(3 \times 3)$ & 1.18 & 89 & 3.72 & 3.70 & 4.03 & 3.81 & -1.134 \\
$\mathrm{p}(2 \times 2)$ & 1.18 & 91 & 3.78 & 3.76 & 3.98 & 3.78 & -0.895 \\
$\mathrm{p}(4 \times 4)$ & 1.18 & 90 & 3.75 & 3.74 & 3.87 & 3.92 & -0.640 \\
\hline reference energy $/ \mathrm{eV}:$ & $\mathrm{a}:-1.8664169641 \times 10^{7}$ & & & \\
$\mathrm{~b}:-8.2980410884 \times 10^{6}$ & $\mathrm{c}:-1.6606347512 \times 10^{7}$ & & & \\
\hline
\end{tabular}

The determined minimum energy configurations of $\mathrm{CO}$ and $\mathrm{O}$ on the surface as well as $\mathrm{CO}_{2}$ away from the $\mathrm{Pt}(111)$ surface were used as initial and final configurations to start the MEP calculations. The same control parameters were used as for the calculations described above. The MEPs in the two cells were sampled with different numbers of images as it turned out that for the larger cell a large number of images (17) was necessary to actually find the TS for the reaction in this cell. As for the calculations in the previous section the string method with a force criterion of $0.2 \mathrm{eVA}^{-1}$ for the whole path was used, and after reaching this a second criterion for the climbing image of $0.05 \mathrm{eV}^{-1}$ was applied to ensure the finding the TS configuration. 


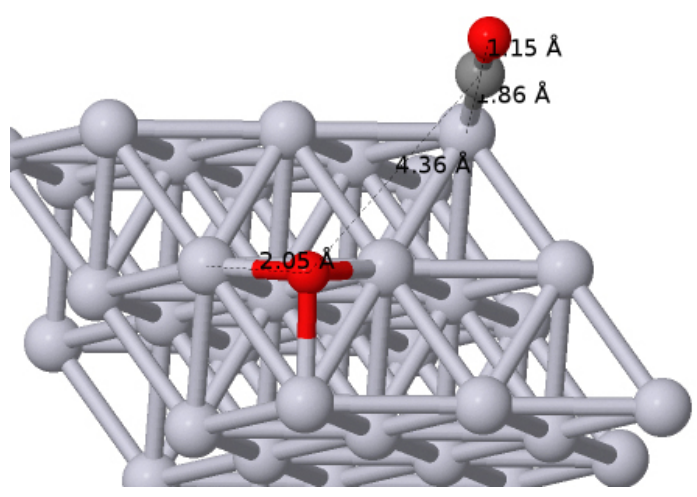

(a) Initial state in the $\mathrm{p}(3 \times 3)$ cell.

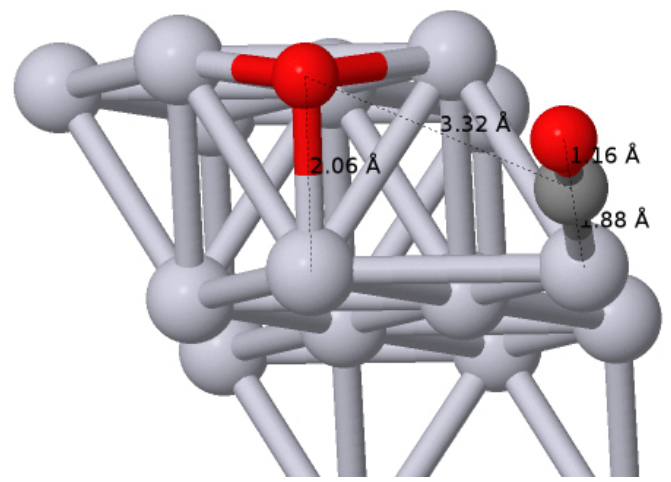

(c) Initial state in the $\mathbf{p}(2 \times 2)$ cell.

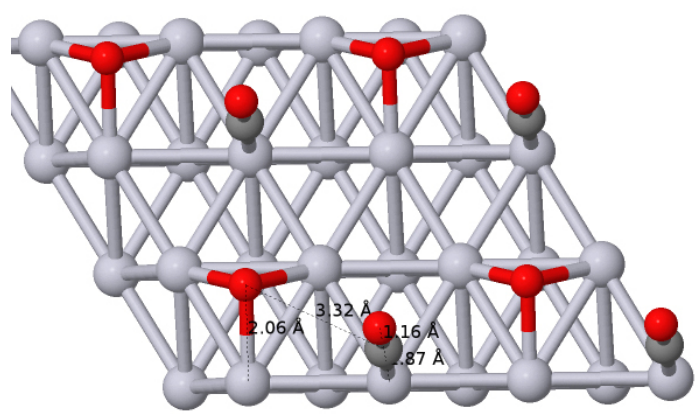

(e) Initial state in the $\mathrm{p}(4 \times 4)$ cell.

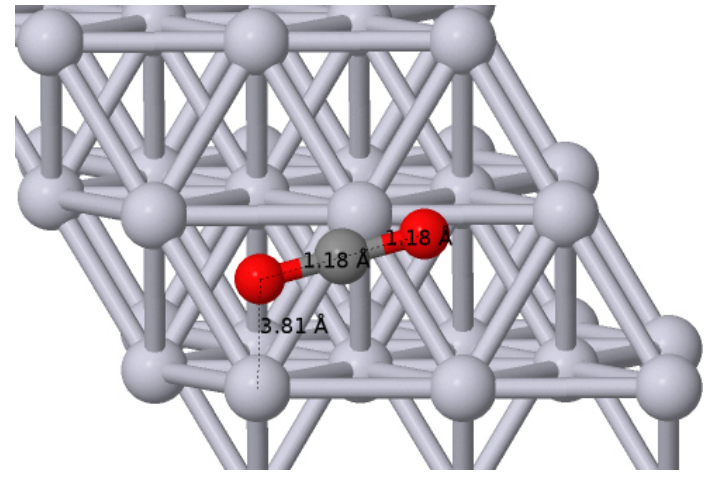

(b) Final state in the $\mathbf{p}(3 \times 3)$ cell.

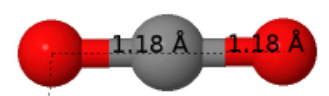

$3.78 \AA$

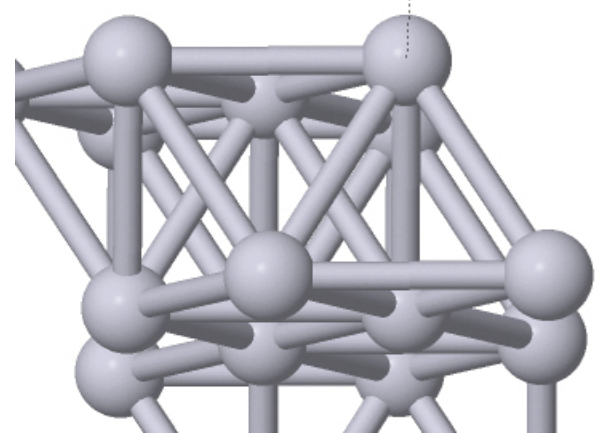

(d) Final state in the $\mathrm{p}(2 \times 2)$ cell.

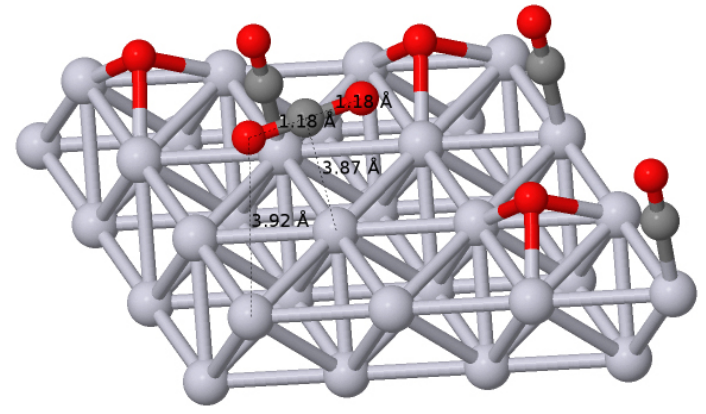

(f) Final state in the $p(4 \times 4)$ cell.

Fig. 27: Initial (a,c,e) and final states (b,d,f) for the MEP of CO oxidation in the $p(3 \times 3)$ (top), in the $p(2 \times 2)($ middle) and in the $p(4 \times 4)$ cell (bottom panel), respectively. $O, C$ and $P t$ atoms are represented by red, dark and light gray spheres, respectively. The numbers and lines indicate distances and angles between corresponding atoms. 


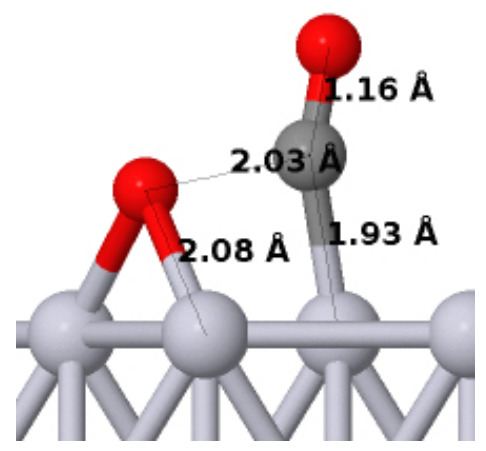

(a) TS configuration found for in a $\mathbf{p}(3 \times 3)$ cell.

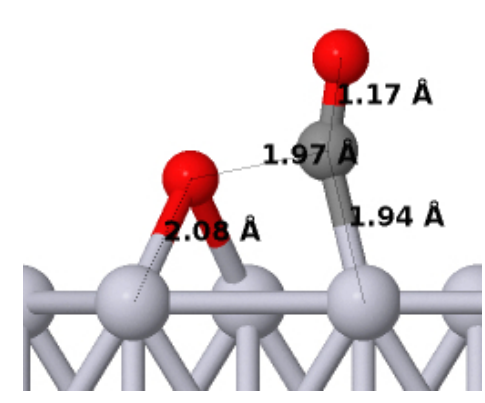

(b) TS configuration found for in a p $(2 \times 2)$ cell.

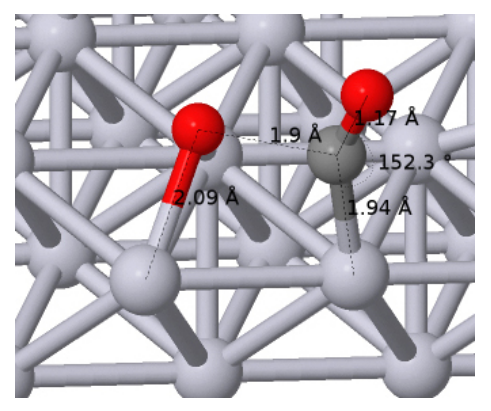

(c) TS configuration found for a p $(4 \times 4)$ cell.

Fig. 28: Transition state configurations for $C O$ oxidation on $\operatorname{Pt}(111)$ in a p(3 $\times 3)(a), a p(2 \times 2)$ cell (b) and a p(4×4) (c). C, $\mathrm{O}$ and $\mathrm{Pt}$ atoms are represented by dark gray, red and light gray spheres, respectively. Numbers indicate distances between atoms.

For both cells the TS configurations are very similar, thus the distances between the atoms and their arrangement on the surface show no large differences. This can be seen in Fig. 28. At the TS configuration CO is slightly tilted with respect to the surface normal, and it does not sit on top of a Pt atom. The adsorbed $\mathrm{O}$ atom bridges two surface atoms and is about $2 \AA$ away from the $\mathrm{C}$ atom, and the molecule is tilted so that the $\mathrm{O}$ evades the adsorbed $\mathrm{O}$ atom. Since the configuration reminds us more of the employed reactants, the TS can be classified as a early one. Therefore, product formation is more promoted by increasing translational energy than vibrational energy. As Polanyi's rules further predict the formed product should be vibrationally excited.

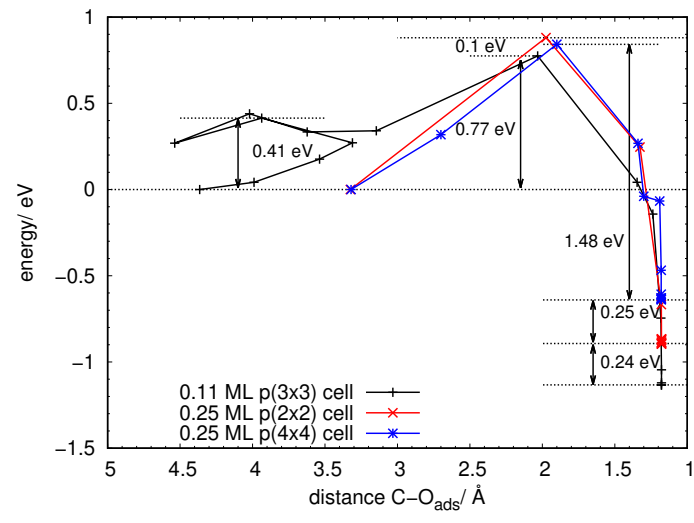

Fig. 29: The MEPs of the CO oxidation on $\operatorname{Pt}(111)$ for the $p(2 \times 2), p(3 \times 3)$ and $p(4 \times 4)$ cells are presented by red, black and blue crosses respectively. The dotted lines and the arrows indicate the energy difference between states whose value is given by the numbers in $\mathrm{eV}$. The distance between $\mathrm{C}$ and adsorbed $\mathrm{O}$ atom is shown as reaction coordinate.

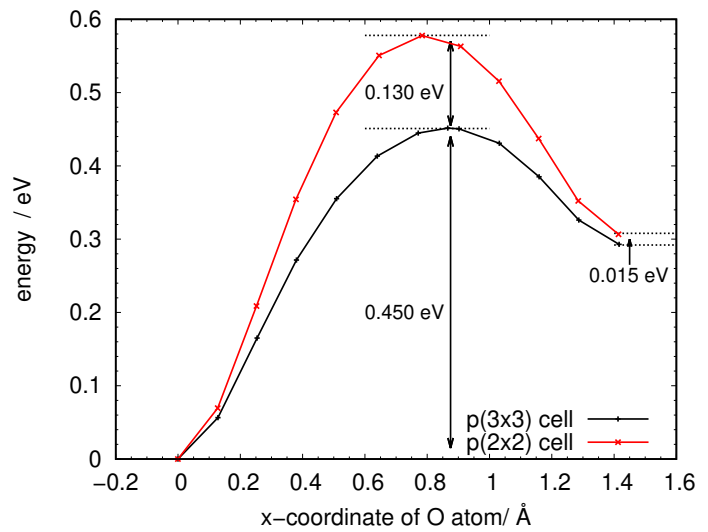

Fig. 30: The MEPs of the $O$ diffusion on $\operatorname{Pt}(111)$ from $f c c$ - to $h c p$ site for the $p(3 \times 3)$ and $p(2 \times 2)$ cells are presented by black and green crosses, respectively. The dotted lines and the arrows indicate the energy difference between states whose value is given by the numbers in $\mathrm{eV}$. 
The energetics of the two pathways is shown in Fig. 29 (black and red lines). Here the two paths show that the barrier heights are in a qualitative agreement and thus the $p(2 \times 2)$ cell shows a $0.11 \mathrm{eV}$ higher barrier with respect to the energies of the reactants than the larger cell. This seems to be attributed to lateral interactions between periodic images. Although the initial configurations are also similar the MEPs are not. For the $\mathrm{p}(3 \times 3)$ cell there are more configurations until the reactants reach the TS than in case of the high coverage where the reactants are immediately forming this state. In the case of the $\mathrm{p}(3 \times 3)$ cell the smaller coverage makes it possible that the $\mathrm{O}$ atom can move from one hollow site to another. This diffusion of the $\mathrm{O}$ atom from the $f c c$ to the $h c p$ site is attended by an increase of the energy of about $0.4 \mathrm{eV}$ (see black line in Fig. 29). Furthermore, the $\mathrm{O}$ atom diffusion leads in the interim to a larger distance of the reactants to each other. After this the reactants come closer together again and the TS is formed. The final states differ in energy about $0.15 \mathrm{eV}$ which can be seen as some long range interaction between images. So one can say that an $\mathrm{O}$ atom adsorbed at the $h c p$ site is more active than an atom sitting on $f c c$ site because when starting the reaction from the former site the reaction barrier is about $50 \%$ lower than from the latter.

In principle, it is not realistic to use a $\mathrm{p}(2 \times 2)$ cell to model the $0.25 \mathrm{ML}$ coverage, because due to the pbc it models the reaction such that every $\mathrm{O}$ and $\mathrm{CO}$ which sit on the surface react to $\mathrm{CO}_{2}$. So to model the $0.25 \mathrm{ML}$ coverage more realistically I did calculations with a $\mathrm{p}(4 \times 4)$ cell, where I placed $4 \mathrm{O}$ and $4 \mathrm{CO}$. Within this just one $\mathrm{O}$ and $\mathrm{CO}$ were allowed to move; all other atoms were kept fixed at their equilibrium positions. The surface was modeled with just two layers to save computational time. The initial and final states for this cell are shown in Fig. 27e and Fig. 27f. Both states look similar to those of the $p(2 \times 2)$ cell. The TS configuration for this cell is presented in Fig. 28c. There are only slight differences to configurations in the other cells, that means the distance between $\mathrm{C}$ and the adsorbed $\mathrm{O}$ atom is $1.9 \AA$, thus about $0.1 \AA$ shorter than the distance for the other cases. The found MEP is also represented in Fig 29 by blue crosses. It shows that the barrier height is nearly the same as for the $\mathrm{p}(2 \times 2)$ cell, but the product state for the larger cell is about $0.25 \mathrm{eV}$ less stable than the one of the smaller cell. This differences in energy maybe attributed to long-range interactions between images of the molecule in the smaller cell.

Furthermore, the first barrier of $0.41 \mathrm{eV}$ in the case of the $\mathrm{p}(3 \times 3)$ cell (black line in Fig. 29) seems mainly due to diffusion of the adsorbed $O$ from one hollow site to the other. This can be concluded from Fig. 31. Here the initial state is compared with a configuration after the first barrier. As said before in the initial configuration the $\mathrm{O}$ atom sits on the $f c c$ site and the CO sits on top site (see Fig. 31a), whereas in the other 
configuration (Fig. 31b), when the reaction precedes, O sits on the hcp site and CO has moved to another top site.

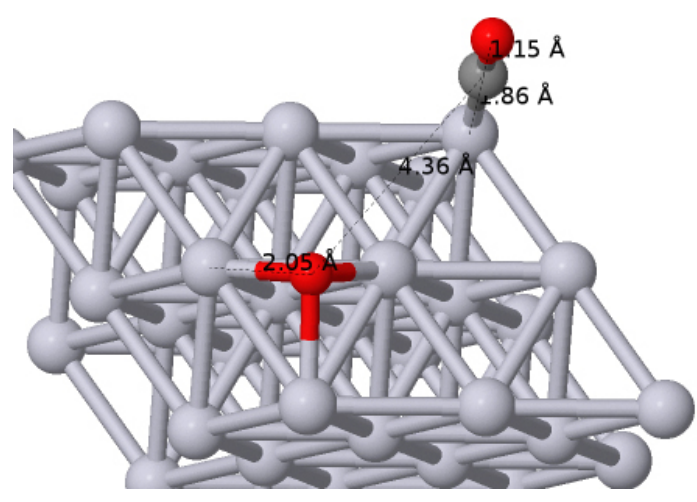

(a) Initial state for $\mathrm{CO}$ oxidation in the $\mathrm{p}(3 \times 3)$ cell

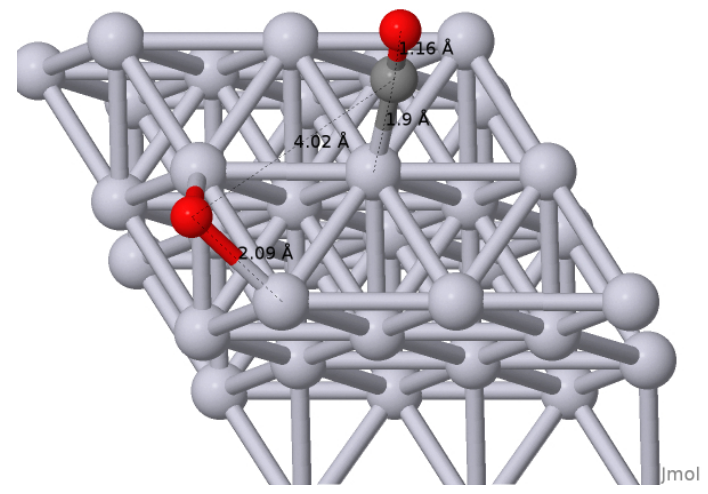

(b) Configuration after the first barrier in the MEP was passed.

Fig. 31: Configurations for the initial state (a) and a configuration after the first barrier (b) in the MEP for the $p(3 \times 3)$ cell. The $C, O$ and $P t$ atoms are represented by dark gray, red and light gray spheres, respectively, and the numbers indicate distances between atoms.

To test this assumption the MEP of this reaction in both cells was calculated and reveals a barrier height of $0.41 \mathrm{eV}$ for the reaction pathway in the case of the lower coverage $(\mathrm{p}(3 \times 3)$ cell $)$ and reveals a significant higher barrier of $0.55 \mathrm{eV}$ for the higher O coverage $(p(2 \times 2)$ cell $)$ of the surface. This is shown in Fig. 30 . So the large difference between the cells for the barrier heights may be due to interactions between periodic images, but in the case of a single atom interactions above a range of $5.5 \AA$ must be responsible for this. One could further investigate this by using a $\mathrm{p}(4 \times 4)$ cell with one atom as well as with $4 \mathrm{O}$ atoms, where one does the diffusion reaction with one atom and keeps the three other atoms fixed at their initial site. With the first case we could see if the barrier is further lowered for a even lower $O$ coverage. With the second case one can model the diffusion of the atom for a coverage of $0.25 \mathrm{ML}$ more realistically, i.e. the movement of the $\mathrm{O}$ atom is independent from movement of the other atoms as done for the oxidation of CO. In the case of the $\mathrm{p}(2 \times 2)$ cell, however, the $\mathrm{O}$ atom sees just its images doing the same motion. This is in principle a model for the case in which a large fraction of $\mathrm{O}$ atoms sit on $f c c$ sites and moves simultaneously to the $h c p$ sites, although this seems unlikely.

However, we can conclude that this first barrier in case of the $p(3 \times 3)$ cell is clearly due to the diffusion of the $\mathrm{O}$ atom and thus the reaction between $\mathrm{CO}$ and $\mathrm{O}$ adsorbed at $\mathrm{a}$ $h c p$ site has a smaller barrier than those with an O adsorbed at the $f c c$ site. This means that in the former case the $\mathrm{O}$ sits on an activated site.

To to gain further insight into the ongoing process and to determine the final translational energy of the product, $\mathrm{CO}_{2}$, further theoretical efforts were done. I started 
AIMD simulations with FHI-aims, where I placed the reactants, $\mathrm{CO}$ and $\mathrm{O}$, close to the configuration of the TS found by the MEP calculations. Then I randomly moved both reactants up to $0.1 \AA$ away from their positions of the TS configuration. The velocity vectors of the three atoms were determined in the following way: their direction is set towards the product state of the MEP and the length of the vector corresponds to a translational energy of $0.3 \mathrm{eV}$. This energy was chosen so that the reactants reach the product state in a relatively short time of about $0.5 \mathrm{ps}$. The simulations were carried out in a $\mathrm{p}(3 \times 3)$ slab with 4 layers, whereby the bottom layer was kept fixed and the velocities of the atoms in the 3 top layers were set to zero, respectively, which means that the slab temperature corresponds to $0 \mathrm{~K}$. The slab atoms were initially placed at their positions of the TS configuration found in MEP calculation, as described above.

Furthermore, the simulations were controlled by the following parameters: the RPBE functional plus vdW-corrections to account for the exchange-correlation as well as for the long-range interaction. The electronic occupation was described by a Gaussian and a width of $0.2 \mathrm{eV}$, and the reciprocal space was sampled by a $4 \times 4 \times 1 \boldsymbol{k}$-point grid. The basis sets were defined by light settings. A time step of $0.5 \mathrm{fs}$ was used, and to stop the $s c f$-cycle criteria of $1 \times 10^{-6}$ for the total energy and $1 \times 10^{-5}$ for the forces were used. The simulations were stopped after 1 ps or when the distance of the COM of molecule to the slab was larger than $4.5 \AA$.

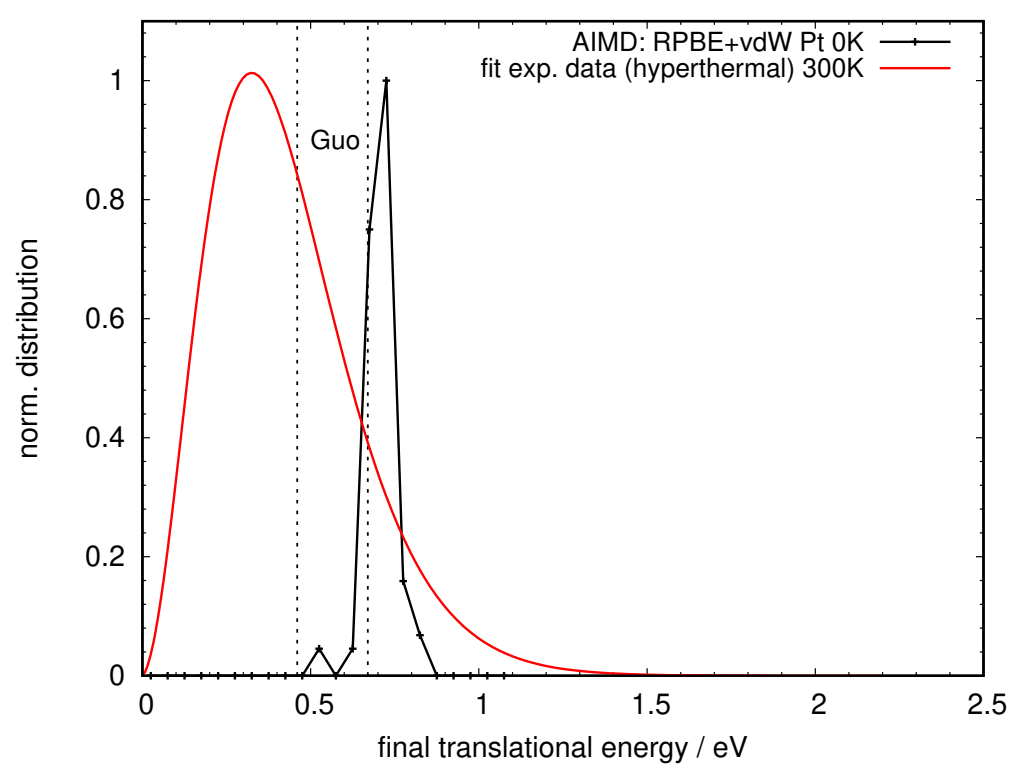

Fig. 32: Final translational energy distribution of the formed $\mathrm{CO}_{2}$ (normalized to maximum signal) of the experimentally observed hyper-thermal channel (red line) and the AIMD simulations (black line). The dotted lines indicate the range of simulations done with PW91 (from private communications with Hua Guo). 


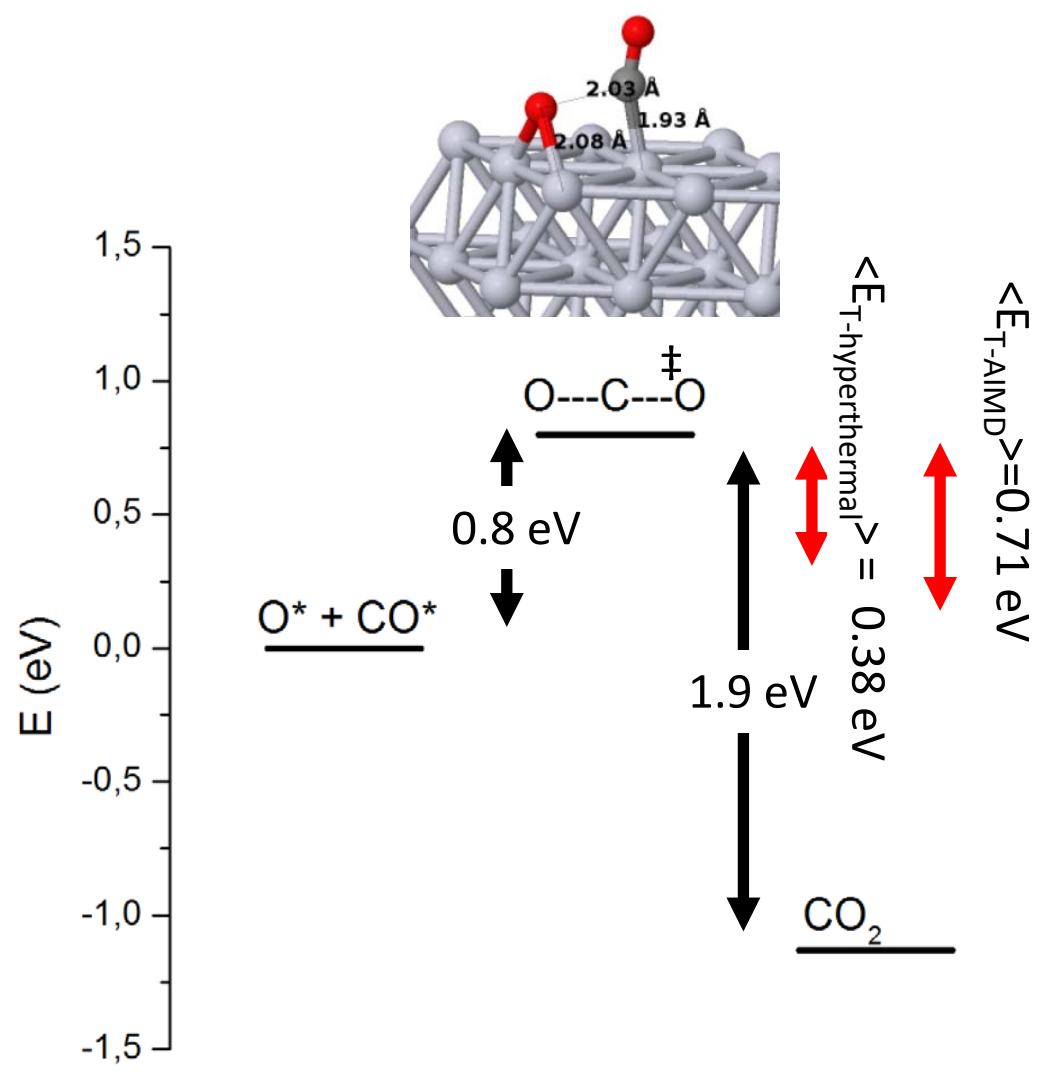

Fig. 33: Scheme of the MEP including the TS configuration. The barrier height and the energy release of the MEP are shown by black arrows, and the mean final translational energies from experiment and AIMD are shown by red arrows. 
The final translational energy distribution of the formed $\mathrm{CO}_{2}$ is shown in Fig. 32. The distribution is relatively narrow and has a mean value of about $0.71 \mathrm{eV}$, which agrees with the calculations done with PW91 by ZHOU et al. [153]. It deviates from the experimental data, which show a broader behavior and has a maximum at about $0.320 \mathrm{eV}$. This may be due to the difference in the surface temperature $(0 \mathrm{~K}$ for the simulations and $300 \mathrm{~K}$ for experiment) and also due to the initial translational energy of the reactants in the case of the simulations. Furthermore, since the RPBE functional is known to lead to overestimate reaction barriers, it may be the case that the translational energy of the product obtained from this TS is also overestimated and therefore higher than in the experiment. A further explanation for the differences between experiment and simulation is that in the former molecular energy may also go into electronic dofs of the surface, i.e. ehp excitation. This is not possible in the adiabatic simulations. The width of the distribution of the experiment is much broader than the one of the simulations, this suggest that in the latter case an important channel to transfer translational energy is missing. This channel maybe the energy transfer to ehps but also to the phonon of the surface. Since the simulations of ZHOU et al. [153], done with a slab equilibrated to $600 \mathrm{~K}$ show a broader distribution, than those calculated here, the transfer to the phonons of the slab is important. Therefore, non-adiabatic effects may only play a minor role in the energy transfer.

The final translational energy of the formed $\mathrm{CO}_{2}$ is much smaller than the total energy release of the reaction as seen in Fig. 33). Here, the total energy release, and so the total energy of $\mathrm{CO}_{2}$ is $1.9 \mathrm{eV}$, whereas the mean final translational energies of the molecule are $0.38 \mathrm{eV}$ (experiment hyperthermal channel) and $0.71 \mathrm{eV}$ (AIMD). Since the reaction has an early TS (see Fig. 33) a lot of the energy, released during the reaction, goes into rotational and especially into vibrational dofs, and just a smaller amount goes into translational excitation of CO. Therefore, this picture is in agreement with the experimental and theoretical results.

\subsection{Conclusions}

The MEPs found for the investigated dissociation reactions show that all TS configurations resemble the reaction products; therefore, we can classify the reactions as late barrier reactions. Hence, according to Polanyi's rules, vibrational excitation of the reactant should promote the reactions, and the products should be translationally excited. The dissociation barrier for $\mathrm{HCl}$ is much lower than the one for $\mathrm{HF}$ on either $\mathrm{Au}(111)$ or $\mathrm{Ag}(111)$. Furthermore, the reaction products are more stable in the case of $\mathrm{HCl}$ than in the case of HF dissociation. On Ag(111) the dissociation seems to be exothermic, as 
the products are more stable on surface than the reactants, thus the adsorbed molecule. The dissociation of NO on the noble metals (111)-surfaces shows a late TS like the dissociation of the halides. Therefore, the reactions can be promoted by vibrational excitation of the reactant and the products will be translationally excited. For highly-vibrationally $\mathrm{NO}(v=13,15)$ scattered $\mathrm{Cu}(111)$ this was experimentally observed by Hou et al. [13, 154]. The barrier height is about $50 \%$ lower on $\mathrm{Cu}(111)$ than on the more noble metals $\mathrm{Ag}(111)$ and $\mathrm{Au}(111)$. Thus, highly vibrationally excited NO scattering, e.g. in $v=13$ from $\mathrm{Cu}(111)$ may show dissociation, whereas this seems unlikely for the scattering from the corresponding gold and silver surfaces or at least NO must be in a much higher vibrational state. Moreover, AIMD simulations for these systems should be done to see if dissociation can be observed there when scattering NO in a high $v$-state from these surfaces. For this purpose, a small number of trajectories of $\mathrm{NO}(\nu=13)$ scattering from $\mathrm{Cu}(111)$ at $300 \mathrm{~K}$ with FHI-aims was carried out. The initial translational energy was $0.7 \mathrm{eV}$ and initial vibrational energy was about $2.9 \mathrm{seV}$ (much higher than the barrier height of dissociation $(1.76 \mathrm{eV})$. The outcome of the trajectories showed that about $0.25 \%$ (10 trajectories) of the impinging molecules have a bond length $>2.5 \AA$ when the trajectory was stopped. Since this bond length is much larger than the equilibrium bond length of the molecule and than the distance at the TS we can assume that the molecule is dissociated. The number of $25 \%$ is much smaller than the $87 \%$ observed by Hou et al. which may be due to an important role of non-adiabatic effects in the reaction. However, a larger number of trajectories is necessary to investigate this further. For the oxidation of $\mathrm{CO}$ on $\operatorname{Pt}(111)$ the situation is vice versa. Here the reaction can be classified as an early barrier one because the TS looks more like the reactants. Thus, the reaction product $\mathrm{CO}_{2}$ should be vibrationally excited, and the reaction is promoted by translationally excited reactants. The barrier height of the reaction is influenced by the $\mathrm{CO}$ and $\mathrm{O}$ coverage.

Further AIMD simulations done from the obtained TS in this cell give a mean final translational energy which is in agreement with the one observed in the experiment for the hyper-thermal channel. This is because the AIMD simulations do not include ehps excitation which can occur in the experiment. Moreover, both mean final translational energy values agree well with the energy release as found in the MEP calculations, as most of the released energy is transferred in the vibrational dofs of $\mathrm{CO}_{2}$ as the reaction inherits an early TS. So the MEP calculations together with the AIMD simulations and the experimental observations let us assign this reaction channel to the reaction occurring dominantly on the flat (111)-surface, as published [38]. 


\section{Interaction of diatomic molecules with (111) metal surfaces}

\subsection{Introduction}

One possible way to get a basic understanding of the interaction between a (diatomic) molecule and a (metal) surface is to map the ground state PES of the system of interest. For this purpose we calculate the energy of the system for different nuclear configurations as described in the sections 2.1 and 2.2. This is carried out systematically by sampling the configurational space and producing so-called energy grids. With this we can see where the heights and depths of the energetic landscape are. The former regions show where the interaction between molecule and surfaces is repulsive, whereas deep regions show attractive interaction, and thus where the molecular adsorption on the surface takes place and how strong it is. Furthermore, it is important to know at which distance the repulsion between the molecule and the surface begins. With this information we can say how close an impinging molecule can get to the surface. From this and other considerations it was possible to develop a model to describe the experimentally observed vibrational relaxation behavior of $\mathrm{CO}\left(v_{\mathrm{in}}=17\right)$ from $\mathrm{Au}(111)$, via temporal formation of $\mathrm{CO}^{-}$, when the molecule is close to the surface. This was published in [42]. The data of the energy grids can be used as input to fit them to obtain a PES in a later perspective and can be used to define a starting point for AIMD simulations of scattering experiments of these systems. I did calculations for the following systems: $\mathrm{CO} / \mathrm{Au}(111)$, $\mathrm{CO}$ at Ag-covered $\mathrm{Au}(111)$ and $\mathrm{NO} / \mathrm{Au}(111)$.

\subsection{CO/Au(111)}

To investigate the interaction of $\mathrm{CO}$ and an $\mathrm{Au}(111)$ surface, a $\mathrm{p}(3 \times 3)$ cell with 4 layers was used to model it. The Au atoms were kept fixed at their equilibrium positions, which are determined through the optimized lattice constant $a$. $a$ was found by the procedure described in subsection 4.1.2.1. For the chosen GGA-functionals RPBE and PW91 the following values for $a$ were obtained $4.200 \AA$ and $4.165 \AA$, respectively. To 
avoid interactions between the periodic images of the slab and the diatomic a vacuum distance of $20 \AA$ in $z$-direction was used. The equilibrium bond distance of the CO molecule was defined from its experimental value of $1.12 \AA$. The CO molecule was then placed above the top, bri, fcc , and hcp sites, as defined in Fig. 8a. Here the COM-distance of the molecule to the slab was varied from 1.75 to $8.00 \AA$, with steps of $0.25 \AA$. At each position $\mathrm{CO}$ was oriented with the $\mathrm{C}$-atom down, parallel to the surface along $x$-axis, and with the O-atom down to the slab. So in total the electronic ground state energy $E_{\mathrm{e}, 0}(\boldsymbol{R})$ of 312 nuclear configurations was calculated using VASP. For these calculations the following input parameters were used: exchange and correlation of the electrons were described by RPBE and PW91. Furthermore, the influence of long-range interaction was investigated by using vdW-corrections within the TCHATCHENKO-SCHEFFLER method [152]. The electronic occupation of the valence bands was smeared with a first-order MP function using a $\sigma$ of $0.2 \mathrm{eV}$, and the core electrons were described by the PAW-method. The plane waves were characterized by a cut-off energy of $400 \mathrm{eV}$, and the IBZ was sampled with a $8 \times 8 \times 1 \boldsymbol{k}$-point grid with the $\Gamma$-point included. As the molecule as well as the Au slab does not have unpaired electrons or it is delocalized over all slab atoms, the calculations were carried out in the spin-unpolarized approach.

Furthermore, I investigated the $\mathrm{CO} / \mathrm{Au}(111)$ interaction with $\mathrm{FHI}$-aims for the two XC functionals mentioned above as well as with vdW corrections. The system was again described by a $\mathrm{p}(3 \times 3)$ slab and 4 layers built up by the optimized lattice constants of $4.200 \AA$ for RPBE and $4.165 \AA$ for PW91, and a vacuum width of $20 \AA$. The CO molecule was again defined from its equilibrium value of $1.12 \AA$ and placed above the 4 adsorption sites at the same COM distances to slab as in case of the VASP calculations. The following parameters were used for the calculations: the reciprocal space was sampled with a $8 \times 8 \times 1 \boldsymbol{k}$-point grid including the $\Gamma$-point, the electronic occupation was modeled by a Gaussian function with a width of $0.2 \mathrm{eV}$, the basis sets of $\mathrm{C}, \mathrm{O}$ and Au were defined by tight-settings. The calculations were carried out in the spin-unpolarized approach as for the ones done with VASP.

On the left side of the upper panel of Fig. 34 one sees that the interaction energy at top site does not have a stable minimum when CO approaches the Au slab. However, in the "C-down" orientation a local energy minimum occurs when the $\mathrm{C}$ atom is about $2.1 \AA$ above a surface atom. For the two other molecular orientations (parallel and "O-down"), the interaction energy just increases when the molecule comes closer to the slab. This increase has a higher slope when the $\mathrm{O}$-atom is turned to the metal atom. As the interaction between carbon and gold is less repulsive than for $\mathrm{O}$ atom, it seems to be more likely that the "C-down" orientation also shows energetic minima at other sites on the surface. From the upper-right panel of Fig. 34 we can state that the interaction 
between the C-atom, which directly sits above a gold atom, is less repulsive than in the case that $\mathrm{CO}$ sits between two top layer atoms, thus approaches above the bri site. When CO directly approaches above the $f c c$ or $h c p$ site, the PES also shows a local minimum, but it is less stable than the minimum at the top site. This latter minimum occurs when the COM of the molecule is about $2.75 \AA$ away from the surface, whereas the minima of the three other sites occur closer to the surface. Furthermore, before the molecule gets into the minima it has to overcome a barrier which is just $0.1 \mathrm{eV}$ for the top site but around $0.4 \mathrm{eV}$ for the other three sites. The lowest of these three barriers appears at the $b r i$, and the highest at $f c c$ site. Therefore, the molecule-slab repulsion is strongest at the fcc and weakest at the top site.
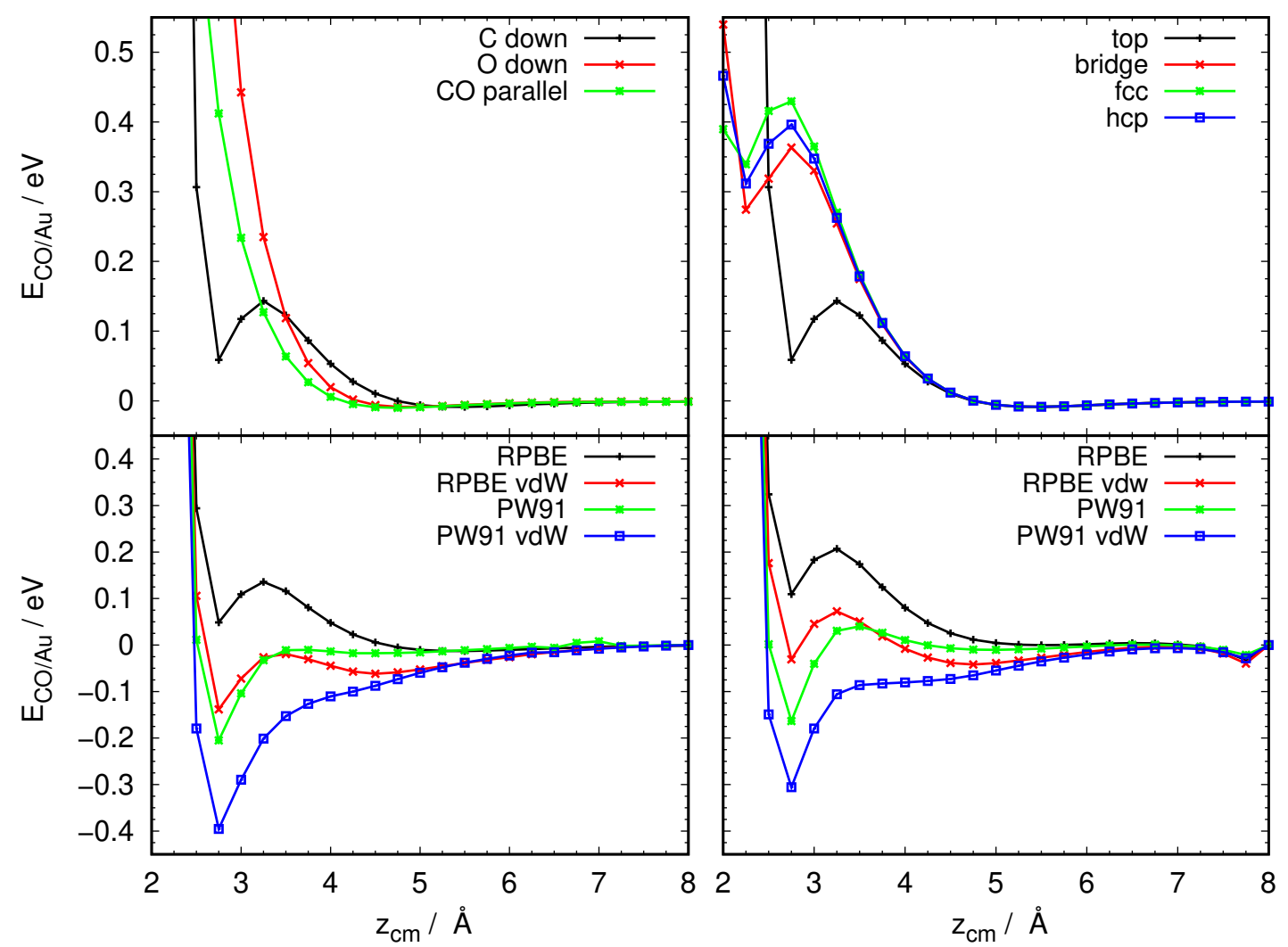

Fig. 34: $\mathrm{CO} / \mathrm{Au}(111)$ interaction energy as function of the CO COM-distance to the slab $\left(z_{\mathrm{cm}}\right)$ for various conditions: different CO orientations at the top site with RPBE (top left), "C-down" above different sites with RPBE (top right), "C-down" at the top site for different XC-functionals (leftbottom), the same as left-bottom but using FHI-aims (right bottom) instead of VASP.

The interaction between $\mathrm{CO}$ and $\mathrm{Au}(111)$ is not attractive when calculated with RPBE, this seems to be due to the XC functional or due to long-range vdW interactions. The choice of another functional like PW91 or the use of vdW corrections for the system shift the local minimum for the "C-down" orientation at the top site to a global minimum on the PES as shown in the left-bottom panel of Fig. 34. Thus, the use of vdW corrections 
leads to a stronger attraction which also begins when $\mathrm{CO}$ is at a larger distance from the slab. For PW91 the attraction between $\mathrm{CO}$ and $\mathrm{Au}$ is stronger than for RPBE, and for the former functional no energetic barrier occurs.

Results for the same nuclear configurations and the different functionals with FHI-aims are shown in the bottom-right panel of Fig. 34. The overall trend in the interaction energy for the functionals is similar to the one found with VASP, but the moleculeslab interaction is more repulsive with the all-electron code. Thus, the energy profiles calculated with FHI-aims reveal well depths which are about $0.1 \mathrm{eV}$ higher than those calculated with VASP. Furthermore, the calculations with FHI-aims show a higher barrier when the molecule approaches the surface than the ones done with VASP. A feature which occurs in all FHI-aims calculations is a dip in the interaction energy of $0.04 \mathrm{eV}$ at a COM distance of the molecule to the slab of $7.75 \AA$ and is not seen for VASP. This as well as the other differences seems to be attributed the differences in the codes, and the different approaches of wave functions in the codes may be responsible for this. But also differences in the input files may cause these differences. However, in principle the codes show a similar behavior in the interaction energy.

Finally, RPBE with vdW-corrections and PW91 show an adsorption minimum close to the experimental value adsorption energy of $\mathrm{CO}$ on an $\mathrm{Au}(111)$ surface which is between 0.15 and $0.18 \mathrm{eV}$ [155]. More recently, the chemisorption and physisorption processes for $\mathrm{CO}$ at $\mathrm{Au}(111)$ was experimentally investigated [156] and the desorption barrier for the physisorbed states was determined to $0.120 \mathrm{eV}$ and the barrier for the chemisorbed state was determined to $0.08 \mathrm{eV}$.

When considering an initial COM-distance of CO to slab for AIMD simulations a value above $5 \AA$ seems to be a reasonable choice as the interaction energy $E_{\mathrm{CO} / \mathrm{Au}}$ between both parts of the total system is constant, at least without vdW-corrections. For the calculations with the latter corrections a more distant starting point is reasonable. However, a larger molecule surface distance leads to longer trajectories when investigating scattering events at the surface and therefore the computational effort increases. The simulations carried out with vdW-corrections can be started from a COM distance of the molecule to the surface of $z_{\mathrm{cm}}=5.5 \AA$, thus the same starting point as for the simulations without the correction, to reduce the computational effort.

\subsection{CO/Ag(111) and CO at Ag-covered Au(111)}

I also performed calculations for $\mathrm{CO}$ at $\mathrm{Ag}(111)$ as well as at $\mathrm{Ag}$-covered $\mathrm{Au}(111)$. These are done to see if there is a strong difference between the interaction of CO with the different surfaces compared to the pure cases of silver and gold. Since strong differences 
in the molecule-surface interaction like adsorption minima could be a reason why the translational energy distributions of $\mathrm{CO}(\nu=2)$ scattered from those surfaces show significant differences. As for $\mathrm{Au}(111)$ the slab atoms were held at their equilibrium positions which are determined by a lattice constant $a=4.20 \AA$ for RPBE, so the same as for $\mathrm{Au}(111)$. So there were no structural problems when I built the Ag-covered surfaces as described in chapter 5 . Thus, the layers with Au atoms were consecutively exchanged with layers of $\mathrm{Ag}$ atoms. Moreover, a $\mathrm{p}(3 \times 3)$ slab with 4 layers and again a vacuum distances of $20 \AA$ in the $z$-direction was used for every case. The calculations in this section were carried out with VASP with input parameters as described in the previous section (7.2).

Since, on the PES for $\mathrm{CO} / \mathrm{Au}(111)$ only a (local) minimum occurs close to the slab when $\mathrm{C}$ is turned to it, I especially calculated the energies for those configurations. Further I focused on the top and $f c c$ sites, where the attraction of $\mathrm{CO}$ on $\mathrm{Au}(111)$ was strongest and weakest, respectively, see previous section.
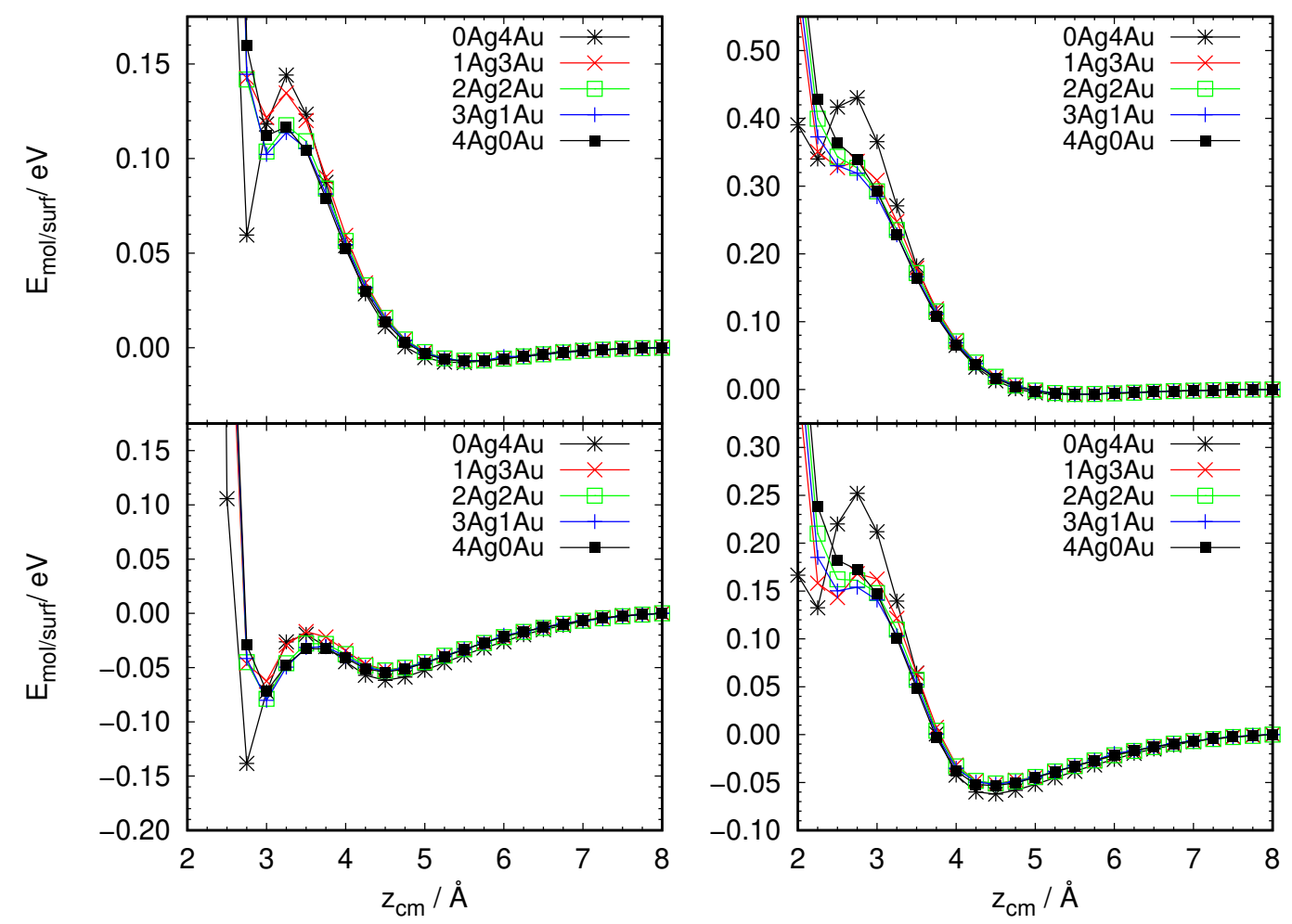

Fig. 35: Interaction energy ( $\left.E_{\mathrm{mol} / \mathrm{surf}}\right)$ of $\mathrm{CO}$ and different metal(111) surfaces, when $\mathrm{C}$ is turned to the slab. On the left $\mathrm{CO}$ is set above top and on the right $\mathrm{CO}$ is set at the $f c c$ site. The upper panels show the calculations with RPBE and the bottom ones shows them including the vdW-corrections.

In Fig. 35 the interaction energy for $\mathrm{CO}$ at the different model surfaces is shown when the molecule approaches the corresponding slab. Again the use of vdW-corrections 
leads to a stronger attraction between molecule and slab than in the case without them. The physisorption as well as the chemisorption is more attractive in the case with vdW-corrections. Further the preference in adsorption site is the same thus top is more attractive than the $f c c$ site. For the different slabs, the gold one displays stronger attraction to $\mathrm{CO}$ than the pure silver slab and the ones which consists of both metals. Even with vdW-corrections and with the molecule at top site (left-bottom of Fig. 35) the energy profiles show only a flat well depth when the COM of CO is around $2.75 \AA$ away from the corresponding surface. However, the calculations with slabs containing silver atoms all deliver very similar energy profiles and just show small differences in their adsorption minima close to the surface. Thus, any differences in the translational energy distributions of CO scattered from these different surfaces cannot be explained with differences in PESs of these systems.

\subsection{NO at $\operatorname{Au}(111)$}

Since experimental results for scattering of highly-vibrationally excited NO from $\mathrm{Au}(111)$ suggest that the vibrational energy transfer to the surface is strongly governed by nonadiabatic effects, it is necessary to include theories to describe these experiments. One theoretical approach is IESH which was successfully used to describe some experimental features [46]. It needs more than one PES as a building block. This means one PES for the neutral $\mathrm{NO}$ and one PES for the ionic $\mathrm{NO}^{-}$. One approach to obtain those PESs is to calculate the electronic ground and excited states for NO at Au(111) using DFT, and then apply a diabatization procedure [157].

First, I calculated the energy for an NO molecule at an Au(111) surface for different molecular orientations and adsorption sites. For these calculations I used the spinpolarized approach of the GGA with RPBE as employed in VASP. The total magnetic moment of the system was allowed to relax as well as kept fixed to $1($ NUPDOWN=1) to see if the calculations converge to a different ground state. The latter treatment of the spin state was also used by RoY et al. [157]. An MP1 smearing with a width of $0.2 \mathrm{eV}$ for the electronic occupation and $\boldsymbol{k}$-point grid of $4 \times 4 \times 1$ including the $\Gamma$-point to sample the reciprocal space were used. Plane waves up to an energy of $400 \mathrm{eV}$ were included in the wave functions. The $\mathrm{Au}(111)$ surface was modeled by a $\mathrm{p}(3 \times 3)$ slab with 4 layers, built up by an optimized lattice constant of $4.20 \AA$, and a vacuum width of $20 \AA$ in $z$-direction was set. The NO molecule, described by its experimental equilibrium bond distance of $1.15 \AA$, was set with its COM between 2 and $8 \AA$ away from the slab.

In the upper left panel of Fig. 36 the calculated interaction energy for $\mathrm{N}$-down for different adsorption sites is shown. Far away from the surface some points in the 
configurational space leads to about $0.3 \mathrm{eV}$ higher energy than other points, which have a similar configuration. These jumps are artifacts due to bad converged results, i.e. convergence to a wrong electronic ground state. The reason for these artifacts in the energy profiles seems to be differences in the spin state. This can be concluded from the bottom left panel of Fig. 36, where the final total magnetic moment of the system for the corresponding calculations is shown. Thus, if the final magnetic moment differs from 1 or -1 the energy profile exhibits a jump. Interestingly, the calculations with the fixed total magnetic moment for the $f c c$ site (green stars) show always a higher energy than the calculations where the total magnetic moment is relaxed (green crosses). Also, it seems to be problematic to say something about the interaction in the case of VASP, due to the jumps in the energy profiles. However, in this case no attractive interaction for any adsorption site can be seen.
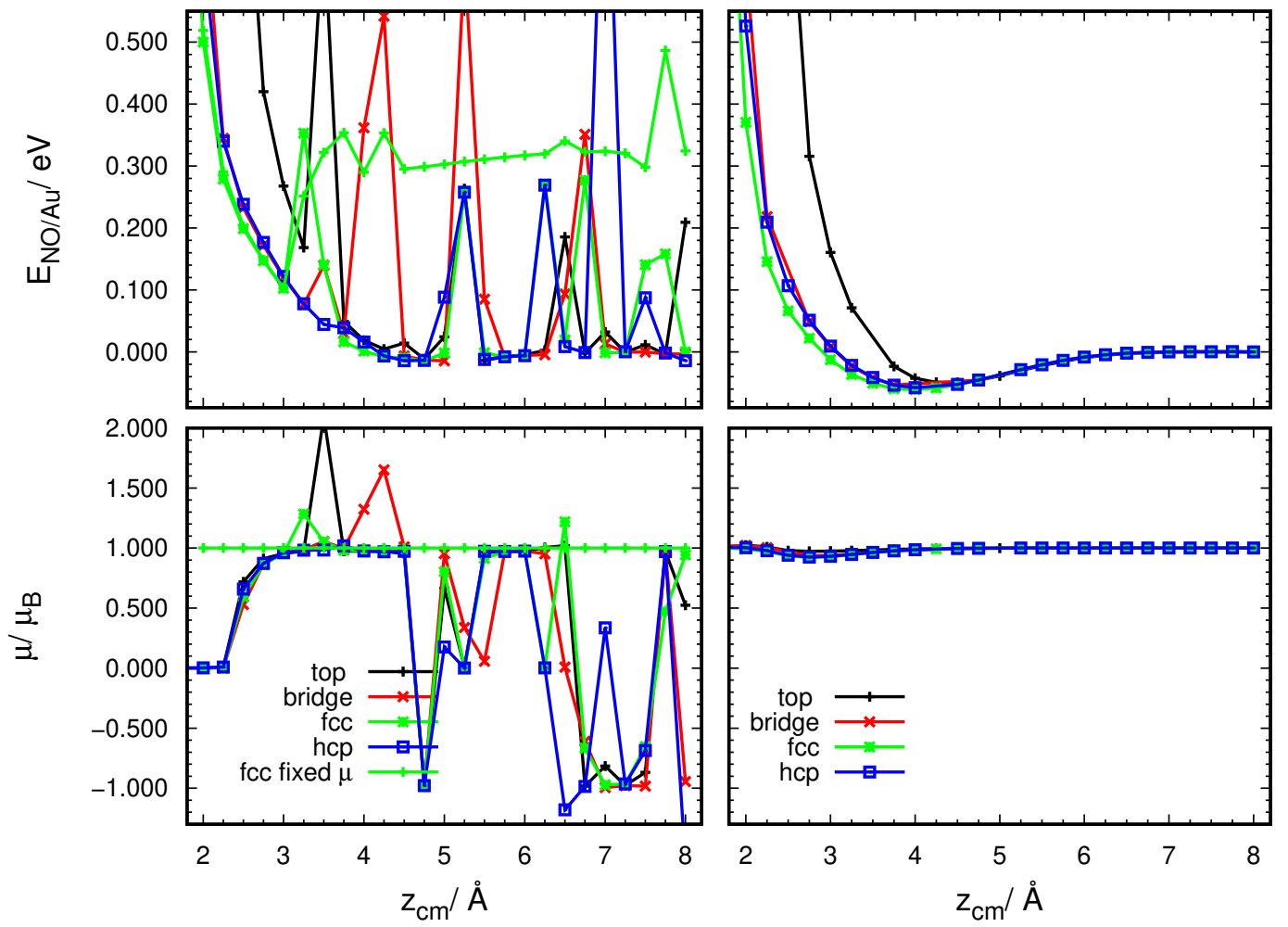

Fig. 36: NO/Au(111) interaction energy as function of the NO COM distance to the surface for Ndown and different adsorption sites for VASP (left) and FHI-aims (right) calculated with RPBE. The bottom panel shows the corresponding final magnetic moment $\mu$ of the calculations for VASP (left) and FHI-aims (right). The green stars and the green crosses on the left side represent calculations along the $f c c$ site with relaxed and fixed total magnetic moment, respectively.

For this reason, I did calculations with the all-electron code FHI-aims. This DFT code provides a better means to control the spin state during the calculation, due to implementation of localized basis sets. The calculations were done with the following parameters: RPBE functional in the spin-polarized approach with fixed moment of 1 
and an initial moment for $\mathrm{N}$ and $\mathrm{O}$ of 3 and 2, respectively. The electronic occupation was described by a Gaussian function with a width of $\sigma=0.2 \mathrm{eV}$ and the IBZ was sampled by a $4 \times 4 \times 1 \boldsymbol{k}$-point grid. The basis sets were defined by the light settings. The simulation cell consists of a $\mathrm{p}(3 \times 3)$ slab with 4 layers, with atoms at their equilibrium positions given by $a=4.20 \AA$ and a width of the vacuum layer of $20 \AA$. The NO bond length was set to its experimental value of $1.15 \AA$.

The outcome of these calculations are shown in the top right panel (interaction energy) and bottom right panel (final total magnetic moment) of Fig. 36. The energy profiles show no jumps and the final magnetic moment differs only slightly from 1 , when the molecule comes close to the surface. Also, a small well depth of $0.06 \mathrm{eV}$ is observed, when the COM of NO is about $4 \AA$ away from the surface. The molecule surface attraction is strongest when the molecule is above the $f c c$ site. When NO approaches from above top site the repulsion between $\mathrm{NO}$ and $\mathrm{Au}(111)$ starts around $1 \AA\left(z_{\mathrm{cm}}=4 \AA\right)$ further away than above the three other sites. The energy profiles calculated with FHI-aims were used to estimate the molecule-surface distance at which NO has contact to the repulsive part of the interaction potential, as reported in [42]. This position was taken as the COM of the molecule to the surface at which the potential energy has a value of $0.5 \mathrm{eV}$ (this corresponds to the experimental incidence translational of the impinging molecule which is $\approx 2 \AA$ ). With this it was possible to determine the image charge stabilization of the anionic molecule, and from this the vertical electron binding energy (VEBE) of the closest approach from NO to the surface was calculated.

With this it was possible to develop a model to explain the differences in the vibrational relaxation behavior of highly vibrationally scattered NO and CO from Au(111), via transient formation of an anion at the surface with ehp excitation. Because the VEBE is higher for $\mathrm{NO}(v=16)$ than for $\mathrm{CO}(v=17)$ it is more likely to form anions at surface in the first case, and therefore vibrational relaxation should be stronger for this molecule. This model could also explain the difference in the vibrational relaxation of NO and CO in different initial vibrational states scattered from different metal surfaces and was proposed in [42] and further applied to other systems in [43].

Finally we can say that FHI-aims perform better with respect to convergence than VASP, at least for the chosen input setting. But unfortunately, it is not possible to do calculations with an external electric field and pbc in FHI-aims [105]. Since such calculations are necessary to account for the excited state of the NO/Au(111) system it is mandatory to have two potential energy surfaces to do IESH simulations - I went back to VASP code. 


\subsubsection{Convergence tests for NO/Au(111)}

Before I did the extensive calculations for $\mathrm{NO} / \mathrm{Au}(111)$, to obtain the input data for the diabatic potential, I made convergence tests for some important input parameters such as the cut-off energy $E_{\text {cut }}$ of the plane waves, the size of the $\boldsymbol{k}$-point grid, and the smearing function and its width $\sigma$. The tests were done for the molecule above the $f c c$ site close to the surface $\left(z_{\mathrm{cm}}=2.0 \AA\right)$ as well as for the molecule far away from the surface $\left(z_{\mathrm{cm}}=8.0 \AA\right)$ for three different molecular orientations: $\mathrm{N}$ or O oriented towards the surface ("N-down" or "O-down") and NO parallel aligned with respect to the surface normal. The tests were done with the RPBE functional within the spinpolarized approach with full relaxation of the total magnetic moment. But the initial magnetic moment (MAGMOM) was set to 3 for $\mathrm{N}$ and -2 for $\mathrm{O}$.

Since $E_{\text {cut }}$ describes the quality of the wave function the choice of $E_{\text {cut }}$ is most important. For these calculations a $4 \times 4 \times 1 \boldsymbol{k}$-point grid and MP1 smearing with a width of $0.2 \mathrm{eV}$ were used.
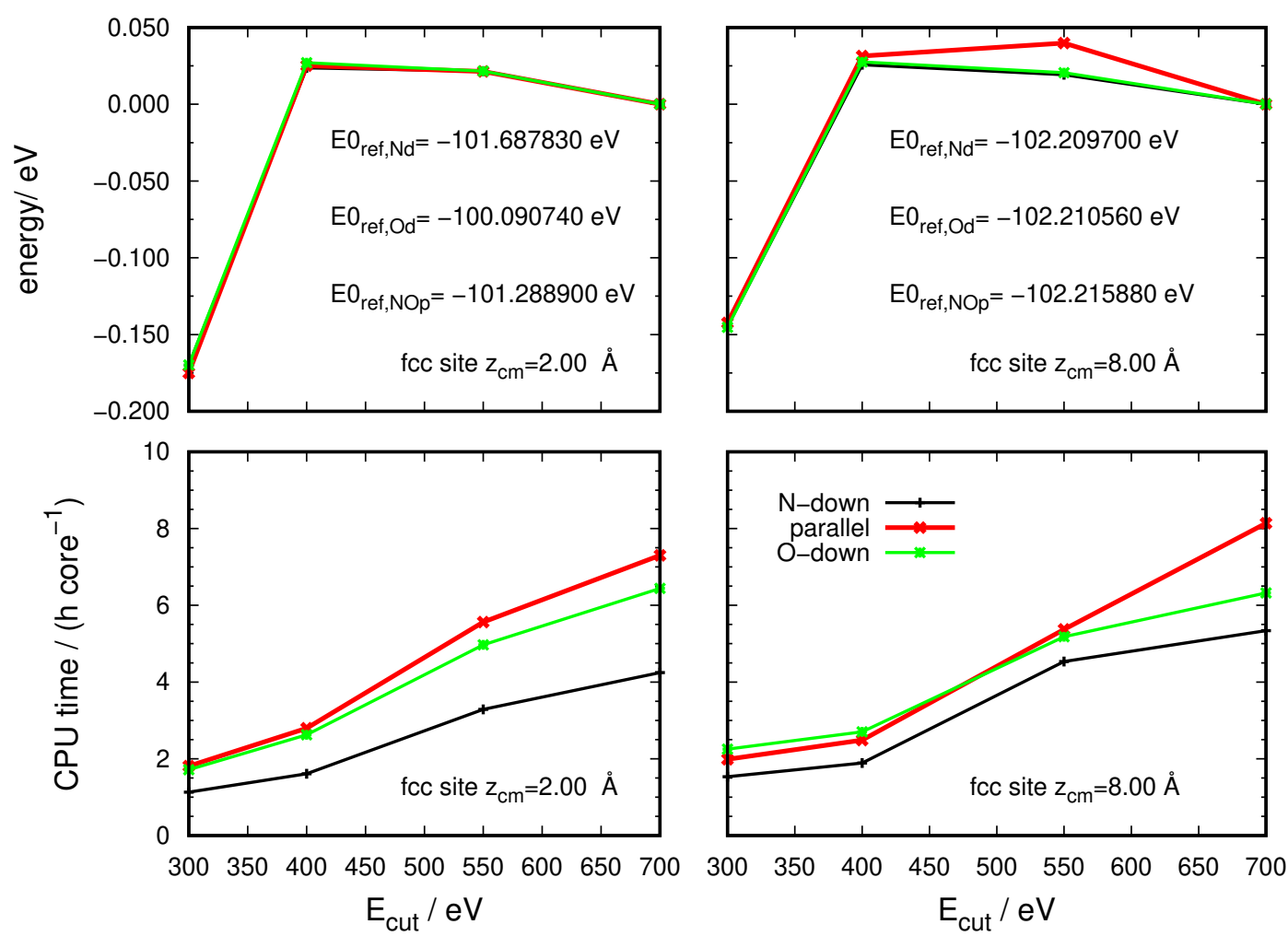

Fig. 37: Energy (upper panel) and computational time (lower panel) vs. cut-off energy ( $\left.E_{\text {cut }}\right)$ for NO at $z_{\mathrm{cm}}=2.0 \AA$ (left) and $z_{\mathrm{cm}}=8.0 \AA$ (right) above the $f c c$ site of an Au(111) for different orientations. The black, red and green lines represent $\mathrm{N}$-down, parallel and $\mathrm{O}$-down cases, respectively. The numbers give the used reference energy. 
Fig. 37 shows the energy (upper panel) and the computational time (bottom panel) as function of the cut-off energy ( $\left.E_{\text {cut }}\right)$ for different molecule-surface distances and molecular orientations. For the smallest $E_{\text {cut }}$ of $300 \mathrm{eV}$ the energy is not converged, whereas the cut-off of $400 \mathrm{eV}$ is only $0.030 \mathrm{eV}$ away from the value at $700 \mathrm{eV}$. Within this accuracy the results can be seen as converged.

Hence, using a cut-off energy of $400 \mathrm{eV}$ gives converged results independent of the molecular distance to the surface. The computational time endorses the choice of $E_{\text {cut }}=400 \mathrm{eV}$, because these calculations are more than three times faster than those ones with the higher cut-off energy of $700 \mathrm{sieV}$. This is observed for both investigated molecule-surface distances and the three molecular orientations.
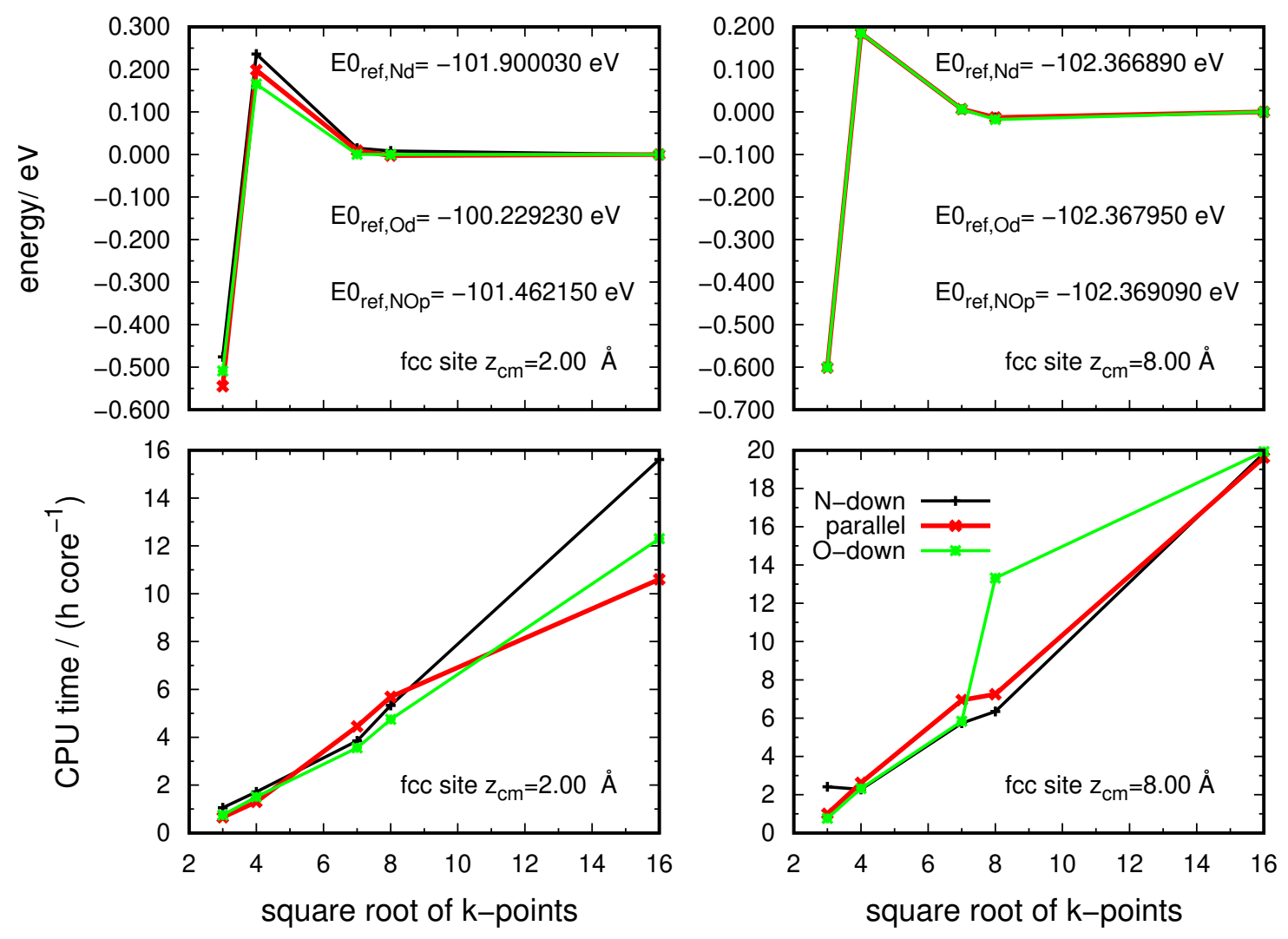

Fig. 38: energy (upper panel) and computational time (lower panel) vs. square root of $k$-points for NO at $z_{\mathbf{c m}}=2.0 \AA$ (left) and $z_{\mathbf{c m}}=8.0 \AA$ (right) above the $f c c$ site of an $A u(111)$ for different orientations. The black, red and green lines represent $\mathrm{N}$-down, parallel and $\mathrm{O}$-down cases, respectively. The numbers give the reference energy, respectively.

The test of the $\boldsymbol{k}$-point grid size, done with $E_{\text {cut }}=400 \mathrm{eV}$ and a MP1 smearing with $\sigma=0.2 \mathrm{eV}$, shows that convergence with respect to energy within $20 \mathrm{meV}$ is reached when the IBZ is sampled with $49 \boldsymbol{k}$-points, either close or far away to the surface and also for the tested orientations (see Fig. 38). Unfortunately, increasing the $\boldsymbol{k}$-point grid size 
from $4 \times 4 \times 1$ to $7 \times 7 \times 1$ more than doubles the computational time for the calculations (see bottom panel of Fig. 38).

It is known in DFT calculations that the energy difference is given more accurately than the absolute values. A comparison of these energy differences for different sizes of the $\boldsymbol{k}$-point grids is shown in Fig. 39.

The upper panel shows calculations of the molecule-surface interaction energy for different distances for a $4 \times 4 \times 1$ and the middle panel shows the same for a $8 \times 8 \times 1 \boldsymbol{k}$ point grid. The reference energy for the different grids (given in every graph) differs by about $0.2 \mathrm{eV}$ for both adsorption sites.

The lower panel of Fig. 39 shows that the differences in the referenced energy $E_{\mathrm{mol} / \mathrm{surf}}$ between an $8 \times 8 \times 1$ and a $4 \times 4 \times 1 \boldsymbol{k}$-point grid are in the sub-meV-range far away from the surface and under $50 \mathrm{meV}$ closer to the surface. The variation is $50 \mathrm{meV}$ only for the $f c c$ site for the $\mathrm{N}$-down orientation very close to the surface. Because 50 simeV is the upper limit, so to speak, from converged results, I tested this behavior for configurations from trajectories where the COM is closest to the surface. The tests show that the energy differences between both $\boldsymbol{k}$-point grids are all below $50 \mathrm{meV}$ for those configurations. So with respect to energy differences a $4 \times 4 \times 1 \boldsymbol{k}$-point grid shows a similar behavior than a larger $\boldsymbol{k}$-point grid and therefore it is suitable for the desired purposes.

After founding reliable values for the cut-off energy and the $\boldsymbol{k}$-point grid size I come to the convergence test with respect to the smearing function and its width $\sigma$, which were again done at the same distances and molecular orientations as the tests before. I did tests for four different smearing functions namely Fermi [91], Gaussian [92], MP [93] in first and second order (see section 2.3) and varied the width from 0.02 to $0.80 \mathrm{eV}$.

Since the smearing function describes the electronic occupations of bands, it is a critical parameter to reach to the correct electronic ground and spin states.

Fig. 40 shows that the entropic contribution to the energy $T S$, which is the difference between free energy (F) and energy without entropy (EwoS), increases with $\sigma$, for all tested smearing functions. Especially for the Gaussian and Fermi smearing this contribution goes up to $5 \mathrm{eV}$ and $40 \mathrm{eV}$, respectively. On the contrary the more sophisticated MP functions show an entropic energy contribution relatively small, just up to $0.5 \mathrm{eV}$. When $\sigma$ is smaller than $0.1 \mathrm{eV}$ all functions lead to the energies which differ about $0.01 \mathrm{eV}$.

But choosing $\sigma$-values $<0.10 \mathrm{eV}$ or $>0.4 \mathrm{eV}$ leads to convergence problems when the molecule is distant to the surface $\left(z_{\mathrm{cm}}=8.00 \AA\right)$ or at least to an increasing in computational time. Convergence problems including either calculations end in the wrong spin state or calculations which do not reach the abortion criterion within the maximum number of scf-cycles. 
top
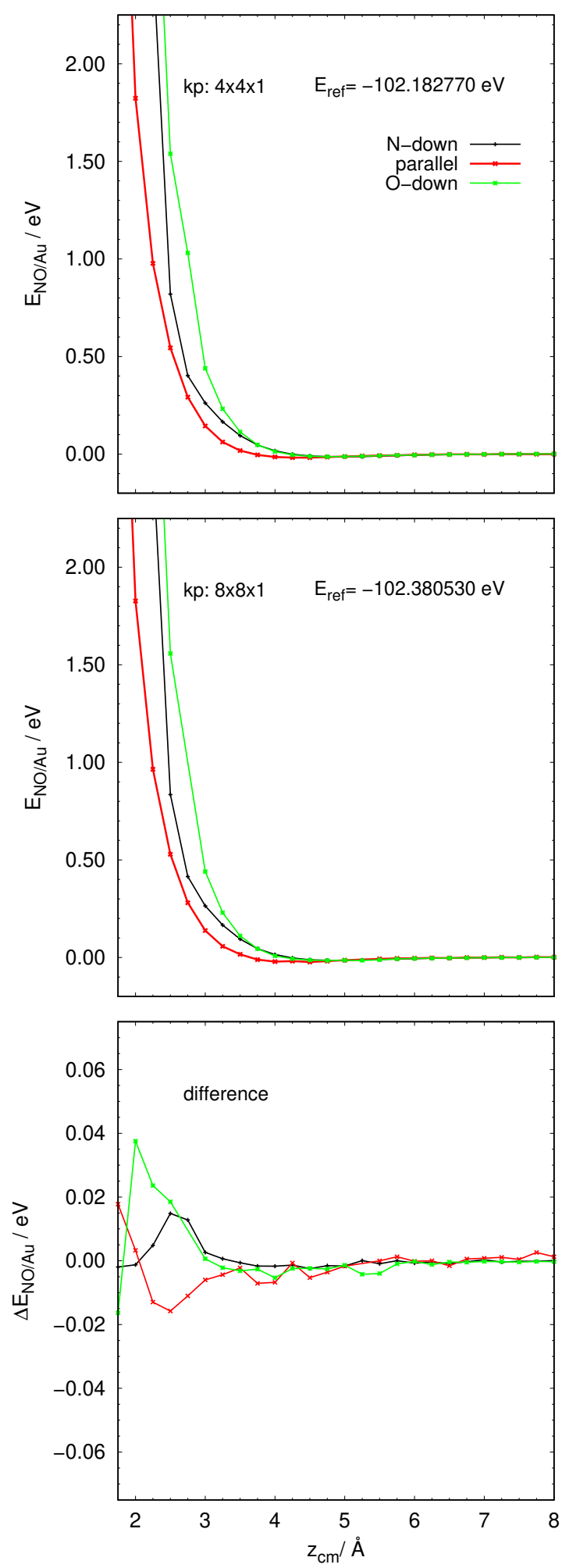

fcc
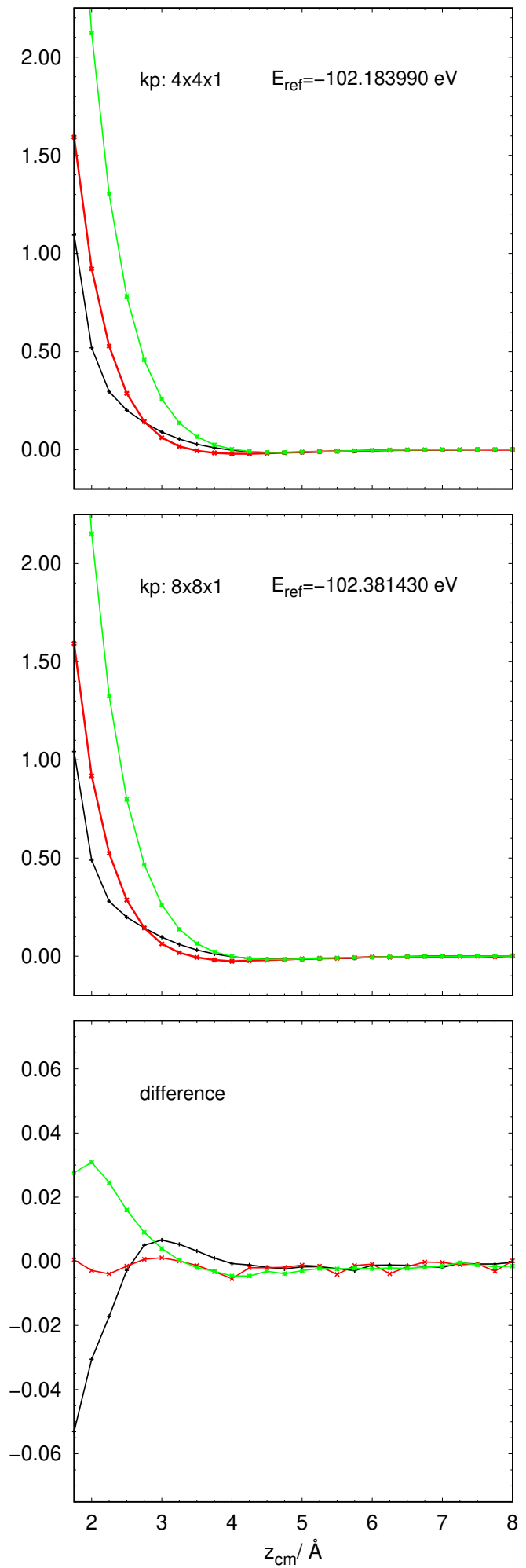

Fig. 39: Molecule-surface interaction energy $E_{\mathrm{NO} / \mathrm{Au}}$ vs. $z_{\mathrm{cm}}$ for a $4 \times 4 \times 1$ (upper panel), a $8 \times 8 \times 1 \mathrm{k}$ point grid (middle panel) and their differences (bottom panel); for the top (left) and fcc sites (right), for three molecular orientations. 

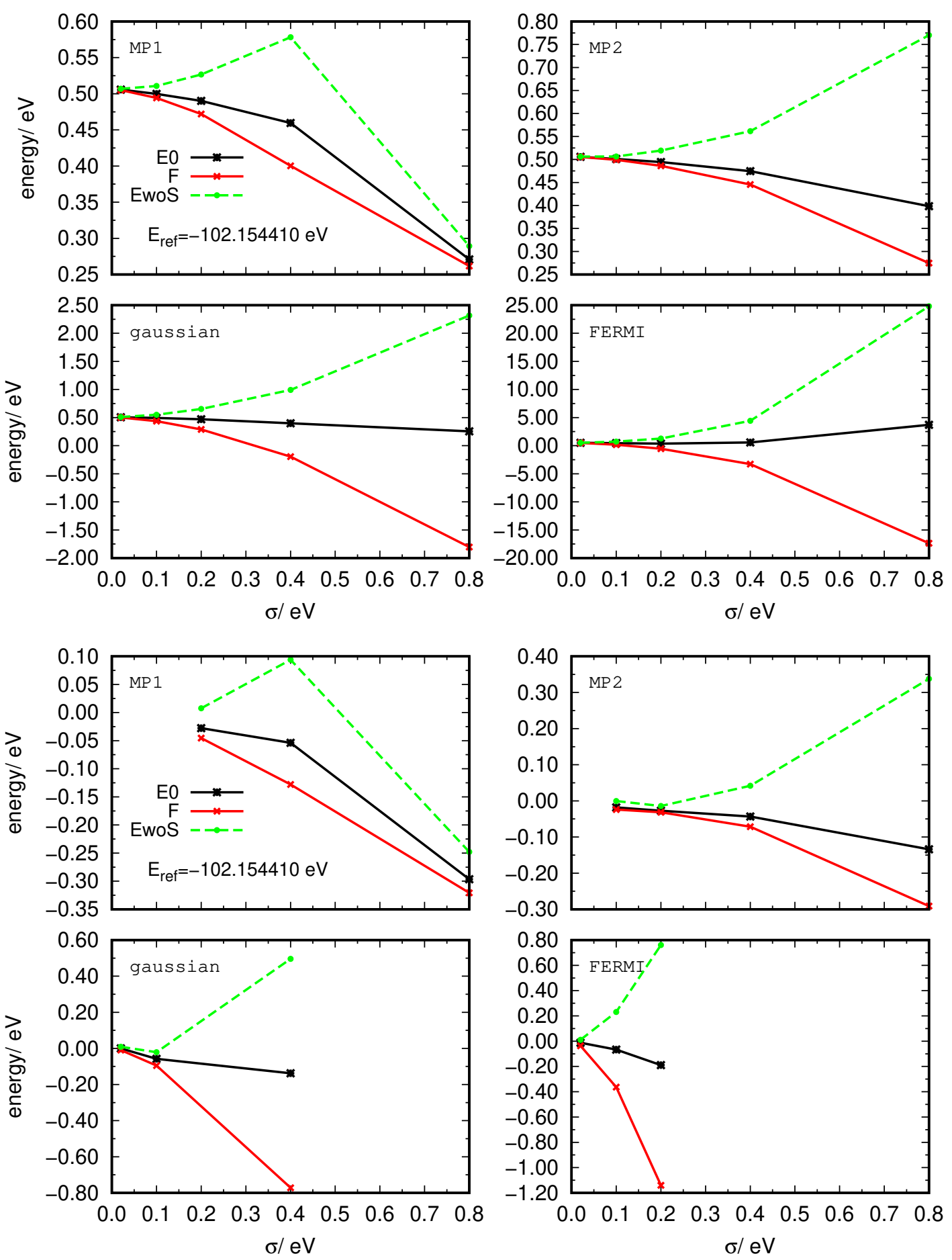

Fig. 40: Free energy ( $F$, red), energy without entropy (EwoS, green) and E0 (black) as a function of $\sigma$ for MP1- (upper left), MP2- (upper right), Gaussian- (lower left) and Fermi-smearing (lower right). The NO molecule is above the $f c c$ site $\left(z_{\mathrm{cm}}=2.00 \AA\right.$ (upper panel) $\& z_{\mathrm{cm}}=8.00 \AA$ (lower panel)) $\mathrm{N}$-down oriented. 

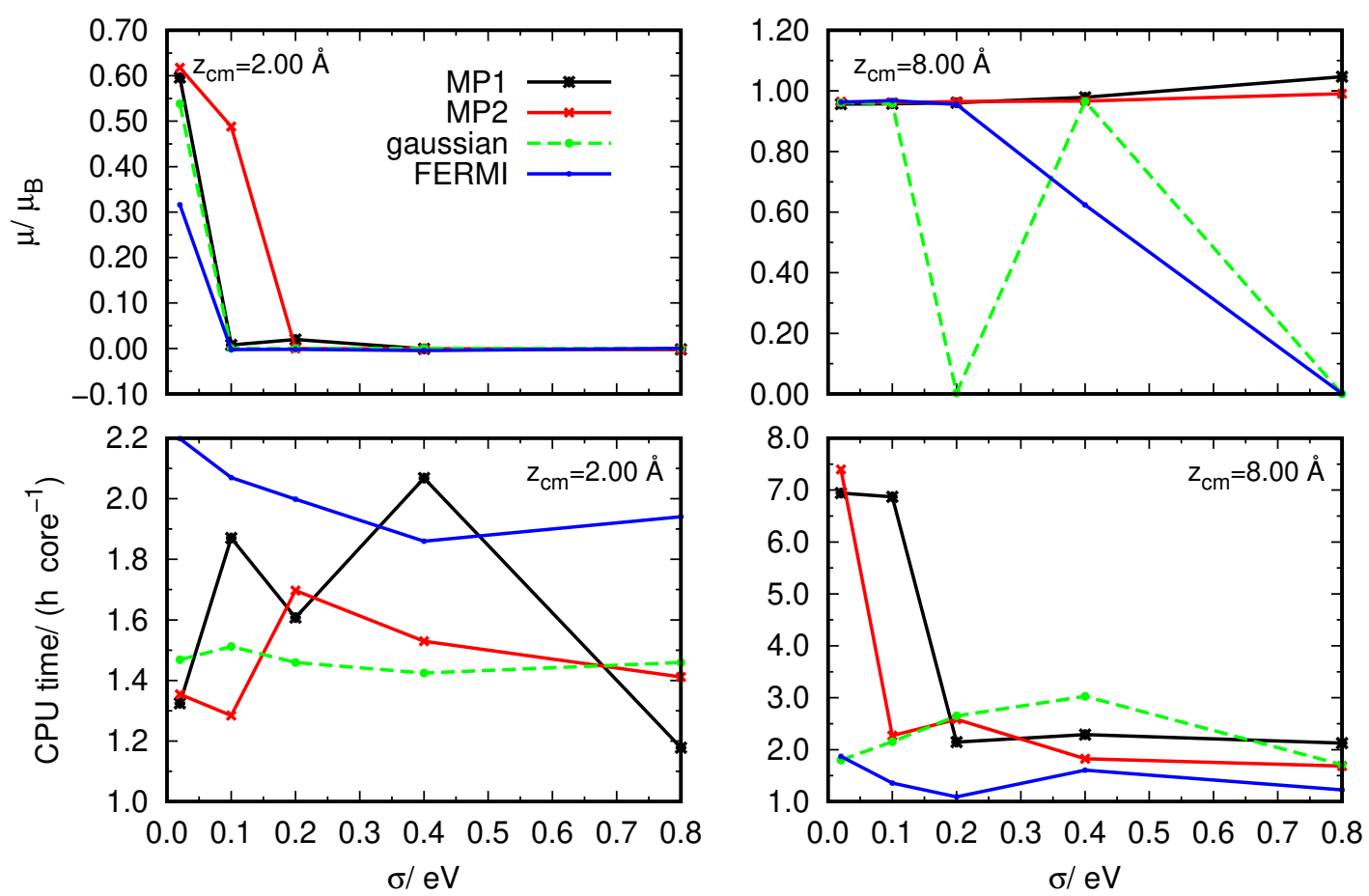

Fig. 41: Magnetic moment $(\mu)$ and computational time vs. $\sigma$ for MP1 (black), MP2 (red), Gaussian (green) and Fermi-smearing (blue), for $\mathrm{N}$-down orientation above $f c c$ site at $\mathrm{z}_{\mathrm{cm}}=2.00 \AA$ (left panel) and $\mathrm{z}_{\mathrm{cm}}=\mathbf{8 . 0 0} \AA$ (right panel). 
If the molecule is close to the surface only a very small width leads to a different spin state, but overall all the methods give the same spin state, thus this is independent of $\sigma$. The calculations need about $20 \%$ more time when Fermi-smearing is used compared to the other smearings, which need around 1.3 to $1.6 \mathrm{~h} \mathrm{core}^{-1}$ of computational time. It is shown in Fig. 41 in the left panel for a "N-down" oriented molecule but also observed for other molecular orientations. When the molecule is set to $z_{\mathrm{cm}}=8.00 \AA$ the calculations show jumps in the magnetic moment for the Gaussian and Fermi function when $\sigma>$ $0.2 \mathrm{eV}$, but neither MP1 nor MP2 show these problems and show a total magnetic moment of $1 \mu_{\mathrm{B}}$, which seems to be correct as the molecule has one unpaired electron. But both functions need an increasing computational effort for smearing widths $<0.2 \mathrm{eV}$. For the two other smearing functions (Gausssian and Fermi) the computational time is nearly independent of $\sigma$. The calculations with the Fermi-smearing are faster than those with MP functions, the latter are a bit faster than those using the Gaussian-smearing (when $\sigma>0.2 \mathrm{eV}$ ). To conclude these examinations of the smearing parameters, the MP functions lead to converged results with the correct spin state for $\sigma 0.1-0.4 \mathrm{eV}$ in a feasible time. The other two smearing functions Fermi and Gaussian need more computational resources and get into wrong spin state. So the choice of MP1 with $\sigma=0.2 \mathrm{eV}$ seems to be an appropriate set of parameters.

So far I just looked at the $\sigma$-dependence of absolute energies for distinct nuclear configurations, but the energy difference is more revelevant between two configurations for a certain smearing width. Thus, a comparison similar to that for the $\boldsymbol{k}$-point grid (see Fig. 39) was done. This energy difference is relatively small and just goes up to $120 \mathrm{meV}$ for $\sigma=0.8 \mathrm{eV}$, as shown in the lower panel of Fig. 42. This figure clearly gives evidence that the sensitivity of the choice of $\sigma$ is not important as long as one is interested in energy differences, because the energetic behavior with varying $\sigma$ is very similar for different configurations.

So from these convergence tests I choose a $4 \times 4 \times 1 \boldsymbol{k}$-point grid to sample the IBZ and a MP1 function with $\sigma=0.2 \mathrm{eV}$ to smear the electronic occupations of the metallic bands for the calculations to produce the DFT data for the PES for NO/Au(111).

\subsubsection{Energy grid for NO/Au(111)}

In the following, I did static calculations to sample the configurational space with the molecule at different distances from the gold surface (atoms at the equilibrium positions): $z_{\mathrm{cm}}=1.75-8.0 \AA$ with a step size of $0.25 \AA$; at different adsorption sites: top, bridge, fcc and hcp, and three molecular orientations: $\mathrm{N}$ headed for the surface 


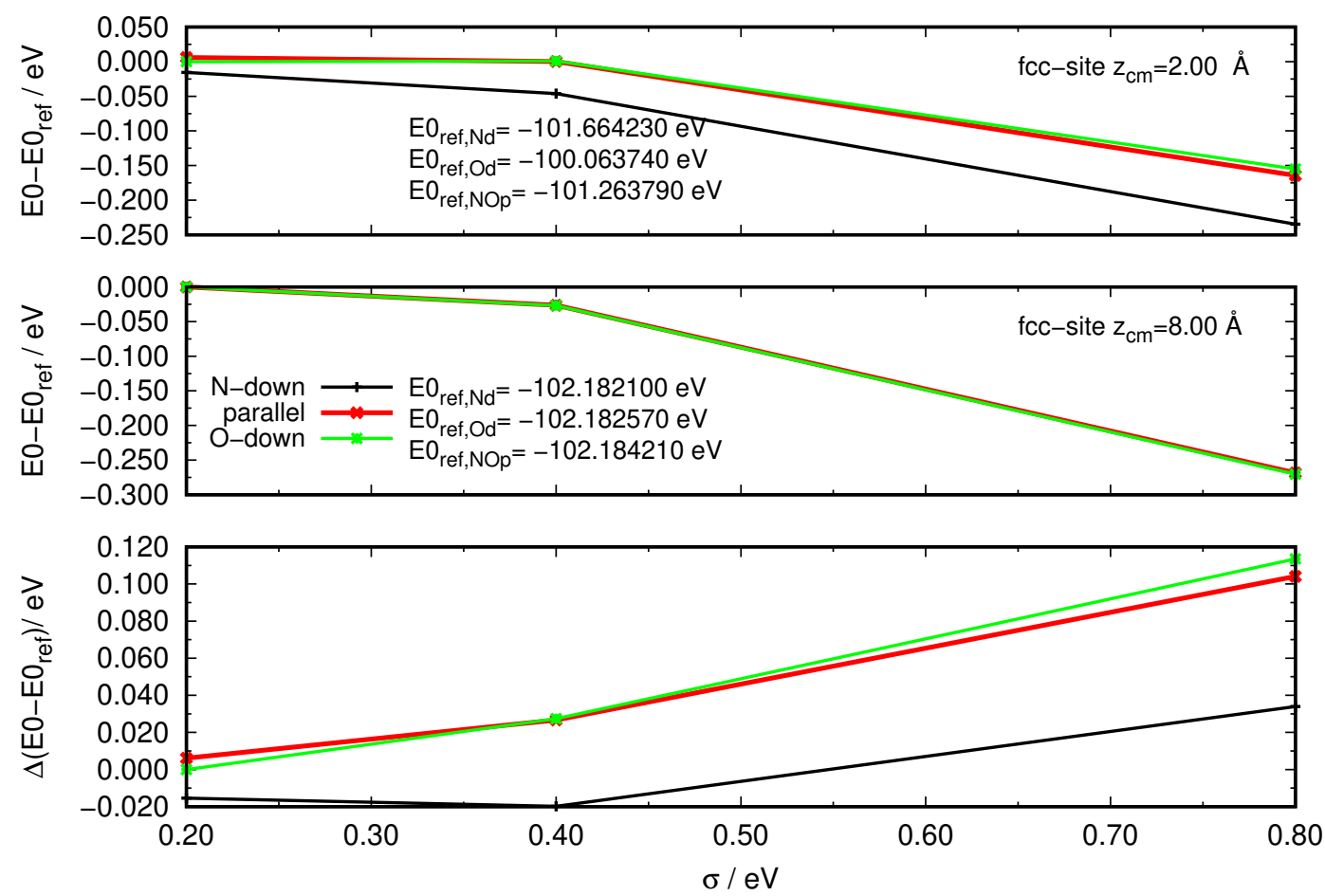

Fig. 42: Energy vs. smearing width $\sigma$ for MP1 smearing function "N-down" (black), parallel (red) and "O-down" (green) orientations above the $f c c$ site for $z_{\mathrm{cm}}=2.00 \AA$ (upper panel) and $z_{\mathrm{cm}}=8.00 \AA$ (middle panel) and their difference (bottom panel). 
("N-down", $\left.\theta=0^{\circ}\right)$, parallel orientation $\left(\theta=90^{\circ}\right)$ and $\mathrm{O}$ headed for the surface ("O-down", $\left.\theta=180^{\circ}\right)$. The azimuth $\phi$ was set to $0^{\circ}$ and the NO bond length was $1.15 \AA$.

After these calculations for the NO/Au energy grid I additionally explored the configurational space of the system. I calculate the energy dependence of both polar angle $\theta$ and azimuth $\phi$ at $z_{\mathrm{cm}}=2.20 \AA$ and for the latter case also at $\mathrm{z}_{\mathrm{cm}}=4.50 \AA$. As the azimuth was changing the molecule was fixed in parallel orientation $\left(\theta=90^{\circ}\right)$. For the parallel orientation I also varied $r_{\mathrm{NO}}$ between 0.8 and $1.8 \AA$ with the molecule at $z_{\mathrm{cm}}=2.2 \AA$, again.

To get also the influence of lattice motion into the PES I did calculations with slab configurations from an equilibration at a temperature of $300 \mathrm{~K}$ with the molecule at different COM distances to the surface ranging from 1.7 to $4.2 \AA$, random orientations and a bond length between 0.9 and $1.8 \AA$. Furthermore, the configurations of the MEP of the NO dissociation on $\mathrm{Au}(111)$, as found in section 6.3, were calculated. Since IESH does not only need the PES of the electronic ground state but also a representation for the electronic excited state, all calculations described in the last paragraph were also done with an electric field of $0.1 \mathrm{eV} \AA^{-1}$ applied in positive and negative $z$-directions. Additionally to the energy the charge on the NO molecule was determined for every configuration by using the Bader charge analysis (see 3.4). From the ground state energy, the energy difference between the calculations with positive and negative electric fields applied, and with the charge on NO it is possible to obtain the matrix elements of the diabatic Hamiltonian for every configuration following the procedure in [157].

In Fig. 43 the results of the calculations done here using RPBE are compared to the results of the calculations done by RoY et al. using PW91 [157]. When the molecule approaches the surface the results with PW91 show that the molecule is stabilized at the surface, whereas the energy profile calculated with RPBE shows no well depth when NO is close to the surface (see panel a). This is due to the different functionals because RPBE is known to underestimate adsorption energies. The charge on NO is more negative at the surface in the case of PW91 than with RPBE (panel c). When NO approaches the surface with $r_{\mathrm{NO}}=1.6 \AA$ both functionals show a decrease in the interaction energy which is much larger for PW91 than for RPBE (panel b), and NO has a more anionic character in the case of PW91 (panel d). In the case of a change of the bond length both functionals seem to agree better in the energies and charges (panels e+g) than in the previous discussed cases.

The change of the molecular orientation to the surface (panel f) shows that the $\mathrm{N}$ down orientation $\left(\theta=0^{\circ}, 0.4 \mathrm{eV}\right)$ is clearly more stable at the surface than the $O$-down orientation $\left(\theta=180^{\circ}, 1.5 \mathrm{eV}\right)$ and the parallel orientation $\left(\theta=90^{\circ}, 0.45 \mathrm{eV}\right)$, too. The stabilization of the $N$-down orientation is also observed in the case of PW91 but it is 

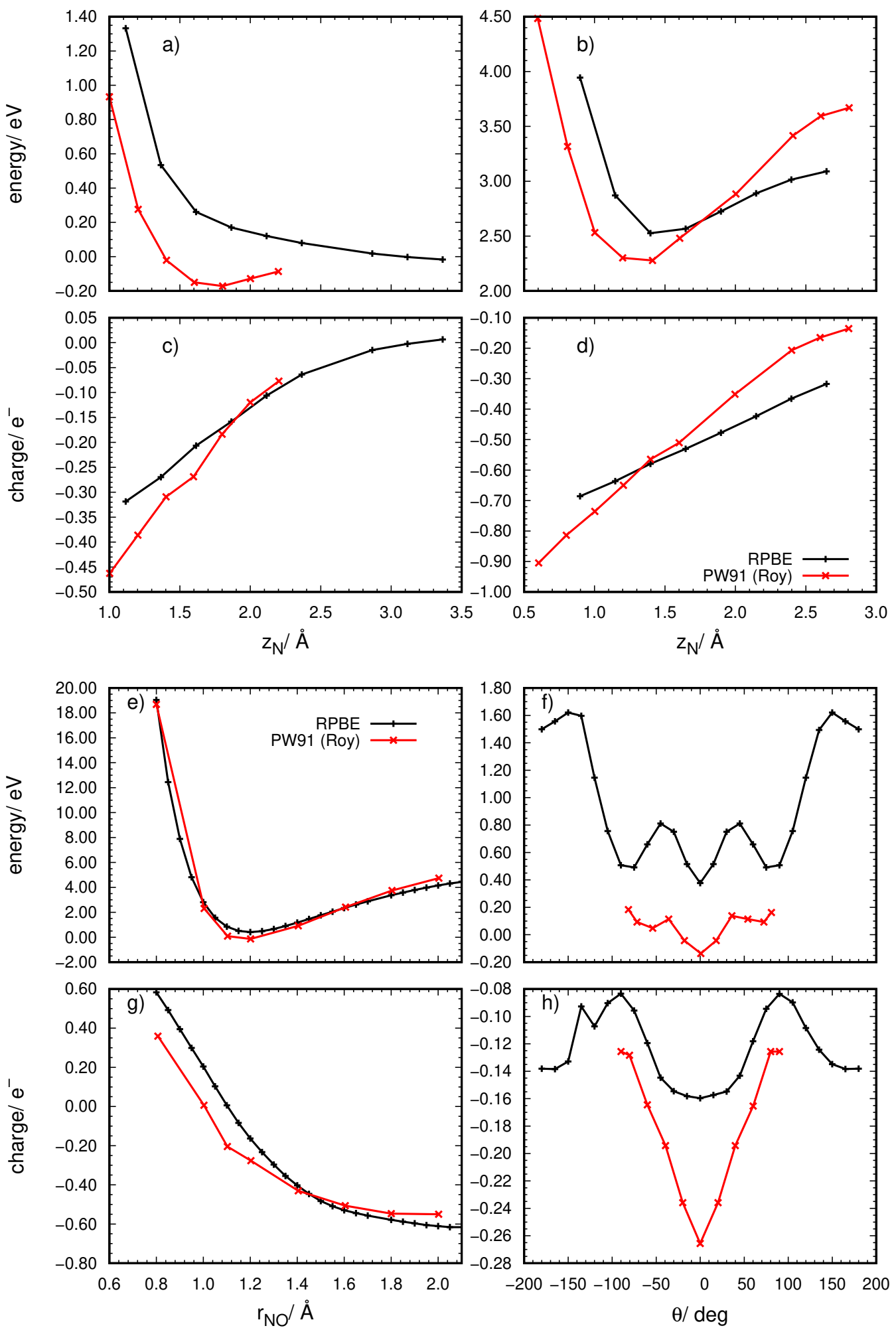

Fig. 43: DFT ground state energies (a,b,e,f) in $\mathrm{eV}$ and charge $(\mathbf{c}, \mathbf{d}, \mathbf{g}, \mathbf{h})$ on the NO molecule in $e^{-}$for NO at the hcp site of $\mathrm{Au}(111)$ for: the $\mathrm{N}$ atom distance to the surface $\left(z_{\mathrm{N}}\right)$ for $r_{\mathrm{NO}}=1.19 \AA$ (a,c) and $r_{\text {NO }}=1.19 \AA(\mathbf{b}, \mathbf{d})$; and NO at $z_{\mathbf{c m}}=2.2 \AA, \theta=90^{\circ}$ for $r_{\text {NO }}(\mathbf{e}, \mathbf{g})$ and the angle $\theta$ between surface normal and $r_{\text {NO }}$. The reference energy is the one of NO at $z_{\mathrm{cm}}=8 \AA$ with $r_{\mathrm{NO}}=1.15 \AA$ and $\theta=0^{\circ}$. The black lines represent calculations with RPBE done in this work and the red lines represent calculations done with PW91 taken from [157]. 
smaller $(0.3 \mathrm{eV})$ and the rotation barrier of the molecule is only around $0.2 \mathrm{eV}$, whereas it is about $0.4 \mathrm{eV}$ in the case of RPBE. A higher rotational barrier was also observed by YIN et al. with $\mathrm{PW} 91$ for $\mathrm{NO} / \mathrm{Au}(111)$; therefore the lower barrier in the calculations done by RoY and co-workers may be caused by lateral image interaction due to the smaller $\mathrm{p}(2 \times 2)$ cell. Finally, the charge transfer to NO is more pronounced in the case of PW91 $\left(0.27 e^{-}\right)$than for RPBE $\left(0.15 e^{-}\right)$when $\mathrm{N}$ is turned to the surface (panel $\left.\mathrm{g}\right)$.

The difference in the charges between this work and the calculations of Tully's group can be due to the difference in the functional or the lateral interactions but it seems to be likely that they are due to differences in the Bader analysis. As said in subsection 7.4.4, a reference file was used in the Bader analysis within this work, but this is not clear in the case of the other work; moreover, this cannot be the case because the possibility to write out the reference file was first introduced in version 4.6.31 [158] of VASP and RoY et al. used the version 4.6.28 [157].

Finally, the DFT calculations performed here are the first step on the route to obtain a new diabatic potential. The next step is to calculate matrix elements of the potential for each set of nuclear configurations and then fit them to obtain the diabatic potential, usable for IESH. The fitting procedure seems to be the most crucial point in the whole process. For this reason, this should be done with neural networks which were successfully used to produce a ground state PES for this system [50]. Furthermore, this PES clearly shows that the problems, with the potential on which IESH simulations for $\mathrm{NO} / \mathrm{Au}(111)$ [46-48] are based, are clearly attributed to the fitting procedure to obtain the potential and not to the DFT data itself, which is also supported by the calculations done here.

\subsubsection{NO molecule in vacuum}

The energetics of the NO molecule was characterized in a simulation box with the same dimensions as for the simulation box for $\mathrm{NO}$ at $\mathrm{Au}(111): \mathrm{p}(3 \times 3)$ cell with 4 layers and a vacuum distance of $20 \AA$. The reciprocal space was sampled first with $4 \times 4 \times 1$ $\boldsymbol{k}$-point grid, but tests with one $\boldsymbol{k}$-point at the $\Gamma$-point showed that the energy difference is around $1 \mathrm{meV}$. The electronic occupation was described by a MP1 function with $\sigma=0.2 \mathrm{eV}$. The geometry optimization was stopped as the forces on the atoms are

$>0.01 \mathrm{eV}^{-1}$. Further, I determined the vibrational frequency of the optimized structure via the finite difference approach. So the same input parameters were used to do the calculations as for the $\mathrm{NO} / \mathrm{Au}(111)$ calculations, which were carefully chosen to get fast convergence in this system. The lone molecule might be better described by another choice of parameters. This is indeed the case because a look at the electronic occupation 
of the orbitals reveals that the unpaired electron is smeared over two orbitals which are degenerated in energy. Thus, for the molecule a wrong electronic structure is obtained, because a splitting of electrons is unreasonable. Choosing a smaller $\sigma=0.01 \mathrm{eV}$ leads to the correct electron distribution over the bands so that no electron smearing is observed. But this incorrect electron distribution does not affect the energy in case of the molecule. To determine the dissociation energy of the molecule $D_{\mathrm{e}, \mathrm{NO}}$, I calculated the energy of either $\mathrm{N}$ and $\mathrm{O}$ atom to get the correct spin-polarized reference and thus

$$
D_{\mathrm{e}, \mathrm{NO}}=E_{\mathrm{NO}}-E_{\mathrm{N}}-E_{\mathrm{O}}
$$

where $D_{\mathrm{e}, \mathrm{NO}}$ is the dissociation energy of NO; $E_{\mathrm{NO}}, E_{\mathrm{N}}$ and $E_{\mathrm{O}}$ are the EwoS from the VASP calculations of the NO molecule and the $\mathrm{N}$ and $\mathrm{O}$ atom, respectively. An overview of all obtained results for the molecule and the atomic species is presented in Tab. 5. A calculation with both atoms $5 \AA$ separated in $z$-direction delivers $E_{\mathrm{N}}+E_{\mathrm{O}}$ within $\mathrm{meV}$ accuracy. So the interaction between the two atoms is negligible at this distance. This can also be concluded from the fact that the energy does not depend on the total spin state. The choice of $\sigma$ is more important, especially for the $\mathrm{O}$ atom. In this case a change of $\sigma$ leads to a change in energy of about $300 \mathrm{meV}$. This is due to the higher electronic entropy in the $\mathrm{O}$ atom. Here the three p-orbitals are just occupied with two electrons which can be distributed in three ways over the orbitals. Hence the electronic entropy is higher and thus a higher $\sigma$ increases the entropic contribution of the energy. In case of either the $\mathrm{N}$ atom or the molecule there is no possibility to distribute the electrons over degenerate energy levels.

\subsubsection{Investigating the Bader analysis using different input files}

To obtain the two diabatic states for $\mathrm{NO}$ and $\mathrm{NO}^{-}$at $\mathrm{Au}(111)$ as input for IESH, it is necessary to calculate, in addition to the ground state energy of the system, the charge on the molecule. This can be obtained from a Bader analysis of the electric charge density of the system, as described in section 3.4. As both, VASP and FHI-aims, write out the electron density I did calculations with both of them to see if the Bader analysis leads to different outcomes, because both codes use different approaches to describe the wave function: PAW approach (VASP) and all-electron approach (FHI-aims). For this purpose, I analyzed the corresponding output files of the electron density, cube files in the case of FHI-aims and CHGCAR files in the case of VASP, for two different configurations, when NO is close $\left(z_{\mathrm{cm}}=1.75 \AA\right)$ and far away $\left(z_{\mathrm{cm}}=8.00 \AA\right)$ from an $\mathrm{Au}(111)$ slab with $\mathrm{N}$-down orientation. The same simulation cell as described above, $\mathrm{p}(3 \times 3)$ slab with 4 layers and $20 \AA$ of vacuum distance in $z$-direction, was used. In case 
Tab. 5: Computational details and results as the EwoS, molecular bond length $d_{\mathrm{NO}}$ and vibrational frequency $\omega_{\mathrm{e}}$ for the NO molecule in vacuum calculated with VASP using RPBE. Further the results for the atomic species to determine the molecular binding energy $D_{\mathbf{e}, \mathrm{NO}} . S$ gives the total spin state of the system.

\begin{tabular}{cccccccc}
\hline System & spin-polarized & $\boldsymbol{k}$-point grid & $\sigma \mathrm{eV}$ & $E / \mathrm{eV}$ & $D_{\mathrm{e}, \mathrm{NO}} \mathrm{eV}$ & $\mathrm{d}_{\mathrm{NO}} / \AA$ & $\omega_{\mathrm{e}} / \mathrm{cm}^{-1}$ \\
\hline \multicolumn{7}{c}{ NO molecule } \\
NO optimized & yes & $4 \times 4 \times 1$ & 0.2 & -11.9163 & -7.2081 & 1.176 & 1895.9 \\
NO optimized & yes & $1 \times 1 \times 1$ & 0.2 & -11.9193 & -7.2102 & 1.176 & \\
NO optimized & yes & $4 \times 4 \times 1$ & 0.01 & -11.9746 & -6.8698 & 1.764 & 1898.0 \\
NO optimized & yes & $1 \times 1 \times 1$ & 0.01 & -11.9781 & -6.8720 & 1.177 & 1897.0 \\
NO optimized & no & $4 \times 4 \times 1$ & 0.2 & -11.6033 & -11.5903 & 1.176 & 1900.9 \\
\hline N + O & yes, S=1/2 & $4 \times 4 \times 1$ & 0.2 & -4.7094 & - & 5.0 & - \\
N + O & yes, S=1/2 & $1 \times 1 \times 1$ & 0.2 & -4.7110 & - & 5.0 & - \\
N + O & yes, S=5/2 & $4 \times 4 \times 1$ & 0.2 & -4.7093 & - & 5.0 & - \\
N + O & yes, S=1/2 & $4 \times 4 \times 1$ & 0.01 & -5.1056 & - & 5.0 & - \\
N + O & yes, S=1/2 & $1 \times 1 \times 1$ & 0.01 & -5.1071 & - & 5.0 & - \\
\hline N atom & yes, S=3/2 & $4 \times 4 \times 1$ & 0.2 & -3.1564 & - & - & - \\
N atom & yes, S=3/2 & $1 \times 1 \times 1$ & 0.2 & -3.1565 & - & - & - \\
N atom & yes, S=3/2 & $4 \times 4 \times 1$ & 0.01 & -3.1565 & - & - & - \\
N atom & yes, S=3/2 & $1 \times 1 \times 1$ & 0.01 & -3.1569 & - & - & - \\
N atom & no & $4 \times 4 \times 1$ & 0.2 & -0.0039 & - & - & - \\
\hline O atom & yes, S=2/2 & $4 \times 4 \times 1$ & 0.2 & -1.5518 & - & - & - \\
O atom & yes, S=2/2 & $1 \times 1 \times 1$ & 0.2 & -1.5526 & - & - & - \\
O atom & yes, S=2/2 & $4 \times 4 \times 1$ & 0.01 & -1.9483 & - & - & - \\
O atom & yes, S=2/2 & $1 \times 1 \times 1$ & 0.01 & -1.9492 & - & - & - \\
O atom & no & $4 \times 4 \times 1$ & 0.2 & -0.0091 & - & - & - \\
\hline
\end{tabular}


of VASP the same computational details as stated at the end of subsection 7.4.1 were used. For FHI-aims similar details as given for this code at the beginning of section 7.4 were used, except that the basis sets were described by tight settings.

Tab. 6: Bader charges for different ways of analysis. Shown are the following: the number of electrons on the $\mathrm{N}$ atom $\mathrm{N}_{\mathrm{elec}, \mathrm{N}}, \mathrm{O}$ atom $\mathrm{N}_{\mathrm{elec}, \mathrm{O}}$, NO molecule $\mathrm{N}_{\mathrm{elec}, \mathrm{NO}}$ and the total number of electrons in the system $\mathrm{N}_{\text {elec,tot }}$ of the Bader analysis and its given value for two different configurations of $\mathrm{NO} / \mathrm{Au}(111)$ system. In the bottom panel the $x-, y-, z$-coordinates of the $\mathrm{N}$ and $\mathrm{O}$ atoms are given, respectively.

\begin{tabular}{|c|c|c|c|}
\hline Methods & FHI-aims & CHGCAR & CHGCAR with reference \\
\hline \multicolumn{4}{|c|}{$\mathrm{NO}\left(t o p, z_{\mathrm{cm}}=1.75 \AA\right)$} \\
\hline $\mathrm{N}_{\text {elec,N}}$ & 6.693 & 4.309 & 4.119 \\
\hline $\mathrm{N}_{\text {elec }, \mathrm{O}}$ & 8.295 & 6.449 & 6.449 \\
\hline $\mathrm{N}_{\text {elec,NO }}$ & 14.988 & 10.758 & 10.568 \\
\hline $\mathrm{N}_{\text {elec,tot }}(\mathrm{bader})$ & 3430.45 & 407.0000 & 407.0000 \\
\hline $\mathrm{N}_{\text {elec,tot }}$ & 2874 & 407 & 407 \\
\hline \multicolumn{4}{|c|}{$\mathrm{NO}\left(t o p, z_{\mathrm{cm}}=8.00 \AA\right)$} \\
\hline $\mathrm{N}_{\text {elec,N }}$ & 6.650 & 3.703 & 4.471 \\
\hline $\mathrm{N}_{\text {elec }, \mathrm{O}}$ & 8.547 & 7.238 & 6.470 \\
\hline $\mathrm{N}_{\text {elec,NO }}$ & 15.197 & 10.941 & 10.941 \\
\hline $\mathrm{N}_{\text {elec,tot }}(\mathrm{bader})$ & 3430.367 & 407.0000 & 407.0000 \\
\hline $\mathrm{N}_{\text {elec,tot }}$ & 2874 & 407 & 407 \\
\hline \multicolumn{4}{|r|}{$z / \AA$} \\
\hline \multicolumn{2}{|c|}{$\mathrm{N}\left(\right.$ top,$\left.z_{\mathrm{cm}}=1.75 \AA\right)$} & \multicolumn{2}{|r|}{1.137} \\
\hline \multicolumn{2}{|c|}{$\mathrm{O}\left(t o p, z_{\mathrm{cm}}=1.75 \AA\right)$} & \multicolumn{2}{|r|}{2.287} \\
\hline \multicolumn{2}{|c|}{$\mathrm{N}\left(t o p, z_{\mathrm{cm}}=8.00 \AA\right)$} & \multicolumn{2}{|r|}{7.387} \\
\hline \multicolumn{2}{|c|}{$\mathrm{O}\left(\right.$ top,$\left.z_{\mathrm{cm}}=8.00 \AA\right)$} & \multicolumn{2}{|r|}{8.537} \\
\hline
\end{tabular}

The results of the Bader analysis are represented in Tab. 6. For VASP the analysis was done in two ways, with and without a reference file, as described in section 3.4. There are differences between the different analyses with the CHGCAR file, for the number of electrons on the two atoms for both configurations. But this affects the total charge on the molecule only, when the molecule is close to the surface. The difference to FHIaims is clearly due to the all-electron characteristic in case of this code. An interesting observation in the case of FHI-aims is that the Bader analysis does not give the total number of electrons for the system $\left(7 e^{-}+8 e^{-}+36 \times 79 e^{-}=2874 e^{-}\right)$but a value which is much larger ( $\left.3430 e^{-}\right)$. This is due to the core electrons (inner shell) which are employed in FHI-aims. Because of them a very fine grid, on which the electron density is written, is needed. But even for a very fine grid is was not possible to obtain more reliable results and to use finer grids is not feasible due to memory considerations. However, a detailed analysis of this is far beyond the scope of this thesis and so this point will not further be discussed. However, the Bader analysis was not used to obtain the charge 
for $\mathrm{CO}$ and the slab atoms from the FHI-aims output to determine the transition dipole moment (see chapter 9). Finally, the VASP results suggest that the use of a reference file leads to a more reasonable result for the charge on the individual species. Furthermore, this is recommended by the Henkelman group [158] who developed the Bader analysis code. Therefore, the charge of the NO molecule to get the diabatic states of NO/Au(111) was obtained with this approach.

\subsection{Conclusions}

The energy grid calculations for $\mathrm{CO}$ at $\mathrm{Au}(111)$ reveal a well depth only when the molecule is C-down oriented towards the slab. Neither the parallel nor the O-down orientation of the molecule show attraction when the molecule approaches the surface. The preferred adsorption site of $\mathrm{CO}$ on $\mathrm{Au}(111)$ is the top site, at which the adsorption energy is about $0.2 \mathrm{eV}$ lower than for the two hollow ( $f c c$ and $h c p$ ) and the bridge sites. The PW91 functional gives a deeper attraction well than RPBE. Employing the vdW correction leads to a stronger chemisorption as well as physisorption. Despite slight differences in the actual values for the interaction energy, the overall appearance of the energy grids calculated with VASP and FHI-aims is quite similar. With these calculations it was possible to define the distance of closest approach of CO to the surface, which was used to explain the vibrational relaxation behavior of $\mathrm{CO}$ at $\mathrm{Au}(111)$, via a possible transient formation of $\mathrm{CO}^{-}$at the surface, as reported in [42]. For the interaction between $\mathrm{CO}$ and $\mathrm{Ag}(111)$ as well as for Ag-covered $\mathrm{Au}(111)$ surface we can conclude that the energy grids look very similar to each other and show a more repulsive behavior when the molecule comes close to the surface than in case of $\mathrm{Au}(111)$. Furthermore, the differences in the interaction energy for the Ag-covered surfaces are too small to explain the experimentally observed trend in the translational energy inelasticity. Thus, another effect must exist which would explain this. One reasonable explanation would be the differences in the phonon spectra of the different surfaces as explained in chapter 5 and reported by STEINSIEK et al. [41].

The investigation of the interaction energy of NO and $\mathrm{Au}(111)$ is more complicated due to the problems in DFT with open-shell systems, i.e. spin-polarized calculations. These convergence problems especially occur when the energy of the molecule far away from the surface is calculated. This is especially seen in the case of VASP when the magnetic moment is allowed to relax without setting the initial moments of the species. The problems are clearly reduced if the initial moments are set. If the total magnetic moment of the system is fixed the calculations seem to converge to a wrong spin state when the molecule is far away from the surface, because the energy is higher than the 
energy calculated when the total moment is relaxed (see Fig. 36). Calculations with FHI-aims give results which are comparable to those of VASP when the initial magnetic moments are set. However, the parameters found in subsection 7.4.1 for VASP seem to deliver converged results for $\mathrm{NO} / \mathrm{Au}(111)$ and were used to produce DFT data which can be used to obtain a diabatic potential. Furthermore, the charge on the NO molecule has to be determined via the Bader analysis. For this purpose, tests of this analysis with both codes and in the case of VASP with and without a reference file were done (see subsection 7.4.4). It turns out that the Bader analysis of the FHI-aims output provide doubtful results, especially with respect to the total number of electrons within the system, whereas the analysis of the VASP output provides the expected outcome.

The calculated DFT energies and the charges from the Bader analysis are in qualitative agreement with those of Roy et al. [157]. The differences are mainly due to the employed XC functionals (RPBE in this work and PW91 in the work by ROY), the cell size and the Bader analysis. In a further perspective the obtained data can be used to follow the route proposed by TULLY and co-workers $[47,157]$ to construct a diabatic potential to make it possible to perform IESH simulations for this system. This means to calculate the elements of the diabatic Hamiltonian from the DFT data and fit the data with an appropriate procedure. The best choice seems to be the neural network approach [49] which was successfully applied to construct a ground state PES for this system [50], but also a more conventional approach similar to that used by RoY et al. [157] can be chosen. A comparison of both approaches would be interesting as it would answer the following questions: does the fitting procedure lead to the bad results of IESH; or are bad converged DFT data responsible for this. 


\section{AIMD simulations for scattering of CO and NO from (111) metal surfaces}

\subsection{Introduction}

In this chapter I am going to describe AIMD simulations of scattering $\mathrm{CO}$ and $\mathrm{NO}$ from different metal (111)-surfaces. These simulations were performed to see if they can describe the scattering experiments done with $\mathrm{CO}$ in different vibrational states [39, 40, 42, 43] from (111) metal surfaces and NO from $\mathrm{Au}(111)$ [20, 21, 44] and $\mathrm{Ag}(111)$ [159]. Since the AIMD simulations are based on the BOA, one could see to which extent the energy transfer between the molecule and the surface is dominated by non-adiabatic effects. Thus, if the results of the simulations are in agreement with those of the experiments the role of these effects could be negligible in the molecule-surface interaction, because non-adiabatic effects seem to be more prominent in the encounter of a highly vibrationally excited molecule with a metal surface. Simulations within the BOA are very interesting, and in the case of a low vibrationally excited molecule the experiment and simulation should agree well or at least to a high extent, whereas in the case of the highly vibrationally excited molecules simulated and experimental results should reveal large discrepancies. So by carrying out AIMD simulations of scattering experiments of $\mathrm{CO}$ (and NO) in different vibrational states and by comparing the obtained results with the experiment we can find out to which extent the molecule-surface interaction is dominated by non-adiabatic effects, like ehp excitation. Furthermore, the simulations can provide data which can be used as input for a fitting procedure to get a PES for the investigated system.

The presented simulations comprise different initial conditions of the molecule as translational energy and different initial vibrational states but as well as different surface temperatures. First, I will show the results of CO scattering from Au(111) for different surface conditions; here the molecule is in the vibrational ground state. Then I go on to present the results for $\mathrm{CO}$ scattering from $\mathrm{Au}(111), \mathrm{Ag}(111)$ and Ag-covered $\mathrm{Au}(111)$ surfaces with the molecule in low vibrational states. After that I will get into the simulations of highly vibrationally excited CO scattered from the pure surfaces. To obtain 
these last results tests were done with VASP and FHI-aims which will also be described in section 8.4. In the last section (8.5), I will cover the results of the simulations done with NO in a highly vibrationally excited state from $\mathrm{Au}(111)$ and $\mathrm{Ag}(111)$.

\section{2 $\mathrm{CO}(v=0)$ from $\operatorname{Au}(111)$}

After I did the calculations to investigate the $\mathrm{CO} / \mathrm{Au}(111)$ interaction described in section 7.2, I did AIMD simulations for $\mathrm{CO}(v=0)$ with an initial translational energy of $1.0 \mathrm{eV}$ and normal incidence $\left(\theta=0^{\circ}\right)$ with slabs equilibrated to different temperatures to see how surface temperature influenced the outcome of these scattering simulations. Further these first simulations are done to test if the preparation procedure works well and gives reasonable results. It has to be checked if the random number generator used for polar angle $\theta$, azimuth $\phi$, and $\mathrm{x}_{\mathrm{cm}}$ and $\mathrm{y}_{\mathrm{cm}}$ works well when the molecule initial configuration above the surface is generated. Secondly, it should be checked if the procedure to equilibrate the slab to a certain temperature, to mimic a surface at this temperature, is reliable. The procedure to describe the preparation of the slab equilibration and the molecule in its initial state was described in section 4.2. To carry out the actual equilibration simulations the following input parameters were used: the spin-unpolarized approach, with the corresponding XC-functional (RPBE or PW91); the electronic occupation of bands was modeled with a MP1 smearing and a $\sigma=0.2 \mathrm{eV}$, and a $4 \times 4 \times 1 \boldsymbol{k}$-point grid sampled the reciprocal space and a cut-off energy of $400 \mathrm{eV}$ for the plane waves was used. The calculations within the scf-cycles were carried out with the fast algorithm ( $A L G O=F)$. Furthermore, a time step of $0.5 \mathrm{fs}$ and convergence criterion for the scf-cycle of $10^{-5} \mathrm{eV}$ were used. The slab atom positions were initially described by their equilibrium lattice constants of $4.20 \AA$ for RPBE and $4.165 \AA$ for PW91, respectively.

Since energy calculations of the $\mathrm{CO} / \mathrm{Au}(111)$ system, see section 7.2 , show that the interaction between molecule and surface is small at $\mathrm{z}_{\mathrm{cm}}=5.5 \AA$ for RPBE and PW91 this was used as initial the $z$-coordinate of the COM of the molecule. The bond length was equal to $1.148 \AA$ and $1.142 \AA$ for RPBE and PW91, respectively, which was the optimized bond distance for $\mathrm{CO}$ in a simulation box with the same dimensions as a $\mathrm{p}(3 \times 3)$ slab with 4 layers and a vacuum distance of $20 \AA$. The simulations were done with the RPBE functional for a slab were all atoms where fixed on their equilibrium positions, for a slab where the three uppermost layers were allowed to move, and for a slab where atoms of those layers were equilibrated at $150 \mathrm{~K}$ and to $300 \mathrm{~K}$. For these simulations similar input parameters as for the slab equilibration were used (see above), except for the simulations with the rigid slab in which a higher convergence criterion of $10^{-3} \mathrm{eV}$ for 
the change in total energy (EDIFF) was used. This leads to a too large drift in energy of $50 \mathrm{meV}$. Thus, for all other simulations a convergence criterion of $10^{-5} \mathrm{eV}$ was used. The trajectories were ended after 1 ps or were stopped when the COM of the molecule was more than $5.5 \AA$ away from the slab. For the first three sets (rigid slab, $T_{\mathrm{s}}=0 \mathrm{~K}, 150 \mathrm{~K}$ ) 50 trajectories were run, whereas for the highest temperature $\left(T_{\mathrm{s}}=300 \mathrm{~K}\right) 100$ trajectories were computed to get better statistics for comparison to the available experimental data.

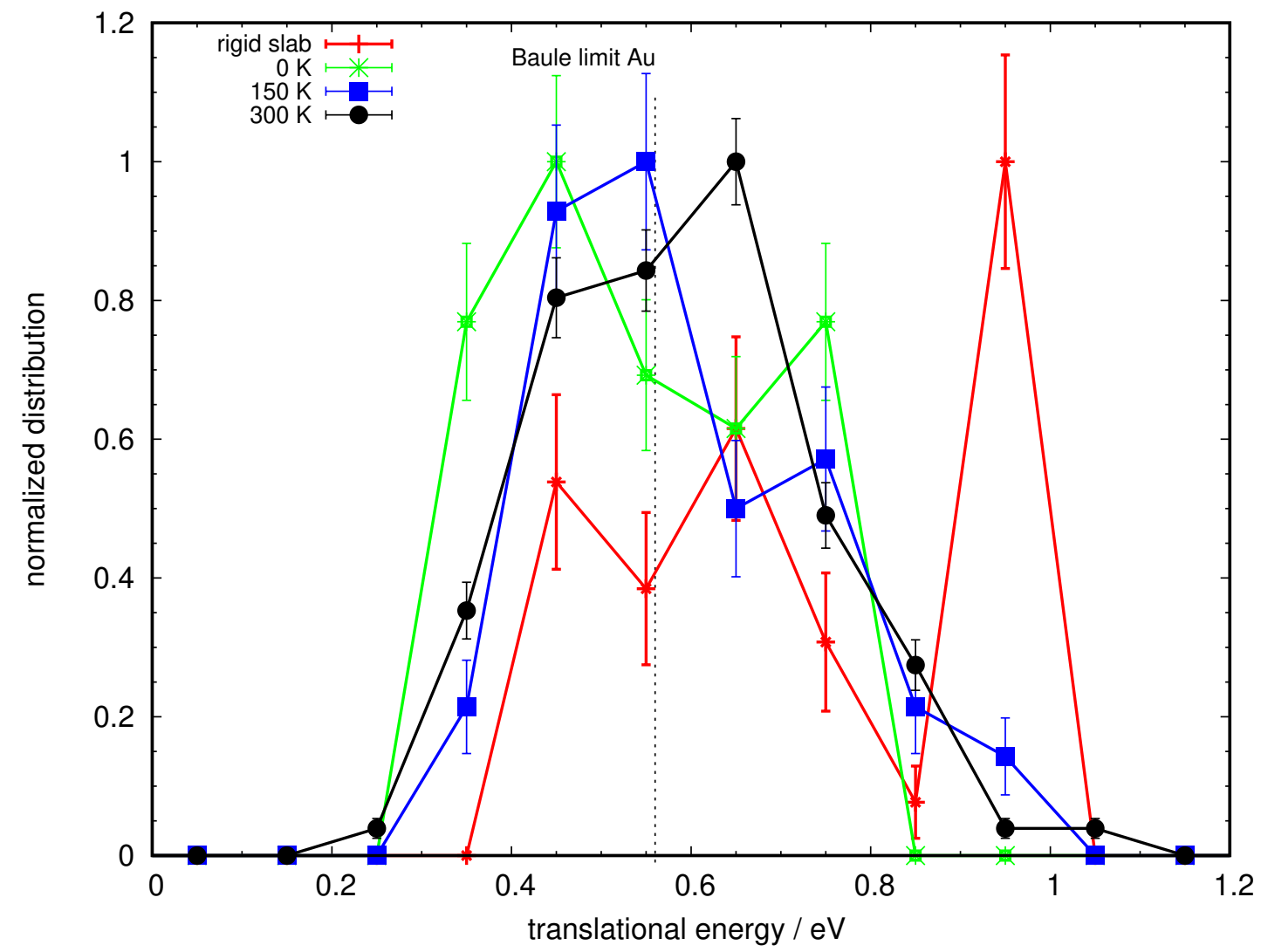

Fig. 44: Translational energy distribution (normalized to the maximum) for $\operatorname{CO}(v=0)$ scattered from $\mathrm{Au}(111)$ with an initial translational energy of $1 \mathrm{eV}$ at normal incidence $\left(\theta=0^{\circ}\right)$. The distributions are indicated by red crosses (rigid slab), green crosses $\left(T_{\mathbf{s}}=0 \mathrm{~K}\right)$, blue $\left(T_{\mathbf{s}}=150 \mathrm{~K}\right)$ squares and black circles $\left(T_{\mathrm{s}}=300 \mathrm{~K}\right)$. To generate the distribution a binning of $0.1 \mathrm{eV}$ was used. The error bars indicate a confidence interval of $95 \%$. The black dotted vertical line indicates the Baule limit of CO and one Au atom.

A comparison of the translation energy distributions for the different slab temperatures shows that an increase of the temperature leads to a broader distribution. This can be learned from Fig. 44. The error bars used in here as well as in the following, were calculated with the statistical uncertainty

$$
\Delta=\frac{\sqrt{\frac{N_{\text {traj,bin }}}{N_{\text {traj }}}\left(1-\frac{N_{\text {traj,bin }}}{N_{\text {traj }}}\right)}}{\sqrt{N_{\text {traj }}}},
$$


where $N_{\text {traj,bin }}$ is the number of trajectories with the binning and $N_{\text {traj }}$ is the total number of trajectories included in the distribution. The actual error bars are defined by $2 \Delta$ to include $95 \%$ of the events within the bars. Furthermore, there is a clear difference between molecules scattered from a movable and from a rigid slab. The energy distribution for the latter case shows that a large fraction of the scattered trajectories retains most of the initial translational energy, whereas in the case of movable atoms the molecules lose a large fraction of their initial energy. This shows the importance of the energy dissipation into the surface dofs.

When the positions of the slab atoms are fixed the only possibility for the molecule to lose translational energy is to redistribute it within itself, i.e. to transfer it to rotational or vibrational dof. This happens for a part of the trajectories scattered from the rigid surface which lose a large amount of their initial energy, as can be recognized in Fig. 44 . To see in which molecular dofs the energy is redistributed during the scattering event one can correlate the final energies of the different dofs with each other. The final energies of the molecule and the slab were determined as described in section 4.3.

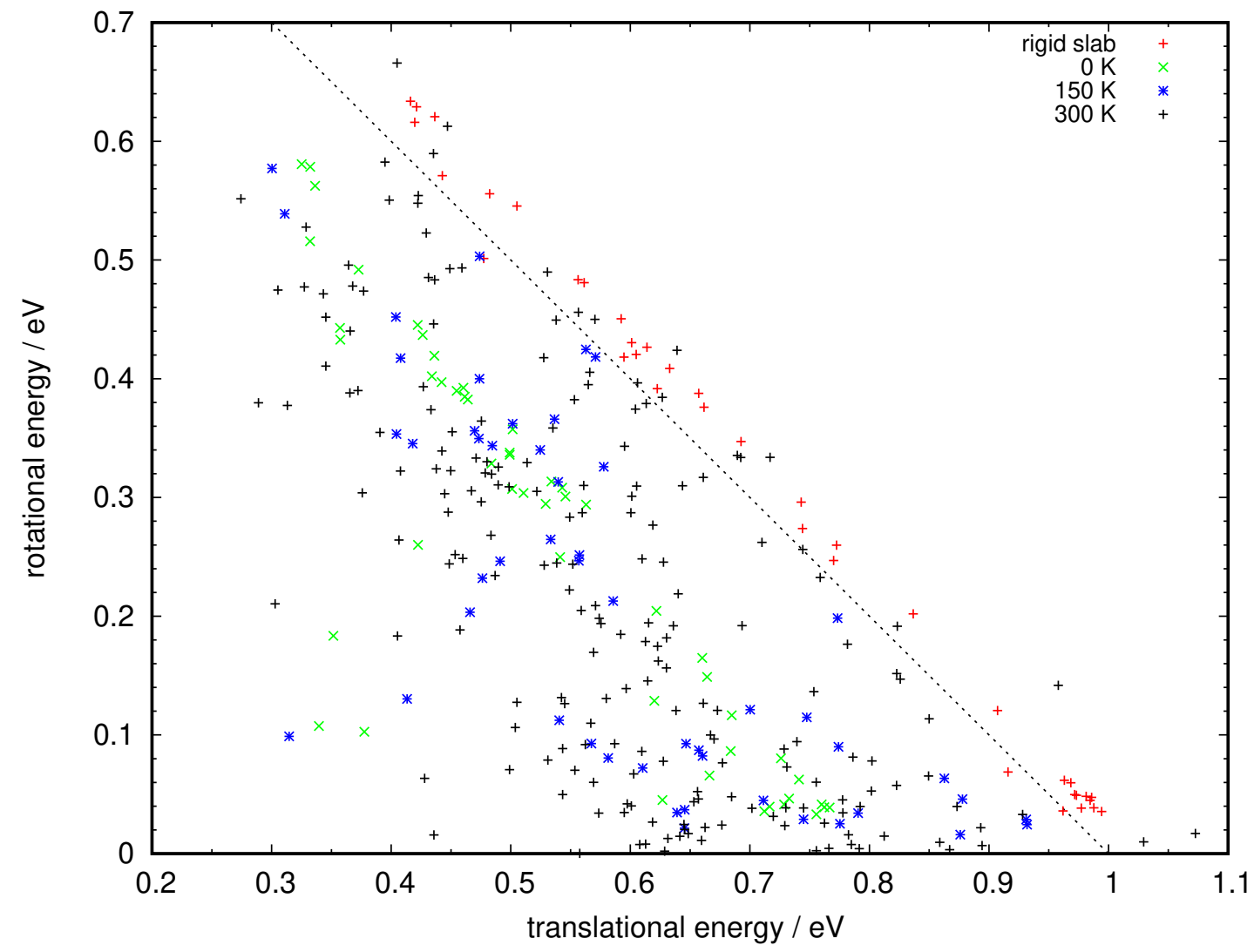

Fig. 45: The final rotational energy $\left(R_{f}\right)$ vs. the final translational energy $\left(T_{f}\right)$ for different slab conditions is shown. The red crosses represent the rigid slab, the green ones the slab at $0 \mathrm{~K}$, the blue stars the slab at $150 \mathrm{~K}$ and the black crosses the slab at $300 \mathrm{~K}$. The dotted line indicates the total conversion of translational into rotational energy (total anti-correlation). 
The correlation between the molecular translational energy $T_{f}$ and the rotational energy $R_{f}$ is shown in Fig. 45. The figure clearly reveals a strong anti-correlation between translation and rotation. For the simulations with a rigid slab (red points) this anticorrelation can be well described by a line with a slope of -1 , but the points are slightly shifted due to the total energy drift.

So the tail of the CO translational energy distribution in case of the rigid surface in Fig. 44 can be seen as a rotational rainbow [20, 160], and this feature smears out when the surface temperature is increased. Therefore, the anti-correlation between rotational and translational energy is stronger for the slab with a temperature of $0 \mathrm{~K}$ than for the slabs with higher temperatures where the translational energy is more broadened.

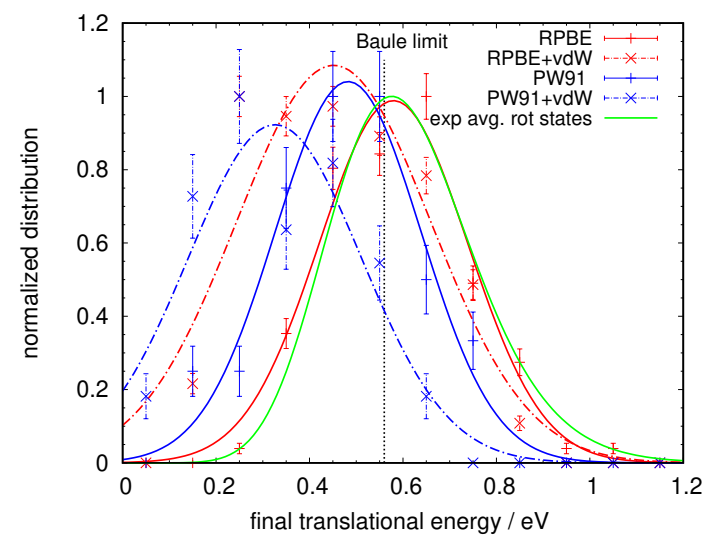

Fig. 46: Final translational energy distribution of simulations of $\operatorname{CO}(\nu=0)$ scattered from $\operatorname{Au}(111)$ at $300 \mathrm{~K}$ for RPBE (red crosses), RPBE with vdWcorrections (red stars), PW91 (blue crosses) and PW91 with vdW-corrections (blue stars). The green line represents a fit to the experimental data summed overall rotational states and weighted with the corresponding Boltzmann factors.

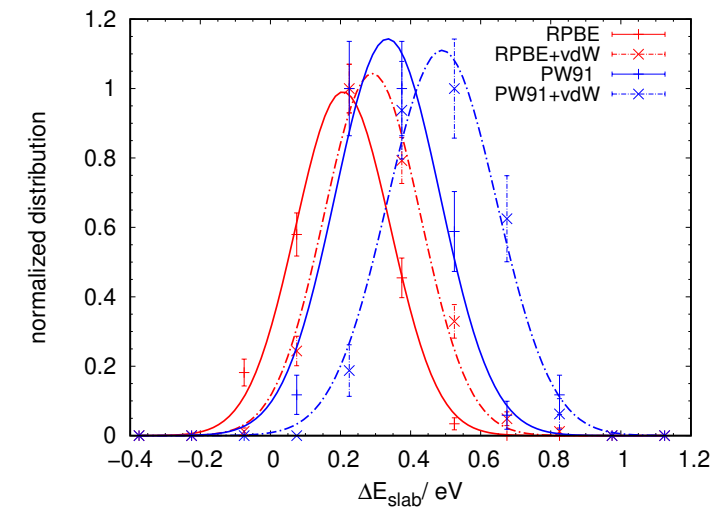

Fig. 47: Distribution of the energy transfer to the slab energy $\left(\Delta \mathbf{E}_{\text {slab }}\right)$ distribution of simulations of $\mathrm{CO}(\nu=0)$ scattered from $\mathrm{Au}(111)$ at $300 \mathrm{~K}$ for RPBE (red crosses), RPBE with vdW-corrections (red stars), PW91 (blue crosses) and PW91 with vdW-corrections (blue stars). A negative value indicates the loss of energy of the slab.

For a surface temperature of $300 \mathrm{~K}$ I also did simulations with the PW91 functional and investigated the influence of vdW interaction for RPBE and PW91 to see which functional provides the best description of the experimental findings. The translational energy distribution for different functionals and the experiment is shown in Fig. 46. It turns out that the translational energy distribution obtained with the RPBE functional describes the experimental one best. The distributions obtained with PW91 as well as with both functionals including vdW corrections are clearly shifted to lower translational energies with respect to the experimental results (private communication with $\mathrm{K}$. Golibrzuch). Thus, the functionals with vdW-corrections result in distributions which show stronger energy losses than their counterparts without corrections. This is to be 
expected because the stronger interaction between molecule and surface when the long-range vdW interactions are taken into account.

That the RPBE functional delivers the best description of the experimentally obtained final translational energy distribution was not to be expected, since considering just the results from the calculations of the interactions energies between molecule and surface the well-depth of the functional is far away from the experimentally determined well-depth, see section 7.2. However, the deeper well-depths in the molecule-surface interaction potentials calculated with vdW-corrections and PW91, as shown in Fig. 34, may suppose that the higher loss in translational energy for trajectories calculated with these corrections is attributed to these deeper well-depths. Thus, in these cases the translational energy of the molecule is transferred to the dofs of the slab and not internally distributed to rotation and vibration within the molecule. This can be seen from Fig. 47, where for the distribution of the final slab energy with respect to the initial one is represented. Here the trajectories which show a large loss of their initial translational energy show a strong gain of slab energy so that the order of functionals in the slab energy distribution is ad vice versa as to the translational energy one. In simulations no scattered molecule shows a gain in the vibrational energy higher than $100 \mathrm{meV}$, which means no vibrational excitation from the ground state is observed. So, the intra-molecular energy exchange between translational and vibrational dofs is small. This is seen for the different slab temperatures as well as for the different functionals. But since just a relatively small number of trajectories was calculated a vibrational excitation is not to be expected, because the experimentally determined vibrational excitation probability from the ground state to the first excited state is around $10^{-4}$ [40], so that 1 of 10000 molecules is excited. And here only a relatively small number of 200 trajectories was calculated. Moreover, the typical relaxation time for an $T-R$ energy transfer is about $10^{4}$ faster than the one of $T$ - $V$ transfer and therefore no vibrational excitation is observed.

\section{3 $\mathrm{CO}(v=2)$ from $\mathrm{Au}(111), \operatorname{Ag}(111)$ and $\mathrm{Ag}$-covered $\operatorname{Au}(111)$}

Since scattering experiments of $\mathrm{CO}(\nu=2)$ from pure and silver-coated $\mathrm{Au}(111)$ surfaces were done by STEINSIEK et al. [39] I did AIMD simulations to mimic those experiments. One reason for this was that the experimental results seem to be explainable via a purely mechanical model, that means without non-adiabatic effects. Thus, the differences in the final translational energy distributions for the different (111) surfaces are explained 
by differences in the Debye frequencies of the surfaces. However, non-adiabatic effects do not seem to play a role during the encounter between the molecule and the surface. If this is the case AIMD simulations should deliver very similar outcomes compared to the experimental ones.

For these simulations the molecule needs to be vibrationally excited, i.e. the molecule needs to get assigned the initial values of potential $U(r)$ and kinetic vibrational energy $T_{\text {vib }}$ as described in subsection 4.2.1. To construct the potential vibrational energy curve for a CO molecule I performed DFT calculations with RPBE functional of its energy for various values of the inter-nuclear distance and fitted these data with a Morse potential. The data and the fit can be seen in Fig. 48. The calculation was done in a simulation box with the same dimensions as for the simulations done with RPBE in the previous section (8.2), and the atoms were moved in the $z$-direction. The other control parameters were similar to those used before, except that the spin-polarized approach was used with an initial magnetic moment (MAGMOM) of 2 for $\mathrm{C}$ and -2 for $\mathrm{O}$, to account for the unpaired spins when the atoms are far away from each other.

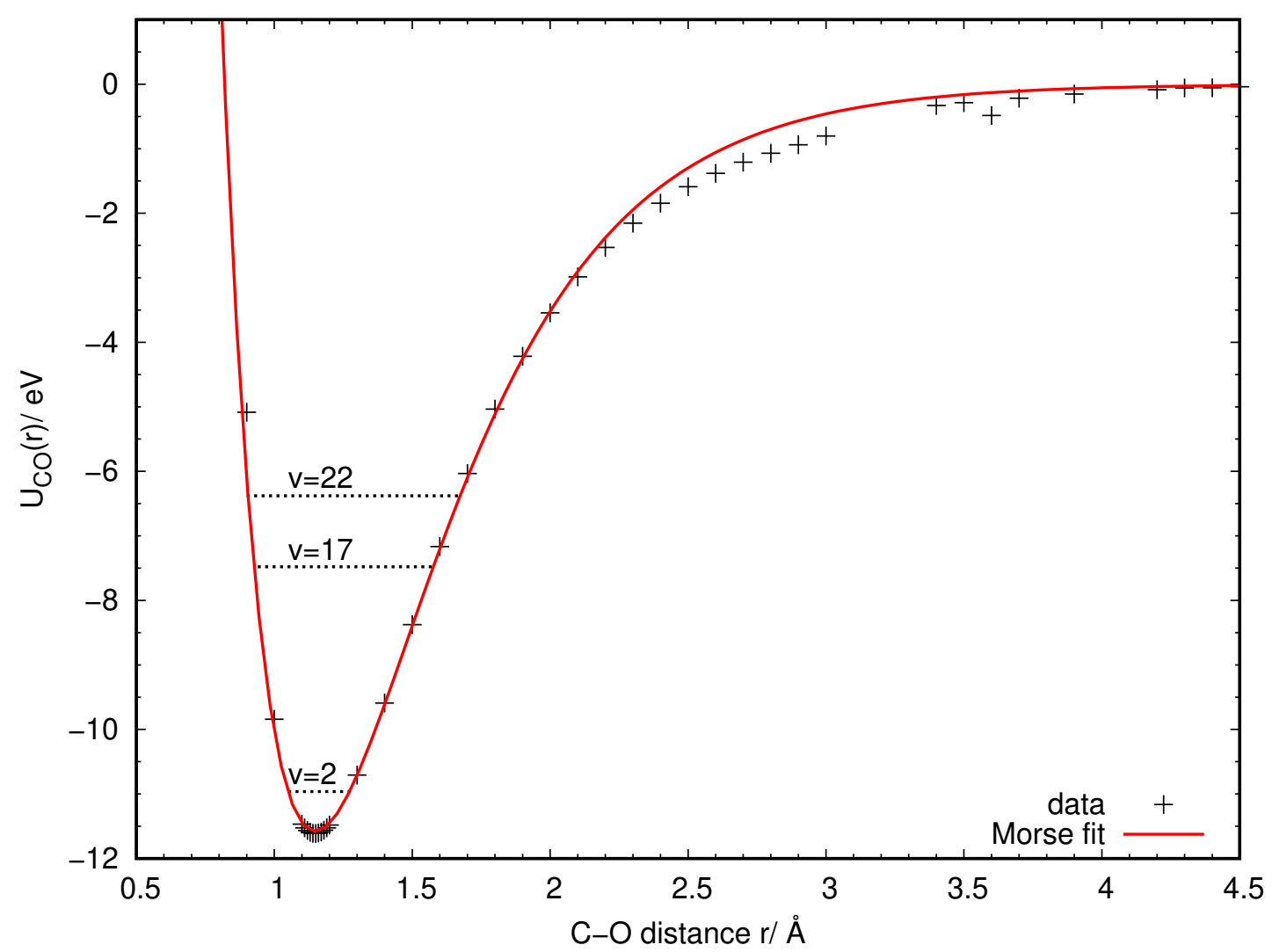

Fig. 48: Potential energy of the CO molecule calculated with RPBE as a function of the bond distance (black crosses) and a Morse fit through the data (red line). The dotted black lines indicate the initial vibrational states for which simulations for $\mathrm{CO}$ were carried out. 
The resulting parameters of the Morse potential are: equilibrium bond length $r_{\mathrm{eq}}=$ $1.148 \AA$, the well depth $D_{\mathrm{e}}=11.579 \mathrm{eV}$ and the stiffness $\alpha=2.115 \AA^{-1}$. The total vibrational energy corresponding to $v=2$ was found to be $0.618 \mathrm{eV}$ and the initial translational energy was $0.6 \mathrm{eV}$.

How I built up the Ag-covered $\mathrm{Au}(111)$-slabs was described in detail in section 5.2. The $5 \mathrm{p}(3 \times 3)$ slabs with 4 layers were all described by the same optimized lattice constant of $4.20 \AA$ determined with RPBE. The slabs were equilibrated to $300 \mathrm{~K}$, using the procedure described in section 4.2, to match the experimental surface temperatures. Also, the computational set up for these AIMD simulations was similar to the one used for the previous simulations with CO in the vibrational ground state: VASP with the RPBE functional in the spin-unpolarized framework, an MP1 smearing with a width of $0.2 \mathrm{eV}$, and a $4 \times 4 \times 1 \boldsymbol{k}$-point grid for the reciprocal space was used. Furthermore, plane waves up to an energy of $400 \mathrm{eV}$ were included. The scf-cycle was stopped when the energy difference between two steps was smaller than $10^{-5} \AA$.

In the top panel of Fig. 49 the final translational energy distributions are shown for the AIMD simulations for $\mathrm{CO}(v=2)$ scattered from a $\mathrm{Au}(111), \mathrm{Ag}(111)$ and $\mathrm{Au}(111)$ covered with 1, 2 and 3 ML silver. Clearly, the molecules scattered back from the Au(111) slab lose less of their initial translational energy as the Ag-covered slabs. When the number of Ag-layers is increased the fits of the energy distributions come closer to the pure silver case (green dashed line).

This trend can also be seen in the bottom panel of Fig. 49 where the mean final translational energy is given as function of the silver layer thickness for simulation and the experiment. Although there is a difference between simulation and experimental values, as the mean final energy of the simulations goes up from 1 ML to 2 ML Ag whereas experimental goes down, the overall trend is the same. Thus, the mean energy values decrease when going from pure gold to a pure silver surface and we can say that simulations and experiment are in qualitative agreement with each other. The error bars (which give $2 \times$ the standard deviation) of the calculations are relatively large and thus the experimental data lay in the range of uncertainty of the simulations. When we correct the data of the simulations by about a constant value of $0.075 \mathrm{meV}$, simulated and experimental data overlap very well. The differences between the experiment and the simulations of about $0.075 \mathrm{eV}$ may be due to the used XC-functional (RPBE) as it is known to underestimate adsorption well depths, which then causes the lower translational energy loss with respect to experiment. Additionally, the experimentally dosing of the $\mathrm{Au}(111)$-surface with more than three mono-layers of silver does not lead to changes in the obtained mean final translational energy. 

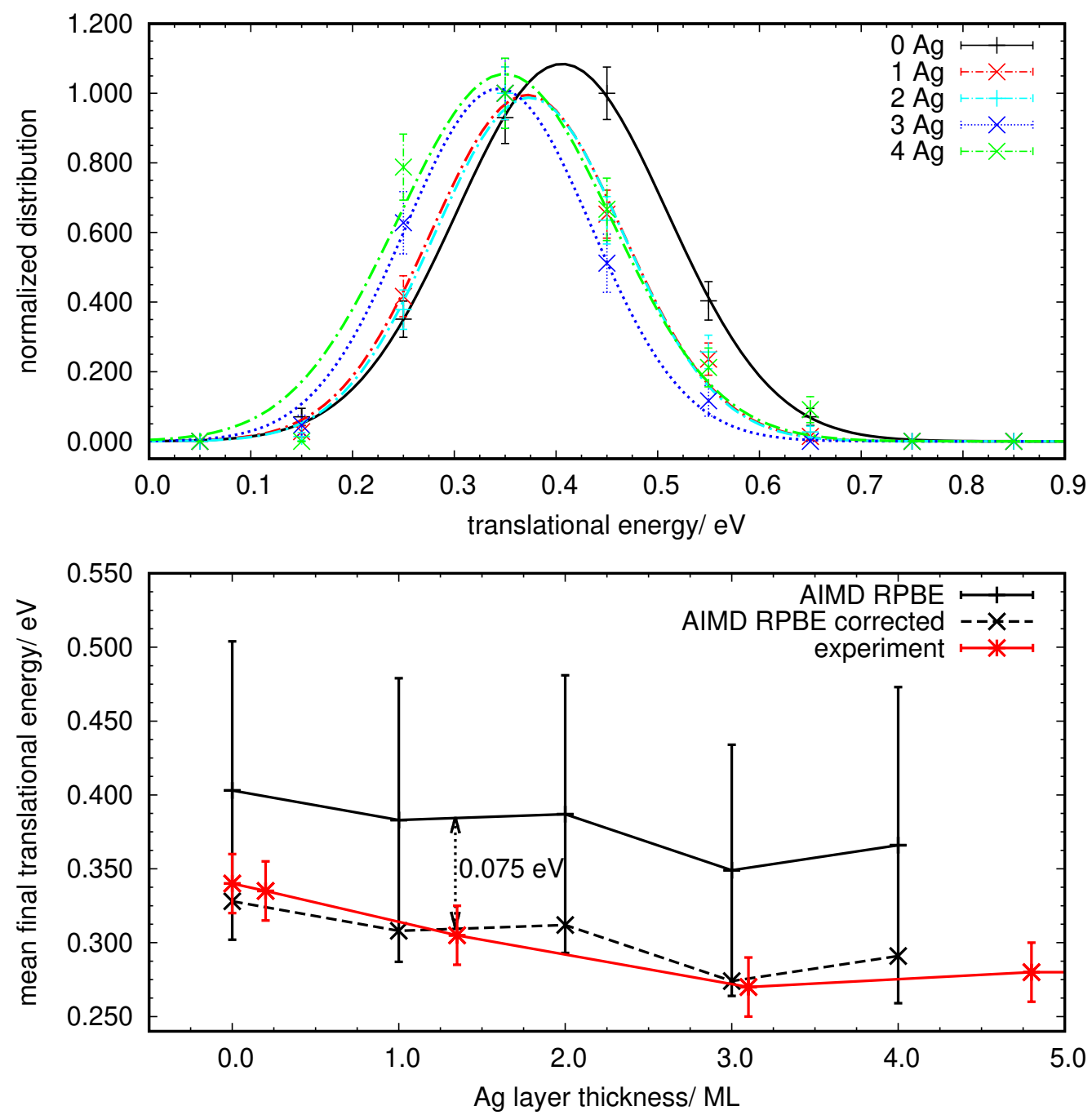

Fig. 49: Normalized (to maximum signal) final translational energy distribution for $\mathrm{CO}\left(\nu=2, E_{\mathrm{in}}=\mathbf{0 . 6} \mathrm{eV}\right)$ scattered from Au(111) (black), Ag(111) (dashed green) and Ag-covered Au(111) slabs with 1 (dashed red), 2 (dashed torquois) and 3 layers Ag (dashed blue) (top panel). Mean final translational energy vs. the Ag layer thickness for the AIMD (black crosses) and experimental (red stars) results (bottom panel). 

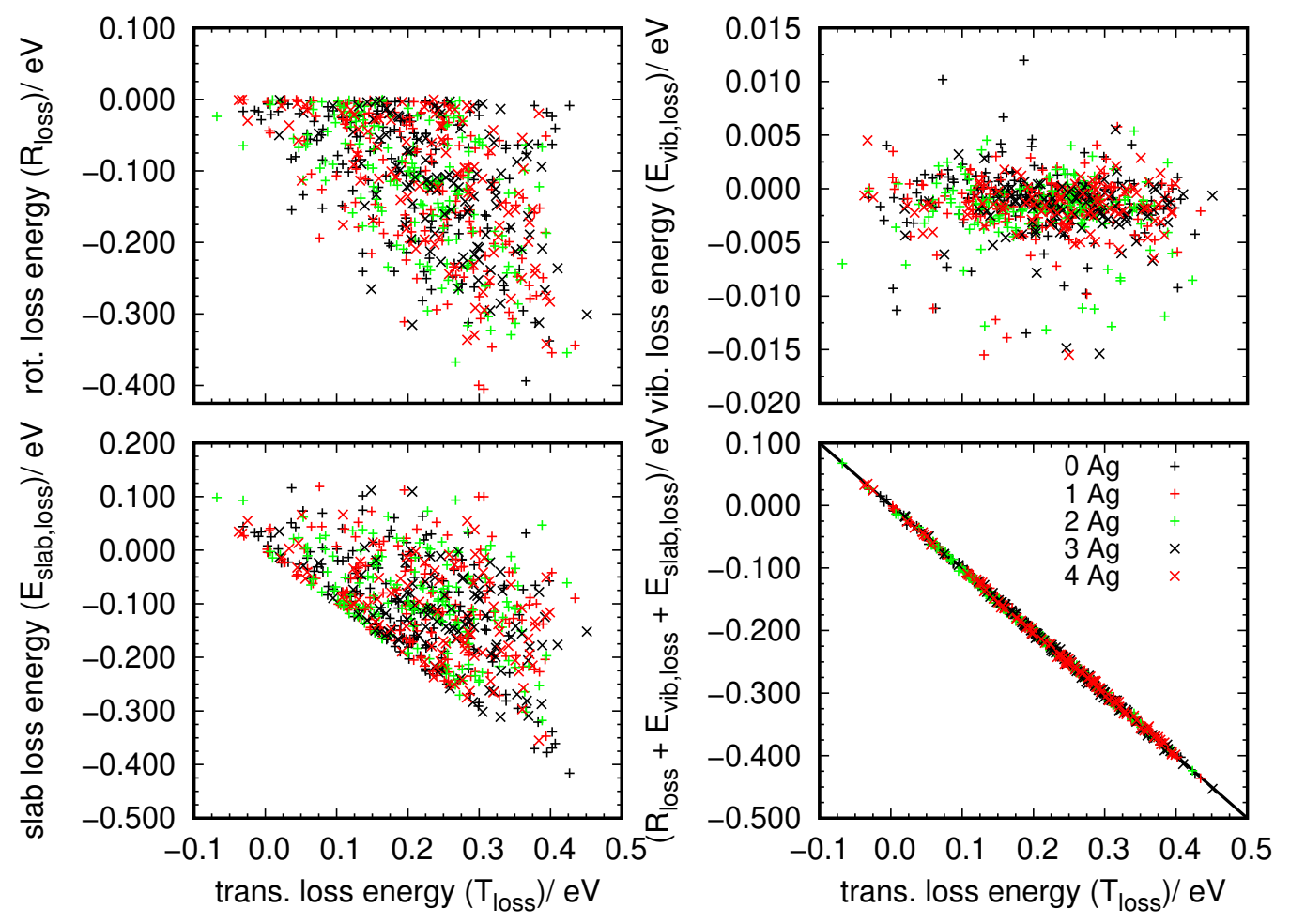

Fig. 50: Translational energy loss plotted against the rotational (top left), vibrational (top right), and slab (bottom left) energy loss for $\mathrm{CO}(v=2)$ scattered from $\mathrm{Ag}(111)$ and Ag-covered $\mathrm{Au}(111)$ slabs. In the bottom right $T_{\text {loss }}$ is plotted against the sum of the other three energies, here the solid black line indicates a total anti-correlation should guide the eyes . 
The initial translational energy of the projectile is mainly transferred to the rotational and into the slab dofs. This can be seen in Fig. 50 where the translational energy loss ( $T_{\text {loss }}$ ) is plotted against three different dofs of the system. In the bottom right, the conversion between the sum of $R_{\text {loss }}, E_{\text {vib,loss }}$, and $E_{\text {slab,loss }}$ and $T_{\text {loss }}$ shows that the translational energy of the molecule is nearly completely converted into one of the three former energies, which is supported by the fact that all trajectories lie close to a straight line with a slope of -1 , which is represented by a black solid line.

For all computed trajectories no strong loss or gain of molecular vibrational energy is seen. The vibrational energy loss or gain is under $0.020 \mathrm{eV}$ for all scattered trajectories. Therefore, we can conclude that no vibrational relaxation of $\mathrm{CO}$ during the collision with the different slab is observed, because for this a stronger loss in vibrational energy, at least half of a vibrational quanta $(0.1 \mathrm{eV})$, has to occur. As the experimental vibrational relaxation probability of $\mathrm{CO}(v=2)$ scattered from $\mathrm{Au}(111)$ and Ag-covered $\mathrm{Au}(111)$ surfaces is $>0.01$ [41], it seems to be plausible that the simulations show no relaxation as the number of trajectories for every system calculated here is between 100 and 175 . From these results I conclude that it is possible to describe the scattering of $\mathrm{CO}$ in low vibrational states from $\mathrm{Au}(111)$ and Ag-covered $\mathrm{Au}(111)$ surfaces by AIMD simulations based on the BOA. Thus, the energy transfer can be completely described by classical mechanical arguments, i.e. the energy is transferred between nuclear dofs of the projectile and the slab atoms on a single PES. Hence the system remains in its electronic ground state over the whole simulation time.

\section{4 $C O(v=17,22)$ from $\operatorname{Au}(111)$ and $\operatorname{Ag}(111)$}

\subsubsection{Simulations using VASP}

Using a similar setting as for the simulations of $\mathrm{CO}$ scattering in low vibrational states (see section 8.3) displayed a strong drift in the total energy in the case of CO with higher vibrational energy (above $2 \mathrm{eV}$ ). This was observed for a trajectory with CO having a total energy of $7 \mathrm{eV}$, distributed between translational $(1 \mathrm{eV})$ and the vibrational $(6 \mathrm{eV}$ dofs. $\mathrm{CO}$ was scattered from an $\mathrm{Au}(111) \mathrm{p}(3 \times 3)$ cell with 4 layers where all slab atoms were kept fixed. The time step was set to 0.1 fs due to the faster vibrational motion of the molecule.

Fig. 51 shows the potential and kinetic energies of the system and their sum, thus the total energy, as function of time. In the short time of $100 \mathrm{fs}$ the total energy has some smaller jumps of about $0.05 \mathrm{eV}$ which are already too large and unsatisfactory, but even worse three very large jumps (larger than $0.1 \mathrm{eVs}$ ) are observed. All of these jumps are 
clearly due to jumps in the potential energy of the system, whereas the kinetic one shows an oscillating behavior as expected for a vibrationally excited molecule. The higher and smaller jumps in energy lead to an overall energy drift of $1.3 \mathrm{eV}$ in $95 \mathrm{fs}$ which is ten times larger as a tolerable drift over a trajectory of $2 \mathrm{ps}$.

To investigate the problematic energetic behavior I ran a trajectory with the molecule only in the simulation box. For this purpose I used an input setting very similar to that of the simulations with the Au slab, but I reduced the number of $\boldsymbol{k}$-points to 1 enough for only the molecule in the simulation box. The energies of the molecule are presented in Fig 52. Here the total energy (black line) increases nearly linearly with the simulation time. This increase is due to relatively small jumps of about a few meV, which occur regularly, as indicated by the dotted black lines in Fig. 52 . The jumps seem to occur always when the CO bond length has a value of around $1.45 \AA$.

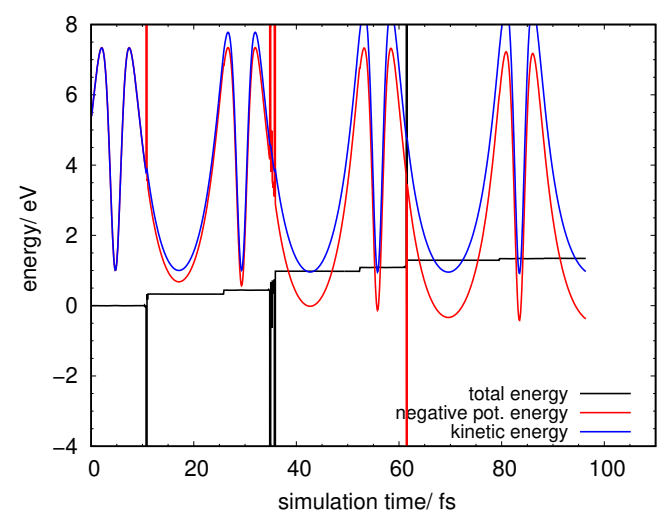

Fig. 51: Time evolution of different energies for a trajectory of $\mathbf{C O}\left(T_{\text {in }}=1 \mathrm{eV}, E_{\text {vib }}=6 \mathrm{eV}\right)$ approaching a rigid $\mathrm{Au}(111) \mathbf{p}(3 \times 3)$ slab with 4 layers. Shown are the total energy drift (black), kinetic energy (blue), and negative potential energy (red line) of the system.

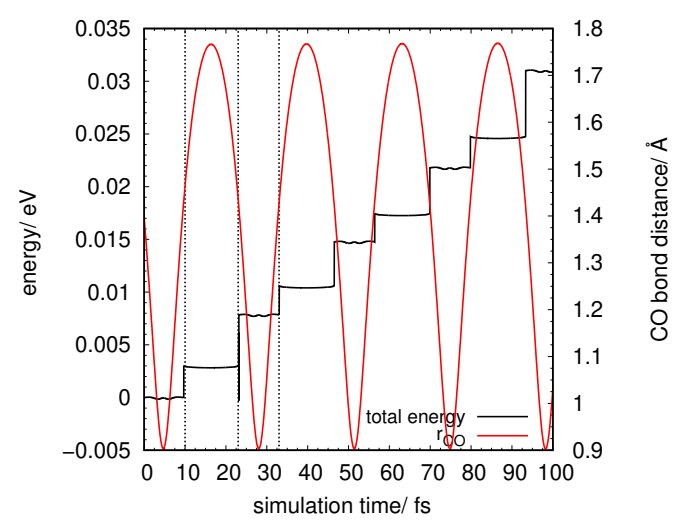

Fig. 52: Trajectory of $\mathbf{C O}\left(T_{\mathrm{in}}=1 \mathrm{eV}, E_{\mathrm{vib}}=6 \mathrm{eV}\right)$ in a simulation cell which has the same dimensions as the cell with the Au slab in it. Shown are the total energy (black) and the CO bond length (red line). The vertical dashed black lines indicate jumps in the total energy.

First, I did tests with a variety of input settings. While keeping the energy convergence criterion to $10^{-6} \mathrm{eV}$ I varied the time step, the algorithm, and the minimum number of scf-cycles (NELMIN). I also used the possibility to fix the Hamiltonian in the beginning for a few electronic cycles, just optimizing the wave function and then optimizing expectation value of the energy with the pre-optimized wave function (NELMDL). The maximum number of electronic steps (NELM) was set to 200. But unfortunately, this did not lead to a significant improvement of the energy conservation.

As the problems with convergence of DFT calculations occurs at the specific CO bond distance of $1.45 \AA$, it is not necessary to do AIMD but rather to calculate the potential energy of $\mathrm{CO}$ for the bond distance in question. I calculated the potential energy for different bond lengths between 0.9 and $6.0 \AA$ with three different ways of spin treatment 
whereby I particularly sampled the region between 1.4 to $1.5 \AA$. First I did unrestricted calculations without constraints of the magnetic moment. Secondly I constrained the latter to zero (NUPDOWN=0) and thirdly I did these calculations in the restricted case. For the two former I set the magnetic moment to 2 on the $\mathrm{C}$ atom and to -2 on the $\mathrm{O}$ atom (MAGMOM-tag). Additionally, I also did calculations using the same spin treatment with FHI-aims.

In Fig. 53 the potential energy difference between the different treatments and the final magnetic moment of the system of $\mathrm{CO}$ in this range of the bond length are shown. So when the two atoms are separated the restricted calculations lead to an incorrect energy, because both atoms have a spin which is not zero. Although the total spin can be zero this is not captured by the restricted calculations. Whereas the differences in the interaction energies for the different spin handling is obvious, the difference between the two programs with the same spin handling is very small. For VASP the potential energy of the unrestricted

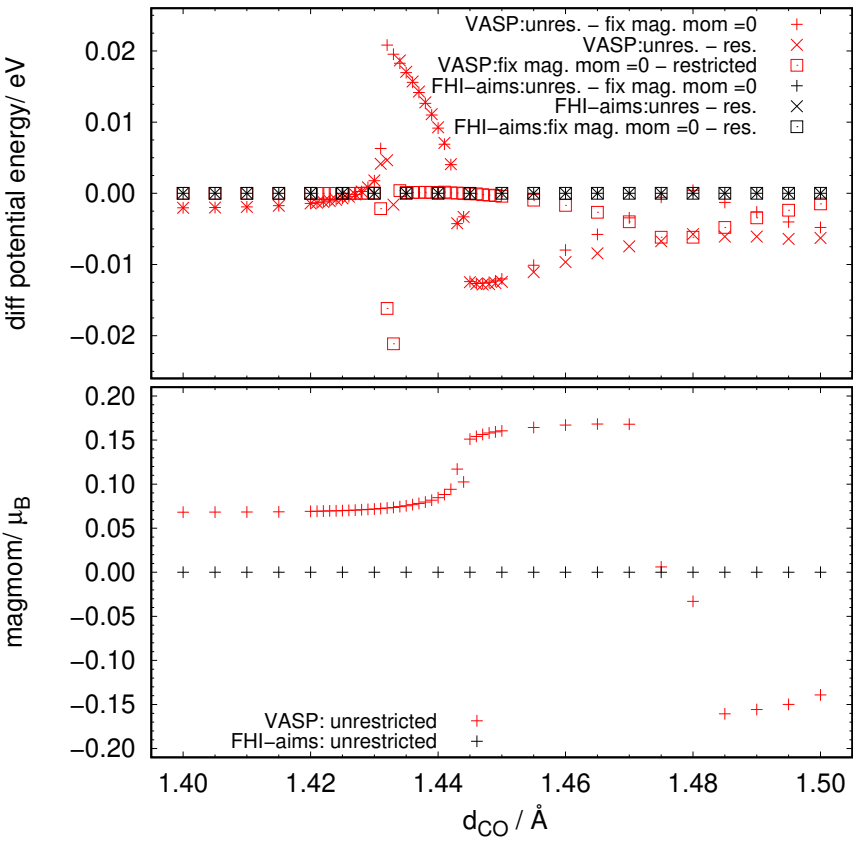

Fig. 53: The upper panel shows the difference in the potential energy between the different cases of the spin treatment (unrestricted, fixed spin, and restricted) for VASP and FHI-aims. The bottom panel shows the magnetic moments of the unrestricted cases without fixed moment, because for these other cases it is 0 .

case without constraints of the magnetic moment clearly differs from the two other cases. Especially around a bond length of $1.44 \AA$ the deviation is up to $20 \mathrm{meV}$, but also for the other distances the potential energy differences differ slightly. This is due to a magnetic moment which is not zero but is around $0.06 \mu_{\mathrm{B}}$ at $1.4 \AA$ and increases to $0.18 \mu_{\mathrm{B}}$ when $d_{\mathrm{CO}}$ is larger than $1.44 \AA$.

So now, an answer to the question what causes the jumps in the total or rather the potential energy in this small bond length range is found. This is an incorrect spin state to which the calculations converge. In the case of restricted calculations the correct state cannot be reached because it has a magnetic moment different to zero. Thus, using the spin-restricted approach for simulations of highly vibrationally excited 
CO seems to be not reasonable. So the next step I did was to calculate a trajectory with vibrationally excited CO within the spin-unrestricted approach of the GGA. These calculations reduced the drift in the total energy but show a magnetic moment not equal to 0 , similar to the magnetic moment in the static calculation, as shown in the bottom panel of Fig. 53.

To deal with this issue one can constrain the magnetic moment in VASP in two ways: keep the total magnetic moment of the system constant or constrain the moment of each atom by means of specifying the magnetic moment of each species in all three directions in space, i.e. specification of the magnetic moment via a vector for each species. The latter approach is a non-collinear one [161]. Here, a penalty term $E_{\mathrm{p}}$ is added to $E_{0}$ to get the total DFT energy $E$, and it gets big when the difference between the actual and the desired magnetic moment is large. An additional factor $\lambda$ can be adjusted to make the penalty term large (small $\lambda$ ) or very small (large $\lambda$ ). After the restriction of the total magnetic moment of the system via NUPDOWN does not lead to the correct spin state, I used the non-collinear for further test calculations.

After performing extensive tests with VASP, which did not solve the problem of the jumps in the total energy of the system in a feasible manner, I decided to tackle the issue with FHI-aims. The calculations with this code are described in the next subsection.

\subsubsection{Simulations with FHI-aims}

From the calculation of the energy for different CO bond distance with FHI-aims, as shown in Fig. 53, we see the same values of potential energy for three different kinds of spin treatment. The magnetic moment in the case of the unrestricted (without constraints for the spin moment) calculation is zero for all bond distances. This is also observed for a larger range of the bond distance. Therefore, it seems to be reasonable that simulations with FHI-

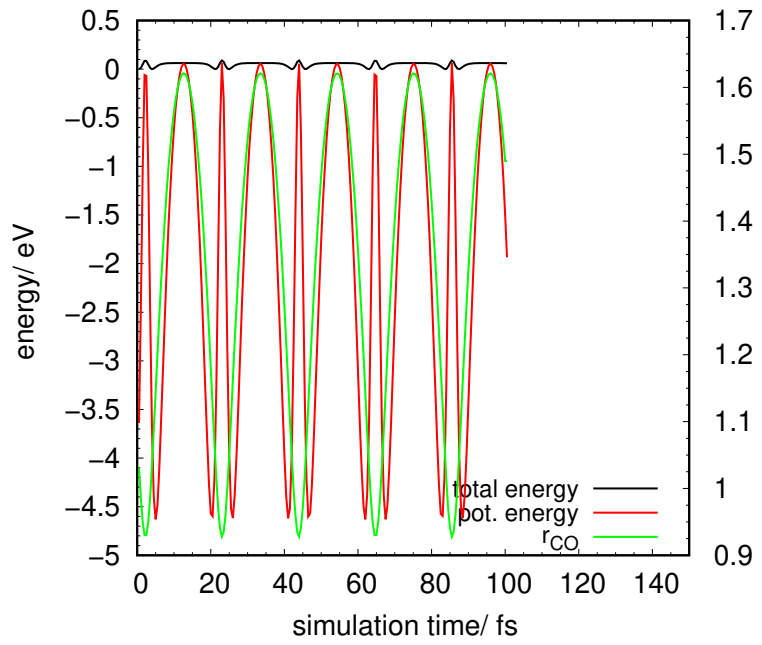

Fig. 54: Total (black) and potential energies (red) as well as the bond distance (green) for $\mathrm{CO}(=17)$ in vacuum along the AIMD trajectory. aims should give better total energy conservation than VASP. This is indeed the case, as shown in Fig. 54, where the 
total, kinetic and potential energies of the $\mathrm{CO}$ molecule along a trajectory are plotted. The calculations are done with the following input setting: RPBE functional within the spin-unpolarized approach, Gaussian smearing function $(\sigma=0.2 \mathrm{eV}), 1 \boldsymbol{k}$-point, and light settings for the basis sets. The $s c f$-cycle was stopped when forces acting on the atoms were $<1 \times 10^{-4} \mathrm{eV} \AA^{-1}$. Further a time step of $0.5 \mathrm{fs}$ was chosen. The simulation cell has the same dimensions as the one with a $\mathrm{p}(3 \times 3)$ slab with 4 layers and vacuum distance of $20 \AA$. The total energy of the trajectory computed with FHI-aims shows clearly no drift, only oscillations with small amplitudes about $0.08 \mathrm{eV}$. These peaks occur when the potential energy shows a maximum which is the one of the inner turning point of the potential. This can be concluded from the bond distance (green line in Fig. 54) which shows its minimum value when the peaks in the total energy appear. Further tests showed that the height of the peaks could be reduced by using a smaller time step. After succeeding in minimizing the energetic drift for simulations with the molecule I did tests with an $\mathrm{Au}(111)$ surface again. These tests were done with similar input parameters as described for the molecule. Since the surface was modeled by $\mathrm{p}(3 \times 3)$ cell with 4 layers $\left(a_{0}=4.20 \AA\right.$ ), a $4 \times 4 \times 1 \boldsymbol{k}$-point grid was sufficient to sample the reciprocal space. Convergence for the scf-cycle was set to $10^{-6} \mathrm{eV}$ with respect to a change in the total electronic Hamiltonian and to $10^{-5} \mathrm{eV} \AA^{-1}$ for a change of the forces acting on every atom. As basis sets for the included species the light setting was used. A time step of $0.1 \mathrm{fs}$ was used, which gave good results for the molecule in vacuum. The initial positions and velocities of the slab atoms were obtained by equilibrating to $300 \mathrm{~K}$, the positions of the bottom layer were kept fixed as for the VASP calculations, and the initial positions and velocities of the molecule were generated as described in section 4.2.1, using an incidence energy of $1 \mathrm{eV}$ and a vibrational energy of $2 \mathrm{eV}$. So the $E_{\mathrm{vib}}$ was set to a bit lower value then it was set in previous calculations, because the corresponding slower oscillations may reduce the drift in total energy.

The total, potential and kinetic energies of the system along the AIMD trajectory are shown in Fig. 55a. At the beginning of the trajectory the drift in total energy is clearly smaller than in case of the VASP trajectory. But when the molecule approaches the surface the total energy increases up to $0.1 \mathrm{eV}$ and even more when the molecule comes back from the surface. Although there is a significant drift in total energy, no jumps are observed in the potential energy and in the kinetic energy, as it was in the VASP calculations (see Fig. 51). In spite of this encouraging observation the drift in total energy is rather large and has to be further reduced to achieve the aim of reliable results for these AIMD simulations.

Since the drift in the total energy seems clearly be due to the presence of the surface some adjustments with respect to it may provide the reduction in the energy drift. As 


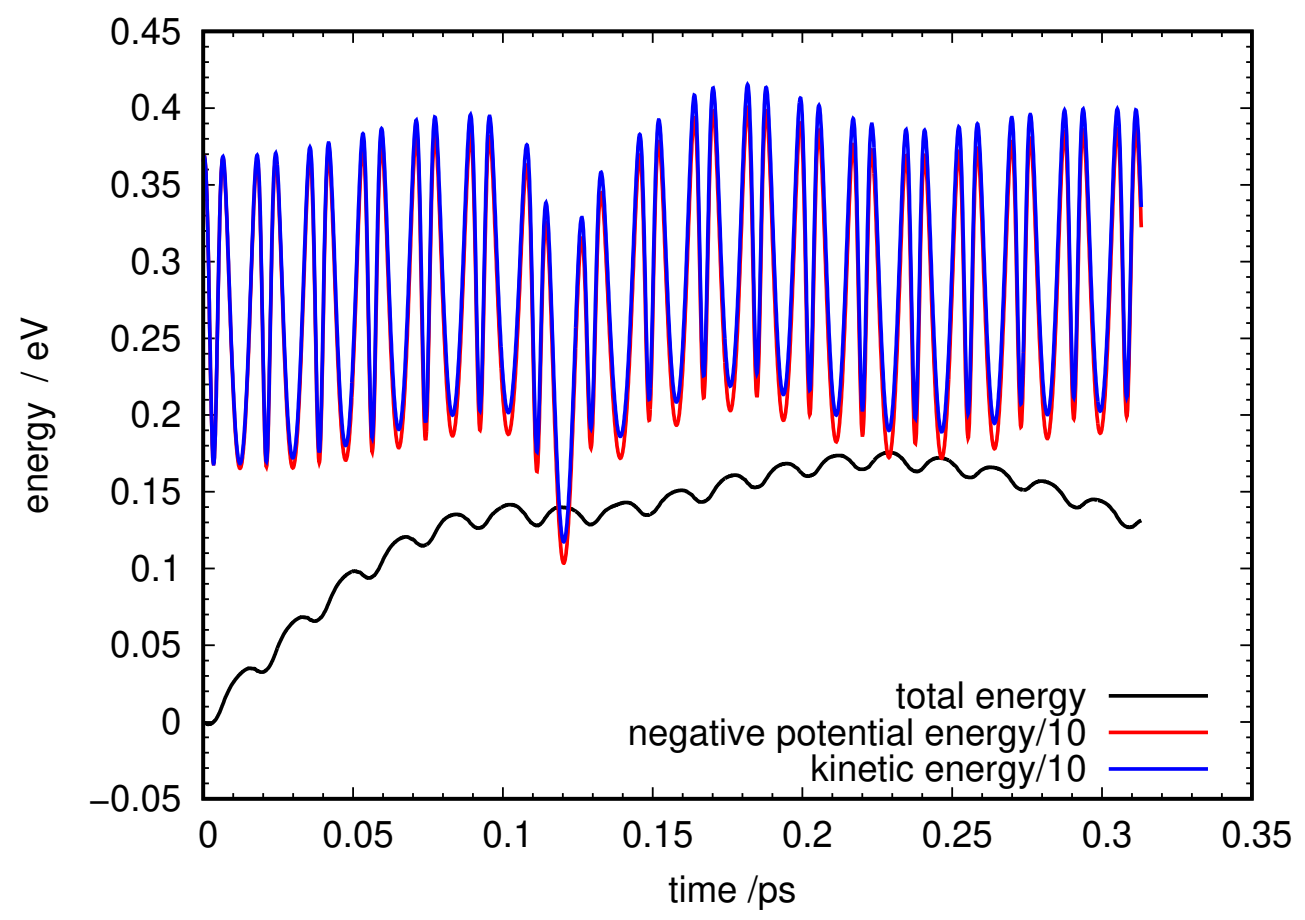

(a) $p(3 \times 3)$-slab with a rigid bottom layer.

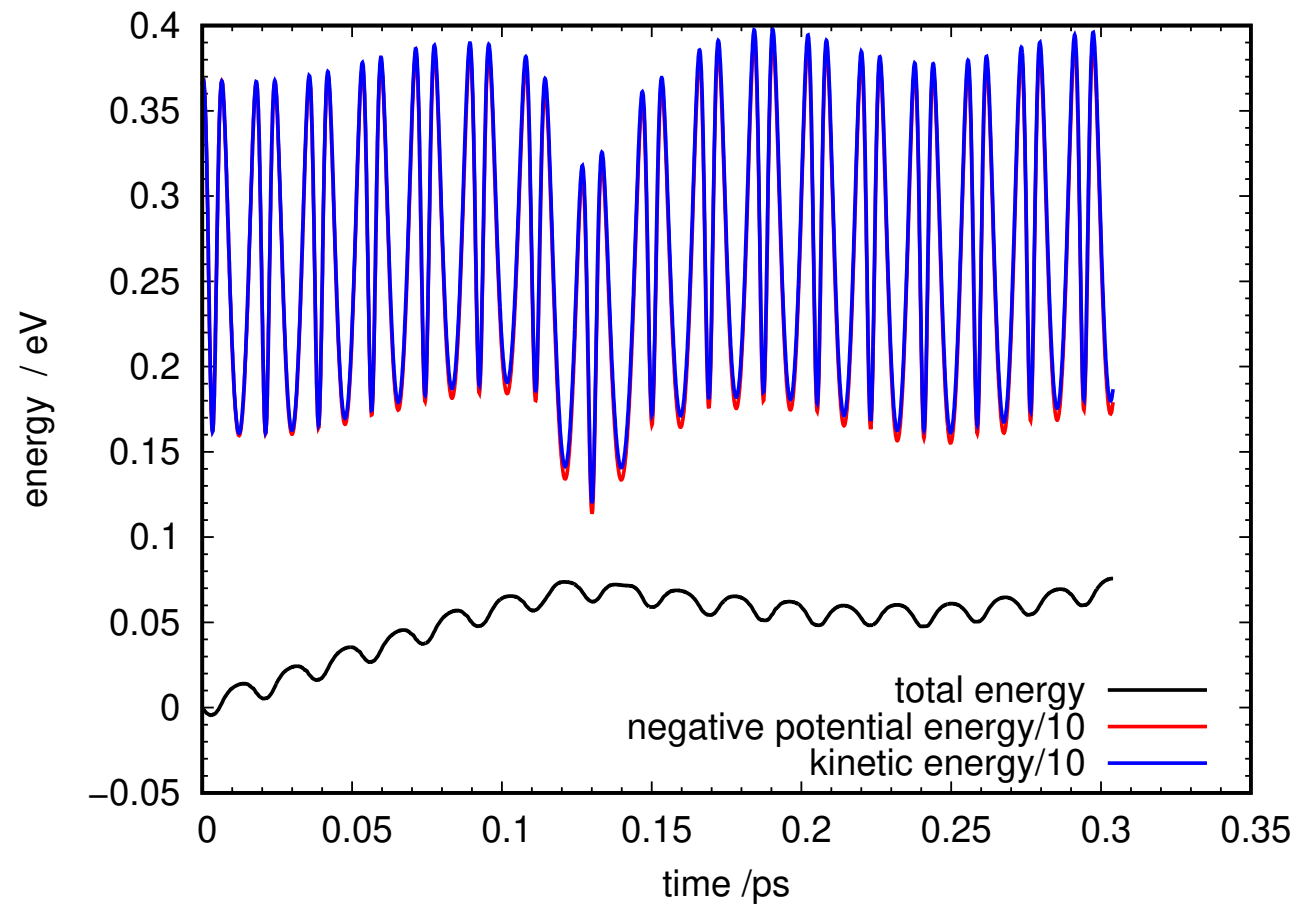

(b) $p(3 \times 3)$-slab with a movable bottom layer.

Fig. 55: Time evolution of the total (black line), kinetic (blue) and negative potential energies (red) of the system for a trajectory of $\mathbf{C O}\left(T_{\mathrm{in}}=1 \mathrm{eV}, E_{\mathrm{vib}}=2 \mathrm{eV}\right)$ approaching an $\mathbf{A u}(111) p(3 \times 3)$ slab with 4 layers at $300 \mathrm{~K}$. The kinetic and potential energies are scaled by a factor of 10.

mentioned above, the bottom layer of the slab was kept fixed to prevent the slab from drifting through the simulation cell. As this was adopted from the VASP calculations it 
may not be a correct treatment for this issue within FHI-aims. Therefore, I removed the constraints from the last layer and gave those atoms a velocity of zero. The outcome of this simulation is shown in Fig. 55b. It reveals a reduction of the energy drift of about $50 \%$ with respect to the trajectory with a fixed last layer as shown in Fig. 55a. So, this setting seems to be promising for doing simulations with highly-vibrationally excited $\mathrm{CO}$ from metal surfaces. However, the reason for the difference in the energy drift of the two different settings is not completely clear but seems to be linked to the treatment of the COM motion by FHI-aims. The latter means that the code corrects for motion of the COM by subtracting translation of the system and correcting the forces [105]. Of course, this correction affects the total energy because the velocities of all atoms are changed and therefore their kinetic energy but not potential energy is affected. However, instead of using the setting with all slab atoms movable I decided to switch off the correction of the forces and used a slab with a rigid last layer to maintain a slab configuration, a setting most similar to VASP. Therefore, I set the clean_forces keyword to . none. and constrain_relaxation keyword to .true. for the atoms in the bottom most layer of the slab, when doing simulations of CO in a high vibrational state scattered from metal surfaces which are presented below.

After the promising results of a relatively small drift of the total energy for the trajectories calculated with FHI-aims, I started simulations for $\mathrm{CO}(\nu=17)$ scattered from $\mathrm{Au}(111)$ and $\mathrm{Ag}(111)$ at $300 \mathrm{~K}$. I used the following setting to control the simulation: The electronelectron interaction was described by the RPBE functional in the spin-unpolarized treatment, as CO is a closed-shell molecule. The electronic occupation was modeled by the Gaussian smearing with a width of $0.2 \mathrm{eV}$ and the IBZ was sampled by a $4 \times 4 \times 1$ $\boldsymbol{k}$-point grid. The simulation time step was set to $0.5 \mathrm{fs}$. The surfaces were modeled by a $p(3 \times 3)$ cell with 4 layers — built up by an optimized lattice constant of $4.20 \AA$ for both metals - and a vacuum distance in $z$-direction of $20 \AA$ was used. To simulate the temperature of the surface the procedure for slab-equilibration was used, as described in 4.1. The CO molecule was set at $z_{\mathrm{cm}}=5.5 \AA$ above the slab. All other parameters to initialize the simulations were determined as described in subsection 4.2.1, using an initial translational energy of $0.58 \mathrm{eV}$ and a vibrational energy of $4.1 \mathrm{eV}$ corresponding to $v=17$. A trajectory was stopped if the molecule was scattered back and its COM-slab distance was $>5.5 \AA$ or after a maximum simulation time of 1 ps.

The outcome of the simulations was analyzed as described in section 4.3, which leads to energy distributions for the different dof presented in the following paragraph. In Fig. 56 the final translational energy distribution of $\mathrm{CO}(v=17 \rightarrow 17)$ scattered from $\mathrm{Au}(111)$ and $\mathrm{Ag}(111)$ for experiment and the computed AIMD simulations are shown, and thus the outcome of the vibrationally inelastic scattering channel is shown. 


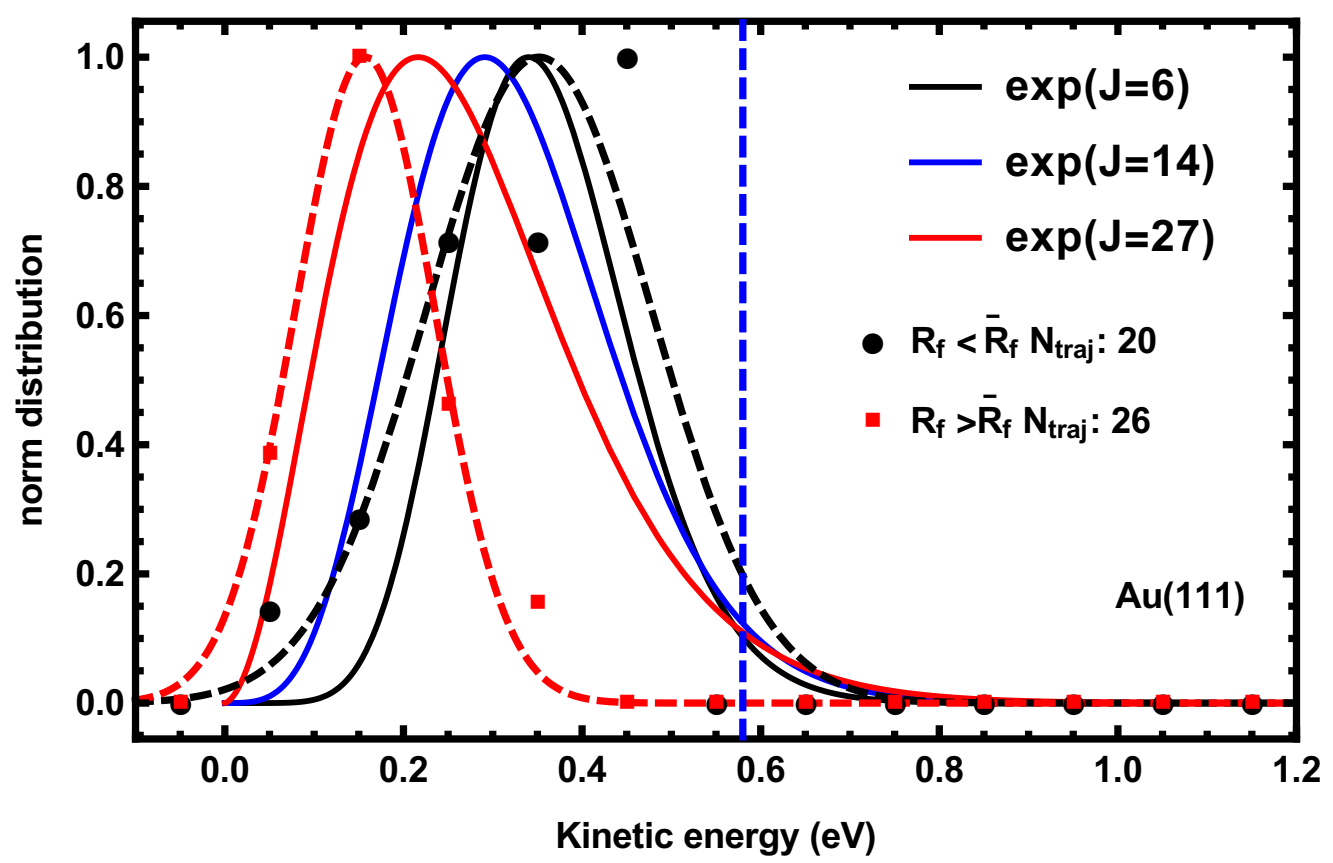

(a) Fits of the normalized (to maximum) final translational energy distribution for $\operatorname{CO}(v=17 \rightarrow 17)$ scattered from Au(111) for different rotational states $J$ for the experiment (solid black, solid red and blue lines) and AIMD (dashed black and dashed red lines) for low and high rotational energies. The AIMD data are represented by black circles and red squares. The vertical blue dotted line represents the incidence energy.

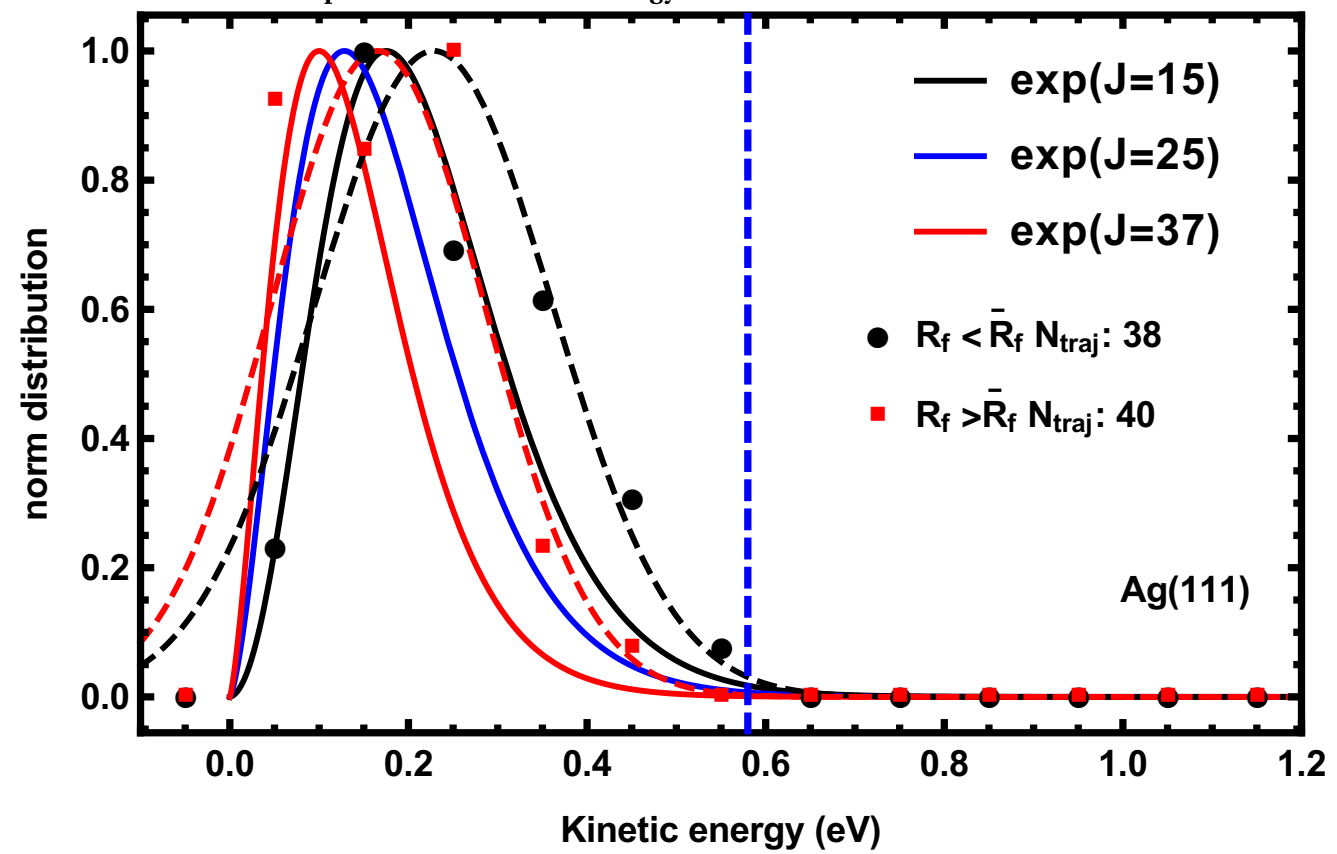

(b) Fits of the normalized (to maximum) final translational energy distribution for $\mathbf{C O}(v=17 \rightarrow 17) \operatorname{scattered~from~} \mathrm{Ag}(111)$ for different rotational states for the experiment (solid black, red and blue lines). The AIMD data are represented by black circles and red squares with fits as dashed black and dashed red lines. The blue dotted lines represents the incidence energy.

Fig. 56: Final translational energy distribution for $\mathbf{C O}(v=17 \rightarrow 17)$ scattered from $\mathbf{A u ( 1 1 1 )}$ (a) and $\operatorname{Ag}(111)$ (b) for the experiment and AIMD simulations done with the RPBE functional.

The fits of the experimental data [42] and the computed AIMD simulations seems to agree qualitatively well for both surfaces, although the number of trajectories to obtain the corresponding fits is small, around 20-40. The translational energy loss is higher 
in case of $\mathrm{Ag}(111)$ than on $\mathrm{Au}(111)$ for both the experiment and simulations. When the molecules have higher rotational energy, they are in a higher rotational state, then their final translational energy is lower, which is also observed in the experiment and simulations.

After the description of the results for the vibrationally inelastic scattering I come to the vibrationally elastic case. Therefore, the final vibrational state distributions for $\mathrm{CO}\left(v_{\text {in }}=17\right)$ scattered from silver (top panel) and gold (bottom panel) (111)-surfaces of the AIMD-simulations and experiment are presented in Fig. 57. In the case of Ag(111) the vibrational state distributions obtained from the AIMD simulations and the experiment show differences. Thus, in the experiment most of the scattered molecules relax into a lower vibrational state than the initial one, whereas in the case of the simulations (black crosses) most of the molecules remain in the initial vibrational state $(v=17)$. Furthermore, the AIMD simulations show a small number of trajectories which show vibrational excitation, which is not observed experimentally.

For the vibrational state distributions obtained for $\mathrm{Au}(111)$ the picture is different. Here, the calculations and experimental results agree very well. Hence the amount of molecules which undergoes vibrational relaxation during the encounter with the surface is similar. Also in this case a small fraction $(<10 \%)$ of the trajectories gain vibrational energy during the encounter with slab.

Therefore, the AIMD simulations and experimental data in the case of $\mathrm{Au}(111)$ are in better agreement than in the case of $\mathrm{Ag}(111)$. Thus, in the first case the vibrational energy redistribution observed in experiment can be described by simulations on a ground state PES; that means the energy transfer between molecule and the surface can be described in an adiabatic picture. This seems only partly to be the case for the vibrational energy transfer between the molecule and the $\mathrm{Ag}(111)$ surface. Here, the simulations show less vibrational relaxation than experimentally observed, i.e. $30 \%$ of the molecules relax into lower vibrational states in former case and about $60 \%$ in the latter case. Therefore, non-adiabatic effects seem to play a larger role in the scattering from the $\mathrm{Ag}(111)$ surface than from the $\mathrm{Au}(111)$ surface.

An explanation for this difference between the two surfaces can be the difference between the work functions of the two metals, which is $5.31 \mathrm{eV}$ for $\mathrm{Au}(111)$ and $4.74 \mathrm{eV}$ for $\operatorname{Ag}(111)$ [162]. The lower work function of $\mathrm{Ag}(111)$ makes it possible to excite electrons on the surface more easily than on the Au(111) surface. Thus, ehp excitation due to molecular vibrational energy is more pronounced in $\operatorname{Ag}(111)$ than in $\mathrm{Au}(111)$ which cannot be described by AIMD simulations on a single PES. For this reason the simulations in case of $\operatorname{Ag}(111)$ describe the experimental result of the final vibrational state distribution better than in case of $\mathrm{Au}(111)$. 


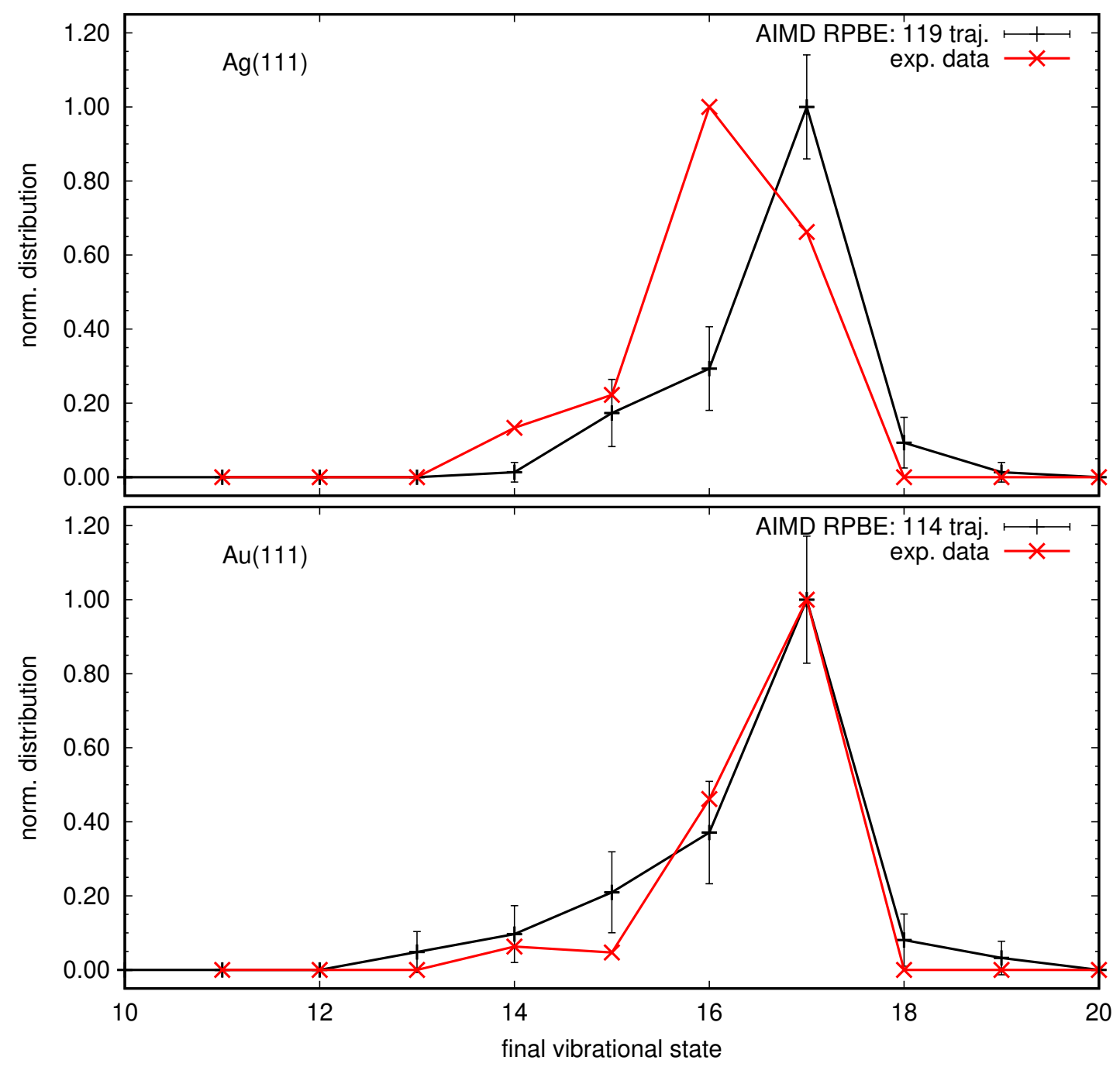

Fig. 57: Normalized (to maximum signal) final population of the vibrational states $v$ is shown for $\operatorname{CO}\left(v_{\text {in }}=17\right)$ scattered from $\mathrm{Ag}(111)$ (top panel) and Au(111) bottom panel. Shown are AIMD simulations (black) and the corresponding experimental data (red). The number denotes the total number of scattered trajectories. 
I looked in more detail into the vibrational states distribution with respect to the initial molecular orientation. For this purpose, I divided the angle $\theta-$ the angle between the $z$-axis and the bond vector $\boldsymbol{r}$ - into three equal sub-groups from 0 to $\pm 60^{\circ}$, from $\pm 60^{\circ}$ to $\pm 120^{\circ}$ and from $\pm 120^{\circ}$ to $\pm 180^{\circ}$, representing $C$ down, CO parallel and $O$ down orientation, respectively. The outcome of this analysis is shown in Fig. 58. It reveals that most of the scattered trajectories which undergo relaxation encounter the slab initially in the parallel molecular orientation. The two other orientations are more pronounced in the initial state $(\nu=17)$, where $C$ down shows more relaxation than $O$ down. This can be observed for silver and gold as well. Therefore, a transfer of vibrational energy in other dofs seems to be unlikely when the molecule approaches with $\mathrm{O}$ oriented to the surface.
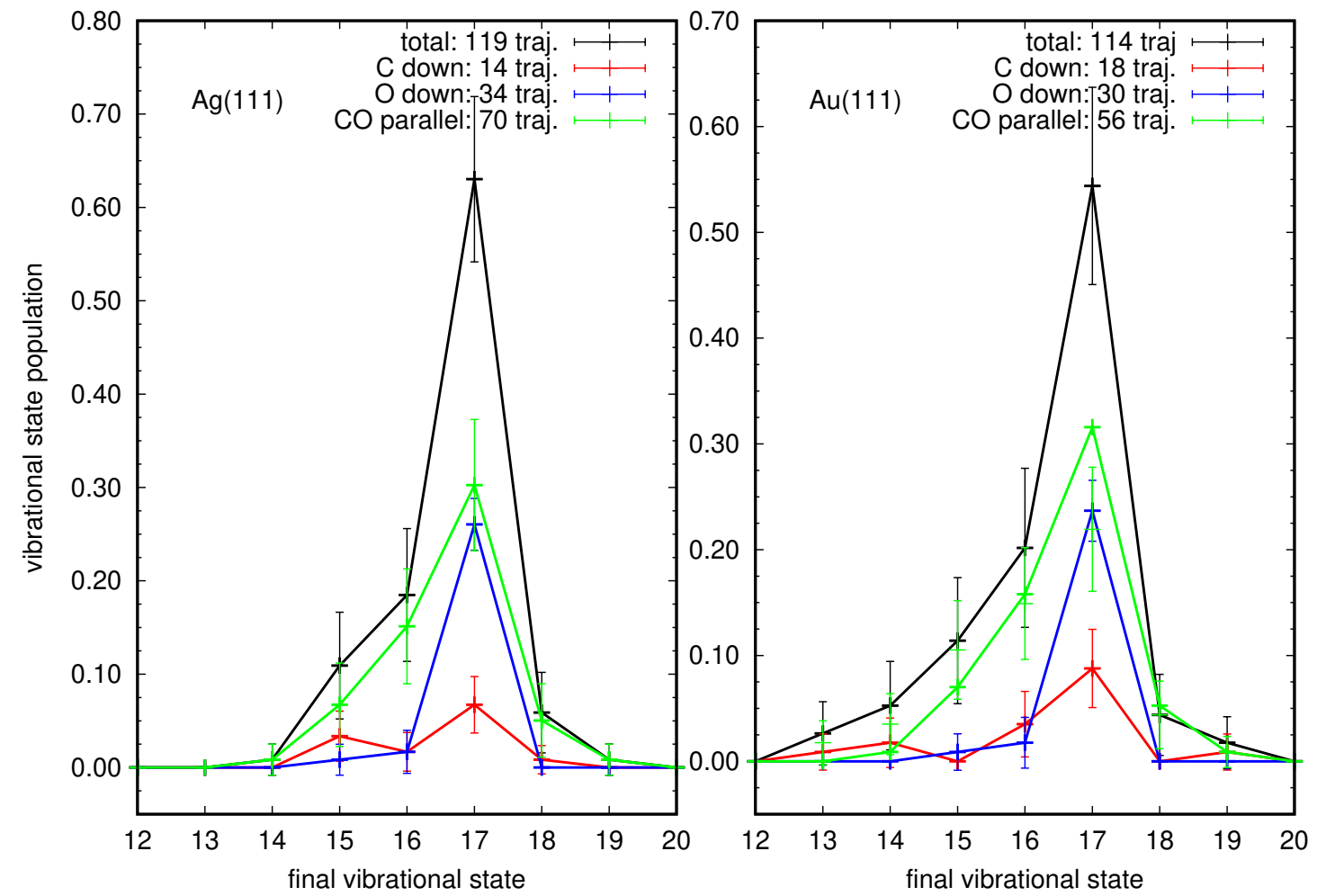

Fig. 58: Final vibrational state distribution (normalized to the total number of trajectories (probability density)) for $\mathbf{C O}\left(v_{\mathrm{in}}=17\right) / \mathbf{A g}(111)$ (left) and $\mathbf{A u}(111)$ (right). The black, red, blue and green lines are representing all trajectories and those which are initial $\mathrm{Cdown}, \mathrm{O}$ down, and $\mathrm{CO}$ parallel oriented, respectively. The numbers are representing the numbers of the scattered trajectories and their fraction of the total number in \%.

I also performed simulations for $\mathrm{CO}$ in $v=22$, thus with a large amount of vibrational energy $\left(E_{\mathrm{vib}, \text { in }}=5.2 \mathrm{eV}\right)$, from $\mathrm{Au}(111)$ and $\mathrm{Ag}(111)$ to see if there is a possibility that the trajectories may show dissociation on the surface, but also to see if there is any difference in their vibrational relaxation or excitation compared to the molecule in $v=17$. Except for the initial vibrational energy the input parameters were the same as 
before, thus a $\mathrm{p}(3 \times 3)$ slab with 4 layers equilibrated at $300 \mathrm{~K}$ and a translation energy of $0.58 \mathrm{eV}$ were used to initialize the simulations.

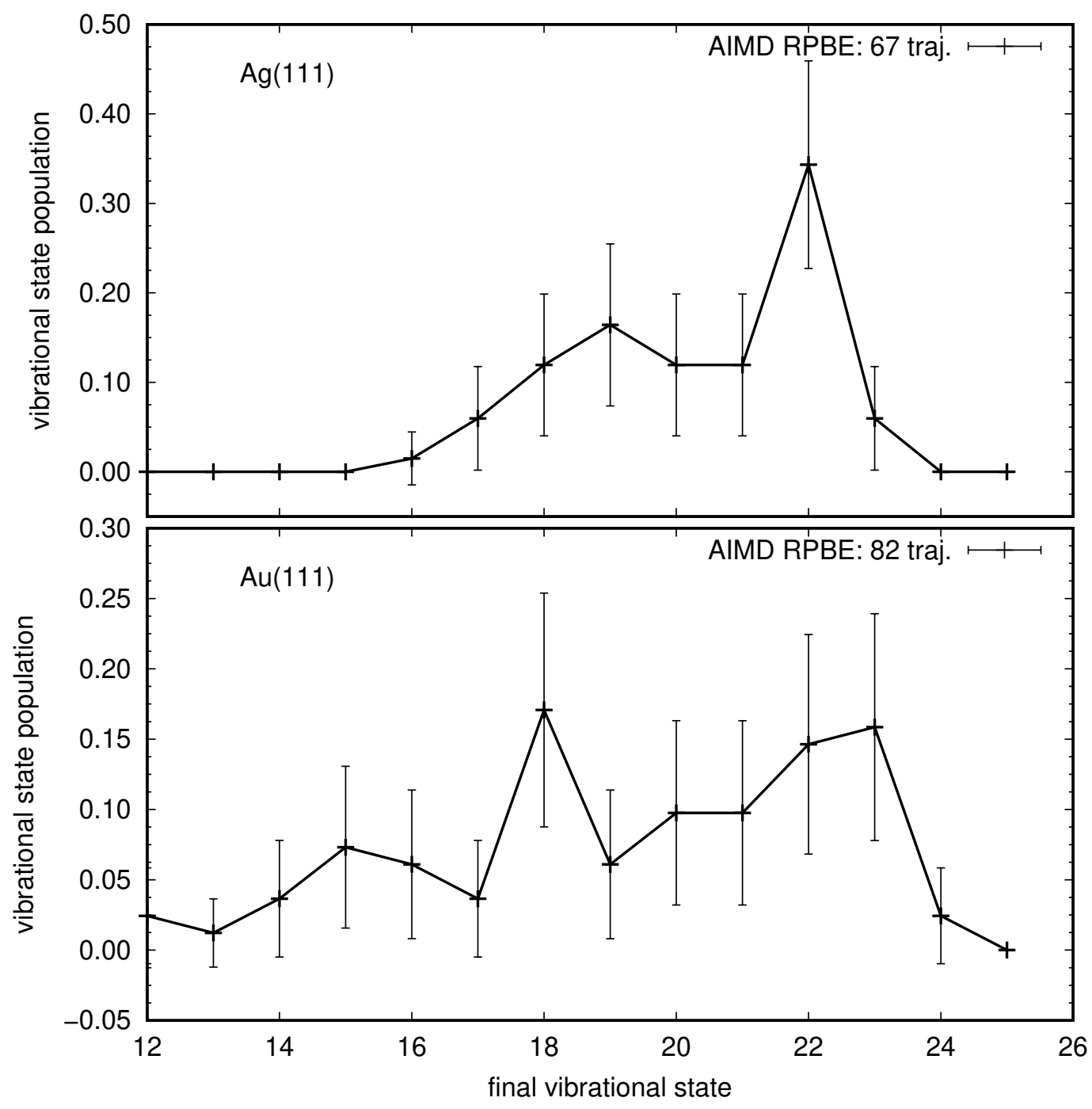

Fig. 59: Final vibrational state distribution (probability density) for $\operatorname{CO}\left(v_{\text {in }}=22\right)$ scattered from $\operatorname{Ag}(111)$ (top panel) and Au(111) (bottom).

The final vibrational state distributions for both surfaces are shown in Fig. 59. For both surfaces, $\mathrm{Ag}(111)$ and $\mathrm{Au}(111)$, the scattered trajectories undergo vibrational relaxation and excitation. The fraction of molecules which remain in the initial $v$-state is above $25 \%$ when CO is scattered from $\mathrm{Ag}(111)$, but only $8 \%$ in case of $\mathrm{Au}(111)$. The vibrational relaxation covers many vibrational states (up to 10 states for $\mathrm{Au}(111)$ ), thus a few trajectories lose more than $2 \mathrm{eV}$ of vibrational energy, whereas the vibrational excitation is only seen in the higher adjacent state with respect to the initial state. However, the results can only give first insights in the final vibrational state distributions as the number of trajectories is very small, and the statistical error is large. This is due to a relatively large 
number of trajectories which was not scattered back from the surface. CO molecules for $v_{\text {in }}=22$ exhibit a higher vibrational relaxation than CO in $v_{\text {in }}=17$ (compare Figs. 57 and 59).

Finally, a look into the bond length of the molecule along all (including ones which where not scattered back) trajectories reveals that its maximum value was $<=1.75 \AA$ in the case of $\operatorname{CO}\left(\nu_{\mathrm{in}}=17\right)$ and was $<=2.05 \AA$ in the case of $\operatorname{CO}\left(\nu_{\mathrm{in}}=22\right)$. Thus, it seems to be unlikely that a large fraction of molecules dissociates during the encounter with the surface. This seems to be reasonable, because the experimentally determined dissociation energy for the molecule in vacuum is $11.45 \mathrm{eV}$ [146] and value for $D_{\mathrm{e}}$ determined here (see section 8.3 ) is $11.579 \mathrm{eV}$ which is around $2 \times$ larger than the initial vibrational energy of the molecule.

\subsubsection{Analysis of the forces}

Here I want to make a remark about the forces computed during the AIMD simulations with FHI-aims. Analyzing the forces reveals that the sum of all forces in the system is in the range of $0.05 \mathrm{eV}^{-1}$. This is inconsistent with the relatively small drift in the total energy, which is under $100 \mathrm{meV}$. The further analysis shows that the deviation of the sum of all forces from zero is mainly due to the forces acting on the COM of the molecule which leads to a slight acceleration of the molecule from the surface.

In Fig. 60 the evolution of the sum of all forces in the system (panel c), the forces acting on CO projected on the bond vector and perpendicular to this vector (panel d), and the sum of all forces acting on the slab atoms (panel e) along a trajectory of $\mathrm{CO}\left(T_{\mathrm{in}}=0.58 \mathrm{eV}, v=17\right)$ scattered from $\mathrm{Ag}(111)$ at $300 \mathrm{~K}$ are shown. Furthermore, the change in total energy of the system, the translational and the rotational energy of the molecule (panel b), and the distance of the $\mathrm{C}$ and $\mathrm{O}$ atom to the surface (panel a) are presented. The components of the total force of the system as well as the projection of the forces on the bond length do not vanish even when the molecule is more than $6 \AA$ away from the surface. The total energy of the system and translational energy of the molecule do not show constant values even when the interaction between molecule and slab should be negligible.

The amplitude of the change for these energies is about $0.025 \mathrm{eV}$ in the part of the trajectory where the molecule is far from the surface (see panel a of Fig. 60) due to the non-vanishing forces on the CO molecule and the total system. The rotational energy averaged over a period is constant far from the surface, thus it is not influenced by the varying forces (see blue line in panel b). The $x, y$-components of the force acting on all slab atoms (see panel e)) are close to zero when the molecule is far away from the 


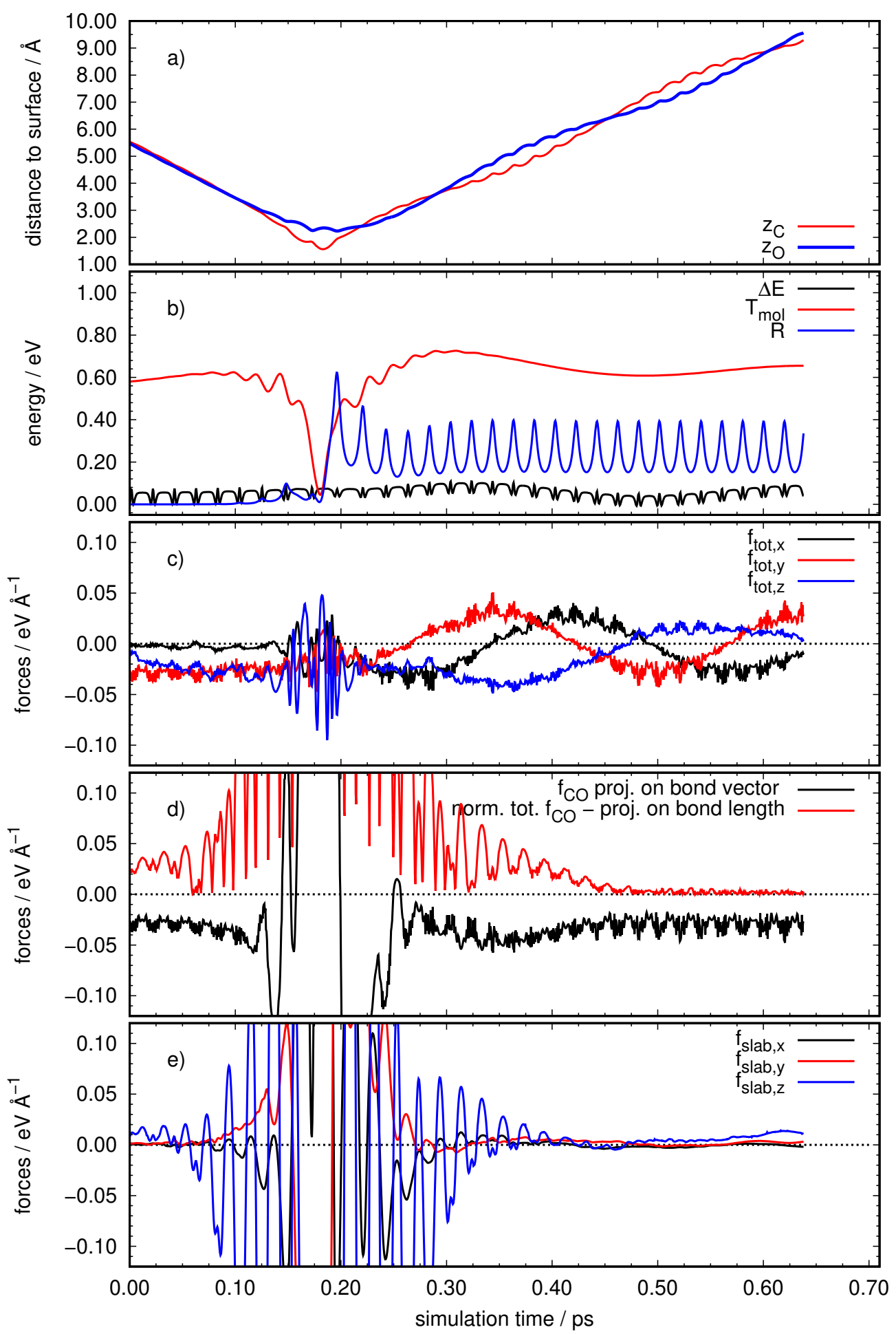

Fig. 60: A selected trajectory for $\mathrm{CO}(v=17) / \mathrm{Ag}(111)$ at $300 \mathrm{~K}$. Shown are the $z$-coordinates of $\mathrm{C}$ and $O$ (panel a), the total energy drift of the system and the translational and rotational energy of the molecule (panel b); the $x, y$ and $z$-components of the force on the total system (panel c) and on all slab atoms (panel e); the projection of the forces acting on $\mathrm{CO}$ along the bond and the norm of the difference between the total forces acting on $\mathrm{CO}$ and this projection (panel d). 
slab, whereas the $z$-component has a value up to $0.02 \mathrm{eV}^{-1}$ when $\mathrm{CO}$ is more distant to the slab. But this clearly does not cause the deviation in the total forces of the system from zero, as seen in panel c). The reason for this deviation is due to the forces acting on CO, especially to the forces acting in the direction of the bond distance. This can be concluded from panel d) where the projection of these forces on the bond vector $r$ (black) and the norm of the difference between the forces acting on $\mathrm{CO}$ and this projection (red) are shown. The latter is nearly zero and the former is about $0.03 \mathrm{eV} \AA^{-1}$ in the case when the molecule is more than $4 \AA$ away from the surface.

However, these deviations from the energy conservation law should not significantly affect the energy distributions shown in Fig. 56, as the binning used to produce the distributions is $0.05 \AA$.

\section{5 $\mathrm{NO}(v=16)$ from $\operatorname{Au}(111)$ and $\operatorname{Ag}(111)$}

For the simulations of scattering of $\mathrm{NO}(\nu=16)$ I used a similar setting as for the simulations of CO. So, the light-setting for the species basis sets was used. In contrast to $\mathrm{CO}$, NO is an open-shell system and so the spin-polarized GGA including RPBE was employed in the calculations. Therefore, the initial spin moments for $\mathrm{N}$ and $\mathrm{O}$ were set to 3 and 2, respectively. Since the spin-polarized treatment strongly increases the computational effort compared to the unpolarized treatment used for $\mathrm{CO}$, it was necessary to reduce the system size so that the calculation could be done in a bearable manner. For this reason the slab size was reduced from 4 to 2 layers. The atoms of the bottom layer were kept fixed at their equilibrium positions during the simulations with $\mathrm{NO}$ as well as in the equilibration-run. The initial translational energy of NO was set to $0.58 \mathrm{eV}$ and the vibrational energy of $v=16$ was $2.960 \mathrm{eV}$. The latter was obtained by fitting the Morse function to DFT data for different molecular bond distances, for the molecule without slab. The fit parameters are $r_{\mathrm{eq}}=1.140 \AA, \alpha_{\mathrm{M}}=2.736 \AA^{-1}$ and $D_{\mathrm{e}}=7.316 \mathrm{eV}$.

In Fig. 61 the final vibrational state distributions of $\mathrm{NO}\left(v_{\text {in }}=16\right)$ of the scattered trajectories from $\mathrm{Au}(111)$ and $\mathrm{Ag}(111)$ as well as the experimental data for $\mathrm{Au}(111)$ are shown.

The distributions obtained from the AIMD simulations show vibrational relaxation and in the case of $\mathrm{Ag}(111)$ vibrational excitation. The fraction of trajectories which remains in the initial vibrational state $\left(v_{\text {in }}=16\right)$ is larger in case of $\operatorname{Ag}(111)$ than $\operatorname{Au}(111)$. But, due to a broader distribution in the case of $\mathrm{Ag}(111)$ it is difficult to make a clear statement as the error bars are very large in these cases. In the experiment only $2 \%$ of the molecules remain in their initial $v$-state, whereas in the case of the adiabatic AIMD simulations it is $15 \%$. Furthermore, in comparison to the experimental data 


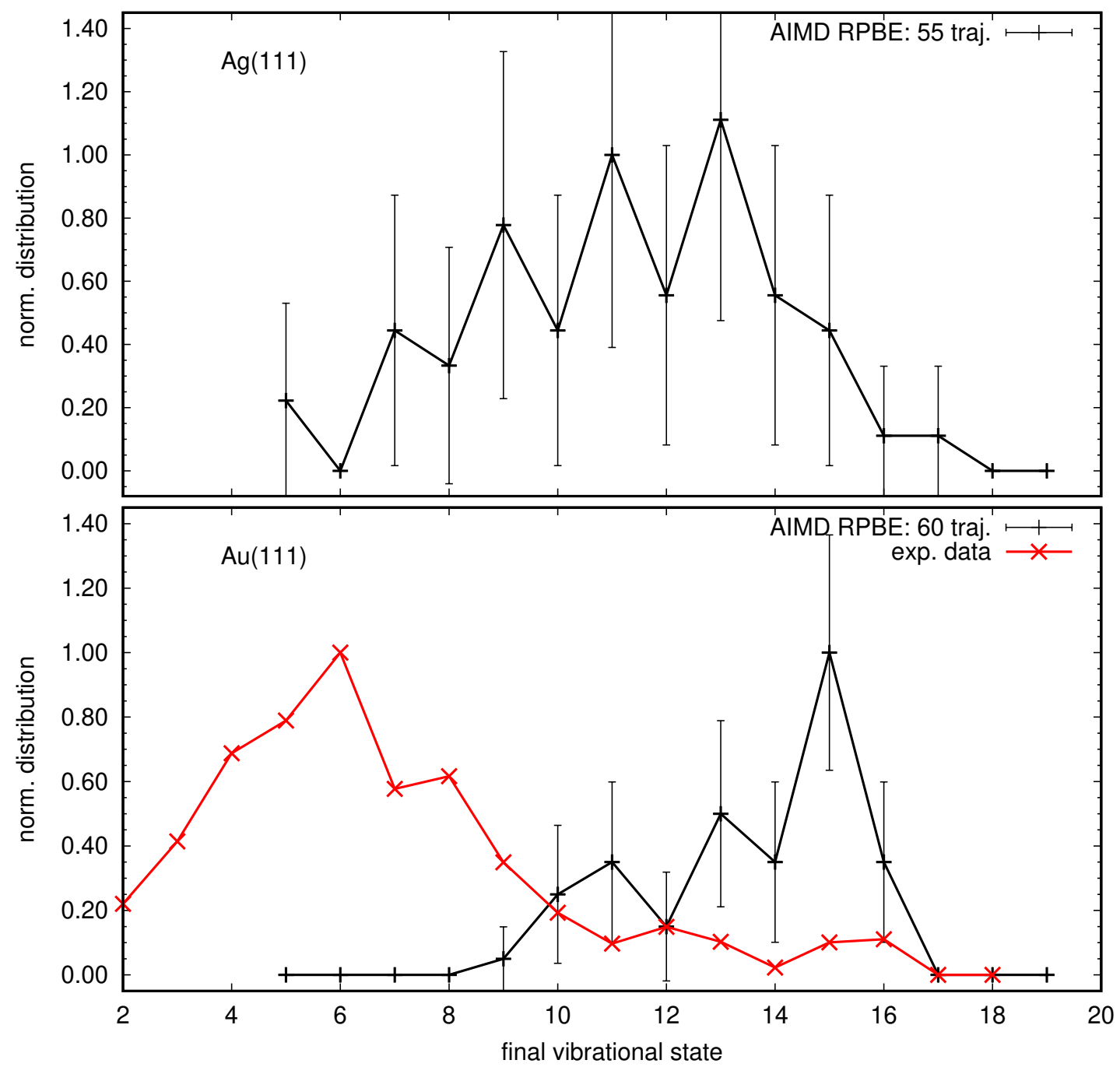

Fig. 61: Normalized (to maximum signal) final vibrational state distribution for $\mathrm{NO}\left(\nu_{\text {in }}=16\right)$ scattered from Ag(111) (top) and Au(111) (bottom ). Results of the AIMD simulations and experimental data (in case of $\mathrm{Au}(111)$ ) are shown in black and red, respectively. 
the distribution obtained from the simulations clearly exhibits less (multi) vibrational relaxation so that in the simulations the molecule loses only up to 6 vibrational quanta, whereas the experiment reveals a population of quantum states up to 14 quanta lower than the initial state. Therefore, these simulations on a single PES can clearly not explain the experimental results, and non-adiabatic effects seem to play a large role in the scattering of highly-vibrationally excited NO from $\mathrm{Au}(111)$.

Although the number of trajectories is relatively small for both surfaces, the vibrational relaxation seems to be much stronger than for CO in a similar initial $v$-state scattered from these surfaces (compare Fig. 61 to Fig. 57).

\subsection{Conclusions}

AIMD simulations are a powerful tool to investigate the energy transfer when a molecule is scattered from a metal(111) surface. Especially, we can make a statement about the importance of non-adiabatic effects, like ehp excitation, in this collision by comparing the results of the scattering experiments with the AIMD (adiabatic) simulations.

The AIMD simulations of CO scattering from different metal (111) surfaces where $\mathrm{CO}$ is in low vibrational states clearly show a nearly quantitative agreement with the experiment. So simulations carried out on the ground state PES can describe the experimental observations for $\mathrm{CO}$ in low vibrational states. Therefore, we can conclude that the scattering of $\mathrm{CO}$ in a low vibrational state is not affected by non-adiabatic effects and the energy transfer between molecular and surface dofs can be described by purely mechanical considerations. Since no vibrational relaxation or excitation is observed it seems unlikely that energy is transferred between the molecular vibration and electronic dofs of the surface. This also supported the adiabatic picture of the molecule-surface interaction in the case of a low vibrational excited molecule.

To achieve reliable results of AIMD simulations with highly vibrationally excited CO a change from VASP to FHI-aims was necessary, since in VASP the drift in total energy of the AIMD with highly vibrationally excited CO approaching the Au(111) slab is too large. Tests with the CO molecule in vacuum reveals that this large energy drift is due to convergence problems, when the $\mathrm{CO}$ bond distance is in a certain range. This is due to competing spin states. Trying different parameters did not solve the problem. Calculations done with FHI-aims do not show such problems.

The performed AIMD simulations of scattering of $\mathrm{CO}\left(v_{\mathrm{in}}=17\right)$ from $\mathrm{Au}(111)$ and $\mathrm{Ag}(111)$ give translational energy distributions for the vibrationally inelastic channel, which are in qualitative agreement with those obtained in experiment. Therefore, we can conclude that the energy transfer in this case between molecule and surface is not influenced 
by non-adiabatic effects, like ehp excitation. Since the latter effects seem to play a dominant role in the redistribution of vibrational energy, the result would support this assumption.

When we look at the final vibrational state distributions, we see that in the case of $\mathrm{Au}(111)$ experimental and simulated results agree nearly quantitatively, whereas in the case of $\mathrm{Ag}(111)$ both distributions show more deviations. Consequently at the gold surface non-adiabatic effects, like ehp excitation, seem to play a negligible role in the energy transfer with CO. In the case of $\mathrm{Ag}(111)$ the influence of non-adiabatic effects seems to be stronger in the energy transfer when the molecule is scattered from the surface. The differences in this behavior may be due to the work functions of the two surfaces. Since the work function is higher for $\mathrm{Au}(111)(5.31 \mathrm{eV})$ than for $\mathrm{Ag}(111)(4.74 \mathrm{eV})$ it is easier to create a negative ion of a molecule at the silver surface.

Comparing the results of the final vibrational state distributions for $\mathrm{NO}\left(\nu_{\text {in }}=16\right)$, adiabatic simulations and experiments differ strongly. Thus, here the interactions during the collision of projectile and surface seem to be dominantly governed by non-adiabatic effects. A stronger role of these effects (an electron transfer) can be expected more prominently in the case of NO than in the case of $\mathrm{CO}$, because the former molecule has a lower electron affinity $(0.026 \mathrm{eV}$ [162]) than the latter (1.326 eV [163]). Moreover, the VEBE, i.e. the energy difference between the anionic and neutral ground state PES of the molecule at a certain bond length, is more positive in the case of NO than for CO. Thus, the highly vibrationally excited $\mathrm{NO}^{-}$is more stable than the neutral NO. This means that for NO the formation of the anionic species is more likely than for CO. For the molecule at a metal surface this is especially the case as the anion is stabilized by the image charge stabilization.

From the work function of the surface and the VEBE of the molecule, corrected for the image charge stabilization, we can define a parameter to describe the likelihood of an electron transfer from the surface to the molecule and thus the occurrence of non-adiabatic effects in the molecule scattering from a metal surface.

This is seen in Fig. 62, where the vibrational survival probability vs. the difference between the VEBE of the molecule and the work function $\Phi$ of the metal surface for the systems investigated here is shown. We can see that for the systems where the probability for an electron transfer is low AIMD simulations and experimental results agree well, whereas when the probability for a transfer increases both results show stronger deviations. Since the occurrence of an electron transfer in the system is clearly a non-adiabatic effect, and such effects are more likely in the right-hand side of the plot, the deviations between simulations and experiment can be attributed to them. The case of $\mathrm{NO}(v=16) / \mathrm{Au}(111)$ does not seem to fit in this picture. Here the difference 


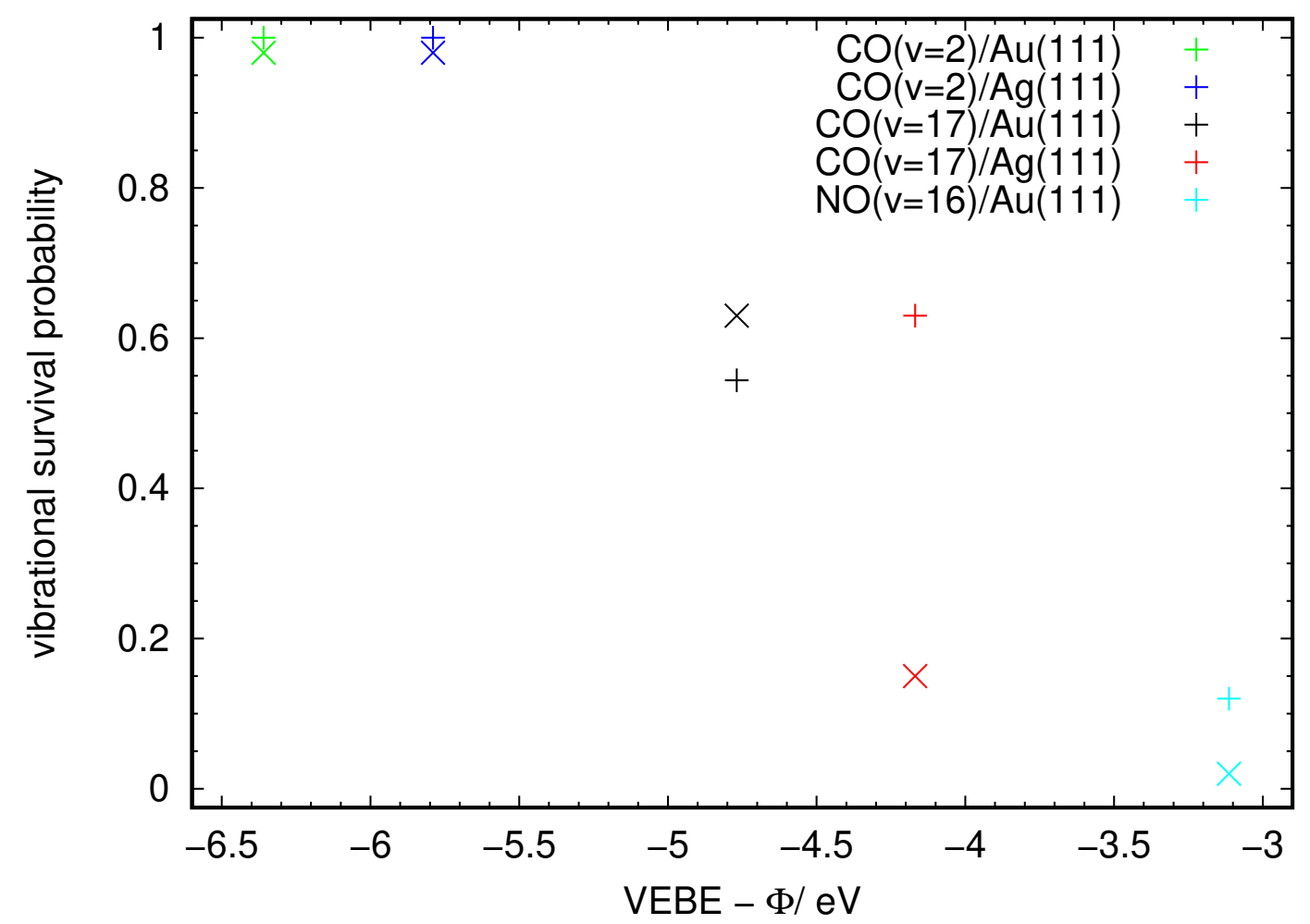

Fig. 62: Vibrational survival probability vs. the difference between the VEBE of the molecule and the work function $\Phi$ of the surface for the here investigated systems, which are indicated by labels. The diagonal crosses represent the experimental data and the crosses represent the results of the AIMD simulations.

between simulations and experiment seem to be smaller than one could expect from the trend of the other systems. However, the role of non-adiabatic effects seems to get important between a VEBE $-\Phi$ of -4.8 to $-4.1 \mathrm{eV}$, and therefore AIMD simulations for a molecule surface system in this range, e.g. $\mathrm{CO}(v=11) / \operatorname{Ag}(111)$ or $\mathrm{NO}(v=3) / \mathrm{Au}(111)$, should be investigated further. 


\section{Transition dipole moments of carbon monoxide on different surfaces}

\subsection{Introduction}

In this section I cover the results of the calculations of the transition dipole moments of CO on different surfaces. The transition dipole moment of the molecule is a quantity which is important to calculate with ab-initio methods, as it can be determined experimentally via spectroscopic methods. Furthermore, the dipole moment gives insights into the electric properties of the molecule at the surface. Thus, differences in the transition dipole moments (or the electric properties) for $\mathrm{CO}$ at $\mathrm{Au}(111), \mathrm{Ag}(111)$ and Ag-covered $\mathrm{Au}(111)$ surfaces can explain the experimentally observed differences in the translational energy distributions of scattered molecules. The procedure to determine the transition dipole moment of CO on the different surfaces is described in section 4.5. First I will describe the calculations for CO on Au(111) for VASP and FHI-aims and compare the results for the two codes. Then I will describe the results for CO on Ag-covered $\mathrm{Au}(111)$ and on $\mathrm{Ag}(111)$ surfaces.

\section{$9.2 \mathrm{CO}$ on $\mathrm{Au}(111)$}

I started the investigation with the geometry optimization of $\mathrm{CO}$ on the $\mathrm{Au}(111)$ surface. The necessary calculations done with FHI-aims were described by the following parameters: The XC were described by RPBE within the spin-unpolarized approach. Further vdW-corrections (TKATCHENKO and SCHEFFLER [152]) were taken into account. The electronic occupation was described by a Gaussian function with a width of $0.2 \mathrm{eV}$. The reciprocal space was sampled via a $4 \times 4 \times 1 \boldsymbol{k}$-point grid which was generated with the MONKHORST scheme. The $s c f$-cycle was stopped when the change in the total force and in the sum of the eigenvalues were smaller than $10^{-4} \mathrm{eV} \AA^{-1}$ and $10^{-4} \mathrm{eV}$, respectively; and the geometry optimization was stopped when the force on every atom was $<0.01 \mathrm{eV}^{-1}$. The pre-optimized structures obtained with light-settings for the basis sets were used to find the energy minimum structures with the larger basis sets of the 
tight-settings for the corresponding species. A $\mathrm{p}(2 \times 2)$ and a $\mathrm{p}(3 \times 3)$ slab with 4 layers (described by $a=4.20 \AA$ ) were used to model a CO coverage of $0.25 \mathrm{ML}$ and $0.11 \mathrm{ML}$, respectively. The width of the vacuum layer in the simulation cell was $20 \AA$. The atoms of the three uppermost layers were allowed to move during the optimization, whereas the atoms of the bottom layer were kept fixed at their equilibrium positions.

For the calculations with VASP the following input parameters were used: a $4 \times 4 \times$ $1 \boldsymbol{k}$-point grid and the RPBE-functional with vdW-corrections by TCHATCHENKOSCHEFFLER. The electronic band occupation was described by a first-order MP function with a $\sigma$ of $0.2 \mathrm{eV}$. As before, the PAW-approach for the plane waves with a cut-off energy of $400 \mathrm{eV}$ was used. The geometry optimizations were stopped when the forces acting on all atoms were $<0.01 \mathrm{eV} \AA^{-1}$. A CO coverage of $0.25 \mathrm{ML}$ was modeled by $\mathrm{p}(2 \times 2)$ slab with 4 layers $(a=4.20 \AA$ ) and one CO molecule on top of it; the width of the vacuum layer was set to $20 \AA$. The atoms of the three uppermost layers of the slab were allowed to move during the structural optimization.

The results of geometry optimizations are presented in Tab. 7. There are only slight differences between the different codes and CO coverage with respect to the structural parameters, thus the $\mathrm{CO}$ bond distance as well as the distance between the $\mathrm{C}$ and the nearest surface atom vary only by about $0.01 \AA$, and CO sits on top site for each case. But, the partial charges on $\mathrm{C}$ and $\mathrm{O}$ depend strongly on the charge analysis program (see sections 3.4 and 4.5). In the case of FHI-aims the charges on $\mathrm{C}$ and $\mathrm{O}$ are about $0.26 e^{-}$and $-0.031 e^{-}$, respectively, whereas in the case of VASP the charges are $1.043 e^{-}$ $(\mathrm{C})$ and $-1.048 e^{-}(\mathrm{O})$. Thus, in the latter case the charges show larger absolute values than in the former case. Furthermore, the total charge on CO is positive in the case of FHI-aims $\left(0.230 e^{-}\right)$and in the case of VASP it is slightly negative $\left(-0.005 e^{-}\right)$.

Tab. 7: Structural parameters of the geometry optimizations for $\mathrm{CO}$ on $\mathrm{Au}(111)$, calculated with FHI-aims and VASP. A CO coverage of $0.25 \mathrm{ML}$ was used, if not otherwise stated. Shown are: the CO bond distance $d_{\mathrm{CO}}$; the distance between $\mathrm{C}$ and the nearest slab atom $d_{\mathrm{C}-\mathrm{M}}$; the angle $\theta$ between $\mathrm{CO}$ bond axis and the surface normal and the partial charges $q_{\mathrm{C}}$ and $q_{\mathrm{O}}$ on $\mathrm{C}$ and $\mathrm{O}$; and the Morse parameters $r_{\mathbf{e q}}, \alpha_{\mathbf{M}}$, and $D_{\mathbf{e}}$.

\begin{tabular}{ccccccccc}
\hline DFT-Code & $d_{\mathrm{CO}} / \AA$ & $d_{\mathrm{C}-\mathrm{M}} / \AA$ & $\theta /{ }^{\circ}$ & $q_{\mathrm{C}} / e^{-}$ & $q_{\mathrm{O}} / e^{-}$ & $r_{\mathrm{eq}} / \AA$ & $\alpha_{\mathrm{M}} / \AA^{-1}$ & $D_{\mathrm{e}} / \mathrm{eV}$ \\
\hline FHI-aims $^{\mathrm{a}}$ & 1.15 & 2.05 & 0.6 & 0.270 & -0.031 & 1.152 & 2.389 & 8.718 \\
FHI-aims & 1.14 & 2.04 & 0.7 & 0.257 & -0.035 & 1.153 & 2.344 & 9.077 \\
VASP & 1.16 & 2.04 & 0.1 & 1.043 & -1.048 & 1.155 & 2.366 & 9.314 \\
\hline
\end{tabular}

${ }^{\mathrm{a} C O}$ coverage: $0.11 \mathrm{ML}$

After I found the minimum energy structures, I used them to calculate the energies and charges on all atoms of the system for different CO bond distances (1.05-1.30 $\AA$ ), in order to determine the change of the dipole moment per surface area (polarization) of the 
system as function of the bond distance and the parameters of a corresponding Morse fit. With the parameters of the Morse fits (see Tab. 7) to describe the wave functions and the functions for the dipole moment operator, I calculated the dipole matrix elements of $\mathrm{CO}$ on $\mathrm{Au}(111)$ and for the molecule in vacuum with Eq. (68). The calculations with the molecule were done with the input parameters, as described above, in a simulation cell with the same dimensions as a p $(3 \times 3)$ slab. Additionally, I did calculations with FHIaims for $\mathrm{CO}$ at different $z_{\mathrm{cm}}$ positions to investigate how the transition dipole moment is affected by the metal slab.
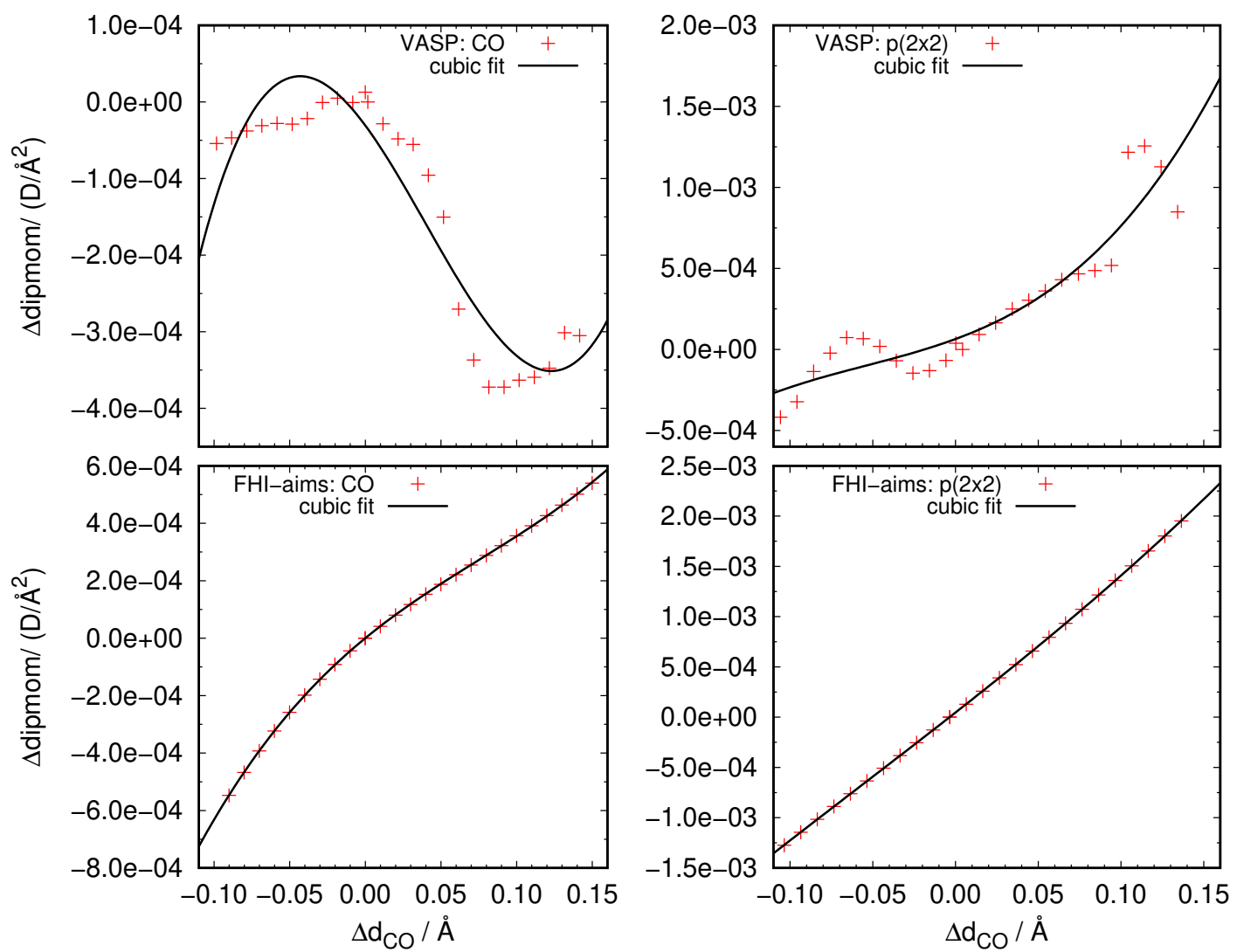

Fig. 63: Change in the dipole moment per area vs. the $\mathrm{CO}$ bond distance with respect to the equilibrium distance for the $\mathrm{CO}$ in vacuum (left) and at the $\mathrm{p}(2 \times 2)$ slab (right). The top and bottom panels show results obtained with VASP and FHI-aims, respectively. The data are shown in red, and the cubic fit is shown as black line.

In Fig. 63 the change in the dipole moment per area projected onto the direction of the $\mathrm{CO}$ bond as function of the CO bond distance is represented for VASP (top panel), FHI-aims (bottom panel), for CO in vacuum and at the $\mathrm{p}(2 \times 2)$ slab with 4 layers. In the case of VASP, both systems show a non-linear behavior for the change in polarization, so that a cubic polynomial does not fit the data well, this is in particular true for the data for the molecule at surface. In contrast the behavior of the polarization in the case of FHI-aims is different, thus the data are fitted very well. Here, also a linear fit would work. 
The amplitudes of the change in polarization show higher values for the molecule at the surface than for the molecule in vacuum, which is seen for both codes. The parameters of the cubic fits, which were used as expression for the dipole moment operator, for all different configurations are shown in Tab. 8.

Tab. 8: Fit parameters of the function given in Eq. (72) to describe the transition dipole moment operator. For $\mathrm{CO}$ and $\mathrm{CO}$ at $\mathrm{Au}(111)$ for different $\mathrm{CO}$ COM-distances to the surface for FHI-aims and VASP. The CO coverage was $0.11 \mathrm{ML}$, where not otherwise stated.

\begin{tabular}{lccccc|cc}
\hline & \multicolumn{9}{c}{ FHI-aims } & \multicolumn{2}{c}{ VASP } \\
$z_{\mathrm{cm}} / \AA$ & 2.72 & $2.78^{\mathrm{a}}$ & 6.00 & 8.00 & CO in vacuum & $2.74^{\mathrm{a}}$ & CO in vacuum \\
\hline$a / 10^{-3} \mathrm{D}^{-5}$ & 11.22 & 24.65 & 28.05 & 26.96 & 61.19 & 121.9 & 168.5 \\
$b / 10^{-3} \mathrm{D}^{-4}$ & 3.89 & 4.43 & -5.57 & -5.82 & -13.87 & 20.01 & -20.15 \\
$c / 10^{-3} \mathrm{D}^{-3}$ & 6.44 & 12.93 & 2.85 & 2.89 & 4.32 & 3.76 & 2.68 \\
$d / 10^{-5} \AA^{-2}$ & 1.48 & 4.65 & 0.50 & 0.24 & 0.02 & 6.44 & 3.08 \\
\hline $\mathrm{a}: 0.25 \mathrm{ML}$ & & & & & \\
\hline
\end{tabular}

The results of the dipole matrix elements for all different configurations and codes are summarized in Tab. 9. The transition dipole moment for the molecule in vacuum in the case of a $0 \rightarrow 1$ transition is smaller than when the molecule is close to an $\mathrm{Au}(111)$ surface. The effect of the cell size, which leads to a higher molecular coverage, is small. The moment of an overtone $(0 \rightarrow 2)$ transition is negative for all cases and is larger for free $\mathrm{CO}$. The diagonal matrix elements are the same represent the expectation values of the dipole moment. These values increase when the quantum number goes up, whereas they decrease when the molecule-slab distance is increased and the smallest value occurs for the molecule in vacuum. This value is much smaller than the value of $0.109 \mathrm{D}$ obtained by fitting experimental data and ab intio calculations [164]. Furthermore, calculations for $\mathrm{CO}$ in vacuum were carried out without periodic boundary conditions. Such it is possible to get the electric dipole moment from the FHI-aims output. For the relaxed molecule the dipole moment projected on the CO bond vector is $0.183 \mathrm{D}$, which is about $10^{4} \times$ higher than its expectation value $(0 \rightarrow 0)$ calculated via the method used here, which is $7.9 \times 10^{-5} \mathrm{D}$. But value from the FHI-aims output is similar to the experimentally determined value mentioned above. In the case of VASP the values calculated for the molecule always have a negative sign compared to $\mathrm{Au}(111)$, hence the direction of the (transition) dipole moment changes when the molecule is adsorbed at the surface. The molecular values for the dipole moment in the ground state, i.e. $n$ and $m$ equals 0 , and the transition dipole moment from $\langle 0|\rightarrow| 1\rangle$ are about $20 \times$ smaller than the experimentally derived values for the molecule of $0.107 \mathrm{D}$ [164]. This is presumably an indication that the basic assumption for the wave functions is not 
justified, hence the wave function of the molecule at the surface differ strongly from that of the molecule in vacuum. For the calculation done with VASP the absolute values of matrix elements for $\mathrm{CO} / \mathrm{Au}(111)$ are similar to the value of the molecule. For $\mathrm{CO}$ at $\mathrm{Au}(111)$ the matrix elements calculated with the output of both codes show similar values for the expectation value of the dipole moment but differ strongly for the transition dipole moments. The single transitions show a larger moment than those of the two-folded one which is symmetry-forbidden; that means the symmetry of the wave function does not change. The absolute values of the single transitions increase with the number $n$. Thus the $1 \rightarrow 2$ is larger than the $0 \rightarrow 1$ transition. The ratio between these transitions is close to $\sqrt{2}$ for all cases. This reflects the harmonic behavior of the Morse potential in this low vibrational states, since the ratio between the $1 \rightarrow 2$ and the $0 \rightarrow 1$ transition is $\sqrt{2}$ for the harmonic oscillator. This can be shown by calculating the corresponding ratio for the harmonic case.

The vibrational lifetime of $\mathrm{CO}(v=1)$ at $\mathrm{Au}(111)$ is according to KUMAR et. al. about 49 ps [165]. From it we can calculated the transition dipole moment from $0 \rightarrow 1$ with the vibrational frequency of $\mathrm{CO}$ at $\mathrm{Au}(111)\left(2131 \mathrm{~cm}^{-1}\right)$ we obtain a value of $2593 \mathrm{D}$. This is 5-6 magnitudes larger than the values calculated here. Since, the differences of the transition dipole moment between the $\mathrm{CO}$ in vacuum and $\mathrm{CO}$ at the surface is larger in the case of FHI-aims these calculations seems to agree slightly better with experiment than the calculations done with VASP.

Tab. 9: Matrix elements $\langle n|\widehat{\mu}| m\rangle$ of the dipole moment in $\times 10^{-3} \mathrm{D}$ for $\mathrm{CO}$ in vacuum and at an $\mathrm{Au}(111)$ surface for FHI-aims and VASP. The numbers in the head of the table represent the COMdistance of the molecule to the surface $\left(z_{\mathrm{cm}}\right)$ in $\AA$ and the CO coverage is $0.11 \mathrm{ML}$ if not otherwise stated.

\begin{tabular}{ccccccc|cc}
\hline \multicolumn{1}{c}{ FHI-aims } \\
$n$ & $m$ & 2.72 & $2.78^{\mathrm{a}}$ & 6.00 & 8.00 & CO in vacuum & $2.74^{\mathrm{a}}$ & CO in vacuum \\
\hline 0 & 0 & 3.347 & 3.332 & 0.756 & 0.540 & 0.079 & 3.293 & -4.343 \\
0 & 1 & 15.890 & 14.108 & 6.803 & 6.868 & 4.769 & 4.818 & -5.512 \\
0 & 2 & -0.438 & -0.549 & -0.934 & -0.968 & -0.910 & -0.961 & -1.418 \\
1 & 1 & 8.150 & 7.267 & 1.611 & 1.382 & 0.314 & 6.298 & -8.213 \\
1 & 2 & 22.923 & 20.284 & 9.773 & 9.837 & 6.850 & 8.059 & -6.481 \\
\hline${ }^{\mathrm{a}}: 0.25 \mathrm{ML}$ & & & & & \\
\hline
\end{tabular}

\subsection{CO on Ag(111) and Ag-covered Au(111)}

As stated in 4.5 I started with the calculation of the optimized structure of CO on the considered surfaces using FHI-aims with a similar input setting as described in the 
previous section. In cases of the metallic (111)-surfaces I used a $\mathrm{p}(3 \times 3)$ cell with 4 layers. The atoms of the bottom layer were kept fixed during the relaxation. To model the Ag-covered surfaces I used slabs with the same procedure as described in chapter 5. The optimized structures show that CO sits on top of a surface atom in each investigated case. This is in agreement with the observation from the calculations of the interaction energies of $\mathrm{CO}$ and those surfaces in section 7.3. The optimized structures do not show strong differences. This can be concluded from the parameters presented in Tab. 10. When the Au atoms are replaced by Ag the distance between molecule and nearest metal atom increases about $0.25 \AA$, and the molecule is tilted about $20^{\circ}$ to the slab in the case of silver. In its minimum position the molecule loses up to $0.24 e^{-}$of its electronic charge to the slab, when the distance to the surface is the smallest. In the case of $\mathrm{Au}(111)$, the COM distance of the molecule to the surface as obtained from minimum energy structure was used to estimate the vibrational lifetime of $\operatorname{CO}(\nu=1)$ at $\operatorname{Au}(111)$ using the model by LIEBSCH [166]. The lifetime was calculated to $0.1 \mathrm{~ns}$ as published by SHIRHATTI et al. [167].

Tab. 10: Structural parameters for the geometry optimizations for CO on different $\mathbf{p}(3 \times 3)$ slabs with 4 layers. Shown are the $\mathrm{CO}$ bond distance $d_{\mathrm{CO}}$, the distance between $\mathrm{C}$ and the nearest slab atom $d_{\mathrm{C}-\mathrm{M}}$, the angle $\theta$ between $\mathrm{CO}$ bond axis and the surface normal, and the partial charges on $\mathrm{C}\left(q_{\mathrm{C}}\right)$ and $\mathrm{O}\left(q_{0}\right)$.

\begin{tabular}{cccccc}
\hline System & 4 layers Au & 1 layer Ag & 2 layers Ag & 3 layers Ag & 4 layers Ag \\
\hline$d_{\mathrm{CO}} / \AA$ & 1.15 & 1.14 & 1.15 & 1.15 & 1.15 \\
$d_{\mathrm{C}-\mathrm{M}} / \AA$ & 2.05 & 2.20 & 2.24 & 2.25 & 2.30 \\
$\theta /^{\circ}$ & 0.5 & 0.7 & 4.9 & 7.5 & 19.5 \\
$q_{\mathrm{C}} / e^{-}$ & 0.270 & 0.155 & 0.150 & 0.148 & 0.132 \\
$q_{\mathrm{O}} / e^{-}$ & -0.031 & -0.046 & -0.051 & -0.052 & -0.050 \\
\hline
\end{tabular}

After I had determined the minimum adsorption structures for the different surfaces I used them for the following calculations to get an expression for the dipole moment operator and to get parameters to describe the wave functions from a Morse potential. To obtain both of them I calculated the energy and the atomic charges for different $\mathrm{CO}$ bond distances from 1.05 to $1.30 \AA$, keeping the slab atoms and the COM of the molecule fixed at their optimized positions. For these calculations, I also used the tight-settings. The Morse parameters, the stiffness of the function $\alpha_{\mathrm{M}}$, the equilibrium bond distance $r_{\text {eq }}$ and the dissociation energy $\mathrm{D}_{\mathrm{e}}$ obtained from the fits to the calculated energy values and are represented in Tab. 11. Only $r_{\text {eq }}$ shows a decrease when the number of Ag-layers is increased. All fit parameters are different to their values of the free CO molecule. Tus $r_{\text {eq }}$ is longer and the dissociation energy is lower when the molecule sits in its minimum position at the surface. 
Tab. 11: Morse parameters $\alpha_{\mathbf{M}}, r_{\mathbf{e q}}, D_{\mathbf{e}}$ and parameters from the function in Eq. (72) to describe the transition dipole moment operator for $\mathrm{CO}$ and $\mathrm{CO}$ at different $\mathrm{p}(3 \times 3)$ slab with 4 layers.

\begin{tabular}{ccccccc}
\hline System & $\mathrm{CO}$ & 4 layers Au & 1 layer Ag & 2 layers Ag & 3 layers Ag & 4 layers Ag \\
\hline$r_{\mathrm{eq}} / \AA$ & 1.140 & 1.152 & 1.150 & 1.148 & 1.147 & 1.145 \\
$\alpha_{\mathrm{M}} / \AA^{-1}$ & 2.343 & 2.400 & 2.447 & 2.455 & 2.452 & 2.444 \\
$D_{\mathrm{e}} / \mathrm{eV}$ & 10.199 & 8.685 & 8.333 & 8.340 & 8.459 & 8.644 \\
\hline$a / 10^{-3} \mathrm{D} \AA^{-5}$ & 61.19 & 11.21 & 15.23 & 13.86 & 13.34 & 21.84 \\
$b / 10^{-3} \mathrm{DA}^{-4}$ & -13.87 & 3.89 & 3.48 & 3.70 & 3.68 & 0.90 \\
$c / 10^{-3} \mathrm{D}^{-3}$ & 4.32 & 6.44 & 5.78 & 6.06 & 6.15 & 3.70 \\
$d / 10^{-5} \AA^{-2}$ & 0.02 & 1.48 & 0.02 & 0.80 & 1.77 & 1.66 \\
\hline
\end{tabular}

From the Mulliken population analysis of these calculations I computed the total polarization per surface area, which is an equivalent to the dipole moment in periodic systems, for every configuration. Furthermore, as the change in polarization is independent of the system size, I determined it with respect to dipole moment per area (polarization) of the relaxed structure of the adsorbed molecule on the surface. In Fig. 64 this quantity is plotted as a function of the CO bond length with respect to $r_{\text {eq. }}$. The fits applied to these data are used as approximated expressions for the dipole moment operator. Although the data for the slabs show a linear behavior a cubic fit was applied to them as such a fit gives a better match to the free CO.

From these fits, the parameters of which are given in Tab. 11, and the wave functions determined by the parameters in the Morse function (see Tab. 11 and Eq. (70)), the matrix elements for the desired transition dipole moment can be computed.

Tab. 12: Dipole matrix elements $\langle n|\widehat{\mu}| m\rangle$ in $\times 10^{-3} \mathrm{D}$ for $\mathrm{CO}$ at different metal surfaces (Au(111), $\mathrm{Ag}(111)$ and Ag-covered Au(111)). The numbers in the head of the table represent the COM-distance of the molecule to the surface $\left(z_{\mathrm{cm}}\right)$ in $\AA$ and the $C O$ coverage is $0.11 \mathrm{ML}$.

\begin{tabular}{ccccccc}
\hline \multicolumn{2}{c}{ System } & $\mathrm{Au}(111)$ & $\mathrm{1Ag} 3 \mathrm{Au}$ & $2 \mathrm{Ag} 2 \mathrm{Au}$ & $3 \mathrm{Ag} 1 \mathrm{Au}$ & $\mathrm{Ag}(111)$ \\
$n$ & $m$ & 2.72 & 2.82 & 2.86 & 2.84 & 2.81 \\
\hline 0 & 0 & 3.347 & 2.153 & 1.689 & 1.037 & 1.065 \\
0 & 1 & 15.890 & 14.289 & 14.936 & 15.107 & 9.172 \\
0 & 2 & -0.438 & -0.396 & -0.419 & -0.432 & -0.367 \\
1 & 1 & 8.150 & 6.588 & 6.327 & 5.693 & 2.757 \\
1 & 2 & 22.923 & 20.699 & 21.607 & 21.836 & 13.387 \\
\hline
\end{tabular}

In Tab. 12 the dipole moment matrix elements for different transitions for the above mentioned systems are summarized. The transition dipole moment of the moleculesurface system is largest at the $\mathrm{Au}(111)$ and decreases when the number of $\mathrm{Ag}(111)$ layers is increased. The overtone transition from $0 \rightarrow 2$ is negative at all surfaces. Furthermore, 

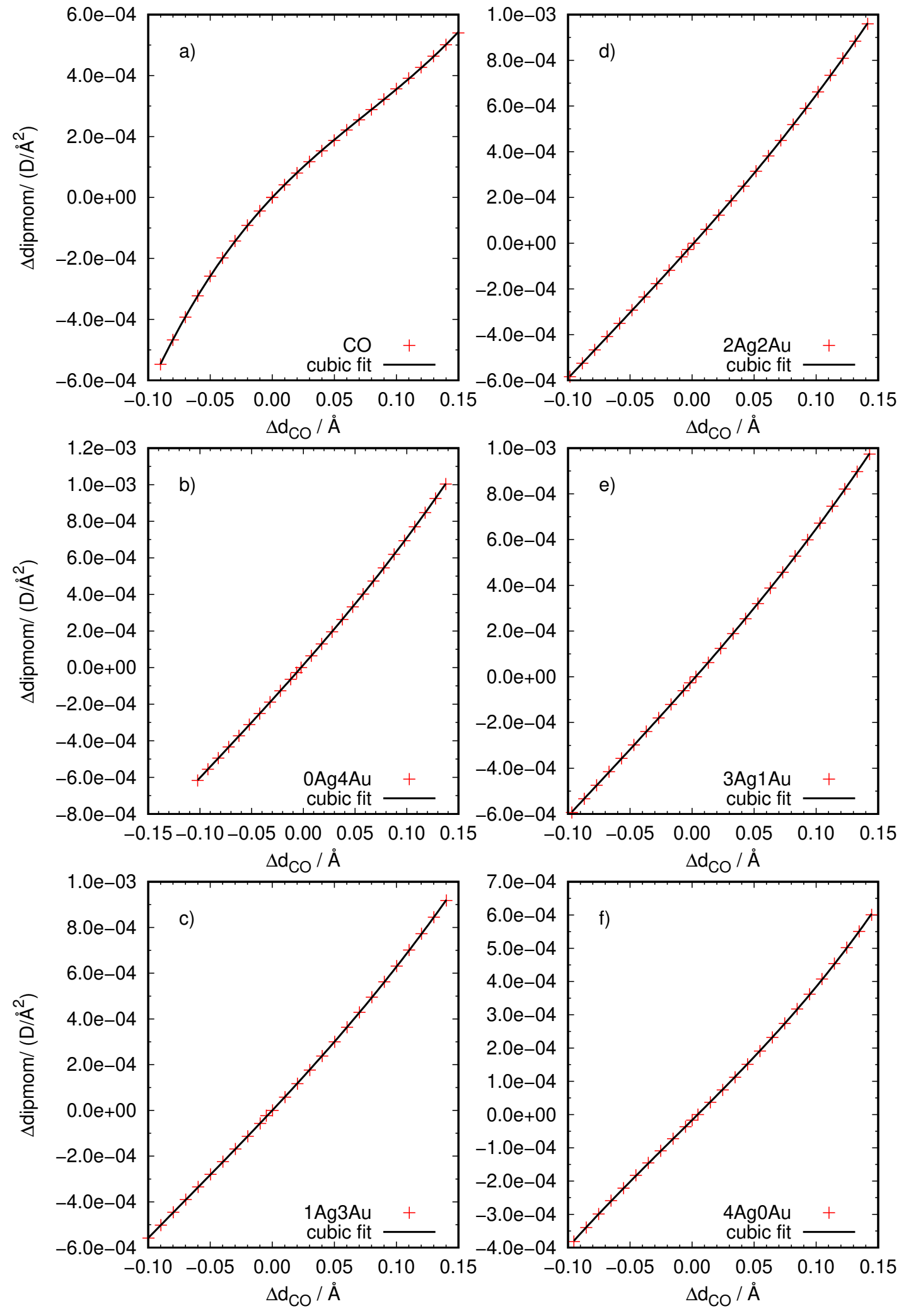

Fig. 64: Change in dipole moment $\Delta$ dipmom as function of the displacement of the $\mathrm{CO}$ bond $\Delta r_{\mathrm{CO}}$ for the free CO molecule (a) and different metal slabs: Au(111) (b), 1 layer Ag (c), 2 layers Ag (d), 3 layers Ag (e), and Ag(111) (f). 
the transition dipole moment from $1 \rightarrow 2$ is about $\sqrt{2} \times$ larger than this from $0 \rightarrow 1$. This is again due to the more harmonic behavior of the Morse potential when the molecule is in low vibrational states.

\section{$9.4 \mathrm{CO}$ on $\mathrm{NaCl}(100)$}

To calculate the transition dipole moment of $\mathrm{CO}$ on this insulating surface I used the same procedure as described in the previous section. But I used a slightly different set of parameters in the control. in-file. I especially used a $\sigma$ of $0.01 \mathrm{eV}$ for the Gaussian smearing function to model the electronic occupation of insulating surface. The surface was modeled by a $c(2 \times 1)$-cell — so a square built up by two $\mathrm{Na}$ and two $\mathrm{Cl}$ atoms with 4 layers in a way that each $\mathrm{Na}$ atom is surrounded by $\mathrm{Cl}$ atoms. The atoms of the bottom layer were kept fixed on their equilibrium positions which were determined by an optimized lattice constant of $5.65 \AA$. This constant was determined like the values for (111)-surfaces, as described in subsection 4.1.2.1. For the (100)-cut the interlayer distance equals the lattice constant. The geometry.in-files used to represent the $\mathrm{NaCl}(100)$ surfaces are shown in Fig. A.15. The simulation cell is further described by a vacuum distance of $20.0 \AA$. To obtain the optimized structure of the molecule at the insulator I did a geometry optimization of the structure until all forces acting on the atoms are smaller than $0.01 \mathrm{eV}^{-1}$. From the found minimum energy configuration the $\mathrm{CO}$ bond length was varied along the bond axis to determine the Morse-parameters as well as the polarization as function of the bond length. The corresponding fit parameters can be found in Tab. 13. The parameters of the Morse fit are nearly the same for the different CO coverage as well as for the different distances of the molecule to the surface. Only $D_{\text {e }}$ per molecule is about $0.1 \mathrm{eV}$ higher for the higher CO coverage (1 ML) than for the lower one $(0.5 \mathrm{ML})$. With these two quantities the transition dipole moment was determined as described in the previous section.

To see how the transition dipole moment is affected by the slab I did these calculations for different distances of the molecular COM to the slab. For the coverage dependence of the quantity I did calculations with two CO molecules sitting at their equilibrium positions above $\mathrm{Na}$ atoms in the same cell as described above.

The computed matrix elements of the dipole moment for the different conditions are shown in Tab. 14. The elements for the first transition - from $|0\rangle$ to $|1\rangle-$ increase when the distance between the molecule and the slab is increased. If the CO coverage is doubled the transition dipole moment is nearly $2 \times$ higher. The overtone transition gives a negative moment for all investigated configurations and shows an increasing absolute value when the $\mathrm{CO}$ is further away from the surface. This is in contrast to the 
Tab. 13: Parameters $r_{\mathbf{e q}}, \alpha_{\mathrm{M}}$ and $D_{\mathbf{e}}$ of the Morse potential (top) and those of the fit function (bottom, see Eq. (72)) to present the dipole moment operator for $\mathrm{CO}$ at $\mathrm{NaCl}(100)$ at different distances of the molecule to the surface $\left(z_{\mathrm{cm}}\right)$ and $\mathrm{CO}$ coverage.

\begin{tabular}{lccccccccc}
\hline CO coverage/ ML & 0.5 & 1 & 0.5 & 1 & 0.5 & 0.5 & 0.5 & 0.5 & 0.5 \\
$z_{\mathrm{cm}} / \AA$ & 3.41 & 3.46 & $3.47^{\mathrm{a}}$ & $3.32^{\mathrm{a}}$ & 4.00 & 5.00 & 6.00 & 7.00 & 10.00 \\
\hline$r_{\mathrm{eq}} / \AA$ & 1.139 & 1.140 & 1.139 & 1.140 & 1.140 & 1.140 & 1.140 & 1.40 & 1.140 \\
$\alpha_{\mathrm{M}} / \AA^{-1}$ & 2.325 & 2.322 & 2.329 & 2.318 & 2.328 & 2.340 & 2.340 & 2.338 & 2.337 \\
$D_{\mathrm{e}} / \mathrm{eV}$ & 10.468 & 10.923 & 10.417 & 10.578 & 10.408 & 10.209 & 10.214 & 10.249 & 10.257 \\
\hline$a / 10^{-3} \mathrm{D}^{-5}$ & 88.11 & 169.48 & 85.64 & 174.14 & 55.46 & 72.33 & 67.35 & 63.26 & 61.23 \\
$b / 10^{-3} \mathrm{D}^{-2}$ & -8.99 & -20.19 & -8.76 & -20.67 & -7.54 & -11.60 & -13.47 & -14.33 & -14.59 \\
$c / 10^{-3} \mathrm{D} \AA^{-3}$ & 2.50 & 4.62 & 2.40 & 4.10 & 3.32 & 3.69 & 4.29 & 4.28 & 4.44 \\
$d / 10^{-6} \mathrm{D} \AA^{-2}$ & -1.34 & -0.10 & -1.04 & 0.25 & 22.51 & 1.90 & 1.62 & 1.03 & 0.83 \\
\hline $\mathrm{a}: c(2 \times 1)$-cell (rotated by $\left.45^{\circ}\right)$ & & & & & & & \\
\hline
\end{tabular}

Tab. 14: Dipole matrix elements $\langle n|\widehat{\mu}| m\rangle \times 10^{-3} \mathrm{D}$ of $\mathrm{CO}$ on $\mathrm{NaCl}(100)$ slabs for two different molecular coverage and distance of the molecule to the slab $\left(z_{\mathrm{cm}}\right.$ in $\left.\AA\right)$, represented by the numbers in the second line of the table head.

\begin{tabular}{lcccccccccc}
\hline \multicolumn{2}{l}{ CO coverage/ ML } & 0.5 & 1 & 0.5 & 1 & 0.5 & 0.5 & 0.5 & 0.5 & 0.5 \\
$n$ & $m$ & 3.41 & 3.46 & $3.47^{a}$ & $3.32^{a}$ & 4.00 & 5.00 & 6.00 & 7.00 & 10.00 \\
\hline 0 & 0 & -0.010 & -0.075 & -0.005 & -0.148 & -0.553 & 0.144 & 0.140 & 0.084 & 0.086 \\
0 & 1 & 2.937 & 5.421 & 2.837 & 4.843 & 3.640 & 4.153 & 4.769 & 4.720 & 4.880 \\
0 & 2 & -0.504 & -1.112 & -0.491 & -1.094 & -0.519 & -0.739 & -0.880 & -0.929 & -0.954 \\
1 & 1 & 0.207 & 0.060 & 0.200 & -0.176 & -0.130 & 0.425 & 0.417 & 0.272 & 0.289 \\
1 & 2 & 4.521 & 8.314 & 4.371 & 7.509 & 5.347 & 6.095 & 6.898 & 6.784 & 6.992 \\
\hline \multicolumn{2}{l}{$c(2 \times 1)$-cell (rotated by $\left.45^{\circ}\right)$} \\
\hline
\end{tabular}


transition dipole moments calculated for the system $\mathrm{CO} / \mathrm{Au}(111)$, where the moment decreases when the molecule is further away from the surface. The expectation value for the dipole moment in the ground state shows a negative sign when the molecule is close to the slab, but the value is positive when $z_{\mathrm{cm}}$ is above $4.00 \AA$. The transition dipole moment $\mu_{01}$ for a $\mathrm{CO}$ mono-layer on $\mathrm{NaCl}(100)$ was recently determined experimentally to be $0.079 \mathrm{D}$ [168]. This value is one magnitude larger than the calculated value of $5.42 \times 10^{-3} \mathrm{D}$.

\subsection{Conclusions}

The calculated matrix elements of the transition dipole moment for $\mathrm{CO}$ at the metal surfaces are about 1-2 magnitude(s) larger than the values calculated for $\mathrm{CO}$ in vacuum in the case of the calculations done with FHI-aims. The obtained values from the calculations performed with VASP show similar magnitudes for $\mathrm{CO}$ at $\mathrm{Au}(111)$ and the molecule in vacuum, whereas the latter case shows always negative values and differs strongly from the values obtained with FHI-aims. Since both values of the dipole moment of the molecule in vacuum computed via either Mulliken or Bader charges differ strongly from the value experimentally determined, we cannot make a clear statement about which charge analysis program is better. Perhaps one could argue that Mulliken-analysis is more suitable for such calculations than Bader analysis because the transition dipole moment for the $0 \rightarrow 1$ transition of $\mathrm{CO}$ at $\mathrm{Au}(111)$ and $\mathrm{CO}$ in vacuum differs more in the former analysis than in the latter, which is also observed in experimental investigations. Of course, the difference between the calculated and the experimental value can also be due to the DFT calculations or the used XC functional. Even more important is presumably the approximation that the wave function of the molecule at the surface does not differ strongly from that of the molecule in vacuum.

However, the transition dipole moment matrix elements of the different metal surfaces do not show large differences between the $\mathrm{Au}(111)$ surface and the surface covered with 1 ML Ag. Thus, it seems to be unlikely that differences in the electric properties of the molecule cause the differences in the translational energy distribution of CO scattered from these surfaces. Therefore, an explanation with a change of the mechanical properties seems to be more reasonable as discussed in the conclusions of chapter 5 . In the case of $\mathrm{CO}$ at $\mathrm{NaCl}(100)$ the matrix elements for a $0 \rightarrow 1$ transition are smaller than those of the metal (111)-surfaces. An increase of the CO coverage increases the transition dipole moment for all investigated surfaces. The values of the dipole moment for the molecule in vacuum are much smaller than the one experimentally determined, 
whereas the values for $\mathrm{CO}$ at $\mathrm{NaCl}(100)$ seem to agree better than those of the molecule in vacuum.

In a further perspective it would be possible for the insulators to calculate the polarization of the system via a different method based on BERRY phases [169], thus directly from the wave function of an $a b$-initio calculation. With this method one could further classify the results obtained here and the method developed here to compute the transition dipole matrix elements as well as get a better comparison to the experimentally determined results. However, it is not possible to calculate the polarization of metallic or rather conducting systems, and therefore the method developed here can give some evidence on how the transition dipole moment changes when the molecule is close to a surface.

Finally, the method of the calculation of the transition dipole moment may give some insights into the differences of the vibrational lifetime of the molecule at the surface. The lifetime is much shorter when CO sits at the $\mathrm{Au}(111)$-surface (49 ps [165]) than the value on $\mathrm{CO}$ on $\mathrm{NaCl}(100)$ (4.3 ms [26]). This may be also due to the smaller transition dipole moment in the case of $\mathrm{CO}$ at the insulator. But as the difference between the $0 \rightarrow 1$ transition for the metal and insulator surface is only small and therefore also non-adiabatic effects may also play a prominent role in the interaction which causes the short vibrational lifetime of the molecule at metal surfaces. 


\section{Discussion}

In summary, we have seen that DFT calculations can provide insights in a variety of different aspects, which can influence the energy transfer between a scattered molecule and a metal surface. These aspects comprise mechanical and electronic properties of the surface (see chapters 5 and 9) but also the interaction energy between the molecule and the surface (see chapter 7). From the mechanical properties we could see that a change in the phonon spectra when dosing an $\mathrm{Au}(111)$ surface with layers of $\mathrm{Ag}$ influences the translational energy distribution for CO scattered from those surfaces. The interaction energy between molecule and a surface cannot explain these differences in the energy distribution of the scattered molecules, because the PES profiles show only small differences (see section 7.3). The investigation of the transition dipole moment may give evidence for the different vibrational lifetimes of $\mathrm{CO}$ on metal and insulator surfaces. However, the calculated values differ strongly from the existing experimental data for the CO molecule in vacuum. Therefore, further investigations seem to be necessary to go to the bottom of this issue.

Furthermore, we can look at reactions of diatomic molecules on surfaces and make predictions about which kind of energy promotes a reaction and how the energy is distributed among the dofs of the products (see chapter 6). The investigated dissociation reactions of the hydrogen halides ( $\mathrm{HF}$ and $\mathrm{HCl}$ ) and $\mathrm{NO}$ on (111)-surfaces of fcc metals all show a late barrier, and thus the reactions should be promoted by vibrational excitation of the diatomic molecules. The dissociation barriers for $\mathrm{HCl}$ on $\mathrm{Au}(111)$ and $\mathrm{Ag}(111)(0.93 \mathrm{eV}$ and $0.80 \mathrm{eV})$ are about $50 \%$ lower than those of HF on $\mathrm{Au}(111)(1.83 \mathrm{eV})$ and $\mathrm{Ag}(111)(1.53 \mathrm{eV})$. Only in the case of $\mathrm{HCl}$ on $\mathrm{Ag}(111)$ are the products $(\mathrm{H}$ and $\mathrm{Cl}$ separated on the surface) more stable than the reactant ( $\mathrm{HCl}$ on the surface) and on the silver surface the dissociation barrier is lower than in case of the gold surface. Since the barrier of $\mathrm{HCl} / \mathrm{Au}(111)$ seems to be much lower than in the experimental investigations, this suggests that more theoretical effort is needed to explain the differences. Therefore simulations which go beyond the BOA should be done on this system. The MEP calculations of $\mathrm{NO}$ on $\mathrm{Au}(111), \mathrm{Ag}(111)$, and $\mathrm{Cu}(111)$ reveal that the dissociation on $\mathrm{Cu}(111)$ (the least noble surface) has the lowest barrier ( $1.8 \mathrm{eV})$, whereas the barrier on $\mathrm{Au}(1111)$ and $\mathrm{Ag}(111)$ are about two times higher $(3.4 \mathrm{eV}$ and $3.2 \mathrm{eV})$. The product on $\mathrm{Cu}(111)$ is 
about $2 \mathrm{eV}$ more stable than on the two other surfaces. Thus, the dissociation of NO on the copper surface is clearly more probable than on the counterparts on the gold and silver surfaces.

The TS configuration of the CO oxidation on $\mathrm{Pt}(111)$ looks more like the reactants, and thus the product should be vibrationally excited. The barrier height of the reaction increases about $0.1 \mathrm{eV}$ when the $\mathrm{CO}$ and $\mathrm{O}$ coverage is increased from 0.11 to $0.25 \mathrm{ML}$. From AIMD calculations we can conclude that this happens because the formed $\mathrm{CO}_{2}$ has a lot of energy in its vibrational dofs. Furthermore, the final translational energy distribution of the product shows differences to the experimental findings, and this seems to be due to non-adiabatic effects [38].

Moreover, with AIMD simulations, we can find evidence on how strong the energy transfer between molecule and surface in molecule-surface scattering is influenced by non-adiabatic effects. For this purpose, we have to compare the results of the simulations with corresponding experimental ones as done in chapter 8 . The scattering of CO in low vibrational states $(\nu=0,2)$ shows qualitative agreement between the results of simulations and the experiment. In particular, the simulations done with RPBE-functional agree with the experimentally obtained final translational energy distribution (see Fig. 46). Thus, non-adiabatic effects seem to play a negligible role in the energy transfer between the molecule and the surface. The translational energy of the impinging molecule is transfered into the rotational dofs of the molecule and into the slab, whereas no vibrational energy is gained or lost when the molecule is scattered from one of the considered surfaces.

In the case of AIMD simulations of highly vibrationally excited CO scattering a variety of different tests and a switch from VASP to FHI-aims DFT-code was necessary to obtain trajectories with an acceptable drift in the total energy. In the vibrationally elastic channel the final translational energy distributions of $\mathrm{CO}(v=17)$ scattered from $\mathrm{Au}(111)$ and $\mathrm{Ag}(111)$ obtained from the adiabatic simulations are in agreement with those of the experiment. Thus, in the case if vibrational energy is not exchanged during the scattering, non-adiabatic effects apparently play a minor role in the transfer of the translational energy to either rotational dofs of the molecule or to the phonon bath of the surface. For the vibrationally inelastic channel the calculations show differences between $\mathrm{Au}(111)$ and $\mathrm{Ag}(111)$. In the case of $\mathrm{Au}(111)$ the final vibrational state distributions of the scattered CO show a qualitative agreement, whereas in the case of the $\mathrm{Ag}(111)$ surface the discrepancies are larger. Hence, we can conclude that the energy transfer between the molecule and the surface seems to be more influenced by non-adiabatic effects in the case of $\mathrm{Ag}(111)$ than in the case of $\mathrm{Au}(111)$. An explanation of this difference can be due to the lower work function of $\mathrm{Ag}(111)$, so it is easier to remove an electron 
from the silver surface than from the gold surface. Scattering simulations of $\mathrm{CO}(v=22)$ from $\mathrm{Au}(111)$ and $\mathrm{Ag}(111)$ show more vibrational relaxation than the simulations for $\mathrm{CO}(v=17)$. There is no evidence for the dissociation of the molecule during the scattering event on both surfaces. But the initial vibrational energy of CO molecule in $v=22$ is about $5.2 \mathrm{eV}$ which is much smaller than the dissociation energy of the molecule in the gas phase of about $12 \mathrm{eV}$. Therefore, even if the dissociation barrier is lowered when the molecule interacts with the metal surface, simulations of CO in even higher initial $v$-state seem to be necessary to observe the dissociation process. But before doing this, MEP calculations for this process on $\mathrm{Au}(111)$ and $\mathrm{Ag}(111)$ should be done to shed light on the barrier height. In a further perspective the DFT data obtained from these simulations can be used as an input to fit a high-dimensional PES for $\mathrm{CO} / \mathrm{Au}(111)$ and $\mathrm{CO} / \mathrm{Ag}(111)$.

The AIMD simulations for $\mathrm{NO}(v=16)$ scattered from $\mathrm{Au}(111)$ disagree strongly with the experimentally found final vibrational state distribution of the scattered molecules. Here, much more vibrational relaxation is observed in the experiment than obtained from the calculated distribution. Hence, the transfer of vibrational energy during the scattering is likely to be governed by non-adiabatic effects, like ehp excitation. Since the simulations for $\mathrm{NO}(v=16) / \mathrm{Ag}(111)$ give similar results for the vibrational state distribution these effects may play an important role in this system too. To further investigate these effects, simulations need to be done which go beyond the BOA and include not only the electronic ground state PES. The IESH approach [46] is preferable to use because it was successful in describing some experimental observations [47], but failed to describe all of them [48]. This disagreement seems to be due to the used diabatic potential which is not accurate enough. The reason for that is likely to be related with the difficulty to control the magnetic moment of the system when doing DFT calculations. More recently, a developed PES for NO/Au(111), produced via a high-dimensional neural network [49] by YIN et al. [50], gives better agreement with the scattering experiments, but here non-adiabatic effects were not included. Therefore, the DFT data produced in this work can be considered as the first step on the route to a new diabatic potential including a neutral (ground) state and an anionic (excited) state with $\mathrm{NO}$ and $\mathrm{NO}^{-}$, respectively. To check if the data are converged to correct spin state one could analyze the spin densities via the Bader approach and see if there is one unpaired electron on the molecule and if this is not the case exclude the data from further considerations. The second step is the fitting of these data to obtain the PES. This can be carried out by either following the route of ROY and co-workers [157] or using a neural network. The third step is to perform simulations within the IESH approach with this new potential and see if the outcome gives a better agreement with 
the experimental findings than the previous simulations. Furthermore, to find out when the electron transfer and thus non-adiabatic effects start to play a dominant role, AIMD simulations for $\mathrm{NO}(v=3) / \mathrm{Au}(111)$ should be carried out, because here the descriptor for the electron transfer $(\mathrm{VEBE}-\Phi)$ is between the values of the descriptor in the investigated systems (see Fig. 62 and [43]).

To investigate the dissociation of impinging highly vibrationally excited molecules at a metal surface, a good system to start with would be $\mathrm{NO} / \mathrm{Cu}(111)$ because the dissociation barrier of this system is relatively low $(\approx 1.8 \mathrm{eV})$ compared to that on $\mathrm{Au}(111)$ $(\approx 3.3 \mathrm{eV})$ and thus the molecule has to be in a not so high vibrational state to overcome this barrier, e.g. the vibrational energy of the molecule in $v=13$ is about $2.9 \mathrm{eV}$ and thus $1 \mathrm{eV}$ higher than the dissociation barrier. Therefore, performing AIMD simulations of $\mathrm{NO}(v=13) / \mathrm{Cu}(111)$ is a good choice to study if dissociation occurs in this system, especially as experimental evidence for this was observed [13, 154]. A small number of trajectories for this system was carried out and reveal that about $0.25 \%$ of the trajectories show dissociation (see section 6.5).

Finally, we have seen that AIMD simulations are an excellent tool to study the energy transfer between an impinging molecule and a metal surface. In particular, when compared to scattering experiments we can obtain evidence on how strong the energy transfer is affected by non-adiabatic effects. Furthermore,the results of AIMD simulations do not contain errors due to the fitting procedure employed to produce the PES used for MD simulations. Moreover, we can study reactions of small molecules to shed light on the role of non-abiabatic effects during the reaction. For the CO-oxidation on $\mathrm{Pt}(111)$ this role seems to be important but even more important in the case of the dissociation of highly vibrationally excited $\mathrm{NO}$ on $\mathrm{Cu}(111)$, where the preliminary results of AIMD simulations strongly disagree with experimental observations. 


\section{Bibliography}

[1] R. Brent, The golden book of chemistry experiments, 1st, Golden Press, New York, 1960, p. 114.

[2] M. Planck, Wissenschaftliche Selbstbiographie, 1st, Johann Ambrosius Verlag, Leipzig, 1948, p. 33.

[3] G. Ertl in, 2000, pp. 1-69, https : //linkinghub . elsevier . com/retrieve/ $\mathrm{pii/S0360056402450122.}$

[4] B. Hammer, J. Nørskov in, 2000, pp. 71-129, https://linkinghub. elsevier. com/retrieve/pii/S0360056402450134.

[5] X. Yang, A. M. Wodtke, Chem. Soc. Rev. 2016, 45, 3573.

[6] A. M. Wodtke, Chem. Soc. Rev. 2016, 45, 3641.

[7] K. Ziegler et al., Angew. Chemie Oct. 1955, 67, 541, http : //doi . wiley . com/ 10.1002/ange.19550671902.

[8] A. Logadottir et al., J. Catal. 2001, 197, 229.

[9] J. Greeley et al., Nat. Mater. 2006, 5, 909.

[10] A. J. Medford et al., J. Catal. 2015, 328, 36, http://dx . doi .org/10 .1016/ j . jcat. 2014.12.033.

[11] C. T. Rettner et al., Phys. Rev. Lett. 1985, 55, 1904.

[12] B. D. Kay, T. D. Raymond, M. E. Coltrin, Phys. Rev. Lett. 1987, 59, 2792.

[13] H. Hou et al., Science (80-. ). 1999, 284, 1647.

[14] E. K. Watts, J. L. Siders, G. O. Sitz, Surf. Sci. 1997, 374, 191.

[15] Y. Huang et al., Science (80-. ). 2000, 290, 111.

[16] R. Cooper et al., Chem. Sci. 2010, 1, 55.

[17] R. Cooper et al., Angew. Chemie - Int. Ed. 2012, 51, 4954.

[18] R. Cooper et al., J. Chem. Phys. 2012, 137, DOI 10.1063/1. 4738596.

[19] K. Golibrzuch et al., J. Phys. Chem. A 2013, 117, DOI 10.1021/jp403382b. 
[20] T. Schäfer et al., Chem. Phys. Lett. 2012, 535, 1, https://linkinghub. elsevier . com/retrieve/pii/S0009261412003673.

[21] N. Bartels et al., Angew. Chemie - Int. Ed. 2014, 53, 13690.

[22] N. Bartels et al., J. Chem. Phys. 2014, 140, 0.

[23] J. D. White et al., J. Vac. Sci. Technol. A Vacuum Surfaces Film. 2005, 23, 1085.

[24] J. D. White et al., Nature Feb. 2005, 433, 503, http : / / www . nature . com / articles/nature03213.

[25] M Morin, N. J. Levinos, A. L. Harris, J. Chem. Phys. 1992, 3950.

[26] H. C. Chang, G. E. Ewing, Phys. Rev. Lett. 1990, 65, 2125.

[27] S. M. Janke et al., Zeitschrift für Phys. Chemie Nov. 2013, 227, 1467, http : / / www . degruyter . com/view/j/zpch . 2013. 227 . issue-9-11/zpch-20130411/zpch-2013-0411.xml.

[28] H. Nienhaus et al., Phys. Rev. Lett. 1999, 82, 446.

[29] M. Born, R. Oppenheimer, Ann. Phys. 1927, 389, 457.

[30] G.-J. J. Kroes, C. Díaz, Chem. Soc. Rev. 2016, 45, 3658, http : / / xlink . rsc . org $/$ ?DOI=C5CS00336A.

[31] F. Nattino et al., Phys. Rev. Lett. 2012, 108, 1.

[32] F. Nattino et al., J. Chem. Phys. 2014, 141, DOI 10 . 1063/1 . 4896058, http: //dx.doi.org/10.1063/1.4896058.

[33] F. Nattino et al., J. Phys. Chem. Lett. 2014, 5, 1294.

[34] X. J. Shen et al., Phys. Rev. Lett. 2014, 112, 1.

[35] P. R. Shirhatti et al., J. Phys. Chem. Lett. 2016, 7, 1346.

[36] T. Liu, B. Fu, D. H. Zhang, J. Chem. Phys. 2013, 139, DOI 10. 1063/1. 4829508.

[37] J. C. Polanyi, Acc. Chem. Res. 1972, 5, 161.

[38] J. Neugebohren et al., Nature 2018, 558, 280.

[39] C. Steinsiek et al., J. Phys. Chem. C 2018, 122, 18942.

[40] P. R. Shirhatti et al., J. Chem. Phys. 2014, 141, DOI 10.1063/1.4894814, http: //dx.doi.org/10.1063/1.4894814.

[41] C. Steinsiek et al., J. Phys. Chem. C 2018, 122, 10027.

[42] R. Wagner et al., J. Phys. Chem. Lett. 2017, 8, DOI 10 . 1021/acs . jpclett . $7 \mathrm{~b} 02207$. 
[43] R. J. Wagner et al., Phys. Chem. Chem. Phys. 2019, 21, 1650.

[44] N. Bartels et al., Proc. Natl. Acad. Sci. U. S. A. 2013, 110, 17738.

[45] J. C. Tully, J. Chem. Phys. 1990, 93, 1061, http://aip.scitation.org/doi/ $10.1063 / 1.459170$.

[46] N. Shenvi, S. Roy, J. C. Tully, Science (80-. ). 2009, DOI 10 . 1126 / science . 1179240.

[47] N. Shenvi, S. Roy, J. C. Tully, J. Chem. Phys. 2009, 130, DOI 10 . 1063 / 1 . 3125436.

[48] K. Golibrzuch et al., J. Chem. Phys. 2014, 140, DOI 10.1063/1.4861660.

[49] J. Behler, J. Chem. Phys. 2011, 134, DOI 10.1063/1.3553717.

[50] R. Yin, Y. Zhang, B. Jiang, J. Phys. Chem. Lett. 2019, 10, 5969.

[51] S. Monturet, P. Saalfrank, Phys. Rev. B - Condens. Matter Mater. Phys. 2010, 82, 1.

[52] D. Marx, J. Hutter, Ab Initio Molecular Dynamics, Cambridge University Press, Cambridge, 2009, p. 567, http : / / ebooks . cambridge . org / ref / id / CB09780511609633.

[53] M. Baer, Beyond Born-Oppenheimer: Electronic Nonadiabatic Coupling Terms and Conical Intersections, 2006.

[54] F. Jensen, Introduction to Computational Chemistry, Second, Wiley \& Sons, Chichester, 2009, p. 599.

[55] W. Koch, M. C. Holthausen, A Chemist's Guide to Density Functional Theory, 2nd ed., Wiley, 2001, p. 294, http : / / doi . wiley . com / 10 . 1002 / 3527600043https : / / onlinelibrary.wiley . com/doi/book/10 . 1002/ 3527600043.

[56] D. Frenkel, B. Smit, Understanding Molecular Simulation: From Algorithms to Applications, 1st ed., Academic Press, San Diego, 1996.

[57] M. Born, K. Huang in, Clarendon Press, New York, 1954, Chapter IV, p. 432.

[58] J. Tully, Annu. Rev. Phys. Chem. 2000, 51, 153, http: //www . annualreviews . org/doi/pdf/10.1146/annurev . physchem.51.1.153.

[59] H. Nakamura, Nonadiabatic transition: Concepts, basic theories and applications, 2nd ed., 2012.

[60] P.-O. Löwdin, Int. J. Quantum Chem. 1985, 28, 19. 
[61] P. Hohenberg, W. Kohn, Phys. Rev. 1964, 136, 864.

[62] E. Fermi, Zeitschrift für Phys. 1926, 36, 902.

[63] P. Dirac, Proc. R. Soc. London. Ser. A 1926, 112, 661.

[64] W. Pauli, Zeitschrift für Phys. 1925, 31, 765.

[65] E. Engel, R. M. Dreizler, Density Functional Theory, Springer Berlin Heidelberg, Berlin, Heidelberg, 201 1, p. 540, http : //link . springer . com/10 . 1007/9783-642-14090-7.

[66] H. Hellmann, Einführung in die Quantenchemie, (Ed.: D. Andrae), SpringerVerlag, Berlin, Heidelberg, 2015, pp. 19-376, http://link . springer . com/10 . 1007/978-3-662-45967-6\{\_\}2.

[67] L. H. Thomas, Math. Proc. Cambridge Philos. Soc. 1927, 23, 542.

[68] E. Fermi, Zeitschrift für Phys. 1928, 48, 73.

[69] F. Bloch, Zeitschrift für Phys. 1929, 57, 545.

[70] P. A. Dirac, Math. Proc. Cambridge Philos. Soc. 1930, 26, 376.

[71] W. Kohn, L. J. Sham, Phys. Rev. 1965, 140, A1133.

[72] J. C. Slater, Phys. Rev. 1951, 81, 385.

[73] E. Wigner, Phys. Rev. 1934, 46, 1002.

[74] D. M. Ceperley, B. J. Alder, Phys. Rev. Lett. 1980, 45, 566.

[75] S. H. Vosko, L. Wilk, M. Nusair, Can. J. Phys. 1980, 58, 1200.

[76] J. P. Perdew in Electron. Struct. Solids '91, (Eds.: P Ziesche, H Eschrig), Akademie Verlag, Berlin, 1991, pp. 11-20.

[77] C. F. Weizsäcker, Zeitschrift für Phys. 1935, 96, 431.

[78] A. D. Becke, Phys. Rev. A 1988, DOI 10.1103/PhysRevA.38.3098.

[79] J. Perdew et al., Phys. Rev. B 1992, 46, 6671.

[80] J. P. Perdew, Y. Wang, Phys. Rev. B 1992, 45, 13244, https : // link . aps . org/ doi/10.1103/PhysRevB.45.13244.

[81] Y. Zhang, W. Yang, Phys. Rev. Lett. 1998, 80, 890.

[82] T. W. Keal, D. J. Tozer, J. Chem. Phys. 2004, 121, 5654.

[83] J. P. Perdew, K. Burke, M. Ernzerhof, Phys. Rev. Lett. 1996, 77, 3865.

[84] B. Hammer, L. B. Hansen, J. K. Nørskov, Phys. Rev. B - Condens. Matter Mater. Phys. 1999, 59, 7413. 
[85] C. Lee, W. Yang, R. G. Parr, Phys. Rev. B 1988, DOI 10 . 1103/PhysRevB . 37 . 785.

[86] A. D. Becke, J. Chem. Phys. 1998, 104, 1040.

[87] Y. Zhao, D. G. Truhlar, J. Chem. Phys. 2006, 125, DOI 10.1063/1. 2370993.

[88] A. D. Becke, J. Chem. Phys. 1993, 98, 5648.

[89] P. J. Stephens et al., J. Phys. Chem. 1994, 98, 11623.

[90] F. Bloch, Zeitschrift für Phys. 1929, 52, 555.

[91] N. D. Mermin, Phys. Rev. 1965, 137, 1.

[92] C. L. Fu, K. M. Ho, Phys. Rev. B 1983, DOI 10.1103/PhysRevB. 28.5480.

[93] M. Methfessel, A. T. Paxton, Phys. Rev. B 1989, 40, 3616.

[94] G. Kresse, J. Furthmüller, Comput. Mater. Sci. 1996, 6, 15.

[95] M. E. Tuckerman, Statistical Mechanics: Theory and Molecular Simulation, Oxford University Press, New York, 2010, p. 696.

[96] L. Verlet, Phys. Rev. 1967, 159, 98, https : //link .aps .org/doi/10.1103/ PhysRev.159.98.

[97] W. C. Swope et al., J. Chem. Phys. 1982, 76, 637.

[98] C. W. Gear, Numerical Initial Value Problems in Ordinary Differential Equations, 1st ed., Prentice-Hall, Eaglewood Cliffs, 1971, p. 253.

[99] A. Arnold, N. Mauser, J. Hafner, J. Phys. Condens. Matter 1989, 1, 965.

[100] G. Kresse, J. Hafner, Phys. Rev. B 1993, 47, 558.

[101] M. P. Allen, D. J. Tildesley, Computer Simulation of Liquids, 1st ed., Oxford University Press, Oxford, 1987, p. 385.

[102] H. Hellmann, Zeitschrift für Phys. 1933, 85, 180.

[103] R. P. Feynman, Phys. Rev. 1939, 56, 340.

[104] G. Kresse, D. Vogthuber, M. Marsman, VASP Homepage, 2020, https : //vasp. at (visited on $04 / 22 / 2020$ ).

[105] Fritz Haber Institute ab initio molecular simulations FHI-aims- A Users' Guide, Berlin, 2014, htttp: //www .fhi-berlin.mpg.de/aims/.

[106] A. Togo, Phonopy Homepage, 2009, https ://phonopy.github. io/phonopy/ index.html (visited on 05/28/2020).

[107] L. Chaput et al., Phys. Rev. B - Condens. Matter Mater. Phys. 201 1, 84, 094302. 
[108] G. Henkelman, A. Arnaldsson, H. Jónsson, Comput. Mater. Sci. 2006, 36, 354.

[109] R. F. Bader, Acc. Chem. Res. 1985, DOI 10.1021/ar00109a003.

[110] Methods in Computational Molecular Physics, (Eds.: G. H. F. Diercksen, S. Wilson), Springer Netherlands, Dordrecht, 1983, http : // link . springer . com/ 10.1007/978-94-009-7200-1.

[111] B Liu, Numerical Algorithms in Chemistry: Algebraic Methods, tech. rep., Lawrence Berkley Lab. Univ. of California, 1978, https : / / escholarship . org/uc/item/3xb320bq.

[112] P. Pulay, Chem. Phys. Lett. 1980, 73, 393.

[113] H. J. Monkhorst, J. D. Pack, Phys. Rev. B 1976, DOI 10 . 1103/PhysRevB . 13. 5188.

[114] D. Vanderbilt, Phys. Rev. B 1990, 41, 7892, https ://link .aps .org/doi/10. 1103/PhysRevB.41.7892.

[115] P. E. Blöchl, Phys. Rev. B 1994, 50, 17953.

[116] D. R. Hamann, M. Schlüter, C. Chiang, Phys. Rev. Lett. 1979, 43, 1494.

[117] J. C. Slater, Phys. Rev. 1937, 51, 846.

[118] O. K. Andersen, Phys. Rev. B 1975, 12, 3060, https ://link.aps .org/doi/10. 1103/PhysRevB.12.3060.

[119] D. Joubert, Phys. Rev. B - Condens. Matter Mater. Phys. 1999, 59, 1758.

[120] P. Dirac, Proc. R. Soc. London. Ser. A Contain. Pap. a Math. Phys. Character 1928, 117, 610, https : / /royalsocietypublishing .org/doi/10 .1098/ rspa.1928.0023.

[121] M. F. Herman, E. Kluk, Chem. Phys. 1984, DOI 10 . 1016 / 0301 - 0104 (84) 80039-7.

[122] E. Van Lenthe, E. J. Baerends, J. G. Snijders, J. Chem. Phys. 1994, 101, 9783.

[123] C. G. Broyden, Math. Comput. 1965, 19, 577.

[124] G. P. Kerker, Phys. Rev. B 1981, 23, 3082, https : //link . aps . org/doi / 10. 1103/PhysRevB.23.3082.

[125] W. Tang, E. Sanville, G. Henkelman, J. Phys. Condens. Matter 2009, 21, 84204.

[126] G. Henkelman, H. Jónsson, J. Chem. Phys. 1999, 111, 7010.

[127] G. Henkelman, B. P. Uberuaga, H. Jónsson, J. Chem. Phys. 2000, 113, 9901.

[128] G. Henkelman, H. Jónsson, J. Chem. Phys. 2000, 113, 9978. 
[129] W. E, W. Ren, E. Vanden-Eijnden, J. Chem. Phys. 2007, 126, 164103.

[130] D. Sheppard, R. Terrell, G. Henkelman, J. Chem. Phys. 2008, 128, 134106.

[131] W. E, W. Ren, E. Vanden-Eijnden, Phys. Rev. B - Condens. Matter Mater. Phys. 2002, 66, 523011.

[132] G. Käb, V. S. Vikhrenko, Phys. Chem. Chem. Phys. 2001, 3, 2223.

[133] P. M. Morse, Phys. Rev. 1929, DOI 10.1103/PhysRev.34.57.

[134] J. M. Hollas, J. Chem. Educ. 2005, DOI 10.1021/ed082p43. 1.

[135] W. Heisenberg, Zeitschrift für Phys. 1927, 43, 172.

[136] R. S. Mulliken, J. Chem. Phys. 1955, 23, 1833.

[137] N. A. Spaldin, J. Solid State Chem. 2012, 195, 2.

[138] J. P. Dahl, M. Springborg, J. Chem. Phys. 1988, DOI 10.1063/1. 453761.

[139] E. F. De Lima, J. E. Hornos, J. Phys. B At. Mol. Opt. Phys. 2005, 38, 815.

[140] J. P. Perdew et al., Phys. Rev. Lett. 2008, 100, 1.

[141] C. Kittel, Introduction to Solid State Physics, 8th ed., John Wiley \& Sons, Inc, 2005, p. 680.

[142] B. Kolb, H. Guo, J. Chem. Phys. 2016, 145, 011102, http: / / aip . scitation . org/doi/10.1063/1.4956453.

[143] T. Liu, B. Fu, D. H. Zhang, J. Chem. Phys. 2017, 146, DOI 10.1063/1.4982051, http://dx.doi.org/10.1063/1.4982051.

[144] P. R. Shirhatti et al., J. Phys. Chem. Lett. 2016, 7, 1346.

[145] J. Geweke, A. M. Wodtke, J. Chem. Phys. 2020, 153, DOI 10. 1063/5 .0026228, https://doi.org/10.1063/5.0026228.

[146] J. D. Cox, D. D. Wagman, V. A. Medvedev, CODATA KEY VALUES FOR THERMODYNAMICS, Hemisphere Publishing Corp., New York, 1989, http : // codata . info/resources/databases/key1.html.

[147] Y. Santiago-Rodríguez et al., Surf. Sci. 2014, 627, 57, http://dx.doi .org/10. $1016 / j$.susc. 2014.04 .012$.

[148] B. W. Chen et al., J. Phys. Chem. C 2019, 123, 7551.

[149] N. H. De Leeuw et al., Phys. Rev. B - Condens. Matter Mater. Phys. 2004, 69, 1.

[150] W. Gao et al., J. Am. Chem. Soc. 2008, 130, 3560.

[151] M. Gajdoš, J. Hafner, A. Eichler, J. Phys. Condens. Matter 2006, 18, 41, https : //iopscience.iop.org/article/10.1088/0953-8984/18/1/003. 
[152] A. Tkatchenko, M. Scheffler, Phys. Rev. Lett. 2009, 102, 6.

[153] L. Zhou et al., Angew. Chemie - Int. Ed. 2019, 58, 6916.

[154] H. Hou et al., J. Chem. Phys. 1999, 110, 10660.

[155] D. P. Engelhart et al., Surf. Sci. 2016, 650, 11, http://dx.doi .org/10.1016/j . susc. 2015.06 .010 .

[156] D. Borodin et al., Science (80-. ). 2020, 369, 1461.

[157] S. Roy, N. A. Shenvi, J. C. Tully, J. Chem. Phys. 2009, 130, DOI 10 . 1063 / 1. 3122989.

[158] G. Henkelman, W. Tang, E. Sanville, Code: Bader charge analysis, 20, http: // theory. cm. utexas. edu/henkelman/code/bader/ (visited on 09/12/2019).

[159] B. C. Krüger et al., J. Phys. Chem. Lett. 2016, 7, 441.

[160] F. H. Geuzebroek et al., J. Phys. Chem. 1991, 95, 8409.

[161] M. Marsman, VASP-wiki: I_CONSTRAINED_M, 2019, https : //www . vasp.at/ wiki/index.php/I\{\_\}CONSTRAINED\{\_\}M (visited on 09/27/2020).

[162] D. R. Lide, CRC Handbook of Chemistry and Physics, 84th edition, CRC Press, 2004, p. 2470.

[163] K. M. Refaey, J. L. Franklin, Int. J. Mass Spectrom. Ion Phys. 1976, 20, 19.

[164] H. J. Chen et al., Chinese Phys. B 2015, 24, DOI 10 . 1088/1674-1056/24/8/ 083102.

[165] S. Kumar et al., Phys. Rev. Lett. 2019, 123, 156101, https : / / doi . org / 10 . 1103/PhysRevLett. 123.156101.

[166] A. Liebsch, Phys. Rev. Lett. 1985, 54, 67.

[167] P. Shirhatti et al., Nat. Chem. 2018, DOI 10.1038/s41557-018-0003-1.

[168] A. Schönemann, Bachelor Thesis, Max Planck Institute for Biophysical Chemistry, 2018, p. 82.

[169] M. V. Berry, Proc. R. Soc. London. A. Math. Phys. Sci. 1984, 392, 45. 


\section{A Appendix}

In this appendix a selection of input files for different kinds of calculations carried out with VASP and FHI-aims is presented.

\section{A.1 Example input files for VASP}

The examples comprises the calculations for the energy grid for $\mathrm{NO} / \mathrm{Au}(111)$ and the AIMD simulation of a highly vibrationally excited CO molecule in vacuum. For all examples only the INCAR-, KPOINTS- and the POSCAR-files are presented, whereas the POTCAR-files are neglected. Examples for a bulk calculation to determine the equilibrium lattice constant are shown in section 3.1.

\section{A.1.1 Input files for an AIMD simulation of $\operatorname{CO}(v=17)$ in vacuum}

Here the input files (INCAR, KPOINTS, and POSCAR) for an AIMD simulation of $\mathrm{CO}(v=17)$ in vacuum are shown in Figs. A.1-A.3. These input files were used for calculations as described in section 8.4 to find an input setting which delivers an tolerable drift in the total energy.

\section{A.1.2 Input files for an energy grid calculation for NO/Au(111)}

Exemplary input files for the calculations of the energy grid for $\mathrm{NO} / \mathrm{Au}(111)$ : , INCAR(Fig. A.4), KPOINTS- (Fig. A.5), POSCAR-file (Fig. A.6). The calculations are described in subsection 7.4. 
System CO in vacuum \# comment line

SYSTEM = CO-in-vacuum \# string to describe the system

$\mathrm{GGA}=\mathrm{RP} \# \mathrm{RPBE}$ functional

GGA_COMPAT $=$.FALSE. \# restores symmetry, recommend for GGA

ISYM $=0 \#$ no symmetry considered

IBRION $=0$ \# AIMD simulation requested

SMASS $=-3 \#$ micro canonical (NVE) ensemble

POTIM $=0.02 \#$ time step of $0.02 \mathrm{fs}$

NSW $=5000 \#$ number of time steps

ENCUT $=400 \#$ cut of energy for plane waves

ISMEAR $=1$ \# MP1 smearing function

SIGMA $=0.2 \#$ width of the smearing function

ALGO $=F$ \# fast algorithm for scf-cycle

PREC = ACCURATE \# precision of calculation

NELM $=1000$ \# maximum number of self-consistent steps

NELMIN $=4 \#$ minimum number of self-consistent steps

$\mathrm{EDIFF}=1 \mathrm{E}-7$ \# convergence criterion for scf-cycle

LWAVE $=$.FALSE.\# do not write out WAVECAR file

LCHARG $=$.FALSE. \# do not write out CHGCAR file

LREAL = Auto \# projection operators in real space

Fig. A.1: INCAR-file for an AIMD simulation for a CO molecule in vacuum.

K-Points \# comment line

1 \# number of $\mathrm{K}$-Points if 0 automatic generation of $\mathrm{k}$-points rec \# $\mathrm{k}$-points in reciprocal coordinates

00001 ! K-point at 0,0,0 with a weight of 1

Fig. A.2: KPOINTS-file for an AIMD simulation for a CO molecule in vacuum. 
$\mathrm{Au}(111)$ vaccum= 20.0 Ang layer: 4 latticeconstant: 4.200 \# comment line 1.00000 \# scaling factor

8.909545900 .000000000 .00000000 \# 1 . vector of simulation cell

-4.454772957 .715892790 .00000000 \# 2. vector\# $\mathrm{p}(3 \mathrm{x} 3)$ slab with 4 layers

0.000000000 .0000000027 .2746143 \# 0 . vector

$1 \quad 1$ \# two species ( $C$ and 0 ) order of POTCAR file

Selective dynamics \# use selective dynamics

Cartesian \# positions in Cartesian coordinates

$5.881723 .0670712 .09948 \mathrm{~T} \mathrm{~T} \mathrm{~T} \mathrm{\#} \mathrm{all} \mathrm{coordinates} \mathrm{allowed} \mathrm{to} \mathrm{move}$

$6.769822 .8383011 .05038 \mathrm{~T} \mathrm{~T} \mathrm{~T} \mathrm{\#} \mathrm{all} \mathrm{coordinates} \mathrm{allowed} \mathrm{to} \mathrm{move}$

Cartesian \# velocities in Ang/fs

$0.040169074-0.010347502-0.073706150$

$\begin{array}{lll}-0.030126806 & 0.007760626 & 0.009333239\end{array}$

Fig. A.3: POSCAR-file for an AIMD simulation for a CO molecule in vacuum.

System Au(111)-slab-NO \# comment line

SYSTEM = Au(111)-slab-NO \# string to describe the system

$\mathrm{GGA}=\mathrm{RP} \# \mathrm{RPBE}$ functional

GGA_COMPAT $=$. FALSE. \# restrores symmetry, recommend for GGA

ISYM $=0 \#$ no symmetry considered

IBRION $=-1$ \# static calculation

ISPIN $=2 \#$ spin-polarized calculations

MAGMOM= $36 * 1.01 * 3.01 *-2.0$ \# initial moment of the species $\mathrm{Au}, \mathrm{N}, 0$

ENCUT $=400 \#$ cut of energy for plane waves

ISMEAR $=1 \#$ MP1 smearing function

SIGMA $=0.2 \#$ width of the smearing function

ALGO $=$ Normal \# algorithm of scf-cycle

PREC = ACCURATE \# precision of calculation

LREAL= Auto \# projection operators in real space

NELM $=200 \#$ maximum number of self-consistent steps

NELMDL $=-20 \#$ number of non-self-consistent steps

$E D I F F=1 E-5 \#$ convergence criterion for scf-cycle

LAECHG $=$.TRUE. \# write out all-electron charge density

LCHARG $=$.TRUE. \# write out CHGCAR file

LDIPOL $=$.TRUE. \# dipole correction

IDIPOL $=3 \#$ dipole corrections in $z$-direction

Fig. A.4: INCAR-file for a NO/Au(111) calculation. 
K-Points \# comment line

0 \# number of $\mathrm{K}$-Points if 0 automatic generation of $\mathrm{k}$-points Gamma \# Generation including the Gamma point

441 \# $\mathrm{k}$-points in $\mathrm{x}^{-}, \mathrm{y}^{-}$, and $\mathrm{z}$-directions

000 \# no shift in all three directions

Fig. A.5: KPOINTS-file for for a NO/Au(111) calculation. 
$\mathrm{Au}(111)$ vaccum $=20.0$ Ang layer: 4 latticeconstant: 4.200

1.00000 \# scaling factor
8.90954590
0.000
$0.000 \# 1$. vector of sim. box
$-4.45477295 \quad 7.71589279$
$0.000 \# 2$. vector of sim. box

$0.000 .00027 .2746143 \# 3$. vector of sim. box 36

1

1 \# number of atoms of each species

Selective dynamics \# use selective dynamics

Cartesian \# positions in Cartesian coordinates

$0.00000000 \quad 0.000000000 .00000000$ F F F

$2.969848630 .00000000 \quad 0.00000000$ F F F

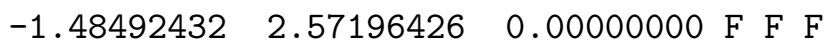

1.484924322 .571964260 .00000000 F F F

$\begin{array}{lll}5.93969727 & 0.00000000 & 0.00000000 \text { F F F }\end{array}$

$\begin{array}{llll}-2.96984863 & 5.14392853 & 0.00000000 \text { F F F }\end{array}$

$\begin{array}{lll}4.45477295 & 2.57196426 & 0.00000000 \text { F F F }\end{array}$

$0.00000000 \quad 5.143928530 .00000000$ F F F

2.969848635 .143928530 .00000000 F F F

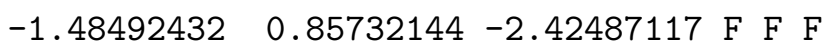

$1.484924320 .85732144-2.42487117$ F F F

$-2.96984863 \quad 3.42928576-2.42487117$ F F F

$0.00000000 \quad 3.42928576-2.42487117$ F F F

$4.45477295 \quad 0.85732144-2.42487117$ F F F

$-4.454772956 .00124979-2.42487117$ F F F

$2.96984863 \quad 3.42928576-2.42487117$ F F F

$-1.484924326 .00124979-2.42487117$ F F F

$1.484924326 .00124979-2.42487117$ F F F

$0.000000001 .71464288-4.84974235$ F F F

$2.969848631 .71464288-4.84974235$ F F F

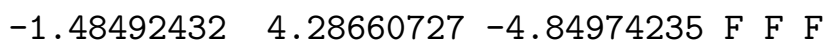

$1.484924324 .28660727-4.84974235$ F F F

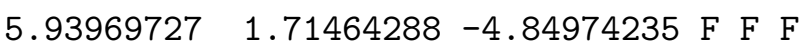

$-2.969848636 .85857153-4.84974235$ F F F

$4.45477295 \quad 4.28660727-4.84974235$ F F F

$0.00000000 \quad 6.85857153-4.84974235$ F F F

$2.969848636 .85857153-4.84974235$ F F F

$0.00000000 \quad 0.00000000-7.27461352$ F F F

$2.969848630 .00000000-7.27461352$ F F F

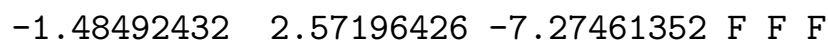

$1.484924322 .57196426-7.27461352$ F F F

$5.939697270 .00000000-7.27461352$ F F F

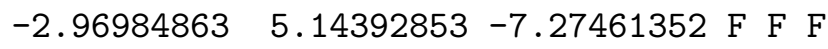

$4.45477295 \quad 2.57196426-7.27461352$ F F F

$0.000000005 .14392853-7.27461352$ F F F

$2.969848635 .14392853-7.27461352$ F F F

$0.00000000 \quad 0.000000007 .386871044$ F F F

0.000000000 .000000008 .536871042 F F F

Fig. A.6: POSCAR-file for a NO/Au(111) calculation. 


\section{A.2 Example input files for FHI-aims}

The examples for FHI-aims consists of a trajectory for $\mathrm{CO}(v=17)$ scattered from a $\mathrm{p}(3 \times 3)$ Ag slab with 4 layers and the input files for the MEP calculation of the CO oxidation on $\operatorname{Pt}(111)$.

\section{A.2.1 Input files for an AIMD simulation of $\mathrm{CO}(v=17) / \operatorname{Ag}(111)$}

In the Figs. A.7 and A.10 the control . in-file (without basis set settings) and the geometry . in-file for an exemplary trajectory of $\mathrm{CO}(\nu=17)$ scattered from $\operatorname{Ag}(111)$ at $300 \mathrm{~K}$ are shown. As examples for the used basis set settings, the light settings for $\mathrm{C}$ and $\mathrm{O}$ are presented in the Figs. A.8 and A.9. The input files were used for the simulations in section 8.4.

\section{A.2.2 Input files for a MEP calculation}

Here, the input files for the MEP calculation of CO on $\mathrm{Pt}(111)$ are presented. This includes the chain. in-, the control. in-file (without basis set settings) in Figs. A.11 and A.12, and the configuration files for the initial (ini.in) and final states ( $\mathrm{f}$ in. in) in the Figs. A.13 and A.14. The calculations, for which these input files are used, are described in section 6.4. 


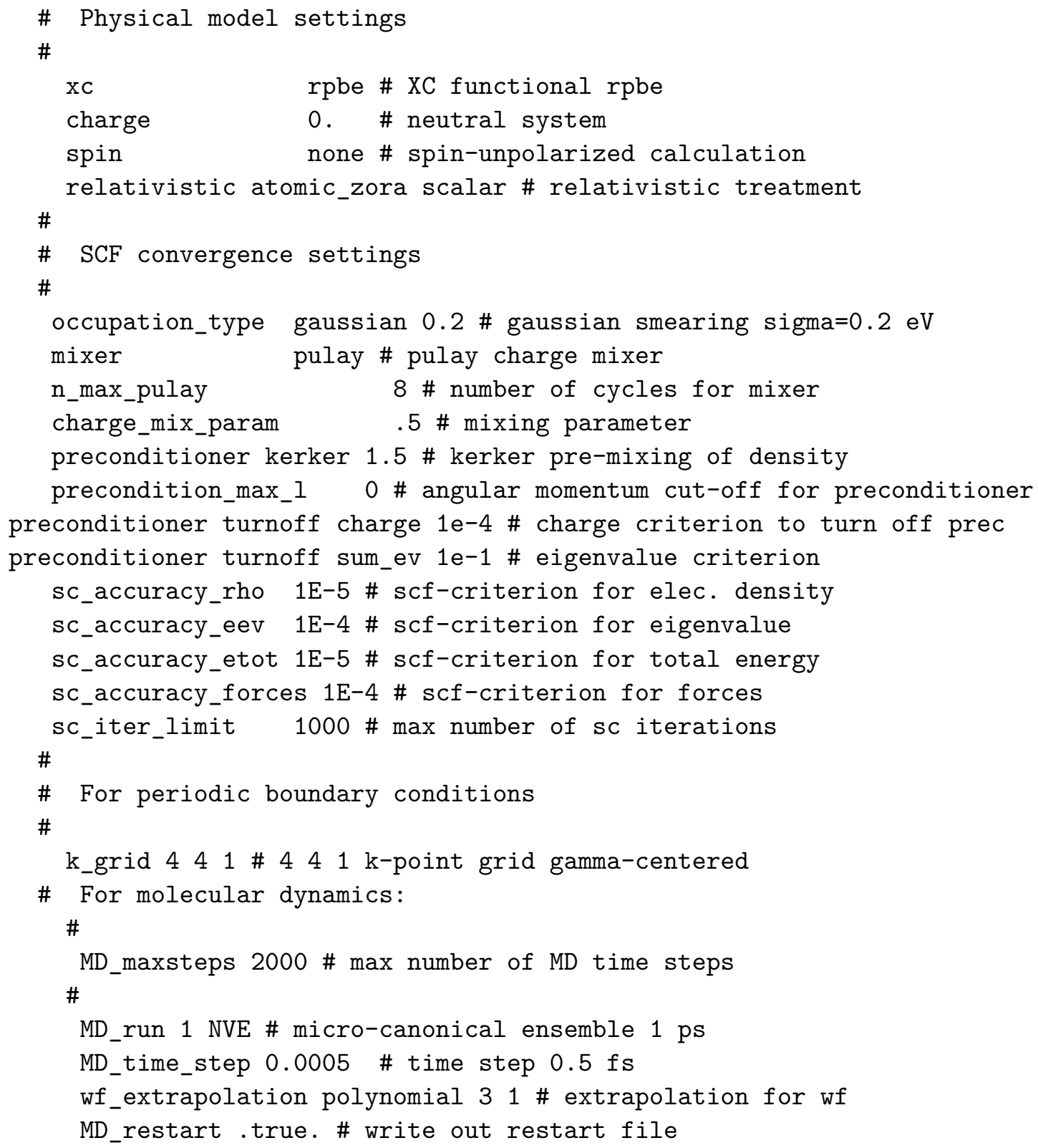

Fig. A.7: control . in-file without basis set settings as used for exemplary AIMD simulations of $\operatorname{CO}(v=$ 17)/Ag(111). 


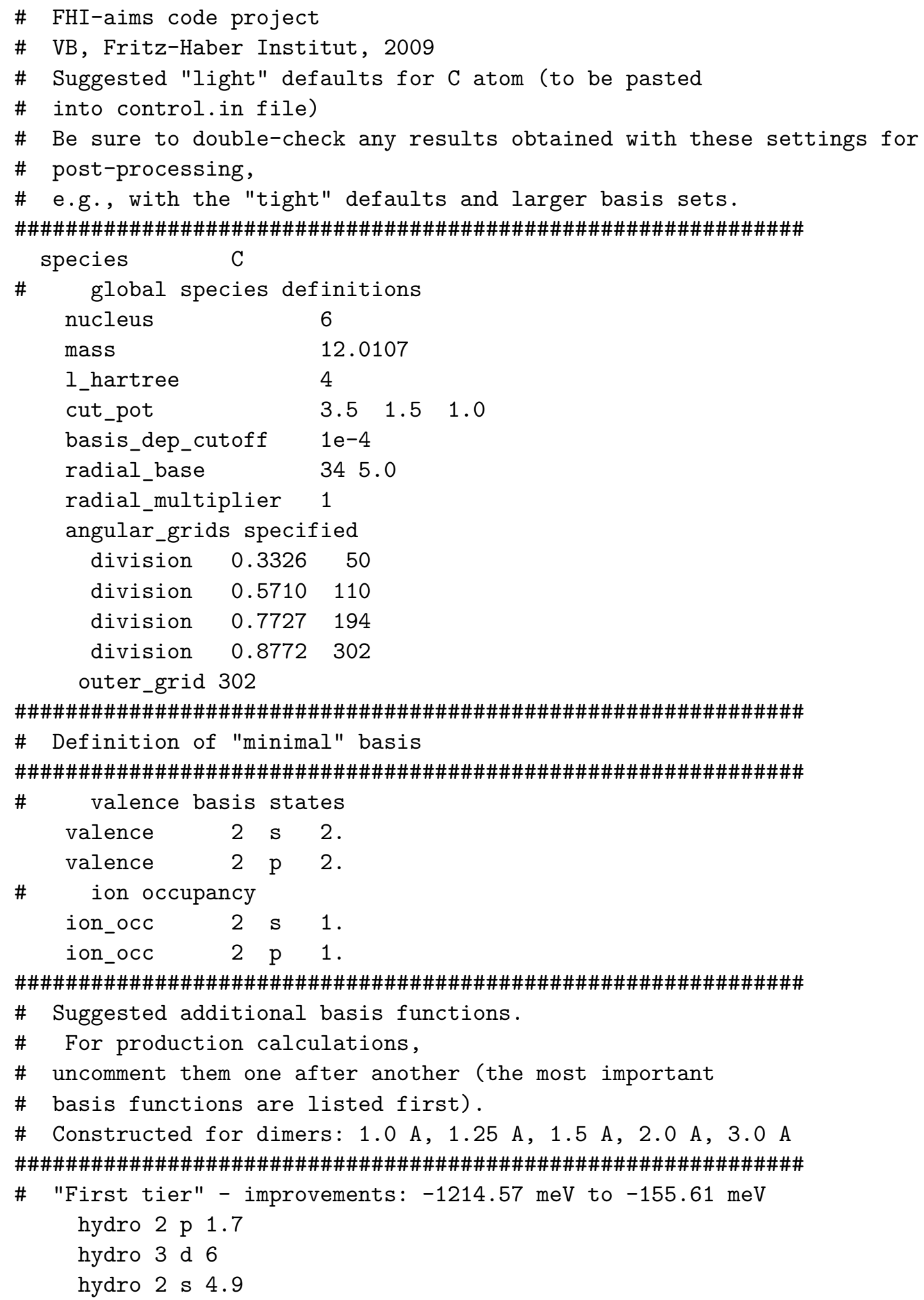

Fig. A.8: Basis set settings (light) for the $\mathrm{C}$ atom. 


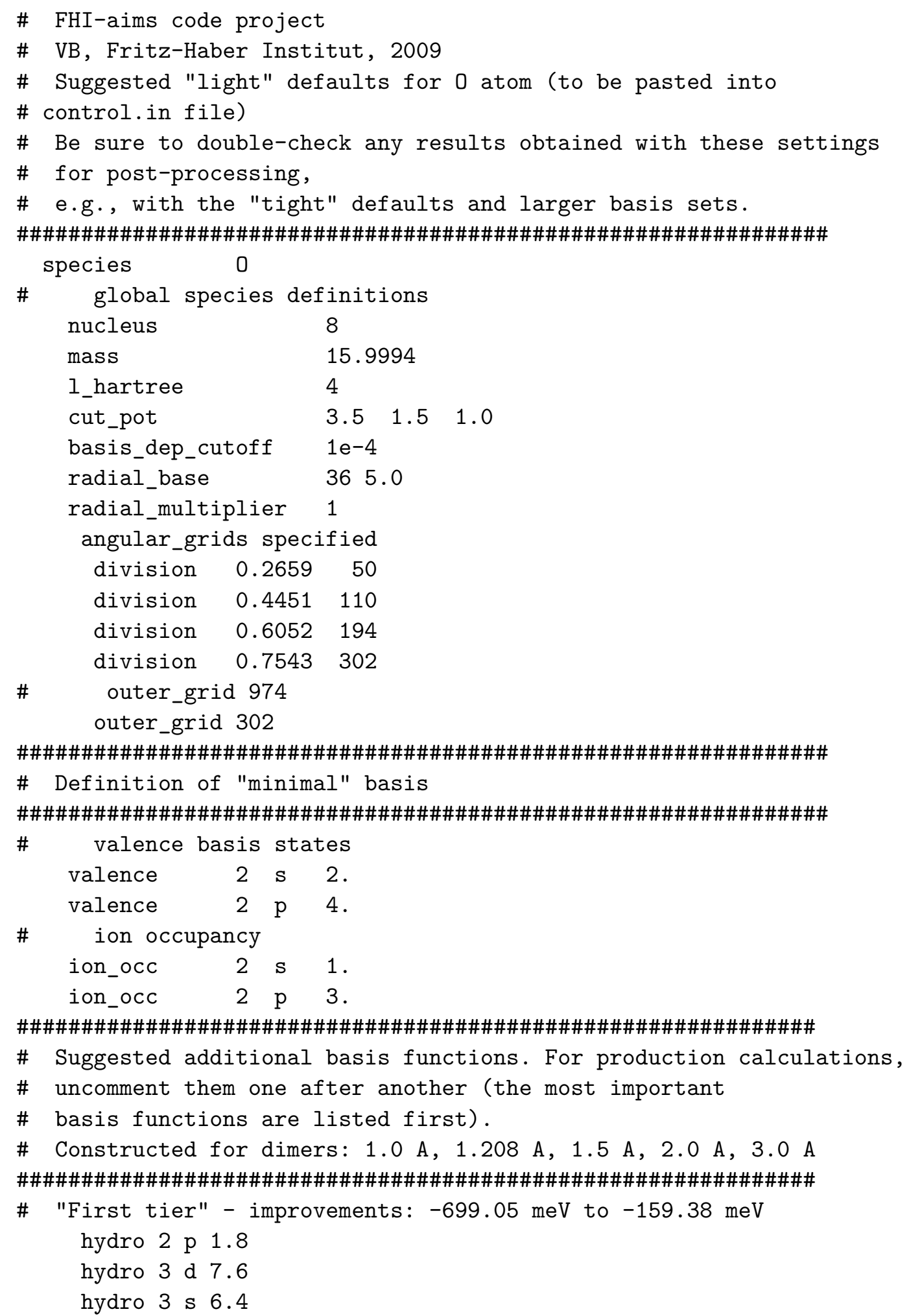

Fig. A.9: Basis set settings (light) for the $\mathrm{O}$ atom. 


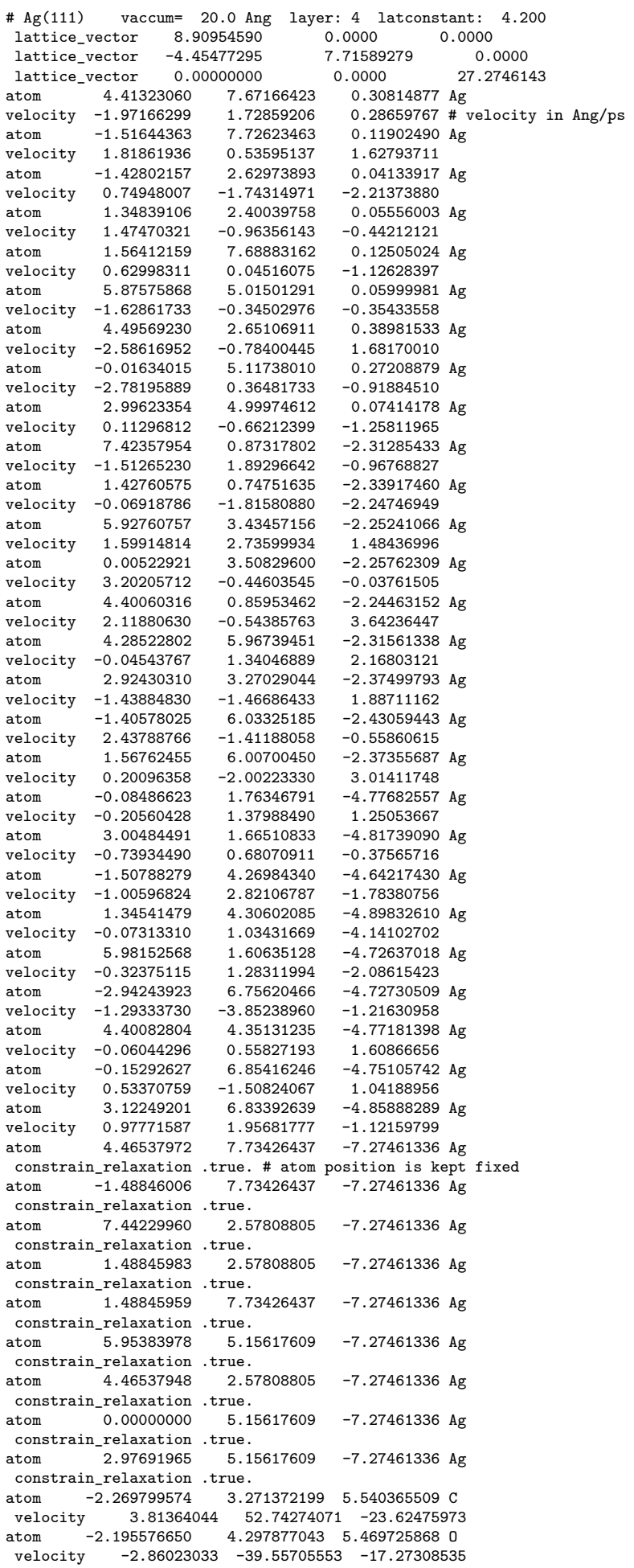

Fig. A.10: geometry . in-file of an exemplary trajectory for $\operatorname{CO}(v=17)$ scattered from $\operatorname{Ag}(111)$. 
run_aims poe aims.160527.scalapack.mpi.x \# aims executable method string \# method for MEP calculation force_thres 0.15 \# force criterion for the whole path initial_file ini.in \# name of file with initial configuration final_file fin.in \# name of file with final configuration n_images 15 \# number of images use_climb true \# using climbing image approach climb_thres 0.05 \# force criterion on climbing image climb_mode 2 \# image with highest energy \# and two neighboring images are allowed to move global_optimizer true \# all images are optimized as one object interpolated_climb true \# climbing image interpolated from energies periodic_interpolation true \# restart true \# write file to restart calculation xyz_lattice 221 \# write out .xyz-path files with 221 image external_geometry extgeo.lst \# uses external geometry files \# for images resample .true. \# resample the path with new images

Fig. A.1 1: chain . in-file as used in the MEP calculation of the CO oxidation.

\# Physical model settings Xc rpbe \# XC functional rpbe vdw_correction_hirshfeld \# TS vdW-corrections charge 0 . \# neutral system spin none \# spin-unpolarized calculation relativistic atomic_zora scalar \# relativistic treatment

\# SCF convergence settings

occupation_type gaussian 0.2 \# gaussian smearing sigma=0.2 eV mixer pulay \# pulay charge mixer

n_max_pulay 10 \# number of cycles for mixer

charge_mix_param $\quad 0.2$ \# mixing parameter

preconditioner kerker 1.5 \# kerker pre-mixing of density

sc_accuracy_rho 1E-5 \# scf-criterion for elec. density

sc_accuracy_eev 1E-5 \# scf-criterion for eigenvalue

sc_accuracy_etot 1E-6 \# scf-criterion for total energy

sc_accuracy_forces 1E-4 \# scf-criterion for forces

sc_iter_limit 1000 \# max number of sc iterations

\# For periodic boundary conditions

k_grid 441 \# 441 k-point grid gamma-centered

Fig. A.12: control. in-file without basis set settings as used in the MEP calculation of the CO oxidation. 
lattice_vector $\quad 8.485280990 .00000000 \quad 0.00000000$ $\begin{array}{lllll}\text { lattice_vector } & -4.24264050 & 7.34846973 & 0.00000000\end{array}$ lattice_vector $0.000000000 \quad 0.0000000026 .92820360$ $\begin{array}{llll}\text { atom } \quad 0.00000000 & 0.00000000 & -0.01997383 \mathrm{Pt}\end{array}$ constrain_relaration

$\begin{array}{lllll}\text { atom } & 2.82842708 & 0.00000000 & -0.01997213 \mathrm{Pt}\end{array}$ constrain relaxation

atom $-1.41421354 \quad 2.44948983-0.01997518 \mathrm{Pt}$ constrain relaxation .true.

atom $1.414213542 .44948983-0.01996712 \mathrm{Pt}$ constrain_relaxation .true.

$\begin{array}{llll}\text { atom } 5.65685415 & 0.00000000 & -0.01998073 & \mathrm{Pt}\end{array}$

constrain_relaxation .true

atom $-2.82842708 \quad 4.89897966-0.01997500 \mathrm{Pt}$ constrain_relaxation .true.

atom 4.24264061 2.44948983 $-0.01997865 \mathrm{Pt}$ constrain_relaxation .true.

atom $\quad 0.00000000 \quad 4.89897966-0.01997625 \mathrm{Pt}$

constrain relaxation true.

$\begin{array}{llll}\text { atom } & 2.82842708 & 4.89897966 & -0.01998187 \mathrm{Pt}\end{array}$

constrain relaxation true.

$\begin{array}{llll}\text { atom } & -1.41421354 & 0.81649661 & -2.33415847 \\ \mathrm{Pt}\end{array}$

constrain relaxation true.

$\begin{array}{llll}\text { atom } & 1.41421354 & 0.81649661 & -2.33415448 \mathrm{Pt}\end{array}$

constrain_relaxation

atom $-2.82842708 \quad 3.26598644 \quad-2.33415886 \mathrm{Pt}$

constrain_relaxation .true

atom $-0.00000000 \quad 3.26598644-2.33415216 \mathrm{Pt}$

constrain_relaxation .true.

$\begin{array}{llll}\text { atom } & 4.24264050 & 0.81649661 & -2.33415797 \\ \mathrm{Pt}\end{array}$

constrain relaxation .true.

atom $\quad-4.24264050 \quad 5.71547604 \quad-2.33415464 \mathrm{Pt}$

constrain_relaxation , true.

atom $2.82842684 \quad 3.26598644 \quad-2.33415512 \mathrm{Pt}$

constrain relaxation true.

atom $-1.41421354 \quad 5.71547604 \quad-2.33415159 \mathrm{Pt}$

constrain relaxation true.

$\begin{array}{llll}\text { atom } 1.41421342 & 5.71547604 & -2.33415743 & \mathrm{Pt}\end{array}$

constrain relaxation true.

atom $-0.00000000 \quad 1.63299322 \quad-4.61108117 \mathrm{Pt}$

constrain

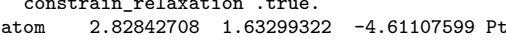

constrain_relaxation .true.

atom -1.41421354 4.08248329 $-4.61107919 \mathrm{Pt}$

constrain_relaxation .true.

atom $1.41421354 \quad 4.08248329-4.61108434 \mathrm{Pt}$

constrain relaxation true.

atom $\quad 5.65685415 \quad 1.63299322 \quad-4.61108091 \mathrm{Pt}$

constrain_relaxation .true.

atom $-2.82842708 \quad 6.53197289-4.61108470 \mathrm{Pt}$

constrain relaxation true.

$\begin{array}{llll}\text { atom } & 4.24264050 & 4.08248329 & -4.61108240 \mathrm{Pt}\end{array}$

constrain

$\begin{array}{llll}\text { atom } 0.00000000 & 6.53197289 & -4.61108391 \mathrm{Pt}\end{array}$

constrai

atom 2.82842708 6.53197289 $-4.61108161 \mathrm{Pt}$

constrain

atom $0.00000000 \quad 0.00000000 \quad-6.92820335 \mathrm{Pt}$

cons

$\begin{array}{lll} & \\ \text { atom } 2.82842708 & 0.00000000 & -6.92820335 \\ \mathrm{Pt}\end{array}$

constrain_relaxation .true.

tom $-1.414213542 .44948983 \quad-6.92820335 \mathrm{Pt}$

constrain_relaxation .true

atom $1.41421354 \quad 2.44948983 \quad-6.92820335 \mathrm{Pt}$

constrain relaxation true.

$\begin{array}{llll}\text { atom } \quad 5.65685415 & 0.00000000 & -6.92820335 \mathrm{Pt}\end{array}$

constrain_relaxation true.

atom $-2.82842708 \quad 4.89897966 \quad-6.92820335 \mathrm{Pt}$

constrain relaxation true.

$\begin{array}{llll}\text { atom } 4.24264061 & 2.44948983 & -6.92820335 & \mathrm{Pt}\end{array}$

constrain relaxation true.

atom $0.00000000 \quad 4.89897966-6.92820335 \mathrm{Pt}$

constrain

constrain_relaxation . true.

constrain_relaxation .true.

$\begin{array}{lllll}\text { atom } & 2.82466485 & 4.90122071 & 2.99664205 & 0 \\ \text { atom } & 2.82580152 & 4.90054258 & 1.84328224 & \mathrm{C}\end{array}$ lattice_vector $8.48528099 \quad 0.00000000 \quad 0.00000000$ lattice_vector $-4.24264050 \quad 7.34846973 \quad 0.00000000$ lattice vector $0.00000000 \quad 0.00000000 \quad 26.92820360$ atom $0.00000000 \quad 0.00000000-0.01997383 \mathrm{Pt}$ constrain_relaxation true.

$\begin{array}{cccc}\text { constrain_relaxation } & \text {.true. } \\ \text { atom } 2.82842708 & 0.00000000 & -0.01997213 \mathrm{Pt}\end{array}$ atom 2.828

$\begin{array}{lll}\text { atom }-1.41421354 & 2.44948983 & -0.01997518 \mathrm{Pt}\end{array}$

$\begin{array}{ccc}\text { constrain_relaxation } & \text { true. } \\ \text { atom } 1.41421354 & 2.44948983 & -0.01996712 \mathrm{Pt}\end{array}$ constrain_relaxation .true.

atom 5.65685415 $0.00000000-0.01998073 \mathrm{Pt}$

constrain_relaxation .true.

atom $\quad-2.82842708 \quad 4.89897966-0.01997500 \mathrm{Pt}$

constrain_relaxation .true.

$\begin{array}{llll}\text { atom } 4.24264061 & 2.44948983 & -0.01997865 \mathrm{Pt}\end{array}$

constrain relaxation .true.

atom $0.00000000 \quad 4.89897966-0.01997625 \mathrm{Pt}$

constrain_relaxation .true.

$\begin{array}{llll}\text { constrain_relaxation } & \text {.true. } \\ \text { atom } 2.82842708 & 4.89897966 & -0.01998187 \mathrm{Pt}\end{array}$

constrain_relaxation .true.

$\begin{array}{llll}\text { atom } & -1.41421354 & 0.81649661 & -2.33415847 \mathrm{Pt}\end{array}$

constrain relaxation true.

$\begin{array}{llll} & \\ \text { atom } 1.41421354 & 0.81649661 & -2.33415448 \mathrm{Pt}\end{array}$

constrain_relaxation, true.

$\begin{array}{llll}\text { atom }-2.82842708 & 3.26598644 & -2.33415886 \mathrm{Pt}\end{array}$

constrain_relaxation .true.

$\begin{array}{llll}\text { atom } 0.00000000 & 3.26598644 & -2.33415216 \mathrm{Pt}\end{array}$

constrain_relaxation true.

$\begin{array}{llll}\text { atom } 4.24264050 & 0.81649661 & -2.33415797 \mathrm{Pt}\end{array}$

constrain_relaxation true.

$\begin{array}{lll}\text { atom } \quad-4.24264050 & 5.71547604 & -2.33415464 \mathrm{Pt}\end{array}$

constrain_relaxation, true.

$\begin{array}{llll}\text { atom } 2.82842684 & 3.26598644 & -2.33415512 \mathrm{Pt}\end{array}$

constrain_relaxation true.

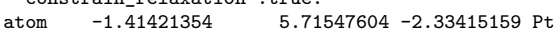

constrain_relaxation .true.

$\begin{array}{llll}\text { atom } 1.41421342 & 5.71547604 & -2.33415743 \mathrm{Pt}\end{array}$

constrain relaxation true.

atom $0.00000000 \quad 1.63299322-4.61108117 \mathrm{Pt}$

constrain_relaxation true.

$\begin{array}{llll}\text { constrain_relaxation } & \text {.true. } \\ \text { atom } 2.82842708 & 1.63299322 & -4.61107599 \mathrm{Pt}\end{array}$

constrain_relaxation .true.

atom $-1.41421354 \quad 4.08248329-4.61107919 \mathrm{Pt}$

constrain_relaxation .true.

$\begin{array}{lll}\text { atom } 1.41421354 & 4.08248329 & -4.61108434 \mathrm{Pt}\end{array}$

constrain_relaxation .true.

$\begin{array}{lll}\text { atom } 5.65685415 & 1.63299322 & -4.61108091 \mathrm{Pt}\end{array}$

constrain_relaxation .true.

$\begin{array}{llll}\text { atom } & -2.82842708 & 6.53197289 & -4.61108470 \mathrm{Pt}\end{array}$

constrain_relaxation true.

$\begin{array}{lll}\text { atom } 4.24264050 & 4.08248329 & -4.61108240 \mathrm{Pt}\end{array}$

constrain_relaxation .true.

$\begin{array}{lll}\text { atom } \quad 0.00000000 & 6.53197289 & -4.61108391 \mathrm{Pt}\end{array}$

constrain_relaxation true.

$\begin{array}{lll} & \\ \text { atom } 2.82842708 & 6.53197289 & -4.61108161 \mathrm{Pt}\end{array}$

constrain relaxation true.

$\begin{array}{llll} & \\ \text { atom } 0.00000000 & 0.00000000 & -6.92820335 & \mathrm{Pt}\end{array}$

constrain relaxation true.

$\begin{array}{llll} & \\ \text { atomstrain_relaxation } & \text {.true. } & & \\ \text { atom } & .82842708 & 0.00000000 & -6.92820335 \mathrm{Pt}\end{array}$

constrain_relaxation .true.

$\begin{array}{lll}\text { atom }-1.41421354 & 2.44948983 & -6.92820335 \mathrm{Pt}\end{array}$

constrain_relaxation .true.

$\begin{array}{llll}\text { atom } 1.41421354 & 2.44948983 & -6.92820335 \mathrm{Pt}\end{array}$

constrain_relaxation true.

$\begin{array}{lll}\text { atom } 5.65685415 & 0.00000000 & -6.92820335 \mathrm{Pt}\end{array}$

constrain_relaxation true.

$\begin{array}{llll}\text { atom }-2.82842708 & 4.89897966 & -6.92820335 \mathrm{Pt}\end{array}$

constrain_relaxation .true.

$\begin{array}{llll}\text { atomstrain_relaxation } & \text { true. } \\ \text { atom } 4.24264061 & 2.44948983 & -6.92820335 \mathrm{Pt}\end{array}$

constrain

atom $0.00000000 \quad 4.89897966-6.92820335 \mathrm{Pt}$

constrain relaxation

$\begin{array}{lll} & \\ \text { atom } 2.82842708 & 4.89897966 & -6.92820335 \mathrm{Pt}\end{array}$

constrain_relaxation .true.

atom $0.03734868 \quad 0.65039830 \quad 3.736264410$

atom $\quad 2.30295553 \quad 1.29722286 \quad 3.702839050$

$\begin{array}{llll}\text { atom } & 1.17012918 & 0.97408640 & 3.71884794 \mathrm{C}\end{array}$

Fig. A.13: ini. in-file for the CO oxidation on a Fig. A.14: $f$ in. in-file for the CO oxidation on a p(3 $\times 3)$ slab with 4 layers. $p(3 \times 3)$ slab with 4 layers. 


\section{A.3 geometry . in-files for $\mathrm{CO}$ on $\mathrm{NaCl}(100)$}

In this section the geometry . in-files of the two different slabs, to represent the $\mathrm{NaCl}(100)$ surface to calculate the transition dipole moment, are shown in Fig. A.15. Both files show the case of a 1 ML CO coverage, i.e. with two CO molecules above the slabs.

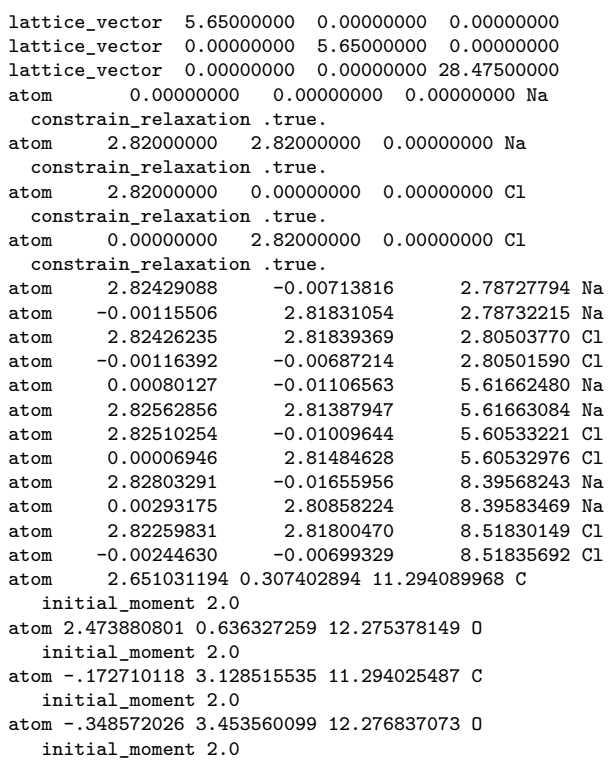

(a) geometry . in-file of the $c(2 \times 1)$ slab with 4 layers within two CO molecules above a $\mathrm{Na}$ atom.

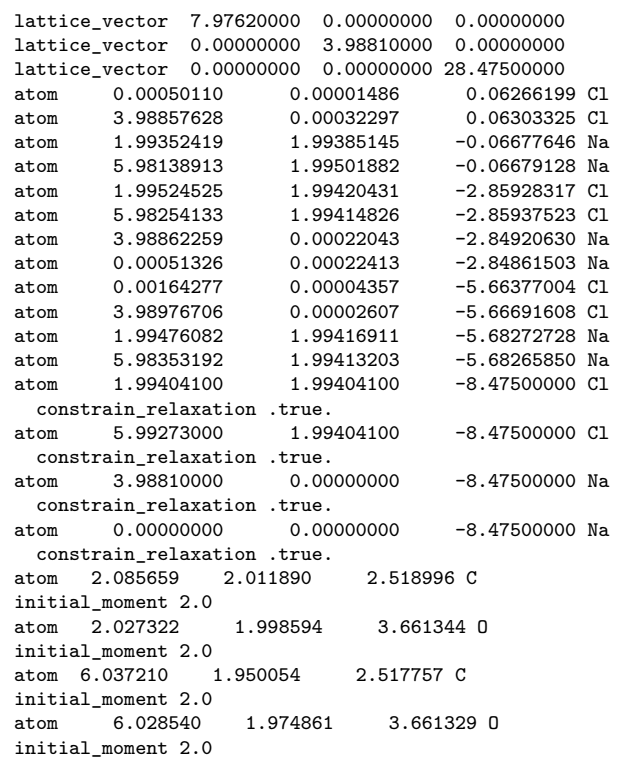

(b) geometry . in-file of the, by $45^{\circ}$ rotated, $\mathrm{c}(2 \times 1) \mathrm{NaCl}(100)$ slab with 4 layers within two $\mathrm{CO}$ molecules above a $\mathrm{Na}$ atom.respectively.

Fig. A.15: geometry . in-files of the used $\mathrm{c}(2 \times 1) \mathrm{NaCl}(100)$ slab with 4 layers (a) and this slab rotated by $45^{\circ}$ around the $z$-axis (b). 